\title{
Search for Sterile Neutrino at MINOS using Antineutrino Disappearance
}

\author{
Thesis submitted to \\ Cochin University of Science and Technology \\ in partial fulfillment of the requirements \\ for the award of the degree of \\ DOCTOR OF PHILOSOPHY \\ by \\ Navaneeth Poonthottathil \\ Department of Physics \\ Cochin University of Science and Technology \\ Kochi - 682022
}

July 2016 
Search for Sterile Neutrino at MINOS using Antineutrino Disappearance

$\mathrm{Ph}$. D. thesis in the field of High Energy Physics

Author

Navaneeth Poonthottathil

Department of Physics

Cochin University of Science and Technology

Kochi - 682022

nav@fnal.gov, navaneethphysics@gmail.com

Research Supervisor

Dr. Ramesh Babu T

Department of Physics

Cochin University of Science and Technology

Kochi - 682022

rameshbabut@gmail.com

Front cover : MINOS Official Logo 
"A billion neutrinos go swimming, one gets wet"

-Michael Kamakana 



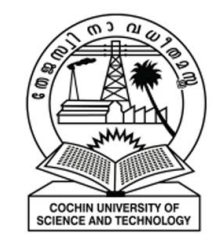

\section{CERTIFICATE}

Certified that the work presented in this thesis entitled "Search for Sterile Neutrino at MINOS using Antineutrino Disappearance" is based on the bonafide record of research work done by Navaneeth P., under our guidance in the Department of Physics, Cochin University of Science and Technology, Cochin 682022 and at Fermi National Accelerator Laboratory, Batavia, IL, USA, 60510 in partial fulfillment of the requirements for the award of degree of Doctor of Philosophy and has not been included in any other thesis submitted for the award of any degree. All the relevant corrections and modifications suggested by the audience during the pre-synopsis seminar and recommendations by doctoral committee of the candidate have been incorporated in the thesis.

Kochi

July, 2016

Batavia, IL

July, 2016
Dr. Ramesh Babu T

(Supervising Guide)
Dr. Robert K Plunkett

(Joint Guide) 



\section{DECLARATION}

I hereby declare that the work presented in this thesis is based on the original research work done by me under the guidance of Dr. Ramesh Babu T, Department of Physics, Cochin University of Science and Technology, Cochin - 682022, India, and Dr. Robert K Plunkett, Fermi National Accelerator Laboratory, Batavia, IL, USA, 60510 and has not been included in any other thesis submitted previously for the award of any degree.

Kochi

July, 2016

Navaneeth $\mathrm{P}$ 



\section{Acknowledgements}

This thesis completed with the help and support from many people. First and foremost, I would like to thank my supervisor Dr. Ramesh Babu Thayyullathil, for his guidance and encouragement throughout my Ph.D. years. Thank you for your advice and support over the last five years.

This thesis work would not possible with out the help of Dr. Robert K Plunkett, my co-supervisor at Fermilab. Rob's nice hospitality and support through out my stay at Fermilab greatly helped me.

I am grateful to Dr. Brajesh Choudhary, he has made all the arrangements to do research work at Fermilab. He has taken care of me as his own student and helped me whenever I asked for.

I am thankful to the conveners of the our group Justin Evans, Joao A. B. Coelho, Mike Kordosky, Alex Sousa, Adam Aurisano, Leigh Whitehead, Jeff de Jong and Ruth Toner who offered me all the help I needed for learning the MINOS software and performing my analysis tasks. I would like to thank Richa Sharma for teaching me the basics of performing an analysis and help me debug my programming mistakes. I would particularly like to thank Benton Pahlka, Son Cao, Rashid Mehdeiyev for helping with software tools when I started work at MINOS. Thanks also to Robert Hatcher and Art Kreymer who were always there to help me with the computing needs.

Thanks to Young MINOS, especially to Alex Radovic, Ashely, Paul, Junting, Simon, Will, Schrkenberger, Michelle, Tom for their support and fun time together. I enjoyed the company with Joe O'Conner during the time he spent at Fermilab.

I remember Dr. Rajendran Raja for making this collaboration possible.

Thanks to the India-Fermilab Neutrino Collaboration, for providing me the opportunity to work at Fermilab and supporting my stay.

Special thanks to Dr. M.K Jayaraj and my MSc co-ordinator Dr. S Jayalekshmi.

I express my special thanks for Head of the Department and all former Heads of the Department of Physics, CUSAT.

Thanks to all teachers and non-teaching staffs.

I would like to thank all my friends at Fermilab. Especially for joining me and made my dinner times more enjoyable.

Thanks to my senior fellow research, junior scholars and MSc friends for the times that we spent together and making my life at CUSAT more 
enjoyable at the time of writing of this thesis, especially to Tharanath, Kunjan, Santhosh, Deepu, Abhilash, Shijeesh, Sajan, Anand, Anshad, Manoj, Jubeesh, Titu, Aravind, Jishnu, Sreeram, Sreejith, Satheesan, Sagar, Dinto, Kurias, Abhay and Manu.

Thanks to my fellow labmates Ashefas, Rijeesh, Sijith and Jubna I J and my senior Priyeshettan for their support.

Finally, this would not have been possible without the support of my family. Many thanks for family for their encouragement. This would not have been possible without you.

Navaneeth $\mathrm{P}$ 


\section{Preface}

Experimental Neutrino Physics is an active area of research in high energy physics. The investigation of the properties of this elusive particles has started since it's discovery itself. In the standard model of particle physics, neutrinos are massless, the discovery of neutrino oscillation is the first evidence that demands the extensions of standard model. It is well established the existence of three active neutrinos, $\nu_{e}, \nu_{\mu}$ and $\nu_{\tau}$, but recent experiments like LSND and MiniBooNE has found some anomalies in their data. These experimental data could not be explained using three flavour neutrino oscillation physics, but they could explain the anomaly by adding a fourth type neutrino called sterile neutrinos. The evidences obtained from these experiments are not yet conclusive. Search for sterile neutrino is an extensive research area in the field of neutrino physics. The main work presented in this thesis is a sterile neutrino search at MINOS (Main Injector Neutrino Oscillation Search) experiment at Fermilab, USA.

MINOS is a two detector experiment at Fermilab, which studies the neutrinos produced at Fermilab Main Injector particle accelerator. MINOS Near Detector is situated at $1 \mathrm{~km}$ away from the source and the Far Detector is at Soudan Mine at Minnesota, $735 \mathrm{~km}$ away from the neutrino source. The experiment is built to study neutrino oscillation phenomena in the atmospheric sector and has made world class measurements on neutrino oscillation parameters. The MINOS experiment is also capable of looking for small perturbation in the energy spectra caused by any fourth type of neutrino and can extract the oscillation parameters.

Chapter 1 briefly explains the history of neutrino physics, which includes Pauli's proposal, discovery of neutrinos and physics of weak interaction. It also describes the discovery of three active neutrinos and phenomena of neutrino oscillation, which is the physics behind the solar and atmospheric neutrino problem. This chapter also contains the mathematical formulation for both two and three flavour neutrino oscillations. It then describes the different oscillation experiment which confirmed the neutrino oscillation phenomena in solar, atmospheric and reactor sector and also briefly explains the aim of many future neutrino experiment around the globe.

Chapter 2 describes the motivation for this thesis, which includes different experimental anomalies, which could be explained by adding a 
forth type neutrino. The evidences includes the results from LSND, MiNiBooNE, Gallium anomaly and reactor anomaly etc. The previous analysis on sterile neutrinos and strategy used in MINOS is also briefly explained.

Chapter 3 contains the details of the MINOS experiment and includes the latest results from the experiment. It also discusses the neutrino beam production, the detector technology and the details of the MINOS data taking, how MINOS take data in neutrino mode and antineutrino mode. The purpose of a two-detector experiment and identifying the different types of events are also briefly described. This chapter also mentions about the MINOS+ experiment, which is a extension of MINOS experiment using higher energy neutrino beam.

Chapter 4 describes the algorithms used for the reconstruction and how we identify and categorise different events. Reconstruction is done in various steps, which is also detailed here. The information about our Monte Carlo simulation chain which is used for the experiment are also included. A brief description of MINOS detector calibration (a multi-stage calibration procedure) is also explained in this chapter.

Chapter $\mathbf{5}$ includes the absolute energy calibration procedure which is the final stage of our detector calibration procedure. The only way to know the detector response over time for a known energy of particle at underground is to exploits the cosmic muons. The technique of how we use the cosmic muons as standard candle for the absolute calibration is explained in this chapter. It also describes the correction applied to this cosmic muons due to their angle dependence at the detector. The response is calculated for both detectors. The chapter also mentions difference in response of muon and anti-muon (charge dependence). The various systematics associated with this study are also investigated. The study of the detector response as a function of the intensity (number of neutrinos) for the beam muons is also described here.

Chapter 6 explains in detail about how we looked for sterile neutrinos at MINOS in the disappearance channel $P\left(\bar{\nu}_{\mu} \rightarrow \bar{\nu}_{\mu}\right)$ in an antineutrino enhanced beam that MINOS collected between 2009-2011. The analysis includes only the charged current events. The charged current event selection for this analysis is detailed. The data corresponds to a Proton -on-Target $(\mathrm{POT})$ of $3.36 \times 10^{20}$ POT. Oscillations to sterile neutrinos can happen due to large range of $\Delta m^{2}$. Hence depending on the baseline neutrino oscillation can happen at both detector. The analysis is done using 
Far/Near $(\mathrm{F} / \mathrm{N})$ fit and look for the perturbation from the standard three flavour oscillation. As we are looking for the small effect, the systematics must be taken so carefully and should be added in the fit. The chapter also describes the various source of systematics and how we included in this analysis. We use covariance matrix technique to add bib-to-bin correlated systematics in the analysis. We have put a limit on the sterile neutrino mixing parameter $\theta_{24}$ over a large range of $\Delta m_{41}^{2}$ at a $90 \%$ confidence limit. The Feldman-Cousins technique for correcting the contours are also described in this chapter. By combining our disappearance limit to BUGEY (a reactor experiment at France) disappearance $P\left(\bar{\nu}_{e} \rightarrow \bar{\nu}_{e}\right)$ limit, we can compare our results to LSND and MiNiBooNE appearance $P\left(\bar{\nu}_{\mu} \rightarrow \bar{\nu}_{e}\right)$ limit.

Chapter 7 summarises the work and the also contains future outlook for sterile neutrino search around the world. The future sensitivity expected for one year of MINOS+ high statistics antineutrino mode is also examined. 


\section{Papers}

\section{MINOS Publications}

- P. Adamson et. al. (MINOS Collaboration), "Measurement of the Multiple Muon Charge Ratio in the MINOS Far Detector", Phys. Rev. D 93, 052017 (2016).

- P. Adamson et. al. (MINOS Collaboration), "The NuMI neutrino beam", Nucl. Instrum. Meth. A806 (2016) 279-306.

- P. Adamson et. al. (MINOS Collaboration), "Search for Sterile antineutrinos in MINOS using Antineutrino Disappearance", Bulletin of the American Physical Society 60 (2015).

- P. Adamson et. al. (MINOS Collaboration), "Observation of seasonal variation of atmospheric multiple muon events in the MINOS Near and Far Detector ", Phys. Rev. D 91, 112006 (2015).

- P. Adamson et. al. (MINOS Collaboration), "Precision measurement of the speed of propagation of neutrinos using the MINOS detectors", Phys.Rev.D 92, 052005 (2015).

- P. Adamson et. al. (MINOS Collaboration), "Study of quasi-elastic scattering using charged-current $\nu_{\mu}$-iron interactions in the MINOS near detector", Phys.Rev. D 91, 012005 (2015).

- P. Adamson et. al. (MINOS Collaboration), "Combined Analysis of $\nu_{\mu}$ disappearance and $\nu_{\mu} \rightarrow \nu_{e}$ appearance in MINOS using Accelerator and Atmospherics neutrinos", Phys. Rev. Lett. 112, 191801 (2014).

\section{MINOS Internal Notes}

- N. Poonthottathil, "A Position Paper for MINOS RHC Sterile Search MINOS ", MINOS DocDB -10928 (2015).

- J.Coelho et.al, "Blessing Package for the 2014 Sterile Analysis", MINOS DocDB -10583 (2014).

- N. Poonthottathil, "Energy resolution systematics Position paper", MINOS DocDB -10571 (2013). 
- N. Poonthottathil, "The MEU number in MINOS+ and the $2 \mathrm{PE}$ issues", MINOS DocDB -10265 (2013).

\section{Conference Talks \& Posters}

- Presented a talk on "Sterile neutrino Search in MINOS using Antineutrinos" at New Perspective meeting, 08th - 09th June 2015, Fermilab, USA.

- Presented a talk on "Sterile neutrino Search in MINOS using Antineutrinos" at New Perspective meeting, 08th - 09th June 2015, Fermilab, USA.

- Presented a talk on "Search for Sterile Neutrinos at MINOS using Antineutrino Disappearance" at American Physical Society meeting, 11th - 14th April 2015, Baltimore, USA.

- Presented a talk on "Selection of Neutral current $\nu_{\mu}$ events in Minos Near detector for Sterile neutrino Search" at American Physical Society meeting, 5th - 8th April 2014, Savannah, USA.

- Presented a talk on "Long Term Performance of the MINOS Calibration Procedure" at New Perspective meeting, 08th - 09th June 2013, Fermilab, USA.

- Presented a talk on "Calibration of MINOS Detectors using Stopping Muons", 7th February 2013, Guwahati, India.

- Presented a poster titled "Search for Muon Antineutrino Disappearance due to Sterile Antineutrino Oscillations with MINOS/MINOS+" at International conference on Neutrino and Astrophysics, Neutrino 2016, South Kensington, London.

- Presented a poster titled "Prospects for Sterile neutrino search in MINOS+" at International conference on Neutrino and Astrophysics, Neutrino 2014, 1st - 7th June 2014, Boston, USA.

- Presented a poster titled "Relative Energy Calibration of MINOS Detectors" at the $46^{\text {th }}$ Fermilab Users' Meeting, 12th - 13th June 2013, Fermilab, USA. 


\section{Conferences Attended}

- Attended $48^{\text {th }}$ Fermilab Annual Users' Meeting, 9th - 11th June 2015, Fermilab, USA.

- Attended American Physical Society Meeting, 11th - 14th April 2015, Baltimore, USA.

- Attended $47^{\text {th }}$ Fermilab Annual Users' Meeting,11th - 12th June 2014, Fermilab, USA.

- Attended an International Conference on Neutrino and Astrophysics, Neutrino 2014, 1st- 7th June 2014, Boston, USA.

- Attended American Physical Society Meeting, 5th - 8th April 2014, Savannah, USA.

- Attended $46^{\text {th }}$ Fermilab Annual Users' Meeting, 12th - 13th June 2013, Fermilab, USA.

- Attended $45^{\text {th }}$ Fermilab Annual Users' Meeting, 12th - 13th June 2012, Fermilab, USA.

- Attended VIII SERC school in Experimental High Energy Physics, 20th June 2011 - 9th July 2011, VECC, Kolkata, India.

- Attended an International Conference on Nanoscience and Nanotechnology, (2011), Cochin University of Science \& Technology (CUSAT), India.

- Attended a National Conference on Photonic Materials, (2008), Cochin University of Science \& Technology (CUSAT), India.

- Attended a workshop on Theoretical Astrophysics, (2008), Cochin University of Science \& Technology (CUSAT), India. 



\section{Contents}

Acknowledgements $\quad$ iii

Preface v v

List of Publication and Conference Attended ix

List of Tables

\begin{tabular}{ll}
\hline List of Figures & 7
\end{tabular}

\begin{tabular}{lll}
\hline & Introduction to Neutrinos & 19
\end{tabular}

1.0 .1 The Weak Interaction . . . . . . . . . . . . . . 19

1.0 .2 Three types of Neutrinos . . . . . . . . . . . . . . . 21

$1.0 .3 \quad$ Neutrino Flavour Change and Mass . . . . . . . . . 22

1.1 Neutrino Oscillations . . . . . . . . . . . . . . . . . 22

1.2 The Two Flavour Approximation . . . . . . . . . . . . . . 26

$\begin{array}{lll}1.3 & \text { Experimental evidence for Neutrino oscillations }\end{array} \ldots$. . . . . 28

1.3.1 Solar Neutrinos . . . . . . . . . . . . . . . . . 28

1.3 .2 Atmospheric Neutrinos . . . . . . . . . . . . . . . 32

1.3.3 Reactor Neutrino Oscillation . . . . . . . . . . . . . 35

1.4 Future Neutrino Programmes . . . . . . . . . . . . . . . . 39

1.4.1 Mass Ordering . . . . . . . . . . . . . . . . . 39

1.4 .2 CP violation . . . . . . . . . . . . . . 40

$1.4 .3 \theta_{23}$ Octant $\ldots \ldots \ldots \ldots \ldots$

2 Sterile Neutrinos 43

$2.1 \quad$ Experimental Anomalies in Neutrino

Physics........................ . . 44

2.1 .1 The LSND Anomaly . . . . . . . . . . . . . . . . 44

2.1 .2 The MiniBooNE Experiment. . . . . . . . . . . . 46

2.1.3 The Short Baseline (SBL) Reactor Anomalies . . . 49

2.1 .4 The Gallium Anomaly . . . . . . . . . . . . . 50

2.2 Four-Flavour Oscillation Model (3+1 Model) . . . . . . . . 51

2.3 Sterile Neutrinos in MINOS . . . . . . . . . . . . . . . 53

2.4 Context of the Thesis . . . . . . . . . . . . . . . . 56 
3 The NuMI Beam and the MINOS Experiment 57

3.1 MINOS Physics . . . . . . . . . . . . . . . . 58

3.2 The NuMI Beam . . . . . . . . . . . . . . . . . . . . . 59

3.2 .1 The Data Collection . . . . . . . . . . . . . 61

3.2 .2 Results of the MINOS Combined Analysis . . . . . 64

3.3 The MINOS Detectors . . . . . . . . . . . . . . . 65

3.3 .1 The Near Detector . . . . . . . . . . . . . 66

3.3 .2 The Far Detector . . . . . . . . . . . . . . . . 67

3.3 .3 The Calibration Detector. . . . . . . . . . . . . . 68

3.3 .4 The Magnetic Field . . . . . . . . . . . . . . . . . . . 68

3.4 Data Acquisition and Trigger System . . . . . . . . . . . . 69

3.5 Interactions in the MINOS Detectors . . . . . . . . . . . . 71

3.5.1 Charged Current $v_{\mu}$ Interactions . . . . . . . . 71

3.5 .2 Neutral Current Interactions . . . . . . . . . . . . . 72

3.5.3 Charged Current $v_{\mathrm{e}}$ Interactions . . . . . . . . . . . 73

3.6 The MINOS+ Experiment . . . . . . . . . . . . . . . . 73

\begin{tabular}{|lll}
4 & Event Reconstruction, MC Simulation and Calibration & 75
\end{tabular}

4.0 .1 Digit Formation . . . . . . . . . . . . . . 75

4.0 .2 Strip Formation and Slicing . . . . . . . . . . . . 76

4.0 .3 Track Reconstruction . . . . . . . . . . . . . . . . 77

4.0 .4 Shower Reconstruction . . . . . . . . . . . . . . . 78

4.0 .5 Formation of Event . . . . . . . . . . . . . . . . . 80

4.1 MC Simulation . . . . . . . . . . . . . . . . . . 80

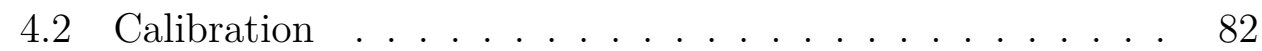

4.2 .1 Drift Calibration . . . . . . . . . . . . . . . . 83

4.2 .2 Linearity . . . . . . . . . . . . . . . . . . . . . . . . 83

$4.2 .3 \quad$ Strip to Strip calibration . . . . . . . . . . . . . . . . 84

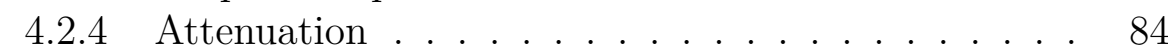

4.2 .5 Relative Energy Calibration . . . . . . . . . . . . 84

4.3 Absolute Energy Scale . . . . . . . . . . . . . . . . 85

\begin{tabular}{|lll}
5 & Absolute Energy Calibration of MINOS Detectors & 89
\end{tabular}

$5.1 \quad$ Relative Energy Calibration _. . . . . . . . . . 89

5.1.1 Calculation of MEU - Track Window Method . . . 90

5.1 .2 Far Detector Performance Over Time . . . . . . . . 92

5.1 .3 Near Detector Performance Over Time . . . . . . . 94 
5.1.4 Angular Dependence on Near and Far Detector . . 96

5.2 MEU Systematics . . . . . . . . . . . . . . . . . 97

5.3 Intensity effect on Beam MEU . . . . . . . . . . . . . . . 98

5.4 Need of Relative Energy Calibration . . . . . . . . . . . . 100

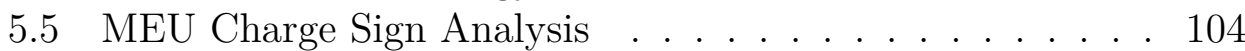

5.6 Summary and Conclusion . . . . . . . . . . . . . . . 106

6 Sterile Neutrino Search using Antineutrinos 109

6.1 Sterile Neutrinos . . . . . . . . . . . . . . . . . . 109

6.2 Sterile Neutrinos in MINOS . . . . . . . . . . . . . . . . . 110

6.3 Data Stability and Preselection of Antineutrino Events . . 112

6.3.1 Near and Far Detector Fiducial Volume Criteria . . 114

6.4 Event Selection Performance . . . . . . . . . . . . . . . . 114

6.5 Previous MINOS Analysis . . . . . . . . . . . . . . . . . 114

6.6 New Analysis Strategy . . . . . . . . . . . . . . . . . . . . 115

6.7 Binning Scheme . . . . . . . . . . . . . . . . . . . . . . . 117

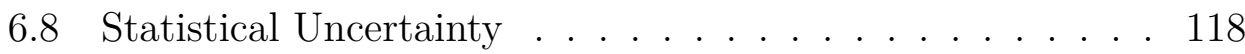

6.9 Systematics Uncertainties . . . . . . . . . . . . . . . . . . 119

6.9 .1 Hadron Production . . . . . . . . . . . . . . . . . . 119

6.9 .2 Normalisation Systematics . . . . . . . . . . . . . 120

6.9 .3 Acceptance Systematics . . . . . . . . . . . . . . . 120

$6.9 .4 \quad$ Beam Optics systematics . . . . . . . . . . . . . . 123

6.9 .5 Cross Section Systematics . . . . . . . . . . . . . . 124

6.9.6 The Statistical and Systematic Error Band on $F / N$

6.10 Sensitivity . . . . . . . . . . . . . . 126

6.11 Sensitivity with Systematics . . . . . . . . . . . . . . 127

6.12 Extended Contour and Surface in $\Delta m_{41}^{2} \ldots \ldots$. . . . . . . 128

6.13 Feldman Cousins . . . . . . . . . . . . . . . . . . . . . 130

$6.14 \quad F / N$ Ratio and Data Results . . . . . . . . . . . . . . . . 132

6.15 Feldman-Cousins Corrected Surface . . . . . . . . . . . . . 134

6.16 Combination with Bugey . . . . . . . . . . . . . . . 136

6.17 Future Antineutrinos in MINOS+ . . . . . . . . . . . . . . 137

6.18 Combination with Bugey in MINOS + . . . . . . . . . . . 139

6.19 Conclusion . . . . . . . . . . . . . . . . . . 139

\begin{tabular}{|lll}
7 & Conclusions and Future Scope & 141
\end{tabular} 


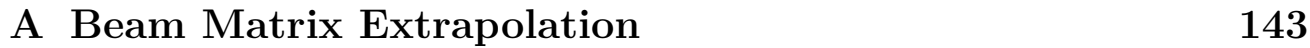

A.1 Extrapolation Method . . . . . . . . . . . . . . . . . . . 143

B Neutral Current Event Selection at Near Detector for An$\begin{array}{ll}\text { tineutrino } & 149\end{array}$

B.1 Introduction . . . . . . . . . . . . . . . . . . . . . . . 149

B.2 Neutral Current Event Selection . . . . . . . . . . . . . . . 152

B.3 Sterile Neutrino Search Strategy Using NC Events . . . . 155

\begin{tabular}{ll}
\hline References & 157
\end{tabular} 


\section{List of Tables}

2.1 Values of $R, N D_{\{\text {data }\}}, S_{\mathrm{NC}}$ and the contributions to $B_{\mathrm{CC}}$ for various reconstructed energy ranges. The numbers in parentheses are calculated including $v_{\mathrm{e}}$ appearance at the the experiment CHOOZ (a place in France) limit. The first uncertainty in the value of $\mathrm{R}$ shown is the statistical uncertainty, the second is systematic uncertainty, and the third is due to possible $v_{\mathrm{e}}$ appearance. Table taken from [71].]. . $\quad 55$

3.1 Summary of the MINOS Run Periods. MINOS collected data mostly in low energy (LE) configuration. The experiment has taken data in pseudo high energy (pHE), where neutrino energy is $7 \mathrm{GeV} . \ldots \ldots \ldots 3$

$5.1 \quad$ MEU systematics table . . . . . . . . . . . . . . . . . . . . . 99

5.2 MEU charge sign study table . . . . . . . . . . . . . . 105

6.1 The wrong sign background and NC contamination . . . . 114

6.2 Three flavor oscillation parameters . . . . . . . . . . 116 



\section{List of Figures}

1.1 Two possible types of weak interaction. The charged current interaction (left) via $W$ boson exchange and neutral current interaction (right) via exchange of $Z^{0}$. This is applicable to both neutrinos and antineutrinos. . . . . . . . . 21

1.2 A history of neutrino physics in brief. Figure is taken from

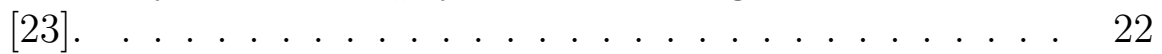

1.3 The predicted solar neutrino flux by SSM. The numbers shown are the uncertainty from the models. . . . . . . . . 29

1.4 The ratio of measured rate of $\bar{v}_{e}$ to the expectation for no oscillation as function of $L / E$. Also shown the best fit for oscillation hypothesis. Figure is taken from [39]. . . . . . . 31

1.5 The neutrino oscillation parameters allowed by KamLAND and combined solar neutrino experiments. The side panels show the $\Delta \chi^{2}$ profiles for KamLAND (dashed line) and solar experiments (dotted line) individually, and the combination of the two (solid line). Figure is taken from [39].]. 32

1.6 Zenith angle distributions of $\mu$-like and e-like events for sub-GeV and multi-GeV data set. The dotted lines are the prediction from the Monte Carlo with out oscillation and the solid line is the best fit to the $v_{\mu} \rightarrow v_{\tau}$ oscillations. The sub-GeV events are with energy $<1.33 \mathrm{GeV}$, mutli-GeV events are with energy $>1.4 \mathrm{GeV}$. Plot is taken from [42] .33

1.7 The $90 \%$ C.L contour for $\Delta m^{2}$ vs $\sin ^{2}(2 \theta)$ from MINOS. This uses the full set of MINOS data which includes $v_{\mu}$ mode, $\bar{v}_{\mu}$ mode and 37.88 kiloton-year atmospheric data. The contour obtained from the Super-K $L / E$ analysis and T2K 90\% contour are shown [43]. . . . . . . . . . 34 
1.8 Top: The prompt energy spectrum of the far hall (sum of three ADs) compared with the no-oscillation prediction based on the measurements of the two near halls. Spectra were background subtracted. Uncertainties are statistical only. Bottom: The ratio of measured and predicted nooscillation spectra. The solid curve is the expected ratio with oscillations, calculated as a function of neutrino energy assuming $\sin ^{2} 2 \theta_{13}=0.089$ obtained from the rate based analysis. The dashed line is the no-oscillation prediction. Plot is taken from [47]. . . . . . . . . . . . . . 36

1.9 The observed prompt energy spectrum at Far Detector compared with the non-oscillation predictions from the measurements in the Near Detector. The backgrounds shown in the inset are subtracted for the far spectrum. The background fraction is $5.5 \%$ (2.7\%) for Far (Near) detector. Errors are statistical uncertainties only. Bottom: The ratio of the measured spectrum of Far Detector to the nonoscillation prediction. Plot is taken from [4].

1.10 The $68 \%$ and $90 \%$ C.L for $\sin ^{2} 2 \theta_{13}$ scanned over values of $\delta_{C P}$. Left plot for normal hierarchy and right plot is inverted hierarchy. Plot is taken from [49].] . . . . . . . . . 38

1.11 The $68 \%$ and $90 \%$ C.L for $\sin ^{2} 2 \theta_{13}$ values of $\delta_{C P}$ for MINOS (left) [50] and the latest results from NOvA, showing the $\sin ^{2} 2 \theta_{13}$ scanned over values of $\delta_{C P}$ for normal mass ordering and inverted mass ordering (right). . . . . . . . . 39

1.12 Two possible mass ordering for neutrino. . . . . . . . . . . 40

2.1 The observed excess in $v_{\mathrm{e}}$ events in LSND as function of energy (left) and as a function of $L / E$ (right). Plots taken

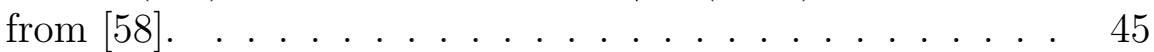

2.2 The fit results in terms of parameters space $\left(\Delta m^{2}\right.$ and $\left.\sin ^{2} 2 \theta\right)$. The fit includes both $\bar{v}_{\mathrm{e}} \rightarrow \bar{v}_{\mu}$ and $v_{\mathrm{e}} \rightarrow v_{\mu}$ oscillations. The $90 \%$ Confidence Limit (90\%C.L) from reactor Bugey experiment (green) and KARMEN2 (brown) are also shown (left). Combined analysis of KARMEN and LSND \begin{tabular}{|l|l|}
\hline gives the allowed region (right). The figure taken from [64]. & 45
\end{tabular} 
2.3 The flux prediction for different neutrino component at the MiniBooNE detector, for neutrino mode (right) and antineutrino mode (left) [64]. $\ldots \ldots \ldots \ldots$. . . . . . . . . 46

2.4 The reconstructed energy spectra for neutrino in MiniBooNE detector with different backgrounds (top). The excess of neutrino events seen in the MiniBooNE as function of $E^{Q E}$ (bottom). Figure taken from [64]. . . . . . . . . . . . . . 47

2.5 The reconstructed energy spectra for antineutrino in MiniBooNE with different backgrounds (right). The excess of antineutrino events seen in the MiniBooNE as function of $E^{Q E}$ (left). Figure taken from 64. . . . . . . . . . . . . 48

2.6 The allowed region of parameter space obtained from the MiniBooNE antineutrino fit result, which is consistent with LSND signal [64].

2.7 The top plot shows the sensitivity (dashed line) and limit (solid line) for 90\% C.L. for neutrino disappearance in MiniBooNE. Previous limits by CCFR (Chicago - Columbia Fermilab - Rochester) in dark grey and CDHS (CERN Dortmund - Heidelberg - Saclay experiment) in light grey are also shown. The bottom plot shows the antineutrino disappearance limit [66]. . . . . . . . . . . . . . . . . 49

2.8 The allowed regions of oscillation parameters from the SBL data with $3+1$ model (left). Ratio of observed to expected number of events, together with prediction for different $\Delta m^{2}$ and $\sin ^{2} 2 \theta_{14}$ values (right). The figure is taken from

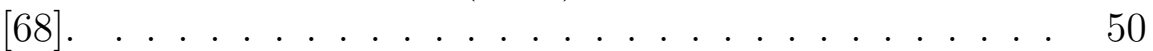

2.9 The allowed region in the $\Delta m^{2}-\sin ^{2} 2 \theta$ plane obtained from the combined fit of the results of the two GALLEX $\mathrm{Cr}$ radioactive source experiments, and the SAGE $\mathrm{Cr}$ and Ar radioactive source experiments [64]. . . . . . . . . . . 51

2.10 The plot shows the reconstructed NC selected events in MINOS Near Detector data and compared with MC simulation, the band shows $1 \sigma$ error in the MC, the CC background is also shown in the back histogram. Figure taken

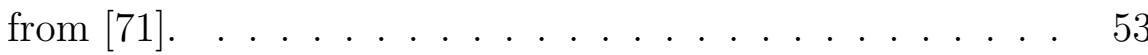


2.11 The plot shows the reconstructed NC selected events in MINOS Far Detector data and compared with MC simulation, the band shows $1 \sigma$ error in the MC, also the CC background is also shown in the back histogram. Figure is taken from [71]. $\ldots \ldots \ldots \ldots \ldots$. . . . . . . . . . . . 54

3.1 Schematic representation of the MINOS experiment. The Near Detector (ND) is at Fermilab and the Far Detector

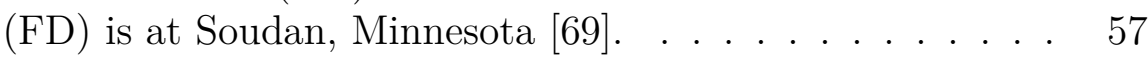

3.2 (a) The results of the MINOS disappearance with $v_{\mu}$ beam data, comparing the FD data (black) to the prediction without oscillation (red) and the prediction with oscillation (blue). Data agrees well with the oscillation prediction. (b) The ratio to the no oscillation, a clear oscillation dip is visible, the best fit oscillation are shown in blue. . . . . . . 58

3.3 The survival probability of a muon neutrino in a two-flavour model as a function of neutrino energy, the position of the dip is the measure of mass squared splitting, $\Delta m_{\mathrm{atm}}^{2}$ and depth of the dip is the measure of mixing angle, $\sin ^{2} 2 \theta$. Figure is taken from [69]. . . . . . . . . . . . . . . . 59

3.4 The graphical representation of the NuMI beam and neutrino production. The figure is taken from [23]. $\ldots$. . . . . 60

3.5 Configurations of the NuMI beam for the different neutrino energy spectrum. The relative positions of horns and target for the 3 different configurations, Low (LE), Medium (ME) and High (HE) Energy [69. . . . . . . . . . . . . . . . 61

3.6 The $v_{\mu}$ and $\bar{v}_{\mu}$ at the Near Detector (a) For $v_{\mu}$ mode running, (b) $\bar{v}_{\mu}$ mode running. . . . . ........ 61

3.7 The total POT of data collect during the time of operation of MINOS between 2005-2012. Green represents $v_{\mu}$ running, orange represents the $\bar{v}_{\mu}$ mode running and red is for pseudo-high energy beam. . . . . . . . . . . . . . 62 
3.8 Multi-panel contour and profile plots from combined fit to

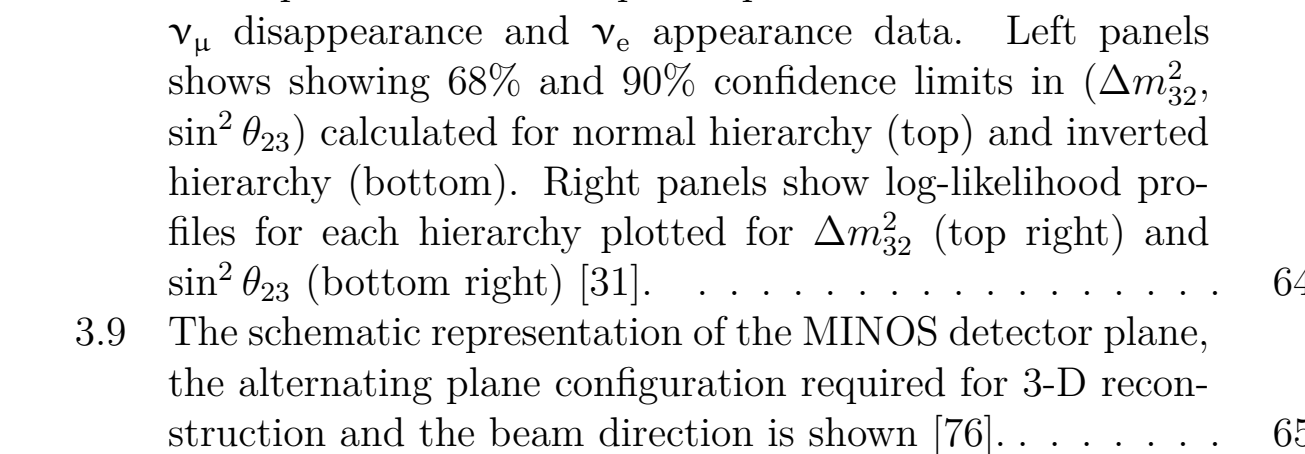

3.10 The detailed view of the MINOS scintillator strip, when MIP (minimum ionising particle) pass through the scintillator photons are produced, which then collected in wavelength shifted cable and transported to the photomultiplier tube at the edge of the detector $[76] . \ldots \ldots . . \ldots 65$

3.11 A typical ND partially instrumented plane. The shaded grey color represents the covered scintillator portion. For a fully instrumented region the plane is fully covered with scintillator plane. The center diamond shows the coil and the dark circle represent the beam center [76]. . . . . . . . 66

3.12 The different configuration of scintillator strip module on the Near Detector plane [76]. . . . . . . . . . . . . . . 67

3.13 Near Detector at Fermilab (left) and Far Detector at Soudan Underground Laboratory at Minnesota (right). . . . . . . . 68

3.14 Near Detector magnetic field map (left) and Far Detector magnetic field map (right). The grey scale strength of the magnetic field is calculated by finite element analysis using 3D models. Figure taken from [76]. . . . . . . . . . . . . 69

3.15 A Schematic representation of the Far Detector DAQ system. Figure taken from [77]. . . . . . . . . . . . . . 70

3.16 Feynman diagram for a $v_{\mu}$ CC interaction (left). The corresponding reconstructed event in the MINOS detectors, a long muon track is clearly visible. The dots represent the reconstructed position of the scintillator strip with different energy deposition (right). . . . . . . . . . . . . . . 72 
3.17 Feynman diagram for a NC interaction (left). The corresponding reconstructed event in the MINOS detectors, the hadronic shower is clearly visible. The dots represent the reconstructed position of the scintillator strip with different energy deposition (right). . . . . . . . . . . . . . 72

3.18 Feynman diagram for a $v_{\mathrm{e}}$ interaction (left). The corresponding reconstructed event in the MINOS detectors, the signature is a compact electromagnetic shower. The dots represent the reconstructed position of the scintillator strip with different energy deposition (right). . . . . . . . . . . . 73

4.1 One beam spill observed in the Near Detector. Neutrinos are incident from the left and only the upstream section of the detector is shown. Grey vertical bars indicate the scintillator coverage. The timing and topological pattern of hits in the detector has been used to reconstruct and select an event containing a $5.6 \mathrm{GeV}$ muon and a $2.6 \mathrm{GeV}$ hadronic shower. Beam's eye view of the detector is shown in (b), along with the reconstructed horizontal and vertical positions of track hits in each detector plane. Figure (c) shows the detector signal as a function of time, with signals from the selected event shaded [81]. . . . . . . . . . . . . 77

4.2 Cartoon of the kNN algorithm. Training points neighbouring the input point (the star) and their selected energies (represented by the diameters) are averaged. . . . . . . . 80

4.3 The response in MEU for the a) Near Detector and b) Far Detector as a function of time, as measured using cosmic data, before and after applying calibration corrections. Each point corresponds to one month of data and is normalized to the mean MEU value for all run periods. The calibrated MEU value over time is stable to within $0.5 \%$ for Near Detector and within 1.5\% for Far Detector. . . . . . 85

4.4 MINOS calorimetric response to pions and electrons at three momenta. The calorimeter-signal scale is in arbitrary units. The data (open symbols), obtained from the calibration detector exposure to CERN test beams are compared to distributions from Monte Carlo simulations [76]. . . . . . . . 86 
4.5 Each step corresponds to a correction factor in equation 4.3 . The correction from raw pulse height (ADC) to SigLin applies the linearity calibration, $L$ and drift correction $D$, from SigLin to SigCor the strip-to-strip calibration $S$, from SigCor to SigMap the attenuation correction $A$ and finally the absolute energy scale calibration $M$ to convert the raw signal into the standardised energy unit MEU. Figure is

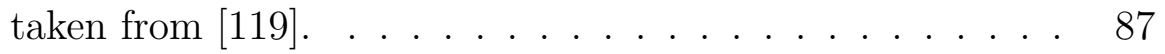

5.1 The track window used for the MEU calibration . . . . . . 92

5.2 The detector response over the time, the plots show the stability of the Far Detector in the MINOS+ era and it is with in $1 \%$. Each data point corresponds to 1 week period of data. . . . . . . . . . . . . . . . . 93

5.3 Far Detector MEU as a function of plane number, as measured using cosmic data. Individual runs outside of the run period boundaries were removed. . . . . . . . . . . 94

5.4 The detector response over the time, the plots show the stability of the Near Detector in the MINOS+ era, and it is stable with in $1.5 \%$. Each data point corresponds to 1 week period of data. . . . . . . . . . . . 95

5.5 Near Detector MEU as a function of plane number, as measured using cosmic data. Individual runs outside of the run period boundaries were removed. . . . . . . . . . 96

5.6 Detector response per plane to muon hits in the track window as a function of track zenith angle. Angular correction has been applied to remove the $\cos \theta_{z}$ dependence on detector response, and the distribution gets flattened after applying the angular correction. . . . . . . . . . . . . . . 97

5.7 MEU as a function of number of events in a snarl, in the MINOS Near Detector. It is clear that as there are more events in the snarl, MEU drops. This is studied by splitting the data set into different month. . . . . . . . . . . . . . 99

5.8 MEU as a function of number of events in a snarl, in the MINOS + era. No clear evidence that MEU is decreasing. This is studied by splitting the data set into different month.100 
5.9 The black dotted line shows the unoscillated energy spectra, and the green and red are oscillated spectra with oscillation parameter $\Delta m_{23}^{2}=0.0025 \mathrm{eV}^{2}$ and $\sin ^{2} \theta_{23}=0.95$, with $10 \%$ shift and with out shift respectively. . . . . . . . . . 102

5.10 Fractional bias for $\Delta m^{2}$ for the spectrum which oscillated with values of $\Delta m^{2}$ and fixed $\theta . \ldots \ldots \ldots 3$

5.11 Fractional bias for $\Delta m^{2}$ for the spectrum which oscillated with a fixed value of $\Delta m^{2}$ and different values of $\theta . . \ldots 103$

5.12 The ratio of MEU for different configuration as a function of X,Y and Z hit. Near Detector distribution (left) and Far Detector distribution (right). $\ldots \ldots \ldots \ldots$. . . . . 106

6.1 The probability as a function of $L / E$ for charged current (left) and neutral current events (right) for different values of $\Delta m_{41}^{2}$, the position of detector is also shown. $\ldots$. . . . 110

6.2 Shows the probability as a function of L/E. The effect of CP violating phase for $v_{\mu}$ disappearance and $v_{\mu} \rightarrow v_{s}$. For $v_{\mu}$ disappearance the effect is negligible and there is a small effect in the $v_{\mu} \rightarrow v_{s}$. We ignore these small effects. . . . . 111

6.3 Number of charged current anti-neutrino events per $10^{16}$ protons-on-target(POT) as a function of reconstructed neutrino energy in $\mathrm{GeV}$. The data are subdivided into calendar

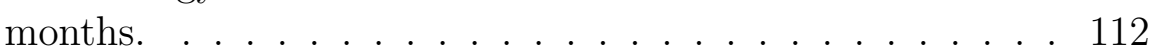

6.4 Number of charged current muon antineutrino events per $10^{16}$ protons-on-target as a function of total integrated exposure. The data are shown for two energy ranges, $<6$ $\mathrm{GeV}$ and $>6 \mathrm{GeV}$ and are subdivided into calendar months. 113

6.5 Performance of the current RHC selection (the CC and NC separation variable $(\mathrm{roID})>0.3$ and product of momentum and charge $(q p)>0)$ in the Near and Far Detector, which is essentially the CC selection. The dashed lines shows the contamination before the selection and the solid shows efficiency and contamination after the selection. The $v_{\mu}$ contamination rises at higher energies as it is difficult to assign charge for these tracks. $\ldots \ldots \ldots \ldots$. . . . . . 115 
6.6 This plot shows the percent difference between Poisson and Gaussian distribution as a function of the number of expected events in some arbitrary bin. . . . . . . . . . . . 117

6.7 Shows the re-binned spectrum for the Far Detector expected number of events (left) and Far-over-Near ratio (right). 118

6.8 Statistical Covariance matrix (left) and corresponding Error band on $F / N$ ratio (right), generated from the covariance matrix. . . . . . . . . . . . . . . . . . . . 119

6.9 The error on hadron production on $F / N$ ratio (left). And the hadron production covariance matrix (right). $\quad \ldots . .120$

6.10 The error on CC normalisation on $F / N$ ratio (left). And the covariance matrix (right). $\ldots \ldots \ldots \ldots$

6.11 The error band made for fiducial volume tightening, here the shifted distribution is constructed by tightening the fiducial radius from $80 \mathrm{~cm}$ to $60 \mathrm{~cm}$, and the ratio with the nominal is taken (left). The corresponding covariance matrix (right). . . . . . . . . . . . . . . . . . 121

6.12 Shows the mis-modeling of back exiting tracks. The shifted distribution is constructed by removing all events with a track ending with in 10 planes of the end of the Near Detector (left). And the corresponding covariance matrix (right). 122

6.13 This shows mis-modeling of the spectrometer join region. The shifted distribution is constructed by removing all events with a track ending within 10 planes of the start of the spectrometer (left). And the corresponding covariance matrix

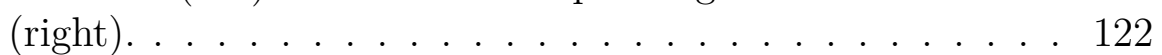

6.14 The mis-modeling of exiting tracks. The shifted distribution is constructed by turning off the containment cut (left).

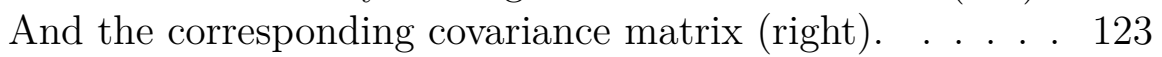

6.15 The beam optics systematics due to the horn current distribution on the $F / N$ ratio. $\ldots \ldots \ldots \ldots$. . . . . . . . 124

6.16 Cross section error band for the value of the axial vector mass $\left(M_{A}\right)$ for quasi-elastics events obtained by varying it by 1.15 and 0.85 (left). And the corresponding covariance matrix (right). . . . . . . . . . . . . . 125 
6.17 Statistical and systematics error band and for the $F / N$ ratio. The statistical error dominates due to low statistics in FD for the antineutrino CC samples. . . . . . . . . . . 125

6.18 The sensitivity for sterile neutrino at $3.36 \times 10^{20}$ POT antineutrino optimized mode running. Only CC events with positive charged track $\left(\mu^{+}\right)$are used in the analysis. $\quad$. . . 126

6.19 The sensitivity for sterile neutrino at $3.36 \times 10^{20}$ POT antineutrino optimised mode running. Only CC events with positive charged track $\left(\mu^{+}\right)$are used in the analysis. Figure shows the contribution of systematics effect on the sensitiv-

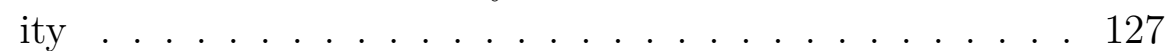

6.20 The sensitivity for sterile neutrino after including all systematics in the antineutrino optimised mode running, compared with the limit set by the other experiments. . . . . . 128

6.21 The left figure shows surface in terms of $\Delta m_{43}^{2}$ and right figure shows the surface in terms of $\Delta m_{41}^{2}$.

6.22 The surface is made in $\log z$ scale to see the the surface features at lower $\Delta m_{41}^{2}$ region. . . . . . . . . . . . . . . . 129

6.23 The $\Delta \chi_{\text {profile }}^{2}$ and $\Delta \chi_{\text {best }}^{2}$ distribution for 2500 fake experiment at $\Delta m_{41}^{2}=0.5 \mathrm{eV}^{2}$ and $\theta_{24}=0.2$, the $\Delta \chi_{\text {best }}^{2}$ is obtained by doing a 5 parameter fit where we allow both sterile and atmospheric parameters are allowed to be free. .131

6.24 The $\Delta \chi^{2}$ distribution for 2500 fake experiment, here we are getting the up value for $90 \%$ C.L for 5.22, ideally it should be 4.61 for $90 \%$ C.L for 2 degrees of freedom. . . . . . . . 131

6.25 Distribution of track vertex for the $\bar{v}_{\mu}$ events in the MINOS Far Detector. A total of 226 events were observed after opening the data. . . . . . . . . . . . . . . . . . . 132

6.26 The far detector energy spectrum with three flavour prediction and with null oscillation are compared. . . . . . . 133

6.27 The $F / N$ ratio using data (left) and the double ratio(right) plots are shown, where the best fit values obtained from the fit are compared with data and three flavour prediction. . . 133

6.28 The $90 \%$ data limit obtained from antineutrino mode running. Everything right of the curve is excluded. . . . . . . 134

6.29 The $\Delta \chi^{2}$ surface generated for RHC data surface. The zaxis shows the value of $\Delta \chi^{2}$ at corresponding grid point. $\quad 135$ 
6.30 The raw limit and Feldman-Cousins limit. The surface is converted into $\sin ^{2} 2 \theta_{24} \ldots \ldots \ldots \ldots \ldots$. . . . . . . 135

6.31 The Combined Bugey and MINOS limit shown in terms of appearance angle, which is same as that of probed by LSND and MiniBooNE. . . . . . . . . . . . . . . . . . 137

6.32 The combined MINOS and MINOS+ projected sensitivity for the antineutrino in MINOS+ era for a PoT of $4.5 \times 10^{20}$ (basically estimated for a year of running). The MINOS sensitivity is also shown. . . . . . . . . . . 138

6.33 The combined MINOS and MINOS+ projected sensitivity for the antineutrino in MINOS+ era for a PoT of $9.0 \times 10^{20}$ (basically estimated for two year of running). The MINOS sensitivity is also shown. . . . . . . . . . . . 138

6.34 The combined Bugey and MINOS+ projected sensitivity for the antineutrino in MINOS+ era for a PoT of $4.5 \times 10^{20} .139$

A.1 Schematic representation of neutrino parents in the NuMI decay pipe, illustrating the different solid angles subtended by the Near and Far Detectors at the parent decay point.

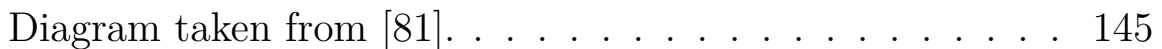

A.2 The connection between the energy of neutrinos observed in ND and neutrinos observed in FD. Decays producing neutrinos with a given energy in the Near Detector would produce a range of energies in the Far Detector, yielding the energy smearing seen here. Figure taken from [81]. . . 145

A.3 The joint distribution of neutrino energies observed in the Near and Far Detector. The contents of each cell represent the mean number of $v_{\mu}$ events expected in the Far Detector for one event in the Near Detector. This distribution may be treated as a matrix which relate the energy spectra measured in the Near Detector to those in the Far Detector [81.146

A.4 The procedure for the beam matrix extrapolation to get the FD energy spectrum from the ND reconstructed energy spectrum. Figure taken from [69]. . . . . . . . . . . . . . 147 
B.1 The Near Detector pre-selection variables a) shows the DataMC comparison of the maximum consecutive plane variable, the contribution from the poorly reconstructed events are also show b) shows the Data-MC comparison of the slice pulse height fraction, the poorly reconstructed component of the events are also shown. . . . . . . . . . . . . . 151

B.2 The error band for the ND preselection cleaning systematic is shown. The values of the systematic errors assign on the first $2 \mathrm{GeV}$ bins are: $12.1 \%, 9.7 \%, 6.9 \%$ and $4.7 \%$. The red line corresponds to the $+1 \sigma$, the blue line to the $-1 \sigma$. The error band is symmetric by constructions. . . . . . . . . . 152

B.3 Data-MC Comparisons of the neutral current classification variable a) Event length and b) Track Extension. Data and MC shows good agreement, the CC background events are also shown. . . . . . . . . . . . . . . . . 153

B.4 Data and MC distributions and ratios for the vertex positions of NC selected events. Fiducial and cleaning cuts are applied along with NC selection cuts. a) Vertex X b) Vertex Y and c) Vertex Z. . . . . . . . . . . . . . . 154

B.5 a) The efficiency (red) and purity (blue) for the neutral current selection as a function of energy. b) Data-MC comparison of the selected ND neutral current energy spectrum. The bad data-MC agreement in the first two energy bin are due to the presence of the poorly reconstructed events. . . 155

B.6 The total neutral current energy spectra are split up into different component. The CC background is also shown. Because of the low background below $5 \mathrm{GeV}$, it gives better sensitivity for the sterile neutrino search. . . . . . . . . . 156 


\section{1 \\ Introduction to Neutrinos}

Neutrinos are the second most abundant particle in the universe after photons. The $\beta$-decay study in early 1900's provided the first evidence for the neutrinos. In 1911, Lise Meitner and Otto Hahn showed that the energy of electrons coming out from the $\beta$-decay is continuous rather than discrete energy spectrum. This was a contradiction to the laws of conservation of energy. In 1930 December, Wolfgang Pauli proposed a new particle as an immediate remedy for the observed continuous $e^{-}$energy spectrum in the $\beta$-decay [1]. In a letter addressing his colleagues, in a conference at Tübingen, he proposed a "desperate remedy to save the exchange theorem of statistics and the law of conservation of energy" [2]. Pauli described this new particle as a charge less $1 / 2$ - spin particle, he called it as "neutron" as it is chargeless. He proposed that a "neutron" is also emitted along with the electron such that the the sum of the "neutron" and $e^{-}$energy is constant, which explained the continuous nature of the electron energy spectra observed in the $\beta$-decay. In 1931, Fermi renamed Pauli's "neutron" and he gave the name neutrino which means small and neutral in Italian. This elusive particle has continued to be a fascinating object to physicists since then.

\subsubsection{The Weak Interaction}

Neutrino interacts via weak nuclear force. Fermi introduced the theory of weak interaction in analogy with electromagnetic interaction [3]. This interaction is also know as four fermion contact interaction which involves electron, proton, neutron and neutrino coupling, the matrix element for the $\beta$-decay can be expressed as,

$$
M=G\left(\bar{u}_{n} \gamma^{\mu} u_{p}\right)\left(\bar{u}_{\gamma} \gamma_{\mu} u_{e}\right) .
$$


where, $G$ is the coupling constant, $u_{i}$ is the Dirac spinor of the particle $i$, and $\gamma_{\mu}$ 's are the Dirac gamma matrices. This interaction contains no propagator and happens at a point. Fermi assumed that the parity is conserved in this process and the form of reaction is vector-vector coupling. Over the time it was understood that it requires some modification to the Fermi's theory, especially after the discovery of parity violation. Many papers questioned the vector-vector nature of the interaction and led to the inclusion of the axial component into the theory ( $V-A$ theory) and the matrix elements get modified accordingly [4, 5]. The resulting $\beta$-decay matrix element is

$$
M=\frac{G}{\sqrt{2}}\left[\bar{u}_{n} \gamma^{\mu}\left(1-\gamma^{5}\right) u_{p}\right]\left[\bar{u}_{\nu} \gamma_{\mu}\left(1-\gamma^{5}\right) u_{e}\right] .
$$

This theory explained most of the weak interaction phenomena at low energy, but the theory has difficulties as a relativistic field theory. Theory predicts the cross section for a neutrino neutron interaction, $\sigma(v n) \propto E_{v}^{2}$ ( $E_{v}$ is the neutrino energy) at low energy, but violates unitarity around 300 $\mathrm{GeV}$ [6] (first noticed by Heisenberg in 1936 [7]). The problem is that the theory is not-renormalisable. The mathematical problems of introducing a massive boson and making the theory renormalisable was established by Sheldon Glashow, Abdus Salam, and Steven Weinberg [8 10]. The consequences of the theory were the presence of neutral current component to the weak interaction and masses for exchange particles $W^{ \pm}$and $Z^{0}$.

The neutral current interaction was discovered in the Gargamelle bubble chamber in 1973 [11, 12]. The masses of the exchange bosons $W^{ \pm}$and $Z^{0}$ were first measured at the UA1 and UA2 experiments at the $p \bar{p}$ collider at CERN [13-16]. The Feynman diagram for neutral current (mediated via $Z^{0}$ ) and charged current (mediated via $W^{ \pm}$) interaction is displayed in Fig. 1.1. 

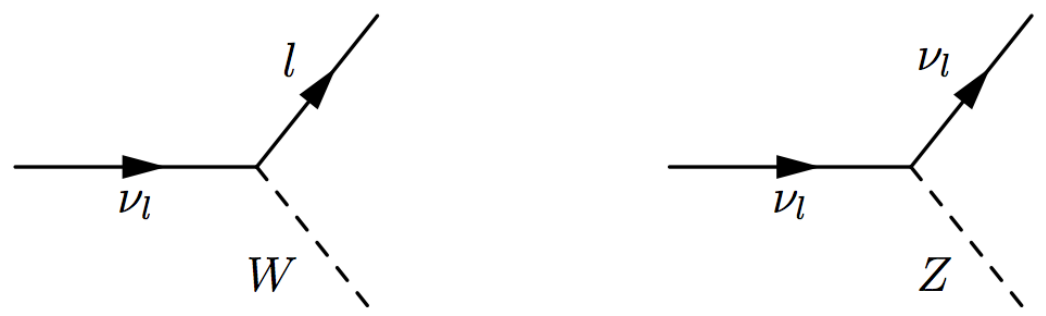

Figure 1.1: Two possible types of weak interaction. The charged current interaction (left) via $W$ boson exchange and neutral current interaction (right) via exchange of $Z^{0}$. This is applicable to both neutrinos and antineutrinos.

\subsubsection{Three types of Neutrinos}

In 1956, Reines and Cowan experimentally discovered first neutrino through the inverse beta decay [17], they used the $\bar{v}_{e}$ from the reactor and collided it with protons to produce neutrons and positrons, $\overline{\mathrm{v}}_{e}+\mathrm{p} \rightarrow \mathrm{n}+\mathrm{e}^{+}$ (Nobel prize for the year 1995 was awarded to Reines and Cowan for this discovery).

In 1962, an experiment at Brookhaven discovered neutrino which is produced by the pion decay, which when interact with the detector produced muons, not electrons. This experiment was an indication that there exists a second generation of neutrino. Leon Lederman, Schwartz and Steinberger [18] confirmed the existence of this second generation neutrinos. In 1988 Nobel prize was awarded for this discovery of muon neutrino. The third generation of neutrinos, called tau neutrino $\left(v_{\tau}\right)$, was discovered in 2000 at Fermilab's DONUT (Direct Observation of NU Tau) experiment [19].

The number of neutrino generations is same as the number of lepton generations and this has been widely accepted and has been included in the Standard Neutrino Model (SvM) [20]. The LEP (Large Electron-Positron) collider experiment at CERN confirmed that there are only three active neutrinos by measuring the width of $Z^{0}$ boson [21]. Cosmological data also suggests that there are three active neutrinos [22]. 


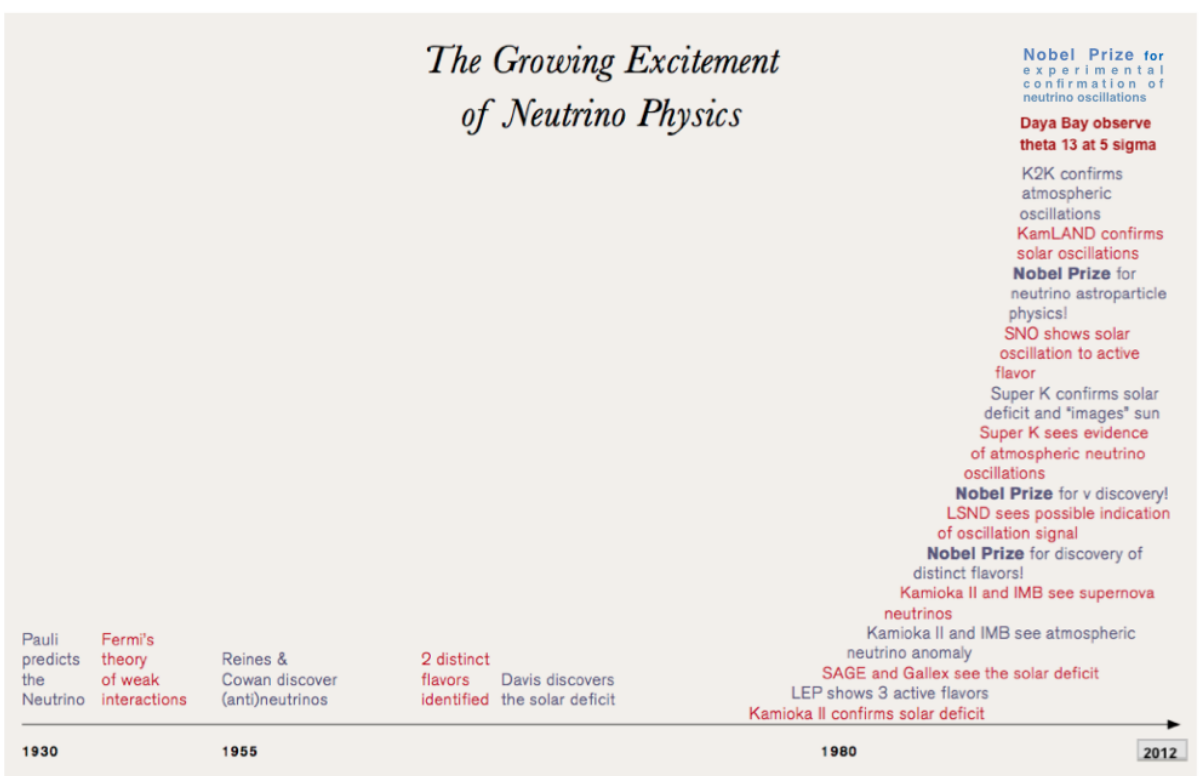

Figure 1.2: A history of neutrino physics in brief. Figure is taken from [23].

\subsubsection{Neutrino Flavour Change and Mass}

The mass of neutrinos had not been measured and there were many thoughts that it may be identically zero in late 1950's. Burno Pontecorvo came up with the idea of neutrino flavour change, which is allowed by nonzero neutrino masses [24]. At that time only one neutrino was known, $v_{e}$, so the oscillations considered were $v \rightleftharpoons \bar{v}$ oscillations by analogy with the kaon system, assuming there is no lepton number violation in the neutrino sector. After the discovery of $v_{\mu}$ in 1967, he considered the possibility of neutrino flavour change including $v_{\mu} \rightleftharpoons v_{e}$ oscillations. In 1964, Ziro Maki, Masami Nakagawa and Shoichi Sakata discussed the two-flavour oscillation model in the form that we know of today [25].

\subsection{Neutrino Oscillations}

Neutrino oscillation is a quantum mechanical phenomena, which manifests the flavour changes in neutrinos. The mathematical formalism for 
the neutrino oscillation was put forward by Pontecarvo, Maki, Nakagawa, and Sakata. Neutrinos are generated in a weak interaction, as the flavour eigenstate, $\left|v_{e}\right\rangle,\left|v_{\mu}\right\rangle$, and $\left|v_{\tau}\right\rangle$ and it propagate as the mass eigenstate $\left|v_{1}\right\rangle,\left|v_{2}\right\rangle$ and $\left|v_{3}\right\rangle$. These two sets of states are connected by a unitary matrix called PMNS matrix, $U$ such that

$$
\left(\begin{array}{l}
v_{e} \\
v_{\mu} \\
v_{\tau}
\end{array}\right)=U^{*}\left(\begin{array}{l}
v_{1} \\
v_{2} \\
v_{3}
\end{array}\right)
$$

and $U^{\dagger} U=\mathbb{I}$. In the standard parametrization, the mixing matrix is described by three mixing angles $\theta_{12}, \theta_{23}, \theta_{13}$ and a $C P$-violating phase, $\delta$ (Dirac phase) and the matrix can be written as,

$$
U=\left(\begin{array}{ccc}
U_{e 1} & U_{e 2} & U_{e 3} \\
U_{\mu 1} & U_{\mu 2} & U_{\mu 3} \\
U_{\tau 1} & U_{\tau 2} & U_{\tau 3}
\end{array}\right)
$$

This matrix can be written as in terms of mixing angle and the CPviolating phase as,

$$
\begin{aligned}
U & =\left(\begin{array}{ccc}
1 & 0 & 0 \\
0 & c_{23} & s_{23} \\
0 & -s_{23} & c_{23}
\end{array}\right)\left(\begin{array}{ccc}
c_{13} & 0 & s_{13} e^{-i \delta} \\
0 & 1 & 0 \\
-s_{13} e^{-i \delta} & 0 & c_{13}
\end{array}\right)\left(\begin{array}{ccc}
c_{12} & s_{12} & 0 \\
-s_{12} & c_{12} & 0 \\
0 & 0 & 1
\end{array}\right) \\
U & =\left(\begin{array}{ccc}
c_{12} c_{13} & s_{12} c_{13} & s_{13} e^{-i \delta} \\
-s_{12} c_{23}-c_{12} s_{23} s_{13} e^{i \delta} & c_{12} c_{23}-s_{12} s_{23} s_{13} e^{i \delta} & s_{23} c_{13} \\
s_{12} s_{23}-c_{12} c_{23} s_{13} e^{i \delta} & -c_{12} s_{23}-s_{12} c_{23} s_{13} e^{i \delta} & c_{23} c_{13}
\end{array}\right)
\end{aligned}
$$

where, $s_{i j}=\sin \theta_{i j}$ and $c_{i j}=\cos \theta_{i j}$. Now a neutrino of definite flavour $v_{\alpha}$ can be written as a coherent superposition of mass eigenstates $v_{i}$,

$$
\left|v_{\alpha}(0)\right\rangle=\sum_{i} \mathrm{U}_{\alpha i}^{*}\left|v_{i}\right\rangle .
$$

In course of time, this neutrino state evolves into a state associated with a phase and the neutrino state at a later time $t$ is given by,

$$
\left|\boldsymbol{v}_{\alpha}(t)\right\rangle=\sum_{i} \mathrm{U}_{\alpha i}^{*} e^{i p_{i} \cdot x}\left|\boldsymbol{v}_{i}\right\rangle
$$


with $p_{i} \cdot x=p_{i}^{\mu} \cdot x_{\mu}$, where $x_{\mu}$ is the space time four vector and $p_{i}^{\mu}$ is the four momentum of the mass eigenstates $i$. When neutrino interact with the detector the wave function collapses and the transition amplitude of a neutrino of flavour $\alpha$ at $(0,0)$ to be detected as a neutrino of flavour $\beta$ at later space time point $(\vec{x}, t)$ can be written as,

$$
\left\langle v_{\beta} \mid v_{\alpha(t)}\right\rangle=\sum_{j} \sum_{i} \mathrm{U}_{\beta j} \mathrm{U}_{\alpha i}^{*} e^{i p_{i} \cdot x}\left\langle v_{j} \mid v_{i}\right\rangle
$$

by performing sum over all $\mathrm{j}$ we get

$$
\left\langle v_{\beta} \mid v_{\alpha(t)}\right\rangle=\sum_{i} \mathrm{U}_{\beta i} \mathrm{U}_{\alpha i}^{*} e^{i p_{i} \cdot x}
$$

The energy and mass of the $i^{t h}$ neutrino mass eigenstate $E_{i}$ and $m_{i}$ respectively. By assuming all the mass eigenstates have same three-momentum $\vec{p}$, we have

$$
\begin{aligned}
& p_{i} \cdot x=E_{i} t-\vec{p} \cdot \vec{x}, \\
= & t \sqrt{|p|^{2}+m_{i}^{2}}-\vec{p} \cdot \vec{x} .
\end{aligned}
$$

After performing the expansion of the above term and assuming ( $m_{i} \ll$ $E_{i}$ ), for relativistic neutrino we can take, $\mathrm{t}=\mathrm{L}$ and $\vec{p} \cdot \vec{x}=|\vec{p}| \mathrm{L}$ (where, $\mathrm{L}$ is the distance travelled), we obtain

$$
p_{i} \cdot x=|p| L\left(1+\frac{m_{i}^{2}}{2|p|^{2}}\right)-|p| L
$$

Taking $E$ as the average energy, $m_{i} \ll E_{i}$ and $|\vec{p}| \approx E$, we can write

$$
p_{i} \cdot x=\frac{m_{i} L}{2 E},
$$

thus

$$
\left\langle v_{\beta} \mid v_{\alpha(L)}\right\rangle=\sum_{i} \mathrm{U}_{\beta j} \mathrm{U}_{\alpha i}^{*} e^{i \frac{m_{i}^{2}}{2 E}}
$$

The probability of observing a neutrino flavour of $v_{\beta}$ a distance $L$ from the source, from a pure $v_{\alpha}$ at the source is given by,

$$
P\left(v_{\alpha} \rightarrow v_{\beta}\right)=\left|\left\langle v_{\beta} \mid v_{\alpha(L)}\right\rangle\right|^{2},
$$




$$
\begin{aligned}
P\left(v_{\alpha} \rightarrow v_{\beta}\right)= & \left(\sum_{j} \mathrm{U}_{\beta j}^{*} \mathrm{U}_{\alpha j} e^{-i \frac{m_{j}^{2} L}{2 E}}\right)\left(\sum_{j} \mathrm{U}_{\beta i} \mathrm{U}_{\alpha i}^{*} e^{-i \frac{m_{i}^{2} L}{2 E}}\right) \\
P\left(v_{\alpha} \rightarrow v_{\beta}\right)= & \sum_{i} \sum_{j} \mathrm{U}_{\beta j}^{*} \mathrm{U}_{\beta i} \mathrm{U}_{\alpha i}^{*} \mathrm{U}_{\alpha j} e^{-i \frac{\Delta m_{i j}^{2} L}{2 E}}+ \\
& {\left[\sum_{i} \sum_{j} \mathrm{U}_{\beta j}^{*} \mathrm{U}_{\beta i} \mathrm{U}_{\alpha i}^{*} \mathrm{U}_{\alpha j}-\sum_{i} \sum_{j} \mathrm{U}_{\beta j}^{*} \mathrm{U}_{\beta i} \mathrm{U}_{\alpha i}^{*} \mathrm{U}_{\alpha j}\right], }
\end{aligned}
$$

where, $\Delta m_{i j}^{2}=m_{i}^{2}-m_{j}^{2}$. The last terms are identical and the sum becomes zero. For the easy interpretation $\Delta m_{i j}^{2}=m_{i}^{2}-m_{j}^{2}$ is known as the neutrino mass splitting and the above equation becomes,

$$
\begin{aligned}
P\left(v_{\alpha} \rightarrow v_{\beta}\right)= & \sum_{i} \sum_{j} \mathrm{U}_{\beta j}^{*} \mathrm{U}_{\beta i} \mathrm{U}_{\alpha i}^{*} \mathrm{U}_{\alpha j}\left(e^{-i \frac{\Delta m_{i j}^{2} L}{2 E}}-1\right) \\
& +\sum_{i} \sum_{j} \mathrm{U}_{\beta j}^{*} \mathrm{U}_{\beta i} \mathrm{U}_{\alpha i}^{*} \mathrm{U}_{\alpha j} .
\end{aligned}
$$

The second term in the above equation can be simplify as,

$$
\begin{aligned}
\sum_{i} \sum_{j} \mathrm{U}_{\beta j}^{*} \mathrm{U}_{\beta i} \mathrm{U}_{\alpha i}^{*} \mathrm{U}_{\alpha j} & =\sum_{i} \mathrm{U}_{\beta i} \mathrm{U}_{\alpha i}^{*} \sum_{j} \mathrm{U}_{\beta j}^{*} \mathrm{U}_{\alpha j} \\
& =\sum_{i} \mathrm{U}_{\beta i} U_{i \alpha}^{\dagger} \sum_{j} U_{j \beta}^{\dagger} \mathrm{U}_{\alpha j} \\
& =\delta_{\alpha \beta} .
\end{aligned}
$$

because of the unitarity of $U$, if we take the first term as $B_{i j}$, then $B_{i j}=B_{i j}^{*}$ thus $B_{i j}+B_{j i}=2 \Re\left[B_{i j}\right]$ and equation 1.15 leads to

$$
P\left(v_{\alpha} \rightarrow v_{\beta}\right)=\delta_{\alpha \beta}+2 \sum_{i(>j)} \sum_{j} \Re\left[\mathrm{U}_{\beta j}^{*} \mathrm{U}_{\beta i} \mathrm{U}_{\alpha i}^{*} \mathrm{U}_{\alpha j}\left(e^{-i \frac{\Delta m_{i j}^{2} L}{2 E}}-1\right)\right]
$$

Using the identity

$$
e^{-i \frac{\Delta m_{i j}^{2} L}{2 E}}=\cos \left(\frac{\Delta m_{i j}^{2} L}{2 E}\right)+i \sin \left(\frac{\Delta m_{i j}^{2} L}{2 E}\right)
$$


and

$$
\cos \left(\frac{\Delta m_{i j}^{2} L}{2 E}\right)-1=2 \sin ^{2}\left(\frac{\Delta m_{i j}^{2} L}{4 E}\right)
$$

the probability can be written as,

$$
\begin{aligned}
P\left(v_{\alpha} \rightarrow v_{\beta}\right)=\delta_{\alpha \beta} & \\
& +2 \sum_{i(>j)} \sum_{j} \operatorname{Im}\left[\mathrm{U}_{\beta j}^{*} \mathrm{U}_{\beta i} \mathrm{U}_{\alpha i}^{*} \mathrm{U}_{\alpha j}\right] \sin \left(\frac{\Delta m_{i j}^{2} L}{2 E}\right) \\
& -4 \sum_{i(>j)} \sum_{j} \Re\left[\mathrm{U}_{\beta j}^{*} \mathrm{U}_{\beta i} \mathrm{U}_{\alpha i}^{*} \mathrm{U}_{\alpha j}\right] \sin \left(\frac{\Delta m_{i j}^{2} L}{4 E}\right)
\end{aligned}
$$

\subsection{The Two Flavour Approximation}

The two-neutrino flavour approximation is a fairly accurate description for a number of neutrino oscillation experiments. For simplicity, we consider that neutrinos exist only in two flavours. Now in equation 1.21, the argument of the last sinusoidal term with the imaginary part can be written in S.I unit as,

$$
\frac{\Delta m_{i j}^{2} c^{4} L}{4 E \hbar c}
$$

Using energy in the units of $\mathrm{GeV}, L$ in $\mathrm{km}$ and $\Delta m_{i j}^{2}$ in $\mathrm{eV}^{2}$, it becomes

$$
\frac{1.27 \Delta m_{i j}^{2} L}{E} \text {. }
$$

The argument of the sinusoidal term with the real part is a factor of 2 larger. The survival probability for a muon neutrino of energy $E$ after traveling a distance $L$ is given by,

$$
P\left(v_{\mu} \rightarrow v_{\mu}\right)=1-4 \sum_{i(>j)} \sum_{j}\left|U_{\mu i}\right|^{2}\left|U_{\mu j}\right|^{2} \sin ^{2}\left(\frac{1.27 \Delta m_{i j}^{2} L}{E}\right) .
$$

The imaginary term in equation 1.21 is zero when $\alpha=\beta$. For two flavour approximation we take $\sin \theta_{13}=0$ and $\cos \theta_{13}=1$, the relevant matrix elements in the equation are, 


$$
\begin{array}{ll}
U_{\mu 1}=s_{12} c_{23}-c_{12} s_{23} s_{13} e^{i \delta} & \Longrightarrow\left|U_{\mu 1}\right|^{2} \approx s_{12}^{2} c_{23}^{2}, \\
U_{\mu 2}=c_{12} c_{23}-s_{12} s_{23} s_{13} e^{i \delta} & \Longrightarrow\left|U_{\mu 2}\right|^{2} \approx c_{12}^{2} c_{23}^{2}, \\
U_{\mu 3}=s_{23} c_{13} & \Longrightarrow\left|U_{\mu 2}\right|^{2} \approx s_{23}^{2} .
\end{array}
$$

The experiments have shown that $\Delta m_{12}^{2}=\mathcal{O}\left(7 \times 10^{-5} \mathrm{eV}^{2}\right)$. For the case of MINOS (Main Injector Neutrino Oscillations Search) experiment. The details of the experiment are described in Chapter 3, Its baseline is $735 \mathrm{~km}$, and the neutrino energy is $3 \mathrm{GeV}$, so the argument of sin function becomes,

$$
\sin ^{2}\left(\frac{1.27 \Delta m_{21}^{2} L}{E}\right) \approx \sin ^{2}\left(\frac{1.27 \times 7 \times 10^{-5} \times 735}{3}\right) \approx \sin ^{2}(0.02) \approx 0 .
$$

Similarly, $\Delta m_{31}^{2}=\mathcal{O}\left(3 \times 10^{-3} \mathrm{eV}^{2}\left(\gg \Delta m_{21}^{2}\right)\right)$ and $\Delta m_{31}^{2}-\Delta m_{21}^{2}=\Delta m_{32}^{2}$, so $\Delta m_{31}^{2} \approx \Delta m_{32}^{2}=\Delta m_{\text {atm }}^{2}$, therefore,

$$
P\left(v_{\mu} \rightarrow v_{\mu}\right) \approx 1-4 s_{23}^{2} c_{23}^{2}\left(s_{12}^{2}+c_{12}^{2}\right) \sin ^{2}\left(\frac{1.27 \Delta m^{2} L}{E}\right) .
$$

Using $2 \sin \theta_{23} \cos \theta_{23}=\sin 2 \theta_{23}$ and $\sin ^{2} \theta_{12}+\cos ^{2} \theta_{12}=1$, in the two flavour approximation the survival probability becomes,

$$
P\left(v_{\mu} \rightarrow v_{\mu}\right)=1-\sin ^{2}\left(2 \theta_{23}\right) \sin ^{2}\left(\frac{1.27 \Delta m^{2} L}{E}\right) .
$$

\section{- Electron neutrino disappearance}

The reactor experiments like Daya Bay, RENO, Double Chooz experiments [26, 27, 46] are sensitive to the channel given by

$$
\begin{aligned}
. P\left(v_{e} \rightarrow v_{e}\right) & \approx 1-4\left|U_{e 3}\right|^{2}\left(1-\left|U_{e 3}\right|^{2}\right) \sin ^{2}\left(\frac{1.27 \Delta m_{32}^{2} L}{E}\right) . \\
& \approx 1-\sin ^{2} 2 \theta_{13} \sin ^{2}\left(\frac{1.27 \Delta m_{32}^{2} L}{E}\right) .
\end{aligned}
$$

\section{- Muon neutrino disappearance}


MINOS, T2K, and NO $\nu \mathrm{A}$ are sensitive to [29, 30].

$$
\begin{aligned}
P\left(v_{\mu} \rightarrow v_{\mu}\right) & \approx 1-4\left|U_{\mu 3}\right|^{2}\left(1-\left|U_{\mu 3}\right|^{2}\right) \sin ^{2}\left(\frac{1.27 \Delta m_{32}^{2} L}{E}\right) . \\
& \approx 1-\cos ^{2} \theta_{13} \sin ^{2} 2 \theta_{23} \sin ^{2}\left(\frac{1.27 \Delta m_{32}^{2} L}{E}\right) .
\end{aligned}
$$

\section{- Electron neutrino appearance}

MINOS, T2K, and $\mathrm{NO} \nu \mathrm{A}$ are sensitive to this channel also [30, 31].

$$
\begin{aligned}
P\left(v_{\mu} \rightarrow v_{e}\right) & \approx 1-4\left|U_{\mu 3}\right|^{2}\left|U_{\mu 3}\right|^{2} \sin ^{2}\left(\frac{1.27 \Delta m_{32}^{2} L}{E}\right) \\
& \approx \sin ^{2} 2 \theta_{13} \sin ^{2} \theta_{23} \sin ^{2}\left(\frac{1.27 \Delta m_{32}^{2} L}{E}\right)
\end{aligned}
$$

\subsection{Experimental evidence for Neutrino os- cillations}

\subsubsection{Solar Neutrinos}

The first evidence for the neutrinos oscillation came from the solar neutrino studies performed by Ray Davis in 1968, at the Homestake mine. The experiment measured the flux of neutrinos coming from the ${ }^{8} \mathrm{~B}$ decay,

$$
{ }^{8} \mathrm{~B} \rightarrow{ }^{8} \mathrm{~B}^{*}+e^{+}+v_{e}(\approx 10 \mathrm{MeV})
$$

The detector element, $\mathrm{C}_{2} \mathrm{Cl}_{2}$, filled in a tank and the every time when neutrino interacts inside the detector an argon atom is produced through the reaction, $v_{e}+{ }^{37} \mathrm{Cl} \rightarrow e^{-}+{ }^{37} \mathrm{Ar}$. The number of ${ }^{37} \mathrm{Ar}$ produced was half less than what was expected from the solar neutrino flux models [32]. At first it was thought that there can be problems with the standard solar model (SSM). An independent measurement was performed by Kamiokande using the Cherenkov detector and observed similar discrepancies in atmospheric neutrinos as well [33] . 


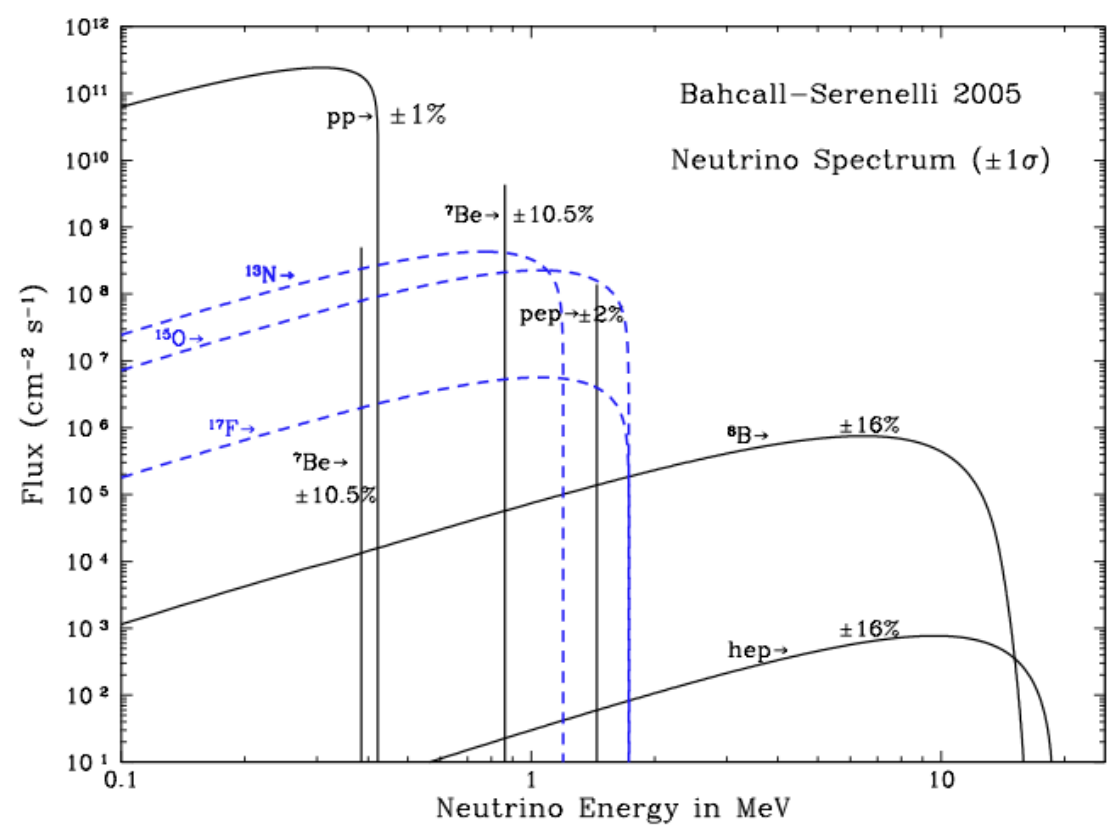

Figure 1.3: The predicted solar neutrino flux by SSM. The numbers shown are the uncertainty from the models.

Fig. 1.3 shows the neutrino flux coming from the different chain of the reaction in the sun. If we measure the neutrino flux coming out of the $p p$ chain, which is basically the fusion of hydrogen nuclei into helium,

$$
p+p \rightarrow d+e^{+}+v_{e}(\approx 0.3 \mathrm{MeV})
$$

This reaction is well studied by the radio chemical experiments like SAGE [34, GALLEX-GNO [35]. These neutrinos are studied using their interaction with Gallium,

$$
v_{e}+{ }^{57} \mathrm{Ga} \rightarrow e^{-}+{ }^{71} \mathrm{Ge}
$$

The threshold of this reaction is about $0.233 \mathrm{MeV}$. The reaction rate of this process is model independent unlike that of ${ }^{8} \mathrm{~B}$ chain. The predicted rate using the SSM is 128 SNU on Ga, but the SAGE and GALLEX observed only $70.8_{-6.1}^{+6.5}$ SNU and $77.5_{-7.8}^{+7.5}$ SNU respectively (SNU stands for Solar 
Neutrino Unit, which is $10^{36}$ events/atom/second). This is also half of what we expect from the model prediction. This problem is also known as "Solar Neutrino Problem" and later it is identified to be a consequence of neutrino oscillation.

The Sudbury Neutrino Observatory (SNO) experiment measured the solar neutrino oscillation parameter in the parameter space of $\Delta m_{21}^{2}-\theta_{12}$ [36]. The SNO is located about $2 \mathrm{~km}$ underground in a mine in Sudbury, Ontario, Canada. It is a water Cherenkov detector designed to detect the solar neutrinos with $\mathrm{D}_{2} \mathrm{O}$ as the detecting material. The experiment measured the charged and neutral current events separately. This way a model-independent test can be done to verify the neutrino oscillation hypothesis. The experiment measures the Large Mixing Angle (LMA) solution with the Mikheyev-Smirnov-Wolfenstein (MSW) matter effects [37. The combined fit to all the solar neutrino data gives the best fit oscillation parameter as,

$$
\Delta m_{21}^{2}=\left(5.6_{-1.4}^{+1.9}\right) \times 10^{-5} \mathrm{eV}^{2}, \quad \tan ^{2} \theta_{12}=0.427_{-0.029}^{+0.033} .
$$

From the the MSW effect it can be established that $m_{2}>m_{1}$ [38. There are other experiments which measure the neutrino oscillation in the "solar sector' (i.e, measure the small mass squared splitting $\Delta m_{21}^{2}$ and $\theta_{12}$ ), by studying the $v_{e}$ from other sources. Nuclear reactor is one of the source which provides a large flux of $\bar{v}_{e}$ and KamLAND is the first experiment which studied the reactor antineutrinos [39]. KamLAND detector is in the Kamioka mine in Japan, detecting element consists of 1 kTon of liquid scintillator surrounded by non-scintillating buffer oil. The scintillator light is collected by 1,900 phototubes, the neutrinos are detected via inverse beta decay and prompt delayed double coincidence of the signal. The neutrino flux for the experiment was provided by the 55 commercial nuclear reactors at a distance varying from 140 to $210 \mathrm{~km}$ from the detector. A clear signal for $\bar{v}_{e}$ disappearance is observed which was then fitted to extract the neutrino oscillation parameter, the fit gives [39],

$$
\Delta m_{21}^{2}=\left(7.66_{-0.20}^{+0.22}\right) \times 10^{-5} \mathrm{eV}^{2}, \quad \tan ^{2} \theta_{12}=0.52_{-0.10}^{+0.16} .
$$

Fig. 1.4 shows the ratio of the expected to the measured number of neutrino events and the parameters obtained from the fitting is consistent with oscillation hypothesis. 


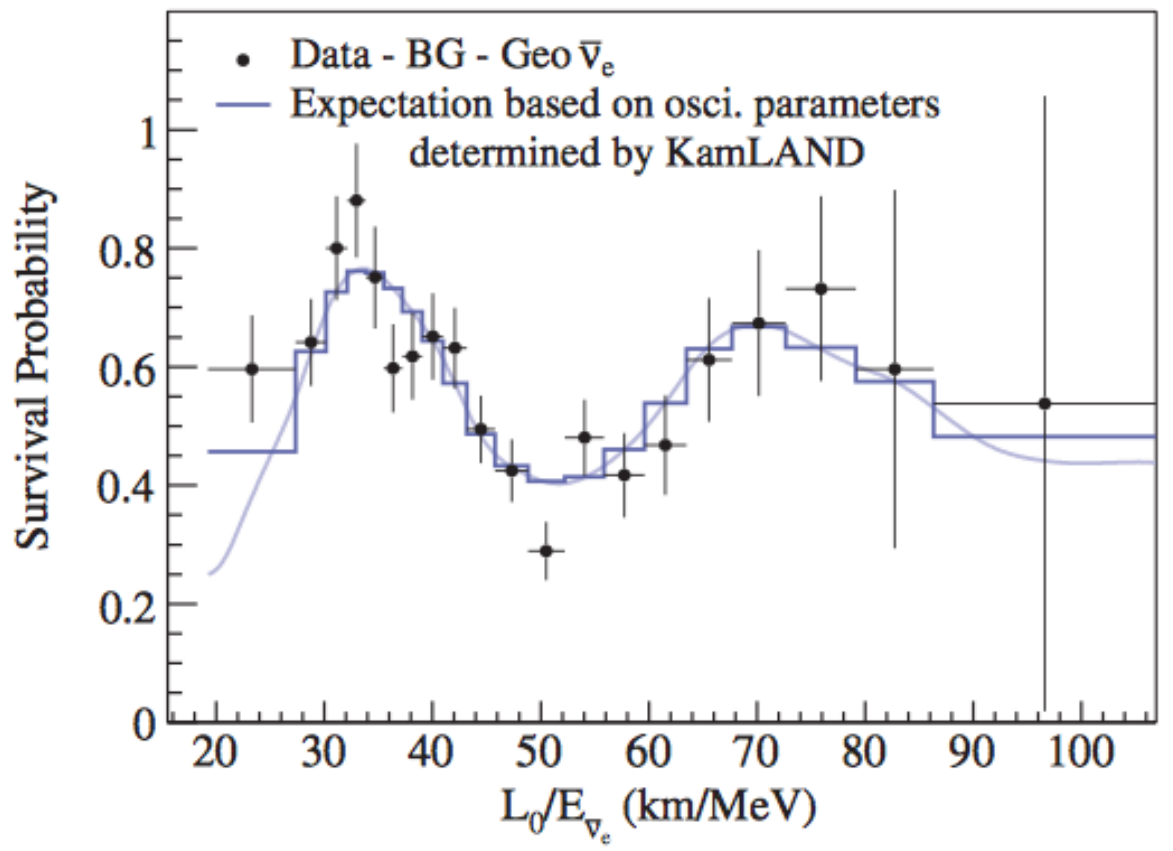

Figure 1.4: The ratio of measured rate of $\bar{v}_{e}$ to the expectation for no oscillation as function of $L / E$. Also shown the best fit for oscillation hypothesis. Figure is taken from [39].

These two independent measurements with completely different detectors brings confidence in the particle physics community that neutrinos are oscillating. The results from the solar neutrino experiment and KamLAND are combined and that yields the world's best measurement of solar neutrino parameter with a precision of $3.5 \%$ [40].

$$
\Delta m_{21}^{2}=\left(7.54_{-0.22}^{+0.26}\right) \times 10^{-5} \mathrm{eV}^{2}, \quad \tan ^{2} \theta_{12}=0.307_{-0.016}^{+0.018} .
$$




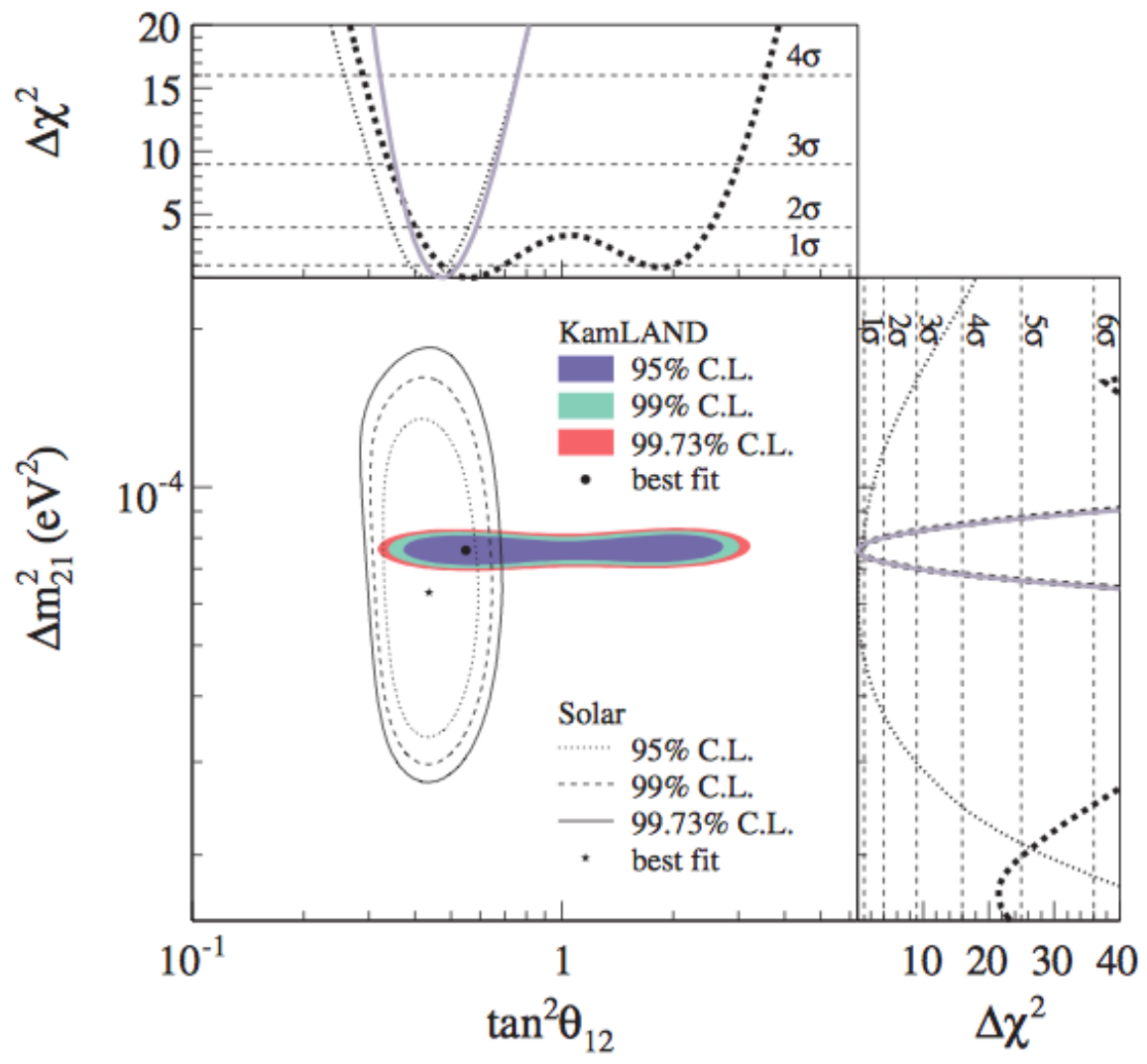

Figure 1.5: The neutrino oscillation parameters allowed by KamLAND and combined solar neutrino experiments. The side panels show the $\Delta \chi^{2}$ profiles for KamLAND (dashed line) and solar experiments (dotted line) individually, and the combination of the two (solid line). Figure is taken from [39].

\subsubsection{Atmospheric Neutrinos}

Atmospheric neutrino sector measures the mass squared splitting $\Delta m_{23}^{2}$ and mixing angle $\theta_{23}$. These parameters can be measured by studying the atmospheric neutrino oscillations and accelerator based neutrino beams $\left(v_{\mu}\right)$. Cosmic rays produce pions and kaons when they strike the earth's atmosphere, this pions and koans, which then decay and produce muon 
neutrinos.

$$
\pi^{ \pm} \rightarrow \mu^{+}+v_{\mu} \quad \mu^{+} \rightarrow e^{+}+v_{e}+\bar{v}_{\mu}
$$

If there were no neutrino oscillations, we would expect flux ratio for $v_{\mu}: v_{e}$ is $2: 1$ (given the fact that your detector cannot distinguish $\mu^{+}$and $\mu^{-}$), but the observed flux rate for $v_{\mu}$ was $2 / 3$ of the prediction [41]. The Super Kamiokande collaboration in 1998 studied the atmospheric neutrinos [42] by looking at the zenith angle distribution of charged current interaction due to the muon neutrinos and electron neutrinos.
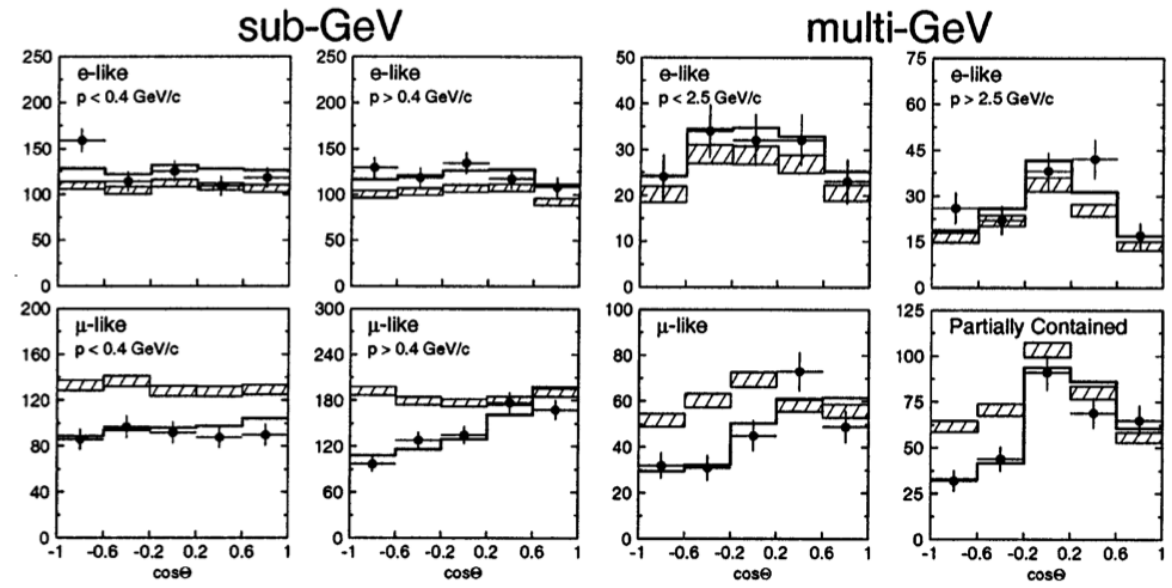

Figure 1.6: Zenith angle distributions of $\mu$-like and e-like events for sub$\mathrm{GeV}$ and multi-GeV data set. The dotted lines are the prediction from the Monte Carlo with out oscillation and the solid line is the best fit to the $v_{\mu} \rightarrow v_{\tau}$ oscillations. The sub-GeV events are with energy $<1.33 \mathrm{GeV}$, mutli-GeV events are with energy $>1.4 \mathrm{GeV}$. Plot is taken from [42]

The neutrino oscillation in atmospheric sector can also be studied using the neutrinos produced by accelerators beams. The neutrino oscillation hypothesis is independently confirmed by accelerator based muon neutrinos also. In a typical accelerator based neutrino experiment, neutrino beam energy is tuned to get the best oscillation sensitivity in the detector which is placed at a distance $L$ (one oscillation length) from the source. The first oscillation results using the accelerator based neutrino is from K2K experiment in Japan, KEK uses $1 \mathrm{GeV}$ of neutrino produced at the 
K2K accelerator and events were observed at the Super-Kamiokande detector which is $250 \mathrm{~km}$ away from the neutrino source. The most precise measurement of mass square splitting in atmospheric sector is done by MINOS and mixing angle is measured by Super-Kamiokande. The most precise measurement for $\Delta m_{23}^{2}$ [43] and $\sin ^{2} \theta_{23}$ [30] is done by these experiments and the values are,

$$
\Delta m_{32}^{2}=2.41_{-0.10}^{+0.09} \times 10^{-3} \mathrm{eV}^{2}, \quad \sin ^{2} 2 \theta_{12}=0.95_{-0.036}^{+0.035}(90 \% \text { C.L }) .
$$

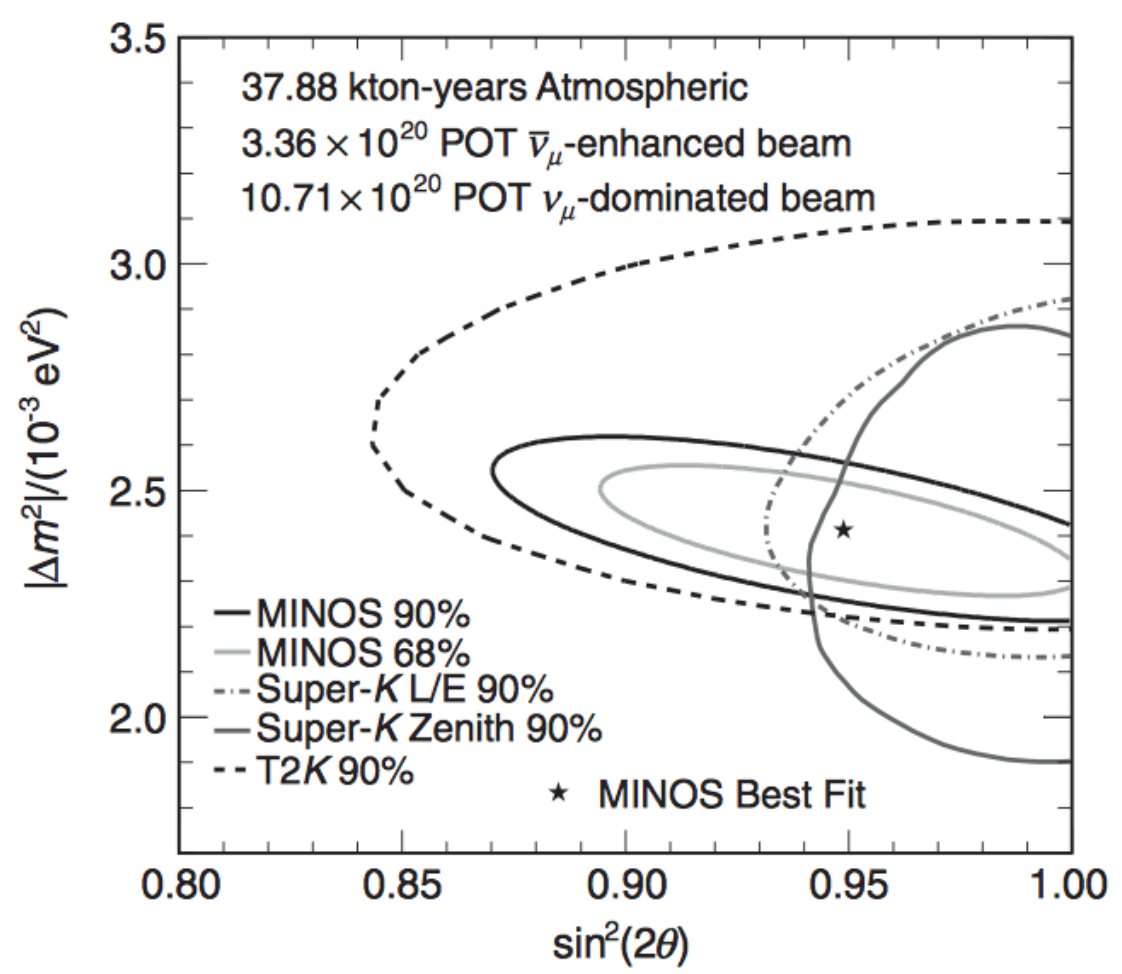

Figure 1.7: The 90\% C.L contour for $\Delta m^{2}$ vs $\sin ^{2}(2 \theta)$ from MINOS. This uses the full set of MINOS data which includes $v_{\mu}$ mode, $\bar{v}_{\mu}$ mode and 37.88 kiloton-year atmospheric data. The contour obtained from the Super-K $L / E$ analysis and T2K 90\% contour are shown [43]. 


\subsubsection{Reactor Neutrino Oscillation}

Reactor neutrino oscillation studies plays a crucial role in the development of the standard three flavour oscillation frame work. The experiments measure the disappearance of the $\bar{v}_{e}$, the disappearance probability is given by the Eqn. (1.27). The CHOOZ, situated in France, is one of the reactor experiment which study neutrino oscillations. This experiment studies the neutrinos coming from the CHOOZ nuclear plant having a thermal energy of $4.25 \mathrm{GW}$. The detector is $1 \mathrm{~km}$ away from the reactor and uses a 5 ton of liquid scintillator doped with Gd (for neutron capture) as the detecting material. This experiment did not observe any $\bar{v}_{e}$ disappearance and set an upper limit on the mixing parameter. The $\bar{v}_{e} \rightarrow \bar{v}_{\mu}$ oscillation excluded at $90 \%$ C.L for a value of $\Delta m^{2}>7 \times 10^{-4} \mathrm{eV}^{2}$ at maximal mixing, and $\sin ^{2}(2 \theta)>0.10$ for large $\Delta m^{2}$ [44]. Another experiment at Palo Verde in Arizona, also studied the neutrinos which are coming from three nuclear reactor, of which two are at a distance of 890 $\mathrm{m}$ and one at $750 \mathrm{~m}$ with a thermal power of $11.63 \mathrm{GeV}$. No evidence for neutrino disappearance was found. The $\bar{v}_{e} \rightarrow \bar{v}_{x}$ excluded at 90\% C.L for $\Delta m^{2}>1.1 \times 10^{-3} \mathrm{eV}^{2}$ at full mixing and $\sin ^{2}(2 \theta)>0.17$ [45].

The Double CHOOZ experiment, the successor of CHOOZ measured the neutrino oscillation using two detectors, one at $400 \mathrm{~m}$ and other is at $1.05 \mathrm{~km}$ away from the reactor. The experiment was designed to get the best sensitivity for the mixing angle $\theta_{13}$. The detector which is at $1.05 \mathrm{~km}$ away observed a total of 8249 electron antineutrino events with a $33.71 \mathrm{GW}$-ton year of exposure. The prediction with $\theta_{13}=0$, was 8937 events. The measured of value of $\theta_{13}$ from rate and spectral shape measurement is $\sin ^{2} 2 \theta_{13}=0.109 \pm 0.030$ (stat) \pm 0.025 (syst) [46]. The Daya Bay experiment in China has measured the value of $\theta_{13}$ precisely. The Daya Bay reactor experiment uses neutrinos coming out from 6 reactors, each with $2.9 \mathrm{GW}$ thermal power. The electron antineutrino energy produced by these reactor peaks at $3 \mathrm{GeV}$. The experiment used two Near Detector and one Far Detector, the Near Detectors are situated at $470 \mathrm{~m}$ and $576 \mathrm{~m}$, the Far Detector is situated at $1648 \mathrm{~m}$ away from the reactor respectively. The detecting material is Gadolinium doped liquid scintillator and the detector looks for the coincidence of the prompt scintillation from the $e^{+}$and delayed neutron captured on the Gadolinium. The best oscillation fit for the mixing angle $\theta_{13}$, is given by [47],

$$
\sin ^{2} 2 \theta_{13}=0.089 \pm 0.010 \text { (stat) } \pm 0.005 \text { (syst) }
$$


This is the most precise measurement of $\sin ^{2} 2 \theta_{13}$ to date with a precision of $12.6 \%$.

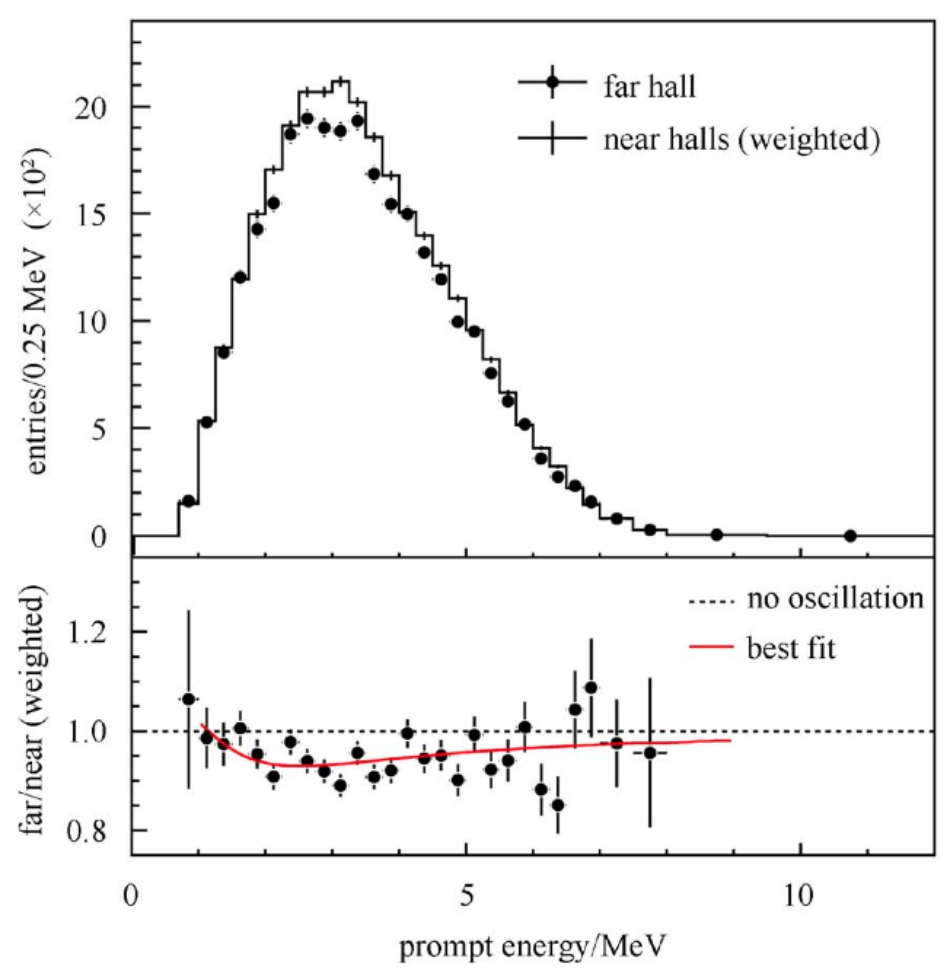

Figure 1.8: Top: The prompt energy spectrum of the far hall (sum of three ADs) compared with the no-oscillation prediction based on the measurements of the two near halls. Spectra were background subtracted. Uncertainties are statistical only. Bottom: The ratio of measured and predicted no-oscillation spectra. The solid curve is the expected ratio with oscillations, calculated as a function of neutrino energy assuming $\sin ^{2} 2 \theta_{13}=0.089$ obtained from the rate based analysis. The dashed line is the no-oscillation prediction. Plot is taken from [47].

A similar measurement has been confirmed by Reactor Experiment for Neutrino Oscillations (RENO). RENO is situated in Korea. The neutrinos are obtained from the 6 reactor at the Yonggwang Nuclear Power plant, having a thermal energy of $2.8 \mathrm{GW}_{t h}$. Neutrinos are detected by two identical detectors located at $294 \mathrm{~m}$ and $1383 \mathrm{~m}$ away from the neutrino 
source. By comparing the expected and observed event rate at the Far Detector the value of $\theta_{13}$ is obtained, the best fit is $\sin ^{2} 2 \theta_{13}=0.113 \pm$ 0.013 (stat) \pm 0.019 (syst) [48].

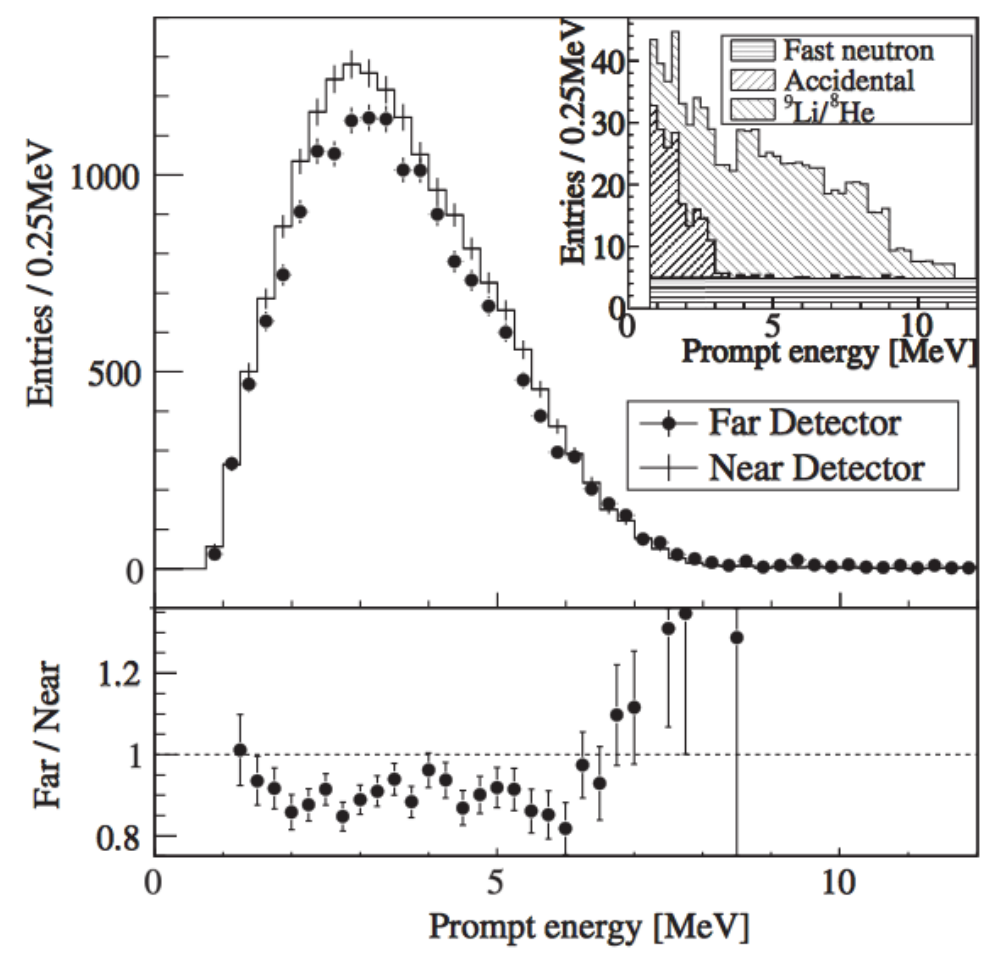

Figure 1.9: The observed prompt energy spectrum at Far Detector compared with the non-oscillation predictions from the measurements in the Near Detector. The backgrounds shown in the inset are subtracted for the far spectrum. The background fraction is 5.5\% (2.7\%) for Far (Near) detector. Errors are statistical uncertainties only. Bottom: The ratio of the measured spectrum of Far Detector to the non-oscillation prediction. Plot is taken from [48].

Long baseline neutrino oscillation experiments also look for the $v_{e}$ appearance and aim at measuring the value of $\theta_{13}$. The T2K experiment observed electron neutrino in muon neutrino beam. T2K at the Far Detector observed a total of $11 v_{e}$ events, with a background of $3.3 \pm 0.4$ (stat) 
events [49]. The best fit values for $\sin ^{2}\left(2 \theta_{13}\right)$ with the $68 \%$ C.L are,

$$
\begin{aligned}
& \sin ^{2} 2 \theta_{13}=0.088_{-0.039}^{+0.049} \text { (Normal Ordering) } \\
& \sin ^{2} 2 \theta_{13}=0.108_{-0.046}^{+0.059} \text { (Inverted Ordering) }
\end{aligned}
$$
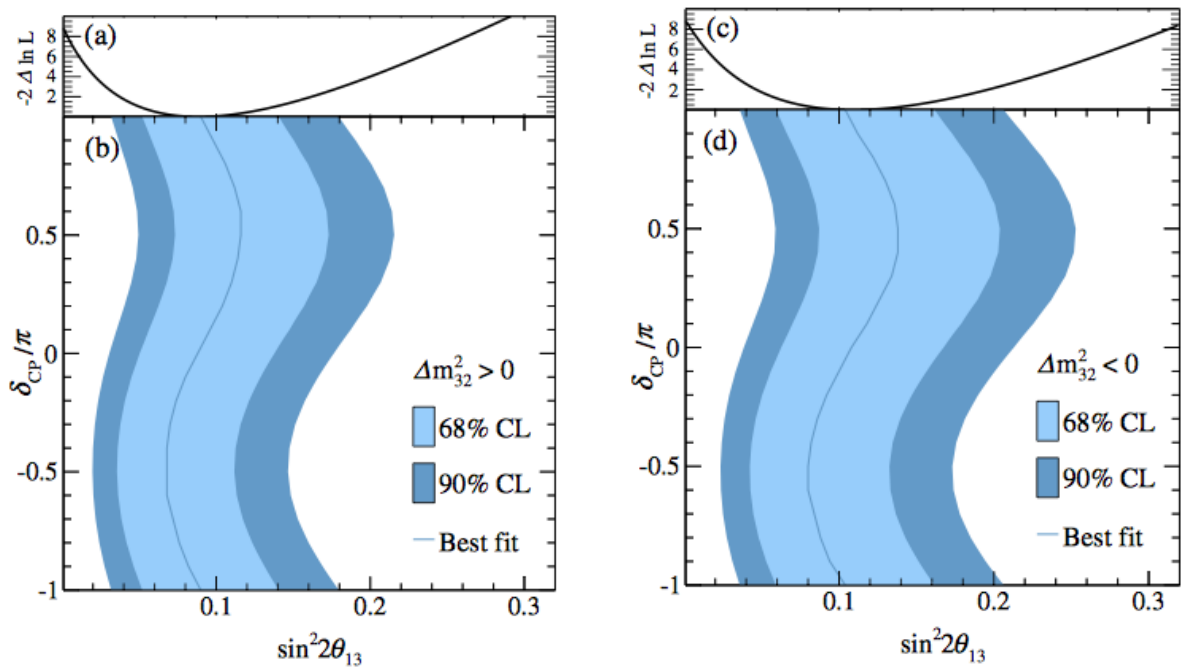

Figure 1.10: The $68 \%$ and $90 \%$ C.L for $\sin ^{2} 2 \theta_{13}$ scanned over values of $\delta_{C P}$. Left plot for normal hierarchy and right plot is inverted hierarchy. Plot is taken from [49].

A similar study has been done in MINOS. The NOvA experiment at Fermilab is designed to measure the $v_{e}$ appearance from a $v_{\mu}$ beam has recently published the first result. The experiment confirms the $v_{e}$ appearance signal at $3.3 \sigma$. The contours from MINOS and NOvA are shown in Fig. 1.11. 

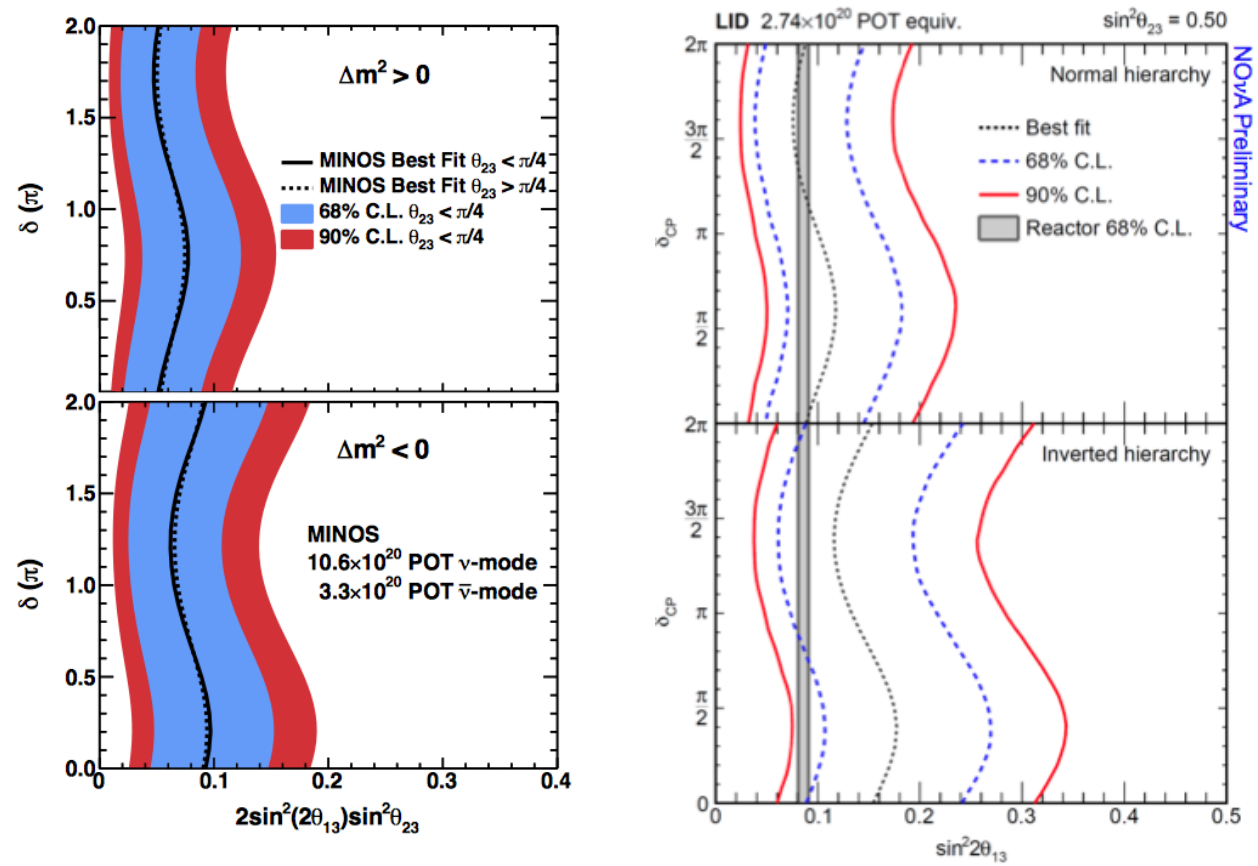

Figure 1.11: The $68 \%$ and $90 \%$ C.L for $\sin ^{2} 2 \theta_{13}$ values of $\delta_{C P}$ for MINOS (left) [50] and the latest results from NOvA, showing the $\sin ^{2} 2 \theta_{13}$ scanned over values of $\delta_{C P}$ for normal mass ordering and inverted mass ordering (right).

\subsection{Future Neutrino Programmes}

Neutrino physics has entered in the era of precision measurement. The upcoming neutrino programs will answer questions like, mass ordering of the neutrinos, whether neutrinos violate $\mathrm{CP}$, measure the octant of mixing angle $\theta_{23}$, do we have more than three neutrinos etc.

\subsubsection{Mass Ordering}

Neutrino oscillation experiments have not been able to resolve the sign of $\Delta m_{23}^{2}$ and $\Delta m_{31}^{2}$. Therefore, if $\Delta m_{23}^{2}$ is positive, i.e, $m_{3}>>m_{2}, m_{1}$ it is known as Normal Hierarchy $(\mathrm{NH})$. If the sign is negative, i.e, $m_{3}<<$ $m_{2}, m_{1}$ the scenario is called Inverted Hierarchy $(\mathrm{IH})$. It is very important to know the mass ordering of neutrinos as it gives hints to solve problem of whether neutrinos are its own antiparticle. 


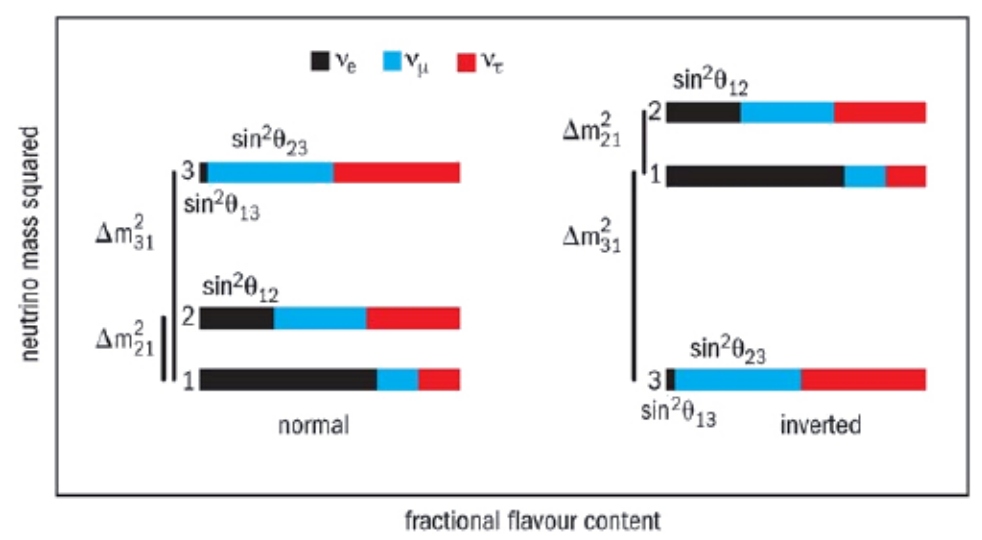

Figure 1.12: Two possible mass ordering for neutrino.

\subsubsection{CP violation}

A CP violation is a measure of a particle and its corresponding antiparticle behaves differently. Partner of a neutrino under CP transformation is an antineutrino. If CP-symmetry on neutrinos are violated, then it is expected that the probabilities of some phenomena between neutrinos and anti-neutrinos are different. There are many projects designed to investigate the difference of the neutrino oscillation probabilities between neutrinos and anti-neutrinos with intensive neutrino and antineutrino beam. $\mathrm{CP}$ violation is expressed by an phase $\delta$. It is the phase $\delta$ in PMNS matrix which leads to this $\mathrm{CP}$ violation. If we could measure difference in oscillation probability between neutrino and antineutrino, which would then will be a measure of CP-violation, i.e, $P\left(v_{\alpha} \rightarrow v_{\beta}\right) \neq P\left(\bar{v}_{\alpha} \rightarrow \bar{v}_{\beta}\right)$, for $\alpha \neq \beta$. The CP-violation can measure only in appearance experiment. Disappearance experiment is a $\mathrm{T}$ invariant process, therefore $\mathrm{CP}$ must be invariant.

\subsection{3 $\theta_{23}$ Octant}

The angle $\theta_{23}$ is a measure of admixture of mass state $\nu_{3}$. So far the measurement is consistent with the value of $\theta_{23}=45^{\circ}$. If it is exactly $45^{0}$, it says $\nu_{3}$ contains $v_{\mu}$ and $\nu_{\tau}$ equally. This is known as maximal mixing in neutrino physics. We can measure the mixing is maximal or non-maximal by the accurate measurement of the the $v_{\mu}$ disappearance. The difficulty 
is that, the leading order term in the probability depends on $\sin ^{2} 2 \theta_{23}$ and which is close to one, so the probability will be same for $2 \theta_{23} \gtrsim 90^{\circ}$ and $2 \theta_{23} \lesssim 90^{\circ}$.

The CP violation in the lepton sector will answer why we have matter dominated universe. All the future neutrino experiments will answer the most fundamental questions about the nature of neutrinos [51]. DUNE [52], JUNO [53], INO [54], LAGUNA [55], Hyper-K [56], CHIPS [57] are some of the future projects which will be trying to understand the nature of this elusive neutrinos. 



\section{2 \\ Sterile Neutrinos}

In the standard model of particle physics there are only three neutrinos $v_{e}, v_{\mu}$ and $v_{\tau}$. But there are hints for additional neutrino states with masses at the eV scale [58]. The LEP (Large Electron-Positron) experiment at CERN confirmed that there are only three active neutrinos coupled to the $\mathrm{Z}^{0}$ boson by measuring the decay width of the $\mathrm{Z}^{0}$ boson [59. This indicates that if there are additional neutrinos, since it do not have any coupling with the $\mathrm{Z}^{0}$ boson, hence do not participate in any standard model interaction, these neutrinos are called sterile neutrinos. A sterile neutrino is a neutral lepton with no ordinary weak interaction except those induced by mixing. Sterile neutrinos are present in many extensions of standard model. These neutrinos may play an important role in the leptogenesis in the early universe. As sterile neutrino have mixing with ordinary neutrinos, neutrino oscillation experiments are very good probe to search for it. Astrophysics experiment, like dark matter searches are also looking for this fourth type neutrino.

Sterile neutrinos can be incorporated into the oscillation formalism by adding additional neutrino states. In general, for $N$ neutrino mass eigenstates, the neutrino mixing is parametrized by an $N \times N$ unitary matrix, with $N(N-1) / 2$ mixing angles and $N(N-1)(N-2) / 2$ phases. The mixing angles are a measure of the flavour content in a particular mass eigenstate. The phases are a measure of the leptonic CP-violation, which may have played a role in the generation of the matter-antimatter asymmetry in the early universe [60]. A simple $3+1$ model, which includes three active neutrinos and one sterile neutrino, is studied in this thesis. 


\subsection{Experimental Anomalies in Neutrino Physics}

Neutrino oscillation studies at short baseline have seen anomalies which can be explained by additional neutrino state. These anomalies are observed at an $L / E \sim 1 \mathrm{~m} / \mathrm{MeV}$, where $L$ is the distance travelled by neutrino and $E$ is the energy of the neutrino. The LSND (Liquid Scintillator Neutrino Detector) at Los Alamos Laboratory reported such an anomaly in the data [61]. The MiniBooNE (Mini Booster Neutrino Experiment) at Fermilab also reported similar results in the $\bar{v}_{\mu} \rightarrow \bar{v}_{\mathrm{e}}$ oscillation studies for a similar value of $L / E[62]$. The other anomaly includes, the reactor anomaly [63], radioactive anomalies in GALLEX (Gallium Experiment) [35] and the anomalies seen in the SAGE (Soviet-American Gallium Experiment), solar neutrino experiment [34].

\subsubsection{The LSND Anomaly}

The Liquid Scintillator Neutrino Detector (LSND) is a neutrino detector designed to study the neutrinos produced from the Los Alamos Meson Physics Facility (LAMPF) at Los Alamos Laboratory. The experiment studied $\bar{v}_{\mathrm{e}} \rightarrow \bar{v}_{\mu}$ oscillation and observed an excess of antineutrino events at a significance of $3.8 \sigma$ level above the expected background. This data was interpreted as two-flavour oscillation with very small mixing angle $\sin ^{2} 2 \theta \sim 0.003$ and large $\Delta m^{2} \sim 1 \mathrm{eV}^{2}$. LSND found an excess of $87.9 \pm$ $22.4 \pm 6.0 \bar{v}_{\mathrm{e}}$ events above a background of $30.0 \pm 6.0$ events. 

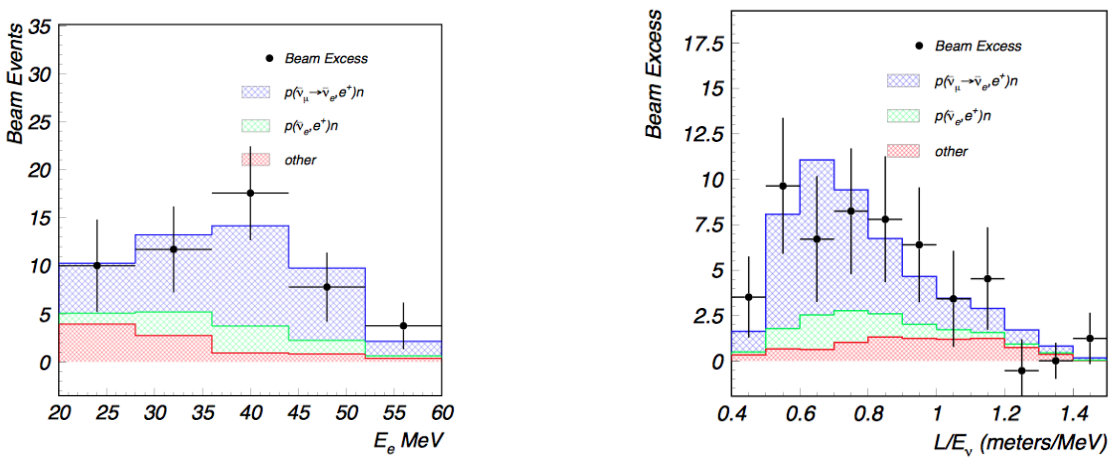

Figure 2.1: The observed excess in $v_{\mathrm{e}}$ events in LSND as function of energy (left) and as a function of $L / E$ (right). Plots taken from [58.

This excess is interpreted as neutrino oscillation with $\Delta m^{2} \sim 1 \mathrm{eV}^{2}$ and $\sin ^{2} 2 \theta_{\mu e} \sim 0.003$, which does not fit into the standard three-flavour neutrino oscillation picture, where the two independent mass squared differences are orders of magnitude different than the $1 \mathrm{eV}^{2}$. This allows for additional neutrinos states to exist with a higher $\Delta m^{2}$ value than the standard three neutrino scenario.
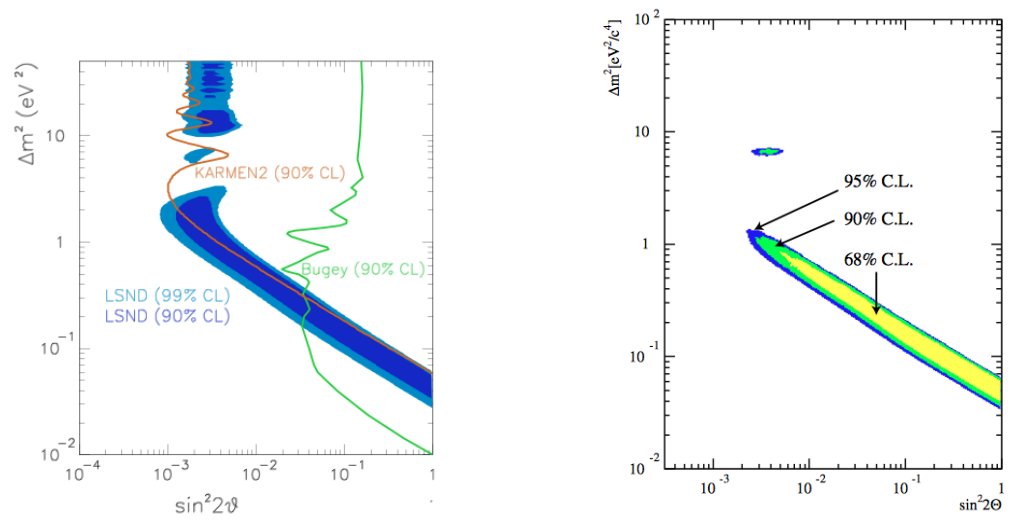

Figure 2.2: The fit results in terms of parameters space $\left(\Delta m^{2}\right.$ and $\left.\sin ^{2} 2 \theta\right)$. The fit includes both $\bar{v}_{\mathrm{e}} \rightarrow \bar{v}_{\mu}$ and $\nu_{\mathrm{e}} \rightarrow v_{\mu}$ oscillations. The $90 \%$ Confidence Limit (90\%C.L) from reactor Bugey experiment (green) and KARMEN2 (brown) are also shown (left). Combined analysis of KARMEN and LSND gives the allowed region (right). The figure taken from [64]. 


\subsubsection{The MiniBooNE Experiment}

The MiniBooNE [65] at Fermilab was built to test the LSND anomaly. The MiniBooNE detector is a spherical tank of diameter $12.2 \mathrm{~m}$ filled with 800 tons of mineral oil and use neutrinos produced by the Fermilab booster neutrino facility. The experiment was designed at the same $L / E$ $(\mathrm{km} / \mathrm{MeV})$ as that of the LSND. MiniBooNE looked for the $\bar{v}_{\mu} \rightarrow \bar{v}_{\mathrm{e}}$ and $v_{\mu} \rightarrow v_{e}$ oscillation by selecting the charged current quasi elastic (CCQE) event $v_{\mathrm{e}}+\mathrm{C} \rightarrow \mathrm{e}^{-}+\mathrm{X}$. The flux prediction of different neutrino components at MiniBooNE detector is shown in Fig. 2.3 .

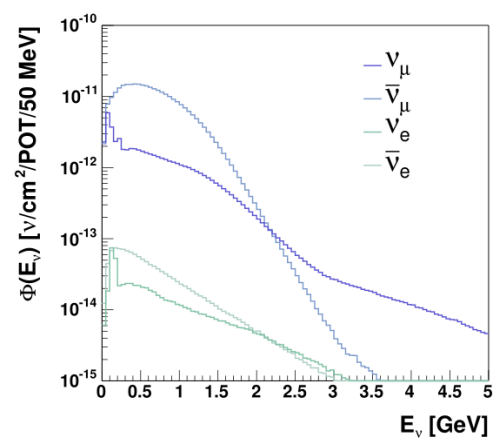

(a)

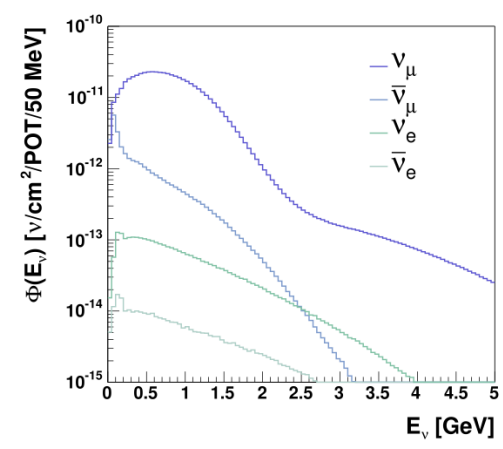

(b)

Figure 2.3: The flux prediction for different neutrino component at the MiniBooNE detector, for neutrino mode (right) and antineutrino mode (left) [64].

The reconstructed neutrino energy spectra with different background components is shown in Fig. 3.2. While data and Monte Carlo (MC) are in good agreement for neutrino energy, $E_{Q E}>475 \mathrm{MeV}$ and an excess of neutrino events is observed below $475 \mathrm{MeV}$. The MiniBooNE sample is split up into three regions, $200-300 \mathrm{MeV}$, 300-475 MeV and 475-1250 MeV respectively and correspondingly the total expected background in these regions is $186.8 \pm 26.0$ events, $228.3 \pm 24.5$ events, and $385.9 \pm 35.7$ events. For $v_{\mu} \rightarrow v_{\mathrm{e}}$ oscillation at the LSND best fit $\left(\Delta m^{2}=1.2 \mathrm{eV}^{2}, \sin ^{2} 2 \theta=0.003\right.$ ), the expected number of $v_{\mathrm{e}}$ CCQE events are 7,37 and 135 respectively. The final analysis found an excess of $128.8 \pm 20.4 \pm 38.3$ events in the energy range $200 \mathrm{MeV}<E^{Q E}<475 \mathrm{MeV}$ and for the full energy 
range $\left(200 \mathrm{MeV}<E^{Q E}<1250 \mathrm{MeV}\right)$ there are $151.0 \pm 28.3 \pm 50.7$ excess events [64].

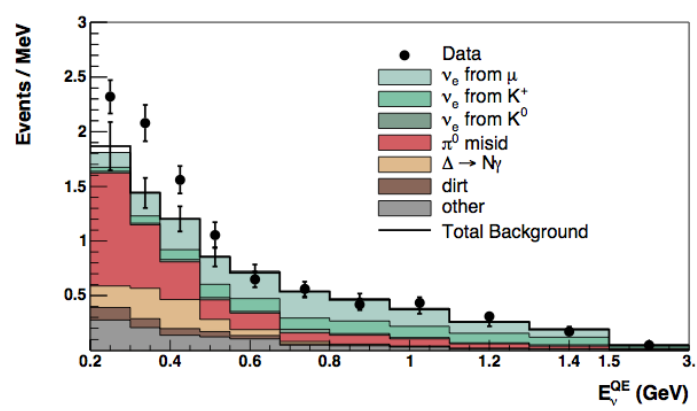

(a)

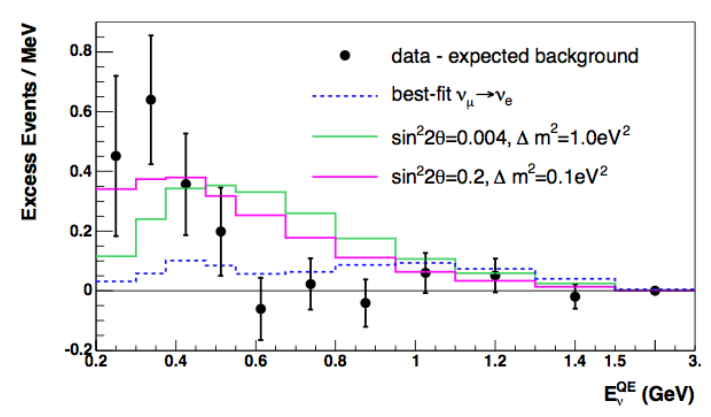

(b)

Figure 2.4: The reconstructed energy spectra for neutrino in MiniBooNE detector with different backgrounds (top). The excess of neutrino events seen in the MiniBooNE as function of $E^{Q E}$ (bottom). Figure taken from 64].

MiniBooNE also observed an excess of events in the $\bar{v}_{\mu}$ mode data. In the $\bar{v}_{\mu}$ it is found that there are $54.9 \pm 17.4 \pm 16.3$ excess events in the $200 \mathrm{MeV}<E^{Q E}<1250 \mathrm{MeV}$ energy range. The allowed region of parameters space obtained from the antineutrino fit results are shown in Fig. 2.5, which is consistent with LSND result. The fit for this study was performed by taking into account for the bin-to-bin correlations in the uncertainties, which includes flux, cross section and detector systematics. 


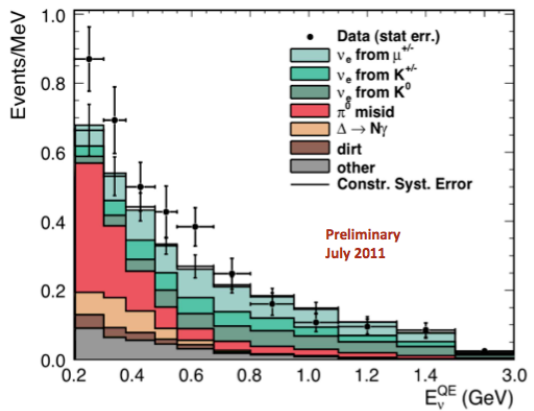

(a)

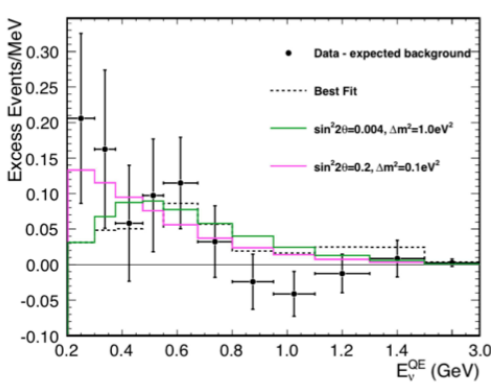

(b)

Figure 2.5: The reconstructed energy spectra for antineutrino in MiniBooNE with different backgrounds (right). The excess of antineutrino events seen in the MiniBooNE as function of $E^{Q E}$ (left). Figure taken from [64].

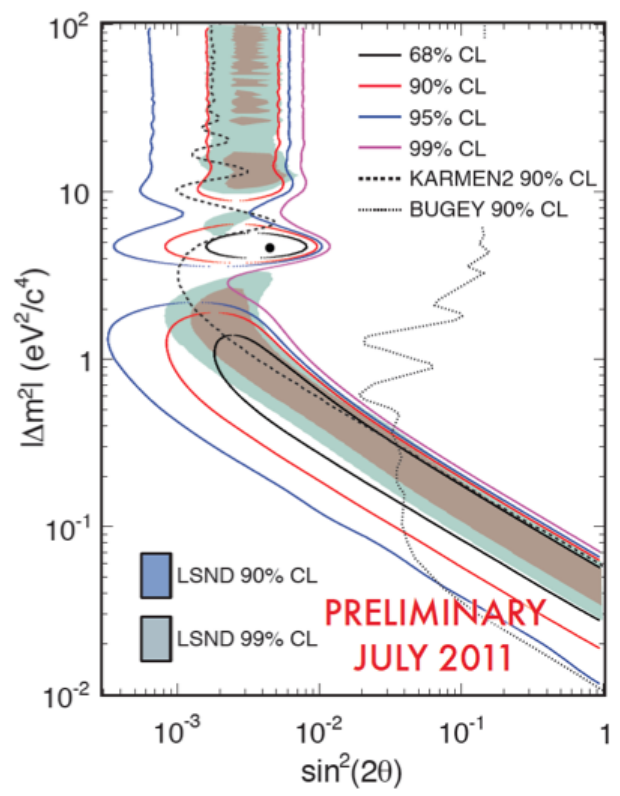

Figure 2.6: The allowed region of parameter space obtained from the MiniBooNE antineutrino fit result, which is consistent with LSND signal [64]. 


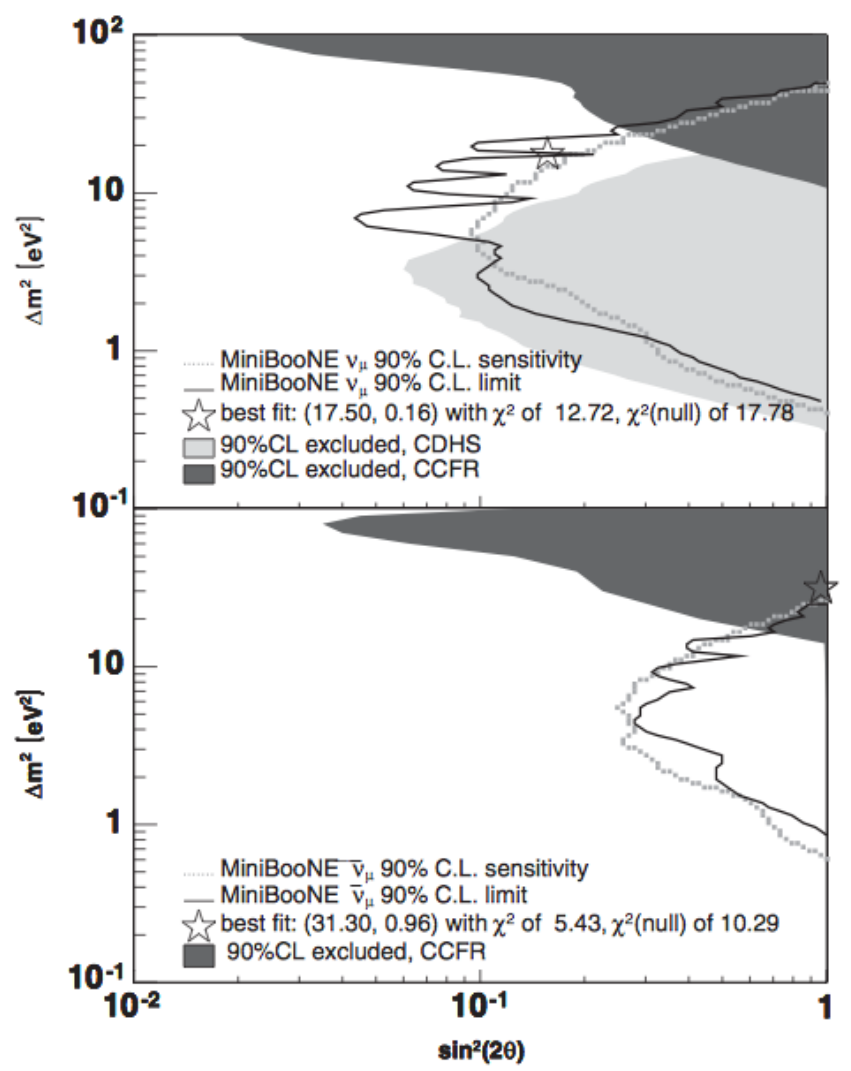

Figure 2.7: The top plot shows the sensitivity (dashed line) and limit (solid line) for $90 \%$ C.L. for neutrino disappearance in MiniBooNE. Previous limits by CCFR (Chicago - Columbia - Fermilab - Rochester) in dark grey and CDHS (CERN - Dortmund - Heidelberg - Saclay experiment) in light grey are also shown. The bottom plot shows the antineutrino disappearance limit [66].

\subsubsection{The Short Baseline (SBL) Reactor Anomalies}

Reactor neutrino anomalies came from the re-analysis of old neutrino data using the newly evaluated $\bar{v}_{\mathrm{e}}$ flux [67]. The newly evaluated flux is $3.5 \%$ larger than the flux previously used. For neutrinos traveling a distance less than $100 \mathrm{~m}$, this leads to a change in the ratio of observed to expected events from $0.976 \pm 0.024$ to $0.943 \pm 0.023$ which is a deviation from unity at $98.6 \%$ Confidence Limit (C.L). 

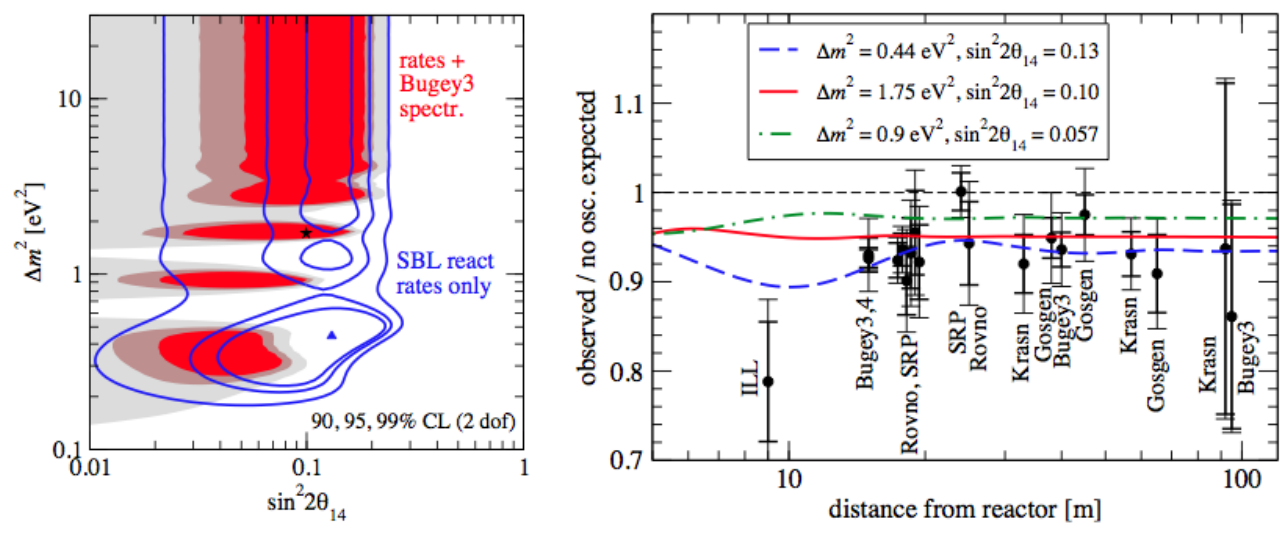

Figure 2.8: The allowed regions of oscillation parameters from the SBL data with $3+1$ model (left). Ratio of observed to expected number of events, together with prediction for different $\Delta m^{2}$ and $\sin ^{2} 2 \theta_{14}$ values (right). The figure is taken from [68].

\subsubsection{The Gallium Anomaly}

The GALLEX [35] and SAGE (Soviet-American Gallium Experiment) [34] solar neutrino detectors were used to perform the radioactive source experiment, these detectors basically look for the electron neutrino $\left(v_{\mathrm{e}}\right)$ produced by the intense radioactive source of $\mathrm{Cr}$ and $\mathrm{Ar}$ placed inside the detector and these radioactive nuclei emits $v_{\mathrm{e}}$ through electron capture. The neutrino energy threshold for this reaction is $0.233 \mathrm{MeV}$. The number of events observed is less than expected at a $2.8 \sigma$ level. 


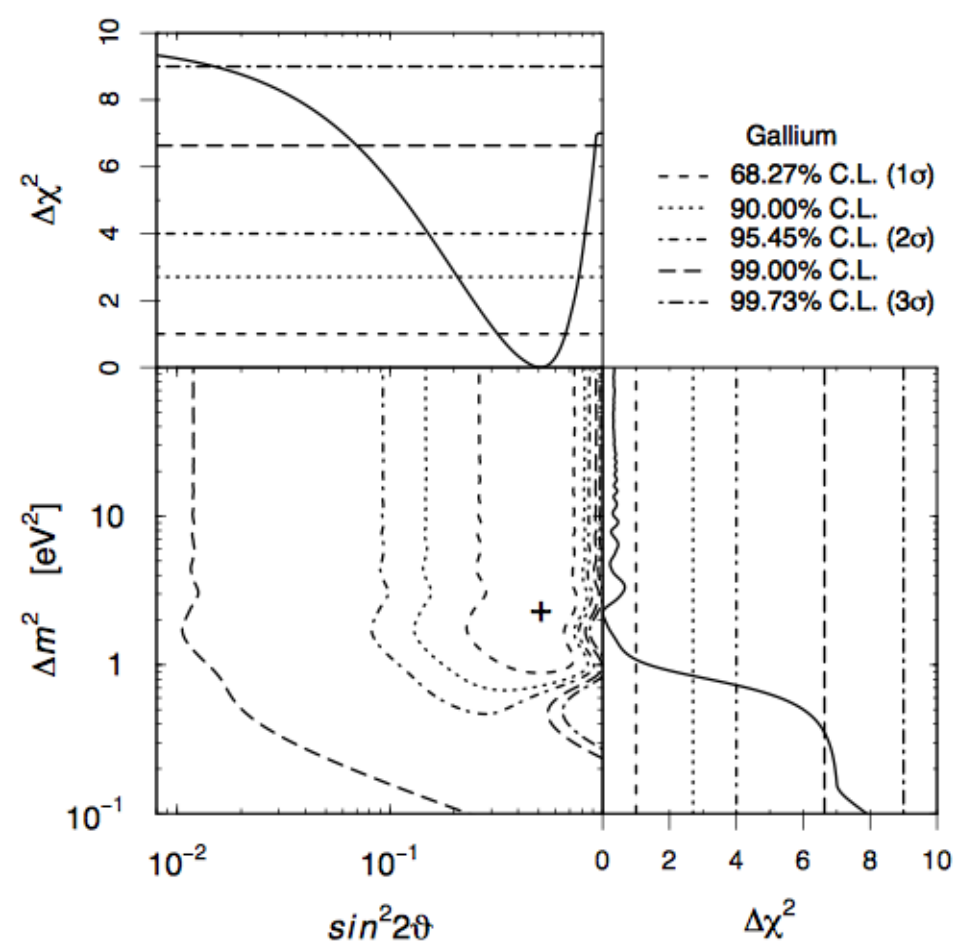

Figure 2.9: The allowed region in the $\Delta m^{2}-\sin ^{2} 2 \theta$ plane obtained from the combined fit of the results of the two GALLEX Cr radioactive source experiments, and the SAGE $\mathrm{Cr}$ and Ar radioactive source experiments [64].

\subsection{Four-Flavour Oscillation Model (3+1 Model)}

The addition of a fourth neutrino mass and flavour state requires the PMNS mixing matrix to be generalized to four dimensions,

$$
U=\left(\begin{array}{cccc}
U_{e 1} & U_{e 2} & U_{e 3} & U_{e 4} \\
U_{\mu 1} & U_{\mu 2} & U_{\mu 3} & U_{\mu 4} \\
U_{\tau 1} & U_{\tau 2} & U_{\tau 3} & U_{\tau 4} \\
U_{s 1} & U_{s 2} & U_{s 3} & U_{s 4}
\end{array}\right)
$$

In the standard formalism [69], probability of neutrino with one flavour 
transforming to another flavour can be written as,

$$
\begin{aligned}
P\left(v_{\alpha} \rightarrow v_{\beta}\right) & =\delta_{\alpha \beta}-4 \sum_{j>i} \Re\left(U_{\alpha j}^{*} U_{\beta j} U \alpha i U_{\beta i}^{*}\right) \sin ^{2} \Delta_{i j} \\
& +2 \sum_{j>1} \operatorname{Im}\left(U_{\alpha j}^{*} U_{\beta j} U_{\alpha i} U_{\beta i}^{*}\right) \sin \left(2 \Delta_{i j}\right),
\end{aligned}
$$

where $\Delta_{i j}=\left(m_{i}^{2}-m_{j}^{2}\right) L /(4 E), \mathrm{L}$ is the distance travelled by the neutrino, $\Re$, Im are real and imaginary part and $\mathrm{E}$ is the energy of the neutrino. The formula can be simplified on the basis of some approximation, oscillation driven by $\Delta m_{21}^{2}$ is too small and can be neglected, so the approximation $\sin ^{2} \Delta_{21}=\sin \left(2 \Delta_{21}\right)=0$ is valid. This leads to equivalent approximation $\Delta_{42}=\Delta_{41}, \Delta_{32}=\Delta_{31}$ and leads to the following expression for the survival probability of $v_{\mu}$ or $\left(\bar{v}_{\mu}\right)$,

$$
\begin{aligned}
P\left(v_{\mu} \rightarrow v_{\mu}\right) & =1-4\left|U_{\mu 3}\right|^{2}\left(1-\left|U_{\mu 3}\right|^{2}-\left|U_{\mu 4}\right|^{2}\right) \sin ^{2} \Delta_{31} \\
& +4\left|U_{\mu 4}\right|^{2}\left|U_{\mu 3}\right|^{2} \sin ^{2} \Delta_{43} \\
& +4\left|U_{\mu 4}\right|^{2}\left(1-\left|U_{\mu 3}\right|^{2}-\left|U_{\mu 4}\right|^{2}\right) \sin ^{2} \Delta_{41},
\end{aligned}
$$

where,

$$
\begin{gathered}
U_{\mu 3}=-\sin \theta_{14} \sin \theta_{13} e^{-i \delta_{1}} \sin \theta_{24} e^{-i \delta_{2}}+\cos \theta_{13} \sin \theta_{23} \sin \theta_{24}, \\
U_{\mu 4}=-\cos \theta_{14} \sin \theta_{24} e^{-i \delta_{2}} .
\end{gathered}
$$

Measurements from short-baseline experiments suggest that $\theta_{14}$ is small [64, even if the signal suggested by the reactor antineutrino anomalies are positive, it also suggest a small value of $\theta_{14}$ hence the approximation $\sin \theta_{24} \cos \theta_{14} \approx \sin \theta_{24}$ can be made. Then matrix element in the Eq. (5.3) simplify to

$$
U_{\mu 3}=\sin \theta_{23} \cos \theta_{24}, \quad U_{\mu 4}=\sin \theta_{24} e^{-i \delta_{2}} .
$$

In the region where, $\Delta m_{43}^{2}>10^{-1} \mathrm{eV}^{2}$, with in the resolution of the MINOS Far Detector $\sin ^{2} \Delta_{41}$ and $\sin ^{2} \Delta_{43}$ average to $\frac{1}{2}$. Therefore at Far Detector, the survival probability can be written as

$$
\begin{aligned}
P_{v_{\mu} \rightarrow v_{\mu}} & =1-4\left\{\left|U_{\mu 3}\right|^{2}\left(1-\left|U_{\mu 3}\right|^{2}-\left|U_{\mu 4}\right|^{2}\right) \sin ^{2} \Delta_{31}+\frac{\left|U_{\mu 4}\right|^{2}}{2}\left(1-\left|U_{\mu 4}\right|^{2}\right)\right\} \\
& =1-\sin ^{2}\left(2 \theta_{23}\right) \cos ^{4} \theta_{24} \sin ^{2} \Delta_{31}-\frac{1}{2} \sin ^{2} 2 \theta_{24}
\end{aligned}
$$




\subsection{Sterile Neutrinos in MINOS}

MINOS also calculated the mixing between active and sterile neutrinos by measuring the Neutral Current (NC) events rate [70]. Since NC cross section is same for all the three active neutrinos, the total NC event rate are unaffected by the standard three-flavour oscillation. The mixing between sterile and active neutrino cause a energy dependent depletion in the Far Detector.

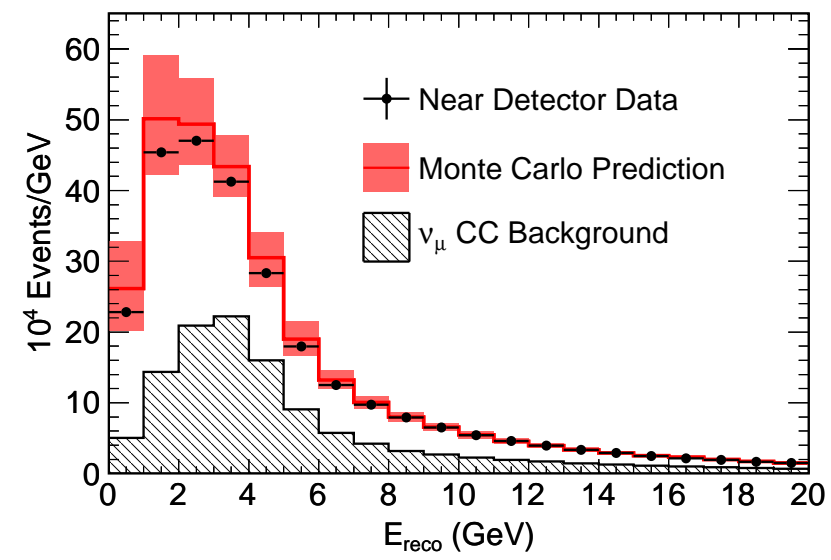

Figure 2.10: The plot shows the reconstructed NC selected events in MINOS Near Detector data and compared with MC simulation, the band shows $1 \sigma$ error in the MC, the CC background is also shown in the back histogram. Figure taken from [71]. 


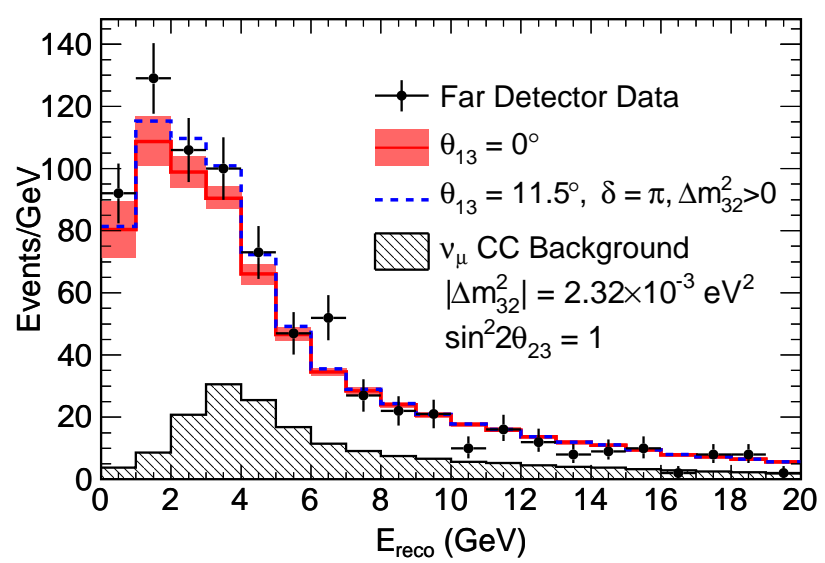

Figure 2.11: The plot shows the reconstructed NC selected events in MINOS Far Detector data and compared with MC simulation, the band shows $1 \sigma$ error in the MC, also the CC background is also shown in the back histogram. Figure is taken from [71].

The agreement between the observed and predicted NC spectra is quantified using a statistic $R$,

$$
R=\frac{N D_{\{d a t a\}}-B_{C C}}{S_{N C}},
$$

where, $N D_{\{\text {data\} }}$ is the observed event rate in the given energy range, $B_{\mathrm{CC}}$ is the extrapolated CC interaction from all flavours and $S_{\mathrm{NC}}$ is the extrapolated number of NC interactions. 


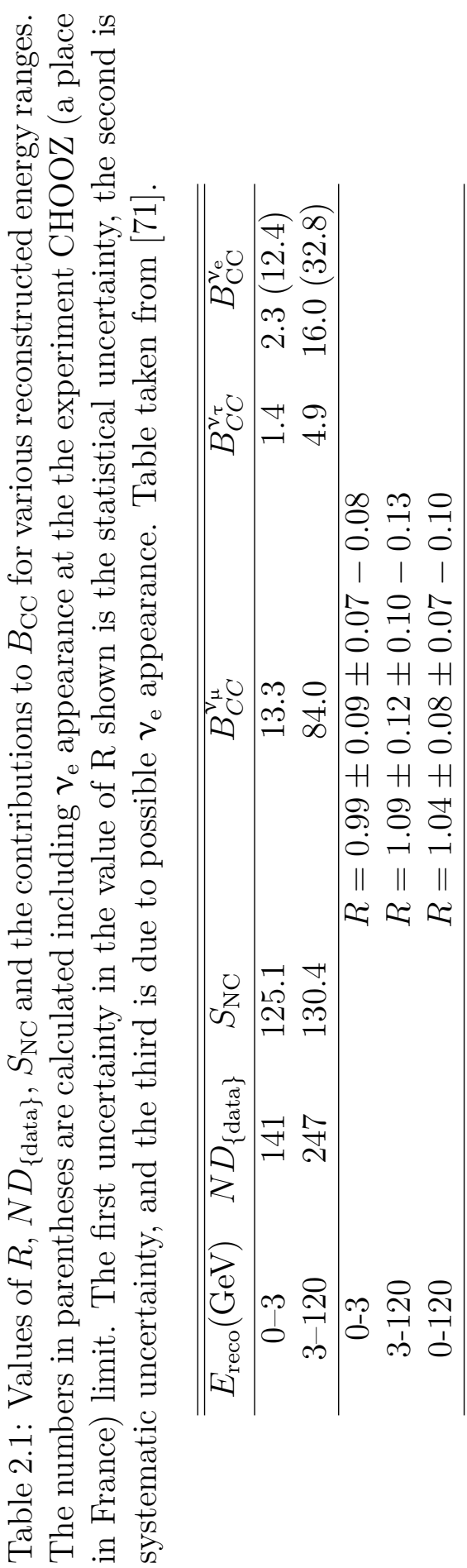




\subsection{Context of the Thesis}

This thesis includes the search result for sterile antineutrinos using $3.36 \times 10^{20}$ Protons On Target (POT). The MINOS experiment has collected data in antineutrino mode between the year 2009-2011. The excess seen by MiniBooNE and LSND in the antineutrino mode data can be compared directly with MINOS $\bar{v}_{\mu}$ disappearance results after combining with the $\bar{v}_{\mathrm{e}}$ disappearance data. The results in this thesis include the sterile neutrino search using the charged current selected $\bar{v}_{\mu}$ events. A limit has been set on mixing angle $\theta_{24}$ using the CC disappearance of the $\bar{v}_{\mu}$. This thesis also describes a combination technique of MINOS results with the reactor neutrino experiment at BUGEY (a place in France) which looks for the $\bar{v}_{\mathrm{e}}$ disappearance. The combined results gives a limit which probes the appearance channel angle, so that the result can be directly compared to the LSND and MiniBooNE signal. 


\section{The NuMI Beam and the MINOS Experiment}

MINOS (Main Injector Neutrino Oscillation Search) is a long baseline neutrino experiment, designed to study neutrino oscillation in the atmospheric sector. MINOS uses $v_{\mu} / \bar{v}_{\mu}$ produced at the NuMI beamline at Fermilab and directed toward two detectors, the Near Detector (ND), located $1.04 \mathrm{~km}$ away from the NuMI beam target and Far Detector (FD) located in the Soudan Mine at Minnesota, $735 \mathrm{~km}$ away from the beam target. A schematic representation of the experiment is shown in Fig. 3.1. The Near Detector measures the neutrino energy spectrum and the beam composition and Far Detector measures the oscillated neutrino energy spectrum. By comparing the two energy spectra, neutrino oscillation parameter can be precisely measured.
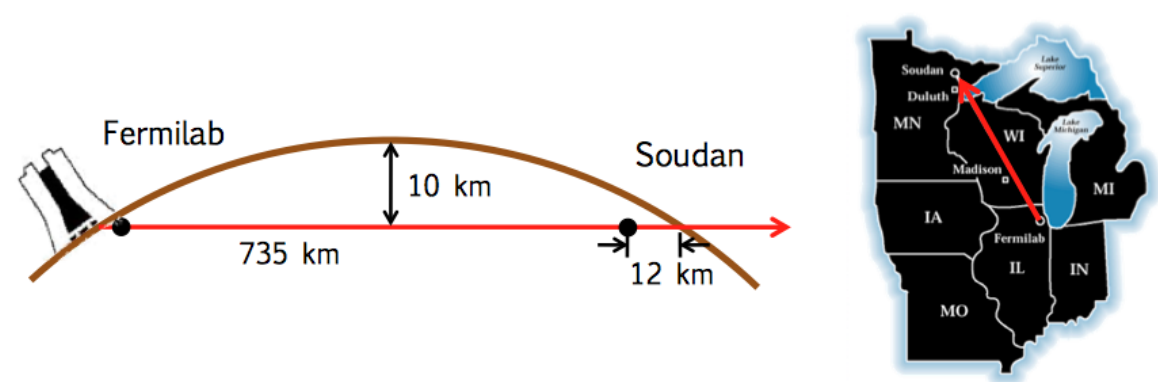

Figure 3.1: Schematic representation of the MINOS experiment. The Near Detector (ND) is at Fermilab and the Far Detector (FD) is at Soudan, Minnesota [69]. 


\subsection{MINOS Physics}

The MINOS measures the mixing parameter, $\Delta m_{23}^{2}$ with an accuracy of $10 \%$ and put a limit on the mixing angle $\sin ^{2} 2 \theta_{23}$. The measured Near Detector spectrum can be used to predict the Far Detector energy spectrum in the absence of oscillation and then compared with the observed Far Detector energy spectrum see (Figure. 3.2). A clear deficit of number of events is observed at the Far Detector which can be interpreted due to the neutrino oscillation.

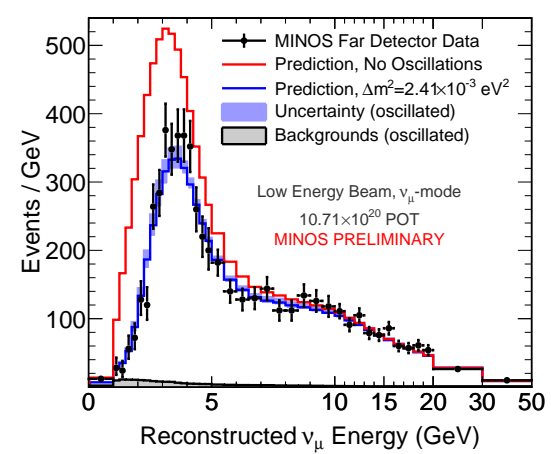

(a)

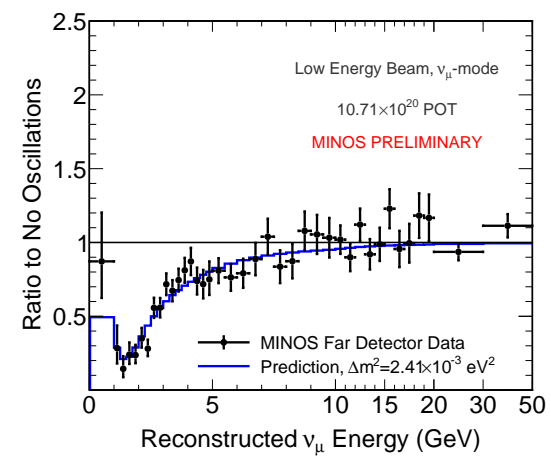

(b)

Figure 3.2: (a) The results of the MINOS disappearance with $v_{\mu}$ beam data, comparing the FD data (black) to the prediction without oscillation (red) and the prediction with oscillation (blue). Data agrees well with the oscillation prediction. (b) The ratio to the no oscillation, a clear oscillation dip is visible, the best fit oscillation are shown in blue.

The ratio of data to no oscillation can be translated into the survival probably of $v_{\mu}, P\left(v_{\mu} \rightarrow v_{\mu}\right)$. A characteristic dip is seen around $1.5 \mathrm{GeV}$. The magnitude of the dip is governed by $\sin ^{2} 2 \theta_{23}$, whilst the energy at the dip is a measure of $\Delta m_{23}^{2}$. 


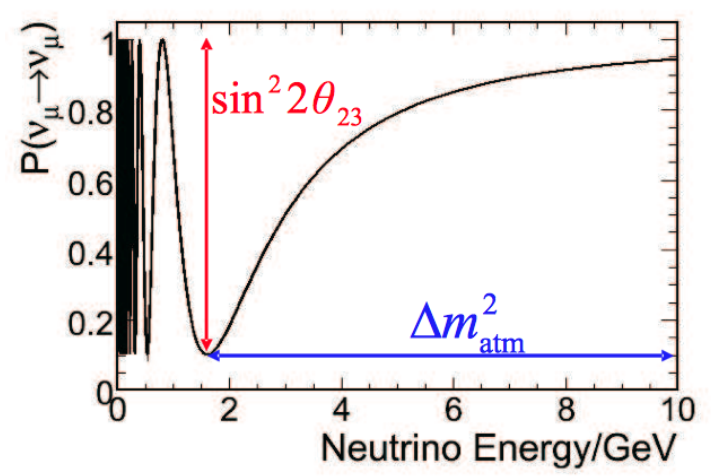

Figure 3.3: The survival probability of a muon neutrino in a two-flavour model as a function of neutrino energy, the position of the dip is the measure of mass squared splitting, $\Delta m_{\text {atm }}^{2}$ and depth of the dip is the measure of mixing angle, $\sin ^{2} 2 \theta$. Figure is taken from [69].

MINOS also measured the mixing angle $\theta_{13}$ [72], by studying the appearance of $v_{\mathrm{e}}$ at the Far Detector. The experiment studied many other interesting physics which includes, the non-standard interaction and the constrained flavour changing neutrino interaction [73] and the propagation speed of neutrinos as they travel from Fermilab to Soudan. The result of the measurement is consistent with theory [74. CPT violation was tested in MINOS by comparing the probability difference of neutrino and antineutrino oscillation in the disappearance channel [23].

\subsection{The NuMI Beam}

The neutrinos for the MINOS experiment are provided by the NuMI (Neutrino at Main Injector) facility at Fermilab. A graphical representation of NuMI facility is shown in Fig. 3.4. Here $120 \mathrm{GeV}$ protons are extracted from the Main Injector (MI) in a pulse form (spill) which last for $10 \mu \mathrm{s}$ in every 2.2 seconds. These protons collide with a water cooled segmented graphite target (47 segments with a thickness of 1.9 nuclear interaction lengths) and produces hadrons. Before the beam reaches the target it passes through the a graphite baffle $(150 \mathrm{~cm}$ long and $11 \mathrm{~mm}$ radius), which helps to reduce the intensity of badly focused protons and protects the downstream components. 


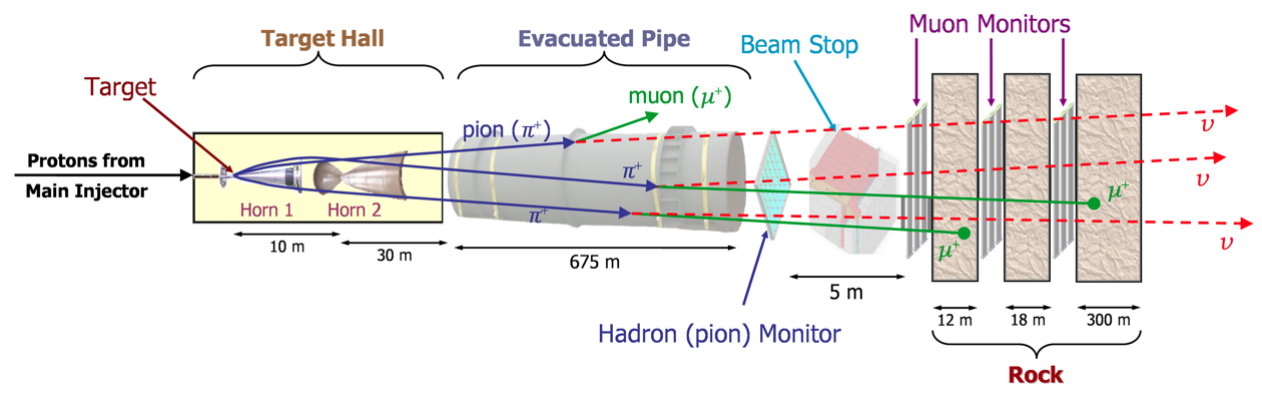

Figure 3.4: The graphical representation of the NuMI beam and neutrino production. The figure is taken from [23].

The hadrons produced in the target are focused using two magnetic horns, placed just behind the target. The hadrons are mostly pions and kaons, which then decay predominantly into muons and neutrinos in a $675 \mathrm{~m}$ long decay pipe. The decay of $\pi^{+} \rightarrow \mu^{+}+v_{\mu}$ and $\mathrm{K}^{+} \rightarrow \mu^{+}+v_{\mu}$ produces the $v_{\mu}$ beam, which will also have some small fraction of $\bar{v}_{\mu}$ coming from the $\pi^{-}$and $\mathrm{K}^{-}$produced in the target and a small fraction of $v_{\mathrm{e}} / \bar{v}_{\mathrm{e}}$ due to the subdominant decay mode of $\mathrm{K}^{+}, \mathrm{K}^{0}$ and decay of the tertiary muons. A hadron monitor, which is a $5 \mathrm{~m}$ long absorber placed after the decay pipe profiles the residual hadrons in the beam and most of them stops inside. A muon monitor, profiles the residual muons placed after the hadron monitor completely stops all the remaining muons such that we are left with pure neutrino beam.

The neutrino energy is tunable by changing the relative positions of target and horns as shown in Fig. 3.5. In MINOS, for getting the best measure of oscillation parameters, the beam is optimized to run in Low Energy configuration (LE). It has been found that a increased neutrino flux is obtained when target is $10 \mathrm{~cm}$ upstream and running the horns at a current of $185 \mathrm{kA}$. 

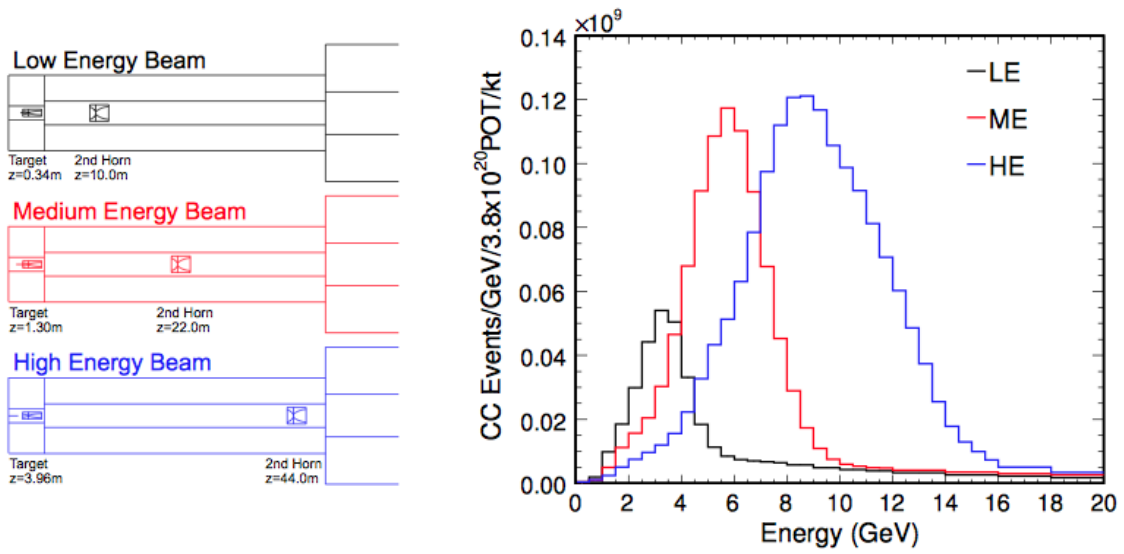

Figure 3.5: Configurations of the NuMI beam for the different neutrino energy spectrum. The relative positions of horns and target for the 3 different configurations, Low (LE), Medium (ME) and High (HE) Energy [69].

In the $v_{\mu}$ mode running the beam consists of $91.7 \% v_{\mu}, 7 \% \bar{v}_{\mu}$ and $1.3 \%\left(v_{\mathrm{e}}+\bar{v}_{\mathrm{e}}\right)$ while $\bar{v}_{\mu}$ mode the beam consists of $39.9 \% \bar{v}_{\mu}, 58.1 \% v_{\mu}$ and $2 \%\left(v_{\mathrm{e}}+\bar{v}_{\mathrm{e}}\right)$ [75]. The simulated $\boldsymbol{v}_{\mu}$ and $\bar{v}_{\mu}$ energy spectra at ND are shown in Fig. 3.6

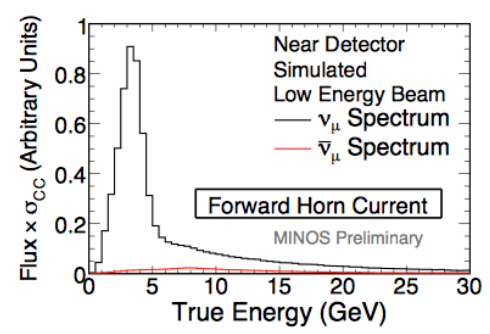

(a)

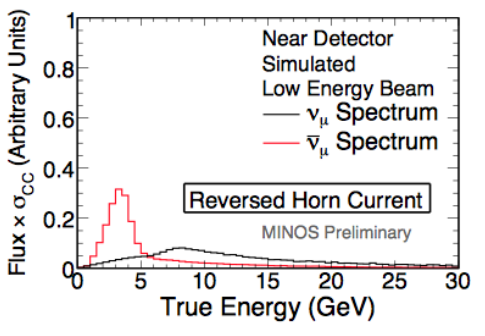

(b)

Figure 3.6: The $v_{\mu}$ and $\bar{v}_{\mu}$ at the Near Detector (a) For $v_{\mu}$ mode running, (b) $\bar{v}_{\mu}$ mode running.

\subsubsection{The Data Collection}

MINOS has started taking beam data through the years 2005-2012. In the seven years of running, MINOS has collected a data sample corresponding to $15.6 \times 10^{20}$ Protons-On-Target $(\mathrm{POT})$. This includes $10.56 \times$ 
$10^{20}$ POT of neutrino mode data $\left(v_{\mu}\right), 0.15 \times 10^{20}$ POT were collected in pseudo high energy (pHE), where the neutrino energy is around $10 \mathrm{GeV}$ and $3.36 \times 10^{20}$ POT of antineutrino mode data $\left(\bar{v}_{\mu}\right)$. This thesis uses the $3.36 \times 10^{20}$ POT of antineutrino mode data. Fig. 3.7 shows the details of the data collection and Table 3.1 summarizes the details of each run period.

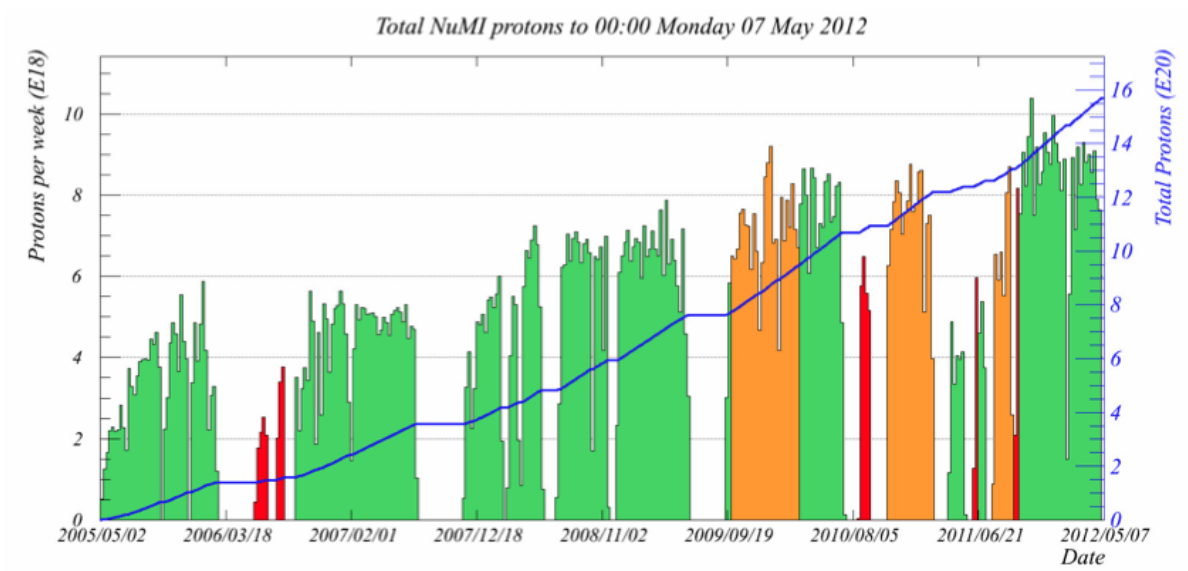

Figure 3.7: The total POT of data collect during the time of operation of MINOS between 2005-2012. Green represents $v_{\mu}$ running, orange represents the $\bar{v}_{\mu}$ mode running and red is for pseudo-high energy beam. 


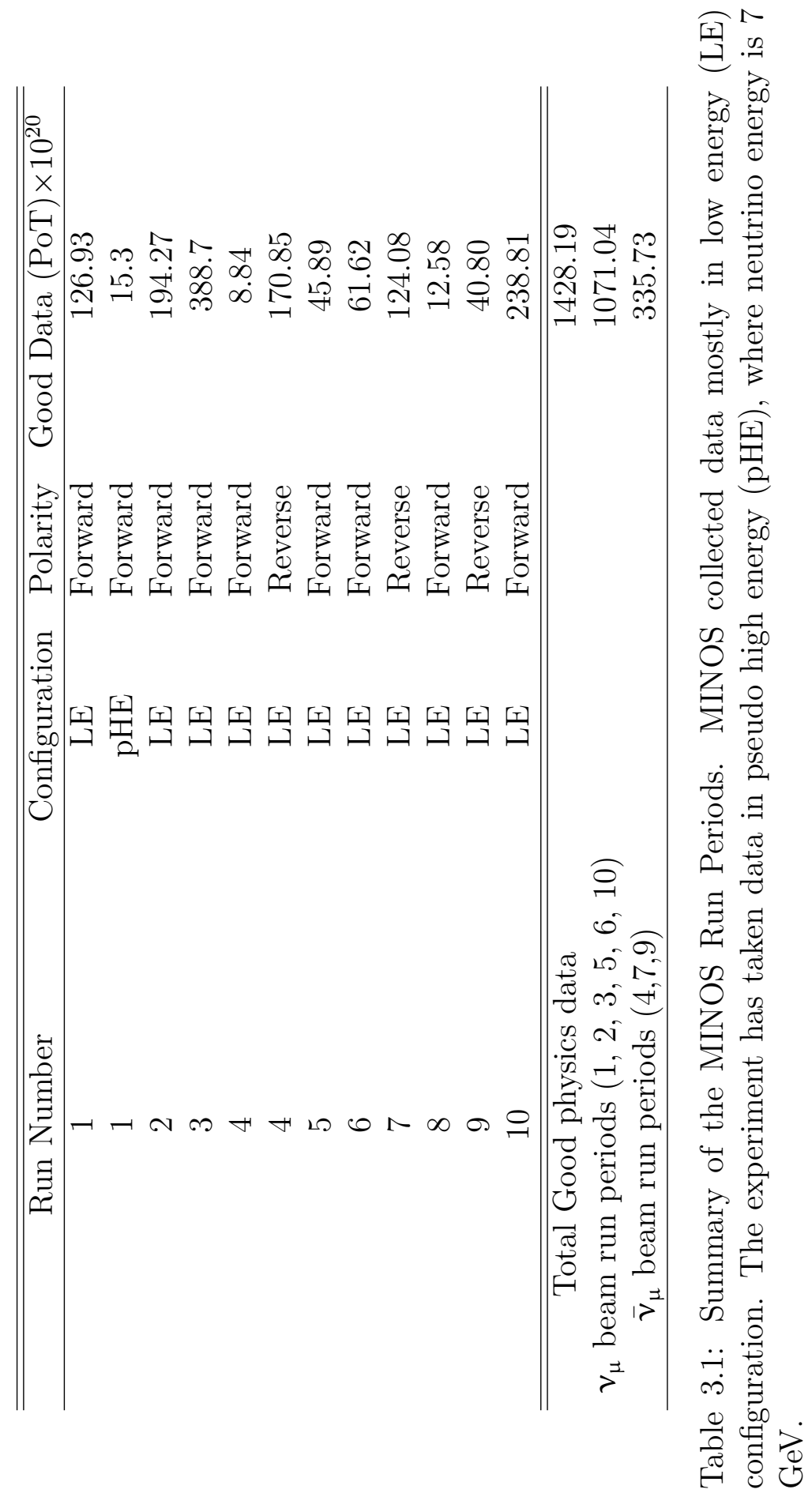




\subsubsection{Results of the MINOS Combined Analysis}

The combined analysis includes the combination of disappearance, appearance results using the entire beam and atmospheric data set [31]. This analysis is sensitive to the value of $\theta_{13}$, mass hierarchy, octant of $\theta_{13}$ and the value of $\delta_{\mathrm{CP}}$. As all the neutrino experiments are entering into a precision era, it is very important to know all of the unknowns. The best fit values of the oscillation parameters for this analysis are $\left|\Delta m_{23}^{2}\right|=2.37_{-0.07}^{+0.11} \times 10^{-3} \mathrm{eV}^{2}$ and $\sin ^{2} \theta_{23}=0.43_{-0.05}^{+0.19}$ for Inverted Hierarchy (IH) and $\left|\Delta m_{23}^{2}\right|=2.34_{-0.09}^{+0.09} \times 10^{-3} \mathrm{eV}^{2}, \sin ^{2} \theta_{23}=0.43_{-0.04}^{+0.16}$ for Normal Hierarchy $(\mathrm{NH})$.
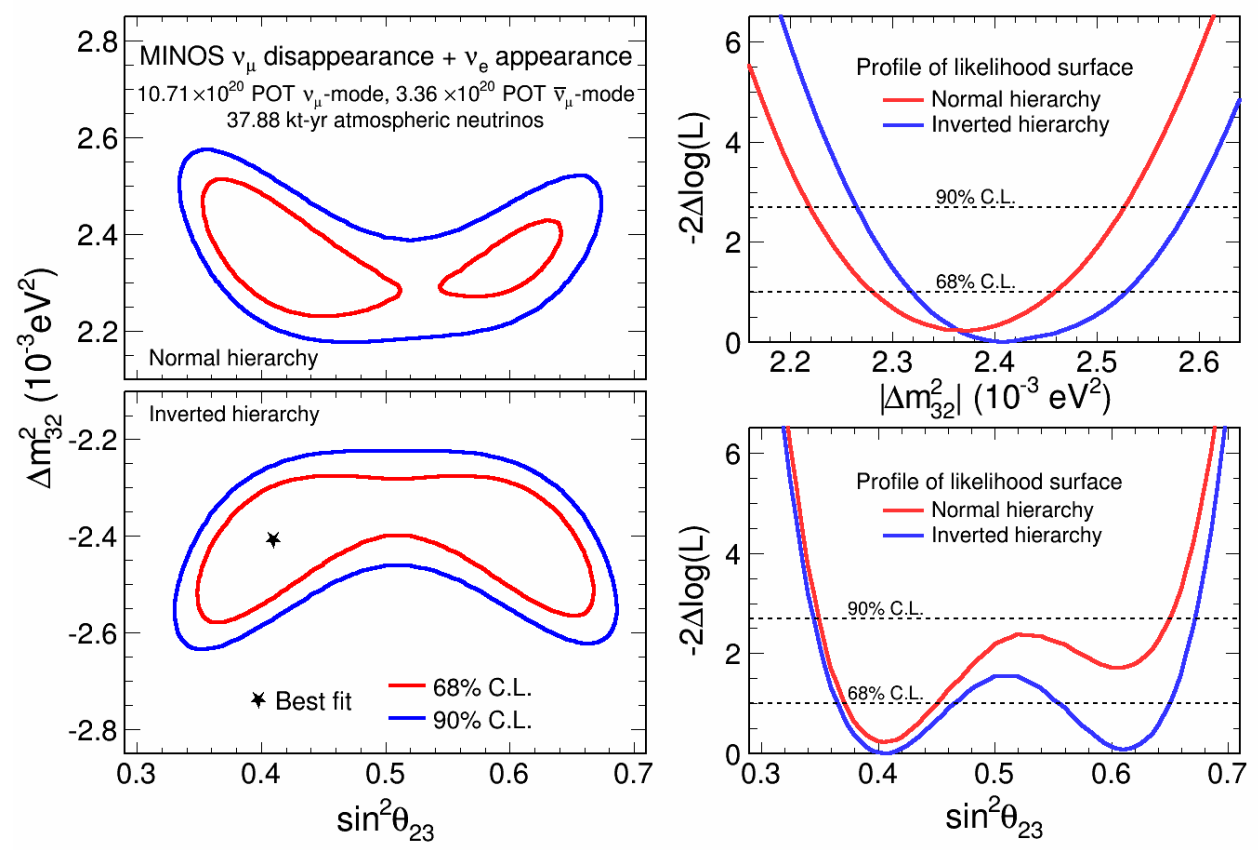

Figure 3.8: Multi-panel contour and profile plots from combined fit to $v_{\mu}$ disappearance and $v_{\mathrm{e}}$ appearance data. Left panels shows showing $68 \%$ and $90 \%$ confidence limits in $\left(\Delta m_{32}^{2}, \sin ^{2} \theta_{23}\right)$ calculated for normal hierarchy (top) and inverted hierarchy (bottom). Right panels show loglikelihood profiles for each hierarchy plotted for $\Delta m_{32}^{2}$ (top right) and $\sin ^{2} \theta_{23}$ (bottom right) 31. 


\subsection{The MINOS Detectors}

The two functionally identical ND and FD are tracking, sampling calorimeter detector having alternate layers of steel and scintillator. The Near Detector is 980 tones and situated at $110 \mathrm{~m}$ underground, at this point neutrino beam is intense and yields 10-15 neutrino event in every spill. The FD is 5400 tones and situated $705 \mathrm{~m}$ underground (2070 meters-water-equivalent). Neutrino flux at this point is much lower than Near Detector and about 2-3 neutrino interaction happens in a day.
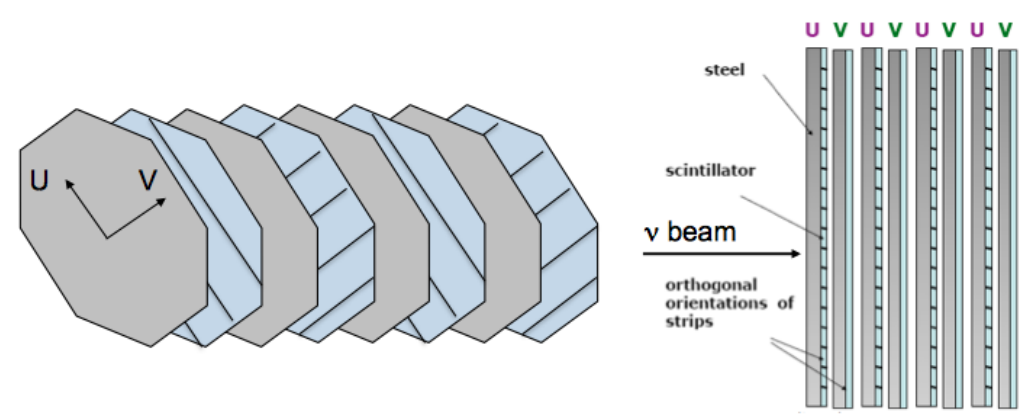

Figure 3.9: The schematic representation of the MINOS detector plane, the alternating plane configuration required for 3 -D reconstruction and the beam direction is shown [76].

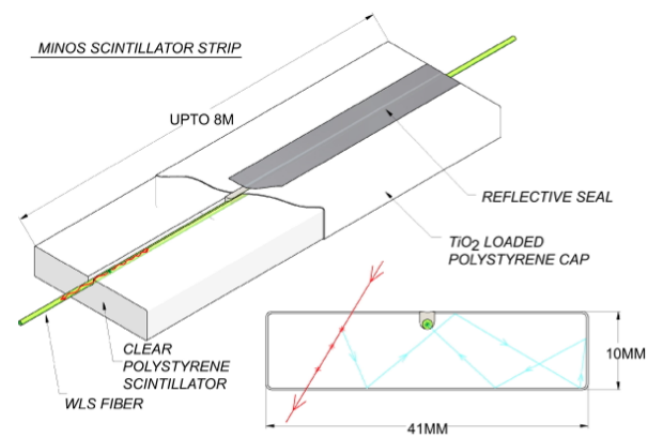

Figure 3.10: The detailed view of the MINOS scintillator strip, when MIP (minimum ionising particle) pass through the scintillator photons are produced, which then collected in wavelength shifted cable and transported to the photomultiplier tube at the edge of the detector [76]. 


\subsubsection{The Near Detector}

The Near Detector consists of 282 steel planes of $2.54 \mathrm{~cm}$, in which first 120 planes have scintillator mounted on it and in these 120 planes, every fifth plane is fully covered with the plastic scintillator and rest of them are partially covered around the beam axis. Rest of the planes out of 282, every fifth plane is fully covered with scintillator and these part of detector is called spectrometer region. The plastics scintillator is $1 \mathrm{~cm}$ thick and 4 $\mathrm{cm}$ wide strips. The strip direction in every plane is in an alternating way (orthogonal to each other) which allow to do a 3-dimensional reconstruction. The fully instrumented section of the detector used to study the hadronic shower energy measurement, whilst the partially instrumented spectrometer region is purely for muon tracking. The entire detector is magnetized with a magnetic field of $1.3 \mathrm{~T}$, which helps to distinguish the positively and negatively charged particles.

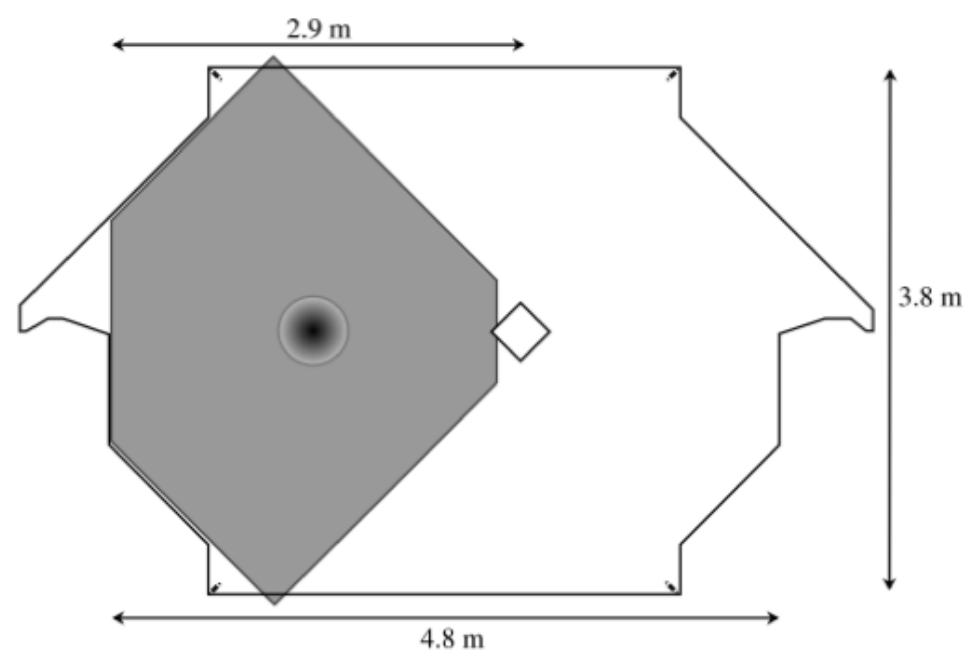

Figure 3.11: A typical ND partially instrumented plane. The shaded grey color represents the covered scintillator portion. For a fully instrumented region the plane is fully covered with scintillator plane. The center diamond shows the coil and the dark circle represent the beam center [76]. 

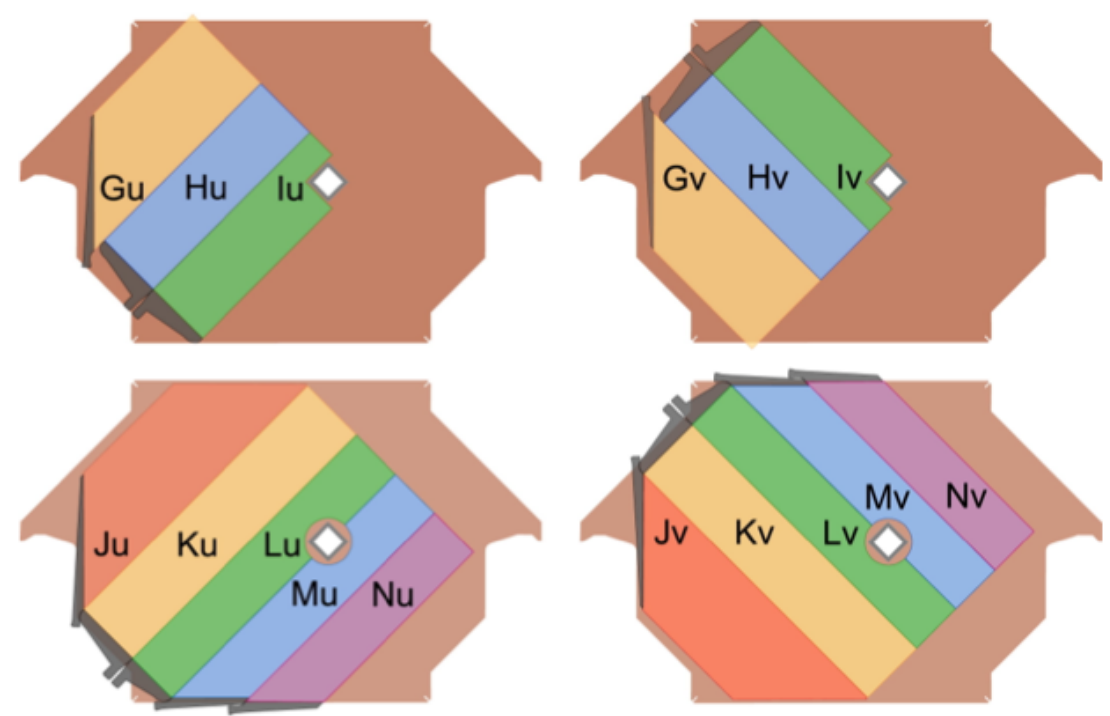

Figure 3.12: The different configuration of scintillator strip module on the Near Detector plane [76].

\subsubsection{The Far Detector}

The Far Detector consists of 486 octagonal steel planes, which are $8 \mathrm{~m}$ edge to edge. The Far Detector is divided longitudinally into two super modules and the cosmic ray event rate is around $0.5 \mathrm{~Hz}$ at this depth. The Far Detector has series of scintillator plane which is placed horizontally over the detector (veto-shield), which helps to identify the cosmic ray muons. The Far Detector's magnetic field is $1.4 \mathrm{~T}$, each plane is fully covered with 192 scintillator strip. 

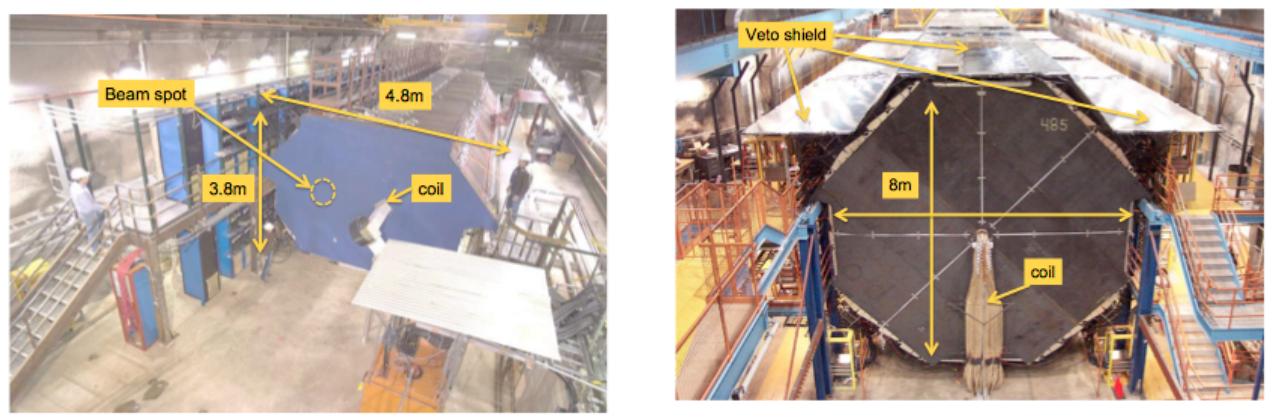

Figure 3.13: Near Detector at Fermilab (left) and Far Detector at Soudan Underground Laboratory at Minnesota (right).

\subsubsection{The Calibration Detector}

The calibration detector is a prototype detector, which helps to measure the absolute energy response of the MINOS near and Far Detectors. The calibration detector consists of 60 steel planes of dimension $2.5 \mathrm{~cm} \times 1 \mathrm{~m} \times 1 \mathrm{~m}$. This detector was operated during the period 20012003 at CERN using the different particles $\left(\pi^{ \pm}, \mu^{ \pm}, \mathrm{e}^{ \pm}, \mathrm{p}\right)$ of ranging energy $0.2-10 \mathrm{GeV}$ from the CERN's Proton Synchrotron accelerator.

\subsubsection{The Magnetic Field}

The MINOS detectors are magnetized. Magnetic field is used to identify the charge of muon coming out of the neutrino interaction thus able to distinguish neutrinos and antineutrinos. Each detector is magnetized using current carrying coils which passes through a hole cut in the detectors. The FD coils has 190 turns, which is made from Teffelon-insulated stranded copper wire and uses a current of $15.2 \mathrm{kA}$. This can produce an average toroidal magnetic field of $1.3 \mathrm{~T}$.

For the Near Detector the coil hole is situated $55.8 \mathrm{~cm}$ offset from the centre of the detector. The detector plane is placed in such a way that the beam centre is half way between the coil hole and left vertical edge of the detector, this avoids lot of track going through the coil hole. Due to the different shape of the ND (squashed octagon) a current of $45 \mathrm{kA}$ is required to achieve the required field [76]. 

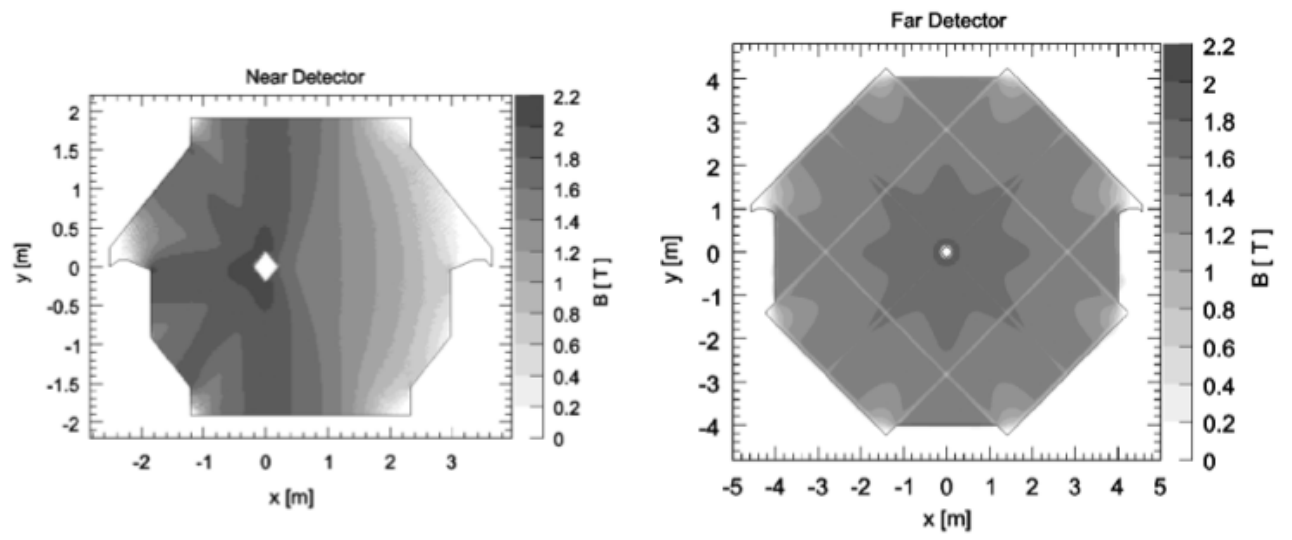

Figure 3.14: Near Detector magnetic field map (left) and Far Detector magnetic field map (right). The grey scale strength of the magnetic field is calculated by finite element analysis using 3D models. Figure taken from [76].

\subsection{Data Acquisition and Trigger System}

The data acquisition system (DAQ) for Near Detector and Far Detector are functionally identical. System consists of appropriate front-end software accommodating the differences in the front-end electronics of the two detectors. The DAQ system is designed to continuously read out the front-end electronics in a un-triggered, dead time free manner and transfer the data to computers where the built in software algorithm selects the events of interest and perform monitoring and calibration tasks [77]. 


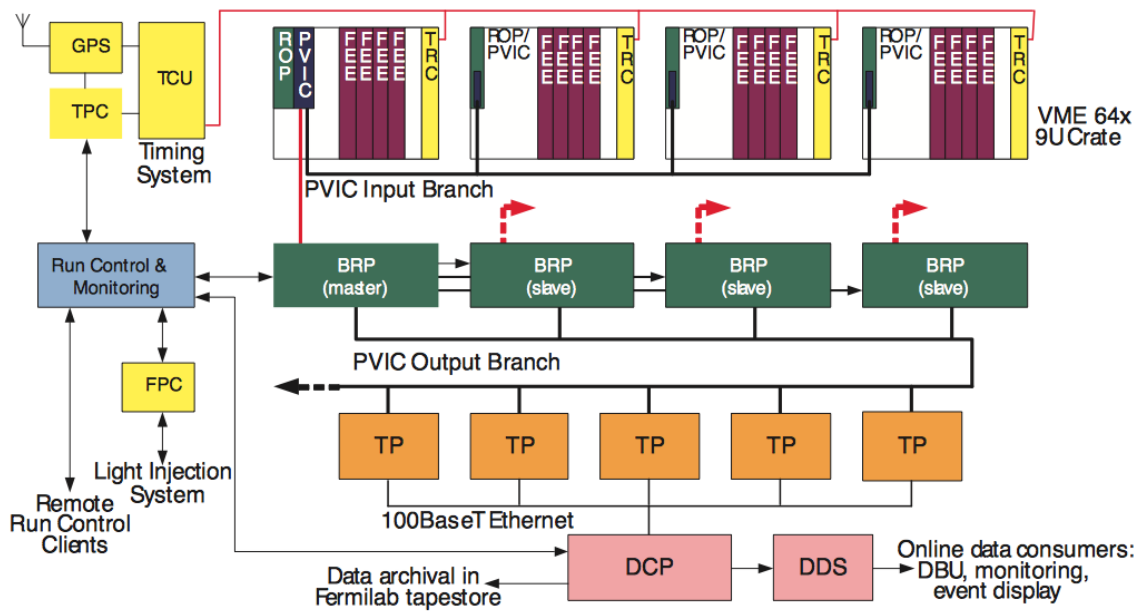

Figure 3.15: A Schematic representation of the Far Detector DAQ system. Figure taken from [77].

Though the DAQ reads the data continuously, it writes to the disk only under some particular conditions(trigger). The ND is triggered by the beam spill from the accelerator at Fermilab. The Far Detector cannot be triggered by the spill since it is not at Fermilab. The Far Detector receives the GPS timestamp of the beam spill from the Near Detector via the internet. The Trigger Processors (TP) in the DAQ system perfumes number of processing task on a time frame of data and also applies software triggering algorithms to locate the events of physics interest. The output from a trigger is called a snarl, can gather multiple events (especially the spill trigger). The primary triggers used in MINOS fall into three categories: special triggers for debugging and calibration, bias-free triggers based on spill signals or spill times to gather beam events and triggers based on the clustering of hits in the detector to gather out-of-spill events.

1. Spill triggers : At the ND, each digitization that occurs within the spill gate is tagged by front-end electronics. These are identified and extracted and output as a single spill event.

2. Remote spill trigger : At FD, a direct spill trigger is not available, 
the ND GPS system is used to generate the timestamps of all the signals and transfer to FD via internet.

3. Fake remote spill trigger : These fake spill times are also generated randomly between the actual spills to provide random sampling of the detector activity.

4. Plane trigger : This is used to write out events when $M$ detector plane in any set of $N$ contiguous planes contain at least one hit. For physics analysis $M=4$ and $N=5$.

5. Energy trigger : This is used to write out events when $M$ contiguous plane of the detector have summed raw pulse height greater than $E$ and total of at least $N$ hits in those plane. Nominally $M=4, E=$ $1500 \mathrm{ADC}$ and $N=6$.

6. Activity trigger : This requires $N$ plane should have activity across the detector, typically $N=20$.

7. Special triggers : There are additional triggers which used for the electronics and detector calibration or debugging.

\subsection{Interactions in the MINOS Detectors}

The MINOS detector gives different topology for the different type of interaction, the most common interaction that happens in the detector when a neutrino interacts is Charged Current (CC) $\nu_{\mu}$ event. The CC $v_{\mu}$ events are very important for the precise measurement of $v_{\mu}$ disappearance. The other type of interaction which is common in the detector is a Neutral Current (NC) events, which is important for studying the sterile neutrino search as well as to understand the background for other interactions. The other category of event is $v_{\mathrm{e}} \mathrm{CC}$, which is important for the appearance analysis and measure of the mixing angle $\theta_{13}$.

\subsubsection{Charged Current $v_{\mu}$ Interactions}

The $v_{\mu}$ CC interaction happens when a neutrino interact inside the detector and exchange $W^{ \pm}$boson, the signature of these kind of events in the detector are long $\mu$ track along with a hadronic shower. In MINOS a $1 \mathrm{GeV}$ muon typically travels across 25 planes, the long track muons are 
the key feature of this kind of interaction. Comparatively a large portion of the short tracks are part of NC interaction and those are the main background for this type of interaction.
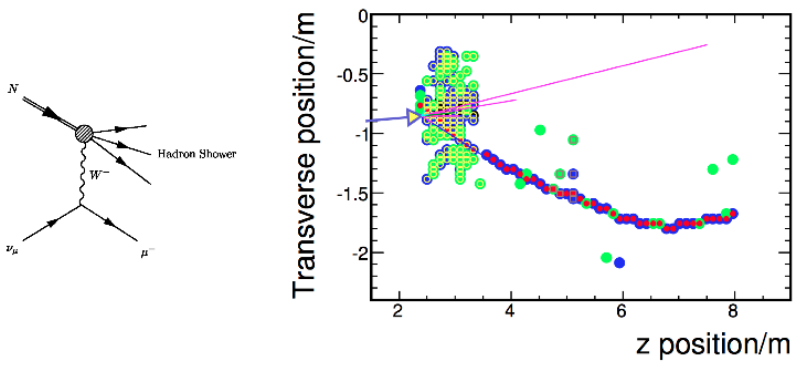

Detected energy depositions

- Deposition $<2.0$ pe

- $2.0<$ Deposition $<20.0$ pe

- Deposition $>20.0$ pe

Reconstruction

- Reconstructed track hit

Monte Carlo Truth

$\rightarrow$ Initial $v_{\mu}$

$\boldsymbol{\mu}^{-} \quad-\pi^{ \pm}$

Figure 3.16: Feynman diagram for a $v_{\mu}$ CC interaction (left). The corresponding reconstructed event in the MINOS detectors, a long muon track is clearly visible. The dots represent the reconstructed position of the scintillator strip with different energy deposition (right).

\subsubsection{Neutral Current Interactions}

Neutrino neutral current interactions are the weak interaction mediated by $\mathrm{Z}^{0}$ boson. The characteristic feature of $\mathrm{NC}$ events are hadronic shower in the detector, along with an outgoing neutrino which leaves the detector.
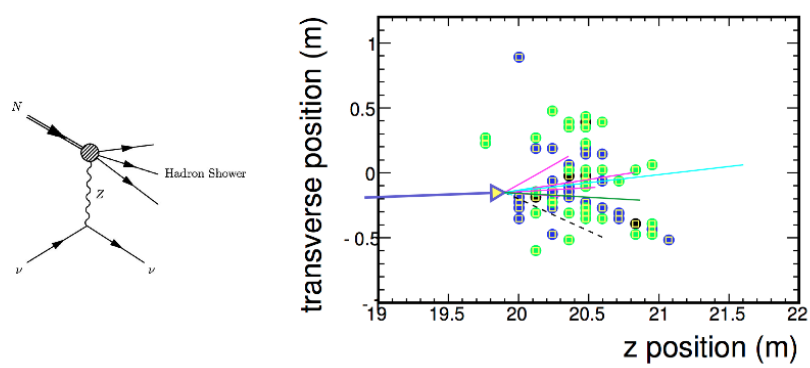

Detected energy depositions

- Deposition $<2.0$ pe

- $2.0<$ Deposition $<20.0$ pe

- Deposition $>20.0$ pe

Reconstruction

- Reconstructed track hit

Reconstructed shower hi

Monte Carlo Truth

$\rightarrow$ Initial $v_{\mu} \quad \cdots$ Outgoing $v$

$\pi^{ \pm} \quad-n^{0}$

Figure 3.17: Feynman diagram for a NC interaction (left). The corresponding reconstructed event in the MINOS detectors, the hadronic shower is clearly visible. The dots represent the reconstructed position of the scintillator strip with different energy deposition (right). 
A typical $\mathrm{NC}$ event in the detector deposits $1 \mathrm{GeV}$ of energy with an average of 10 hits in the scintillator strip. The cross section of NC events are identical for three active flavour neutrino and the $\mathrm{NC}$ event rate in the detector are not affected by the standard three flavour oscillation. So any observation of deficit in the NC events in the Far Detector could be a hint for a fourth type of neutrino.

\subsubsection{Charged Current $v_{\mathrm{e}}$ Interactions}

The $v_{\mathrm{e}}$ CC interaction gives compact electromagnetic shower surrounded by a sparser hadronic shower in the detector. The source of $v_{\mathrm{e}} \mathrm{CC}$ in the MINOS detector are, the "intrinsic $v_{\mathrm{e}}$ ", (interaction of $v_{\mathrm{e}}$ coming from the beam $1.8 \%$ in $v_{\mu}$ and $1.9 \%$ in $\bar{v}_{\mu}$ mode) and the $v_{\mathrm{e}}$ which is oscillated from the $v_{\mu}$. The intrinsic $v_{\mathrm{e}}$ events are having higher energy than the appearance $v_{\mathrm{e}}$. For the appearance $v_{\mathrm{e}}$ the energy peaks around $1.4 \mathrm{GeV}$ which is associated with the maximum of $v_{\mu}$ oscillation in the MINOS baseline. The main background for the $v_{\mathrm{e}}$ events are the NC events. The granularity of the MINOS detectors are not designed to select these type of events and a special algorithms were used to select the $v_{\mathrm{e}}$ events [78].
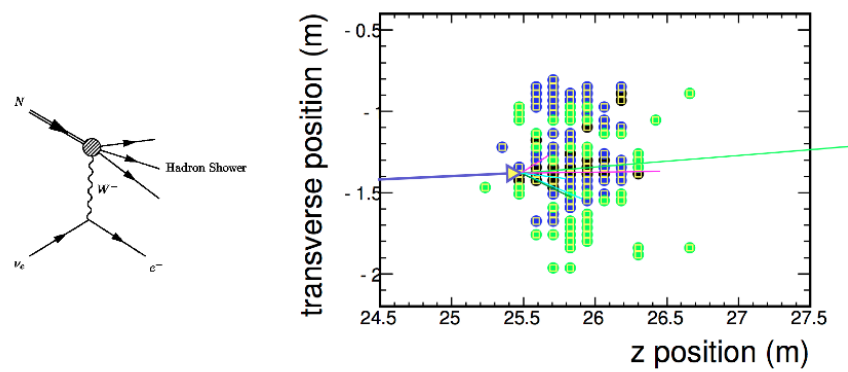

Detected energy depositions

- Deposition $<2.0 \mathrm{pe}$

$2.0<$ Deposition $<20.0$ pe

- Deposition $>20.0$ pe

Reconstruction

Reconstructed track hit
Reconstructed shower hit

Monte Carlo Truth

$\rightarrow$ Initial $v_{\mu}$

$\mathrm{e}^{ \pm} \quad \mathrm{\pi}^{ \pm} \mathrm{p}^{0}$

Figure 3.18: Feynman diagram for a $v_{\mathrm{e}}$ interaction (left). The corresponding reconstructed event in the MINOS detectors, the signature is a compact electromagnetic shower. The dots represent the reconstructed position of the scintillator strip with different energy deposition (right).

\subsection{The MINOS+ Experiment}

The MINOS experiment has been operated for 7 year starting form 2005 to 2012 April. The MINOS detectors are still functioning in the 
NOvA era in the medium energy NuMI beam [79]. The energy of the neutrino spectrum in MINOS+ is around $6 \mathrm{GeV}$ both for $\nu_{\mu}$ and $\bar{v}_{\mu}$, also the beam composition is different [23]. With this higher energy and intense neutrinos MINOS+ will able to collect more neutrinos events, 10000 $v_{\mu} \mathrm{CC}$ and $3000 \mathrm{NC}$ events in the first three year of running. MINOS+ will probe different physics with the more statistics data, which includes sterile neutrinos search, search for extra dimension, non-standarad neutrino interaction etc. All new physics results will be adding valuable input to the upcoming neutrino programs. 


\section{4 \\ Event Reconstruction, MC Simulation and Calibration}

The MINOS detectors record neutrino interactions from the NuMI beam spills, muons induced interaction in the surrounding rock and the cosmic ray muons whose tracks have traversed the detector volume. Neutrino events are reconstructed using low level detector hit information (topology) and timings to produce muon tracks and hadronic showers which are then combined to get the complete information of the neutrino interaction. The primary signature of $\mathrm{CC} \nu_{\mu}$ interaction in the MINOS detector is the presence of a muon track with a contained vertex. Event reconstruction proceeds by taking candidate reconstructed objects and passing them through an ordered set of algorithms whereby an event is successively reconstructed. The reconstruction strategy is outlined and described in this chapter.

\subsubsection{Digit Formation}

The raw data (or MC simulation) in a particular time window are converted into a digitized measurement of pulse height from the recorded scintillation light by the read-out electronics. Each digit contains information about the timestamp and the list of possible strip ends associated with it. The Far Detector and Near Detector spectrometer regions are multiplexed, so that a demultiplexing algorithm is required to identify which out of eight (FD) and four (ND spectrometer region) strip end associated with the scintillation light [80]. The Far Detector reads out from two ends, so, the algorithm finds the digit combinations such that the two digits are identified as coming from the same strip. 


\subsubsection{Strip Formation and Slicing}

Strip objects are those digits which represent a single energy deposition in the scintillator strip. The strip objects are formed by combining the digits recorded and originating at same time from each end of the scintillator strip at Far Detector and from the single end in the Near Detector. In the Near Detector multiple neutrino interactions happen per spill, because of this reason it is very difficult to separate individual showers and events. To simplify the reconstruction process at ND, the snarl is divided into slices. A slice is collection strips which are temporally and spatially close and likely to be originating from a single neutrino interaction. In the calorimeter region the strips having charge deposition greater than two photoelectron with time difference smaller than $20 \mathrm{~ns}$ and a total time window of less than $300 \mathrm{~ns}$ are typically formed into slices. If the slice formed with two strips are spatially separated by more than $1 \mathrm{~m}$ in longitudinal direction, then it is split into two slices. The calorimeter strip with less than 2 photo electron and spectrometer strips are combined into a suitable slice using the timing information. For FD, the snarls are divided into slices, but in Far Detector typically one event occurs in a spill.

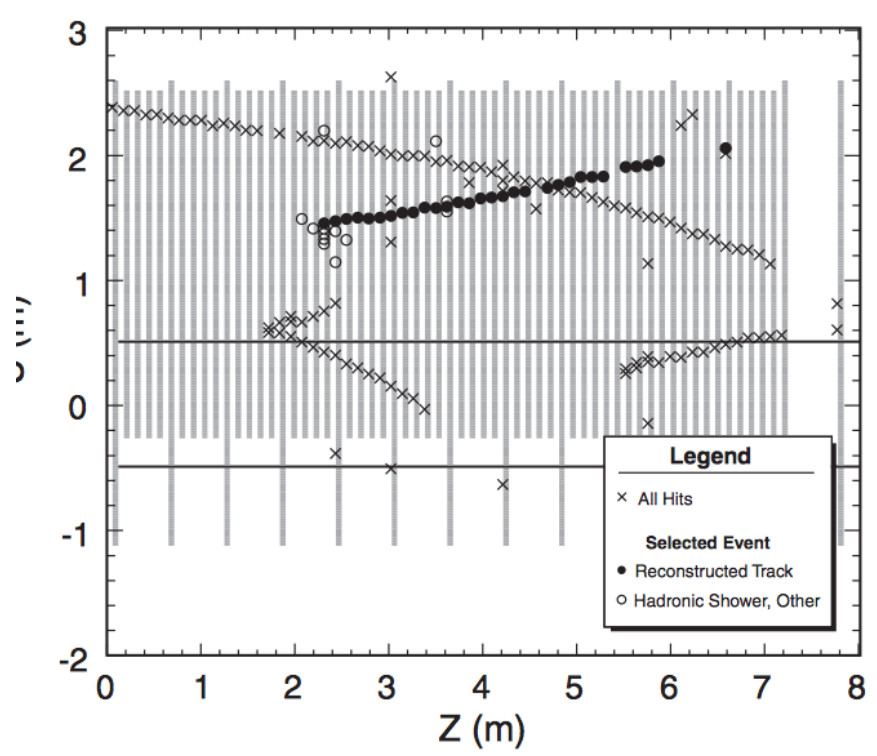

(a) 


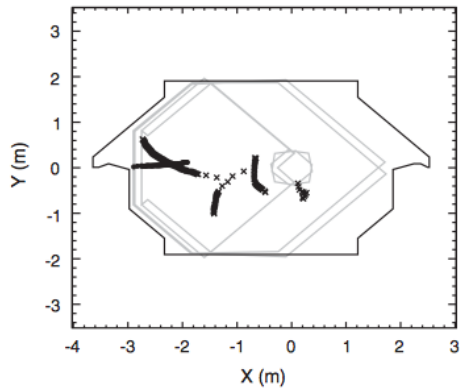

(b)

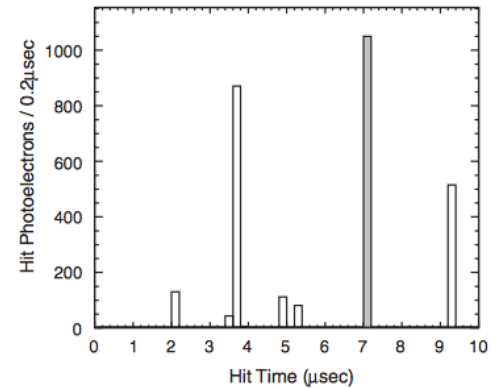

(c)

Figure 4.1: One beam spill observed in the Near Detector. Neutrinos are incident from the left and only the upstream section of the detector is shown. Grey vertical bars indicate the scintillator coverage. The timing and topological pattern of hits in the detector has been used to reconstruct and select an event containing a $5.6 \mathrm{GeV}$ muon and a $2.6 \mathrm{GeV}$ hadronic shower. Beam's eye view of the detector is shown in (b), along with the reconstructed horizontal and vertical positions of track hits in each detector plane. Figure (c) shows the detector signal as a function of time, with signals from the selected event shaded [81].

\subsubsection{Track Reconstruction}

After splitting the snarl into different slice, the next step is to apply the track finding algorithm. Hough transform algorithm is used to find the track segments consisting of several hits in an approximate line across the detector [82]. A track finder is used to join the segment and form a "seed track". These seed tracks are basic structure of a muon track. Kalman filter algorithm is used for the track fitting [83 86]. Kalman filter is a recursive equations that enables the state of a dynamical system to be estimated from a series of incomplete and noisy measurement. Kalman filter moves forward and backward along the track and estimate the state of muon at each point along the track. Kalman filter takes into account the effects such as noise and multiple scattering in the calculation. It also includes the information about the curvature in the magnetic field. The Kalman filter updates the state vector of the muon after the two passes of the fit. The state vectors contains the full information about the track at detector the plane and it specifies the transverse positions ( $u$ and $v$ ) of 
the muon, transverse direction of muons $\left(\frac{d u}{d z}\right.$ and $\left.\frac{d v}{d z}\right)$ at the track vertex, the reconstructed ratio of charge sign to momentum $q / p$ as determined from the curvature of the track in the magnetic field and the uncertainty associated with the vertex $q / p(\sigma(q / p))$ is extracted from the final noise covariance matrix at the track vertex.

For the track which ends in the detector, a second, more accurate measurement of momentum is obtained from the range. This measurement has a resolution of $4.6 \%$ and used for all tracks that do not exit the detector or end in coil hole region. This particular task is performed by a swimmer, which swims the muons backward along the track from the track end to the beginning and summing the energy deposited in the steel and scintillator using the GEANT3 [87] simulation.

\subsubsection{Shower Reconstruction}

The shower energy is reconstructed from the clusters of strips that are localised in space and time. Hits which are part of muon track and have deposited energy more than a muon would have deposited are added to the shower after the muon part is removed. Only hit with energy deposition more than two photoelectrons are considered since low pulse height regions are difficult to model. It is also difficult to reconstruct showers from the topology of the event as the granularity of the detector is too coarse. In MINOS, we calculate the shower energy in two methods which are described below.

The shower energy is calculated by summing the energy deposited by all of the constituent hits (the energy deposited in the steel is recovered). This method is called calorimetric shower energy calculation. The resolution of this method for hadronic shower is $56 \% / \sqrt{E} \oplus 2 \%$ and for the electromagnetic shower it is $21.4 \% / \sqrt{E} \oplus 4 \%$ [76]. The other method to calculate the shower energy is using a $\mathrm{k}$ - nearest neighbour (kNN) algorithm. The kNN algorithm is a multivariate neural network technique, which improves the energy resolution of the showers. It makes use of the border range information and also the information from the events as a whole. The kNN reconstruct the shower energy using the amount of deposited energy as well as the spatial distribution information of energy inside the shower [88]. For a $D$ - dimensional $\mathrm{kNN}$, a set of $D$ input variable are considered, which characterizes the properties of shower energy 
and correlate with the true energy.

The required elements for the $\mathrm{kNN}$ algorithm are the training set, variables to build multi-dimensional feature, the number of nearest neighbour and energy correction function. Every event whose energy is to be calculated is compared with the training set and $\mathrm{k}$ closely matching events are picked up. The number of nearest neighbour $k$ is the only adjustable parameter once the variables are selected, as $k$ increases the accuracy of the measurement also increases as more neighbours add more information. The definition of "nearest neighbours" is in terms of the Euclidean distance between points, where the input variables $x_{i}$ is the Monte Carlo events whose true parameters are known and $y_{i}$ is the events which we are looking for, the squared distance between the two is given by,

$$
\Delta s^{2}=\sum_{i=1}^{D} \frac{\left(y_{i}-x_{i}\right)^{2}}{\sigma_{i}^{2}},
$$

where, $\sigma_{i}$ is the standard deviation of the variable. The normalization by the standard deviations is necessary to ensure consistency in the scales of the variables. Otherwise the distance metric would be dominated by whichever variable varied over the largest range. The scale differences can easily be large since the variables are not necessarily in the same units. The shower energy is estimated by finding the $k$ closest training events in the $D$ - dimensional space formed by the input variable. The output estimate of energy is the mean true shower energy of these events. The input variables are optimized for getting the best oscillation results and the input variables are listed below,

- Sum of the energies of the first two showers in the event.

- The number of distinct detector planes that contain hits from this shower.

- Sum of the energies of all showers with vertex within $1 \mathrm{~m}$ of the track vertex. 


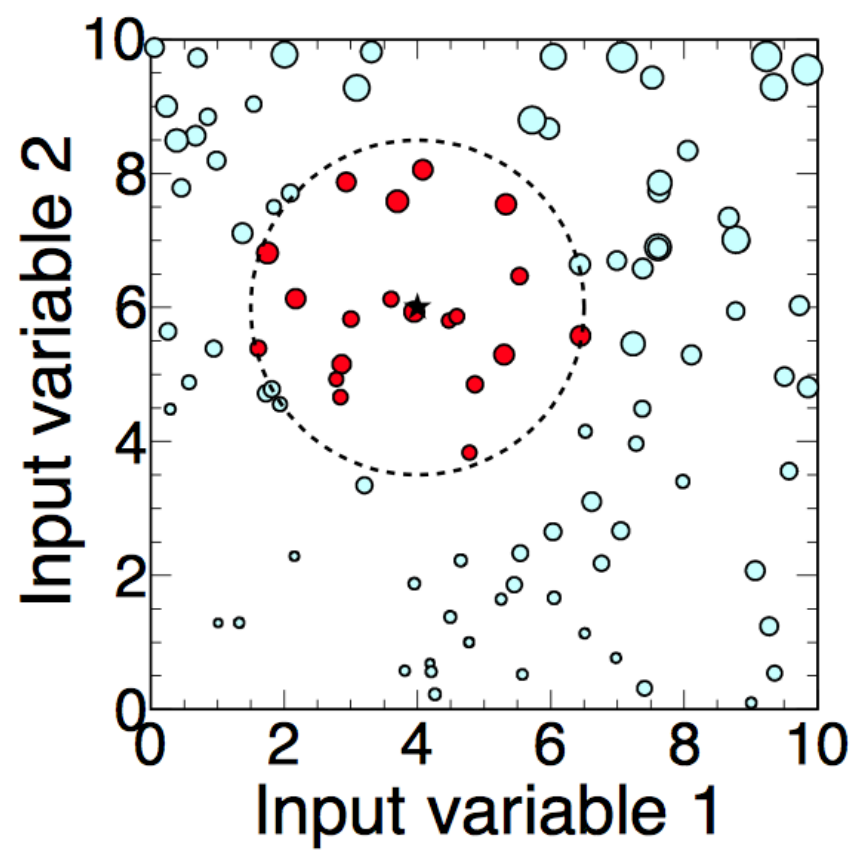

Figure 4.2: Cartoon of the kNN algorithm. Training points neighbouring the input point (the star) and their selected energies (represented by the diameters) are averaged.

\subsubsection{Formation of Event}

At the final stage the neutrino events are reconstructed by combining the reconstructed track and reconstructed shower. Event object is the sum of the vertex shower and most energetic track. The track with clearly defined vertex is one of the condition to get the $\nu_{\mu}$ and $\bar{\nu}_{\mu} \mathrm{CC}$ events, which we use for the disappearance analysis.

\subsection{Simulation}

The MINOS MC simulation is a multi-stage process. The two detector design of the experiment greatly reduces the analysis independent of the simulation, but still it is very important to simulate the data events as accurate as possible. The chain of MC simulation starts with the prediction of neutrino flux from the NuMI beam using the FLUGG Monte 
Carlo generator [89-91]. This package incorporates a geometry modelled by GEANT3 92 based on a GNUMI framework into FLUKA 933 simulation, which simulates the interaction of protons on target (hadron production), propagation of the particles, re-interaction of the particle and the decay of the particle in the decay pipe volume. The simulation begins with the production of mesons by the proton beam on the target. These mesons are propagated through the magnetic horns, decay pipe and all other components at the downstream part of the NuMI. Every neutrinos produced by the hadron decay are stored and later used to simulate the neutrino interaction. The probability of neutrinos which reaches in the detector can be calculated based on the position and momentum information of the neutrinos produced. Every neutrinos are forced to go to the detectors with a weight calculated using the probability to actually do so. The resulting neutrino flux is then pass into the MINOS detector simulation.

Neutrinos produced from the flux are sampled using a technique called "importance weighting" to reduce the number of low energy particles produced. The simulation produces too many low energy mesons than high energy mesons, so it is difficult to collect a high statistics of higher energy mesons. To increase the higher energy events, a $1 \mathrm{GeV}$ tracking threshold is imposed on the mesons, since these mesons produce neutrino energy of approximately $500 \mathrm{MeV}$ or lower, which is the lowest energy neutrino that the MINOS detectors are sensitive to. Even after the threshold imposed there are still too many low energy particles survive. To reduce the space required on disk and speed up the computation process, the pions with less than $30 \mathrm{GeV}$ are importance weighted. After all, the low energy particles which are having energy lower than the threshold are discarded and a weight greater than one is given to the rest of the particles, so that the the total flux after the reweighing remains the same. Then the weight is given by,

$$
W=W_{\text {parent }} \frac{30 \mathrm{GeV}}{\left|P_{\text {total }}\right|},
$$

where, $W_{\text {parent }}$ is the importance weight of the particle's parent(initial protons are assigned a weight 1 ) and $P_{\text {total }}$ is the total momentum of the particle.

The neutrino interactions are simulated using NEUGEN3, it is a widely 
used neutrino interaction generator that produces complete final state particles in a neutrino-nucleus interaction from energy $100 \mathrm{MeV}$ to $100 \mathrm{GeV}$. The Fermi gas model with some modification for nucleon-nucleon [94] correlation is used as the nuclear model. The cross section model that NEUGEN uses are quasi-elastic scattering (QE) [95], resonance production(RES) [96], coherent pion production [97] and deep inelastic (DIS) scattering. The hadronisation model used in NEUGEN is AGKY model [98. This model has two components, at the high hadronic invariant mass it use PYTHIA/JETSET [99] and the low invariant mass component uses a KNO model [100]. The intranuclear rescattering as the particle leaves the nucleus are taken into account in NEUGEN using INTRANUKE model [101]. To simulate the underlying physics, the resultant events from the simulation are passed through GEANT - 3 based detector simulations. Then the particles are transported through the detector geometry and calculates and store the energy deposition by strip to strip. After the energy deposition, a Photon Transport program calculates the number of photon in the scintillator based on the energy depositions and these photons are then transported through a wavelength shifting (WLS) fiber onto a PMT cathode where photons are converted it into photoelectrons. At this stage simulation take into account of the behaviour of PMT and electronics including the nonlinearity, cross-talk, trigger etc. This helps to include the best knowledge about the light levels, attenuation, gain and non-linearity in the simulation. When we generate the $\mathrm{MC}$, a particular date during the data taken is assigned, so that the calibration constant from those dates and time are properly applied. Later when calibrations are reapplied and each $\mathrm{MC}$ is re-calibrated using the same data that was used to produce it. At this point, the simulation is similar to the real data and both are handled in the same way.

\subsection{Calibration}

The MINOS calibration is a multistage process in which the raw pulse height is converted into a corrected signal. The calibration corrects for the scintillator light output variations as well as non-uniformities of light transmission, collection in the fibers, PMTs, and readout electronics. Calibration uses a optical light injection system which measures the response of the read out system and cosmic muons, which measures and corrects for the scintillator response. The raw pulse height $Q_{\mathrm{s}, \mathrm{x}, \mathrm{t}, \mathrm{d}}$ in strip s, po- 
sition $x$, time $t$ and $d$ detector is converted into a corrected signal $Q_{\text {corr }}$ by applying a multiplicative calibration constant from each stage. It can be expressed as,

$$
Q_{\text {corr }}=Q_{\text {raw }} \times D(d, t) \times L\left(d, s, Q_{\text {raw }}\right) \times S(d, s, t) \times A(d, s, x) \times M(d)
$$

where, $D$ - is the drift correction to account for scintillator response, PMT and electronics response changing with temperature and age (drift calibration).

$L$ - The linearity calibration constant which linearizes the response of each channel with pulse-height (linearity calibration).

$S$ - This is the strip-to-strip constant which accounts for the differences in strip to strip and channel-to-channel response (strip to strip calibration). $A$ - The attenuation correction, which describes the attenuation of light depending on event position along each strip (attenuation calibration). $M$-The overall scale factor which converts the corrected pulse height into the same absolute energy unit for all detectors (inter-detector calibration).

\subsubsection{Drift Calibration}

The drift correction $D(d, t)$ is measured daily by determining the median response of the detector to atmospheric muons and comparing that to a reference date. The magnitude of the drift correction is set by a combination of competing effects, the gains of the photo-multiplier tubes, which typically increase over time and the light-level incident at the PMT which typically decreases over time. The overall light-level is affected by the light production in the scintillator, the transmission efficiency to the wavelength shifting fibers, attenuation in the fibers and the quantum efficiency of the photocathode. Over the complete MINOS data set, the gains have increased by approximately $20 \%$ and the light level has decreased by $25 \%$ and we have

$$
D(d, t)=\frac{\text { Median } \operatorname{response}\left(d, t_{0}\right)}{\text { Median } \operatorname{response}(d, t)}
$$

\subsubsection{Linearity}

The linearity correction accounts for the non-linearity in electronics response as a function of ADC pulse height and it is determined from 
information obtained using the Light Injection (LI) system. The LI system pulses each strip over a range of intensities to obtain corrections.

\subsubsection{Strip to Strip calibration}

The intra-detector calibration $S(d, s, t)$ removes differences in response from channel to channel and it is performed using atmospheric muons. The strip to strip calibration accounts for the the serval detector effects like scintillator light yield, wavelength shifting fiber collection efficiency, differences in attenuation in the optical fibres, PMT quantum efficiency and PMT gain etc. Channel-to-channel variation is around 30\% and is approximately due to equal parts $(20 \%)$ variation in light-level and variation in the PMT gain. This calibration reduces a nominal $30 \%$ variation across channels to below $5 \%$. This calibration relates the mean response of the each strip end to the detector average and the calibration factor is given by,

$$
S(s, d, t)=\frac{\text { Mean response of the detector }(d, t)}{\text { Mean response of the Strip end }(\mathrm{s}, \mathrm{d}, \mathrm{t})}
$$

\subsubsection{Attenuation}

The attenuation correction $A(d, s, x)$ for each strip is determined by mapping the response to atmospheric muons as a function of position along the strip. The data fits well to a double exponential and agrees well with earlier test-bench corrections using radioactive sources. The response along an individual strip varies by factor of two and three in the Near and Far Detectors, respectively and the statistical variation along the strip after the attenuation correction is less than $2 \%$. The calibration constant is given by,

$$
A(d, s, x)=A_{1} e^{-x / L_{1}}+A_{2} e^{-x / L_{2}} .
$$

Where, $x$ is the length along the strip and $L_{1}, L_{2}$ are two attenuation lengths. A fit is performed in each strip and resulting parameter are used to correct the data.

\subsubsection{Relative Energy Calibration}

The absolute energy scale of the detectors is determined by measuring the response of the detectors to stopping muons (after the above calibrations have been applied). A segment of muon track with small $d E / d x$ 
variations is chosen to measure the response. The measured response is expressed in terms of a energy unit, called the Muon Energy Unit (MEU). This is a response to a well defined energy depositions at each detectors. The uncertainties in the MEU results in a total $2.1 \%$ uncertainty in the relative energy scale between the Near and Far Detector. The Near and Far detectors uncalibrated and calibrated response are shown in Fig 4.3 .
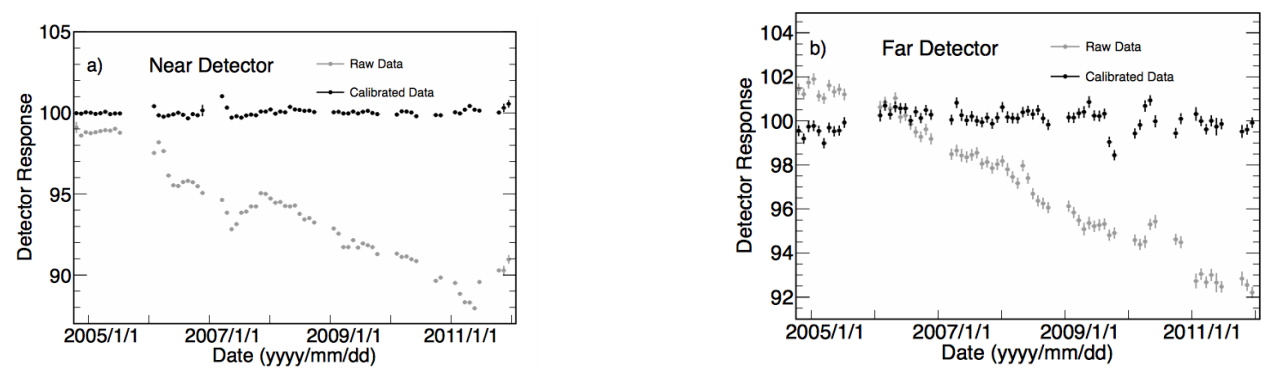

Figure 4.3: The response in MEU for the a) Near Detector and b) Far Detector as a function of time, as measured using cosmic data, before and after applying calibration corrections. Each point corresponds to one month of data and is normalized to the mean MEU value for all run periods. The calibrated MEU value over time is stable to within $0.5 \%$ for Near Detector and within 1.5\% for Far Detector.

\subsection{Absolute Energy Scale}

After all the calibration procedure is done, the Near and Far Detector response are expressed in terms absolute energy scale. Once the absolute energy scale is known the energy response for different particles, like electron, muons, hadrons of certain energy can be determined. CalDet detector which is exposed to the various test beams in CERN is used to measure the absolute energy scale. CalDet measured the response of different particle like electrons, pions, muons, and protons of both charge signs with momenta ranging from $200 \mathrm{MeV}$ to $10 \mathrm{GeV}$. As we know the momentum, charge of the particle prior to the measurement we can use this data to compare with the GEANT3 detector simulations. 


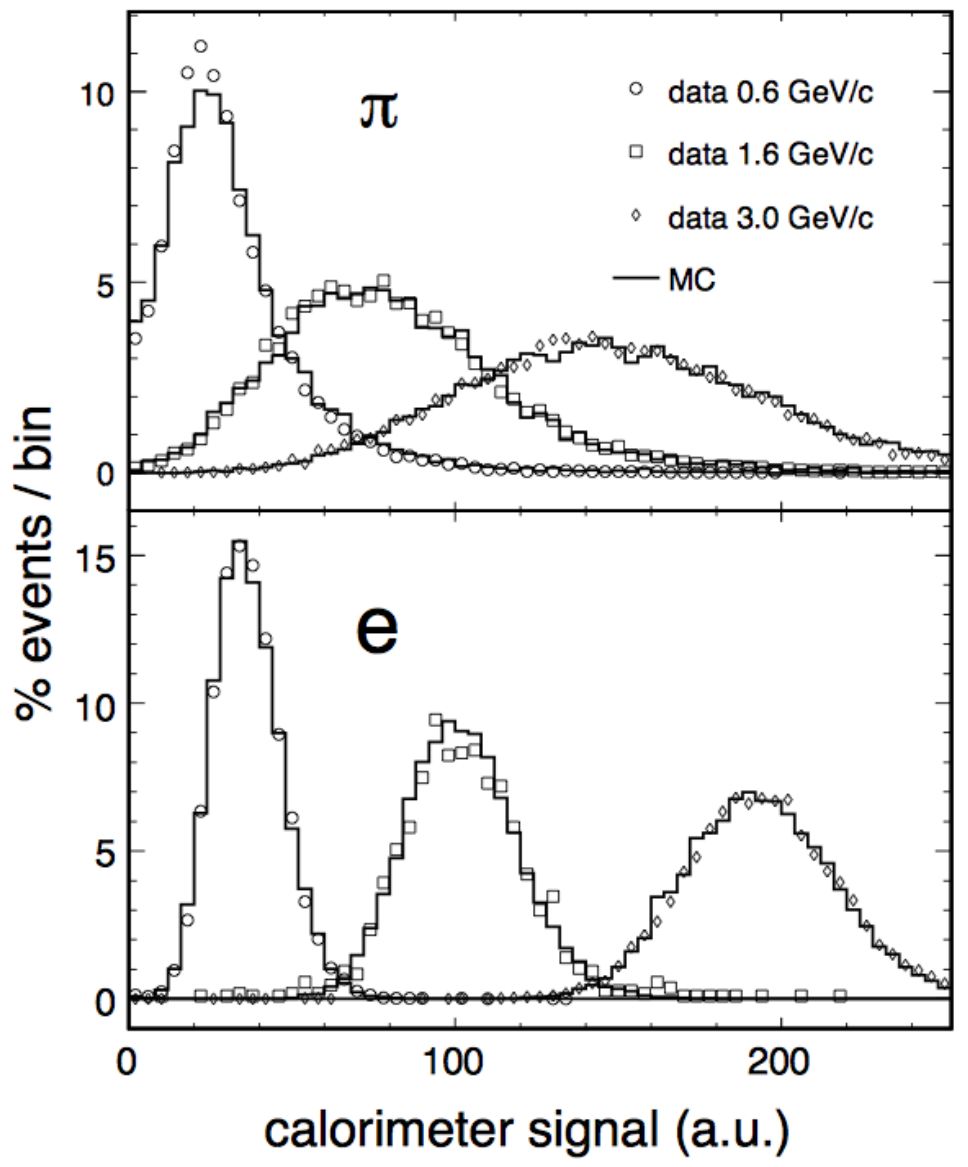

Figure 4.4: MINOS calorimetric response to pions and electrons at three momenta. The calorimeter-signal scale is in arbitrary units. The data (open symbols), obtained from the calibration detector exposure to CERN test beams are compared to distributions from Monte Carlo simulations [76].

The range of stopping muons $(p<2.2 \mathrm{GeV} / \mathrm{c})$ was modeled to better than $3 \%$, thereby benchmarking the combined accuracy of the muon energy loss treatment, beam simulation and beam momentum. The simulated detector response to electrons agreed with the data to better than $2 \%$ [117]. Pion and proton induced showers were reproduced to better than $6 \%$ accuracy with the GCALOR [118] simulation. The energy resolution was adequately reproduced by the simulation and parameterised as 
$56 \% / \sqrt{E} \oplus 2 \%$ for hadron showers and $21 \% / \sqrt{E} \oplus 4 \%$ for electromagnetic showers, where the energy $E$ is in GeV. A summary of the MINOS calibration procedure is shown in Fig 4.5

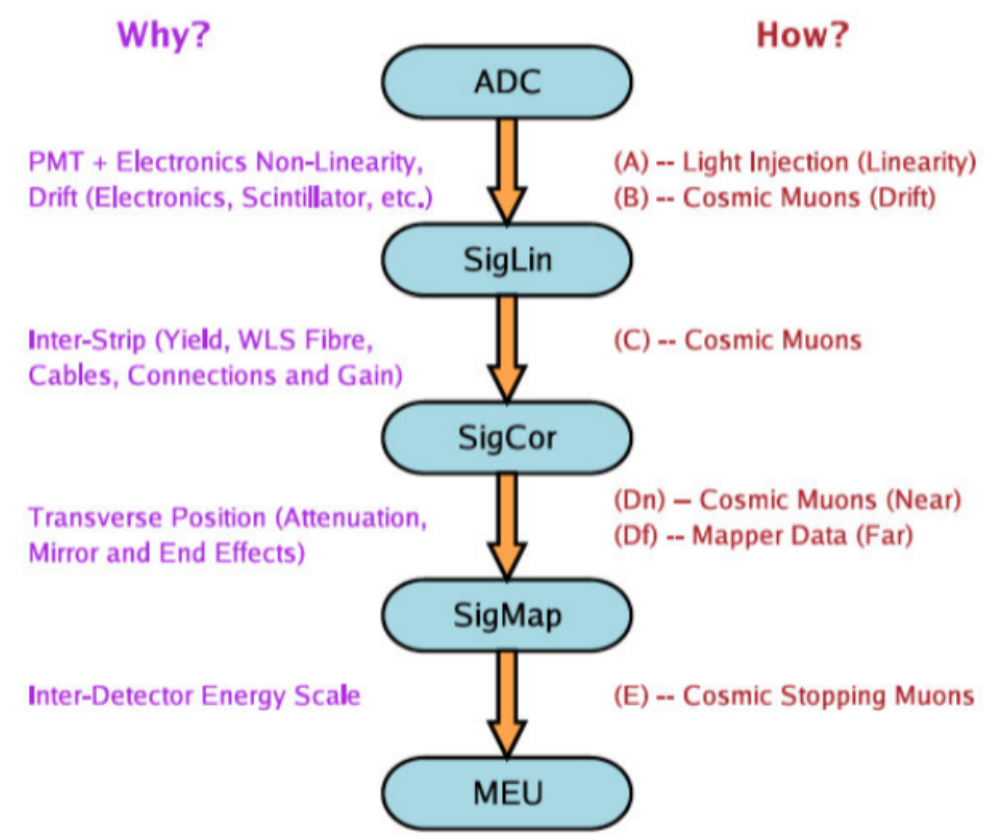

Figure 4.5: Each step corresponds to a correction factor in equation 4.3 . The correction from raw pulse height (ADC) to SigLin applies the linearity calibration, $L$ and drift correction $D$, from SigLin to SigCor the strip-tostrip calibration $S$, from SigCor to SigMap the attenuation correction $A$ and finally the absolute energy scale calibration $M$ to convert the raw signal into the standardised energy unit MEU. Figure is taken from [119]. 



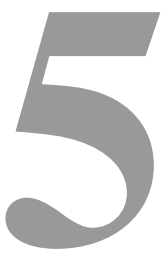

\section{Absolute Energy Calibration of MINOS Detectors}

Absolute energy calibration is the final stage of the MINOS calibration chain. It refers to the calibration of absolute scintillator response to a known energy deposition and this is achieved using a particle of known energy. In the MINOS detector sites we do not have particles of known energy. But we have number of other sources at the detector to achieve it. One method is to use the Michel electron from the muon decay or using the reconstructed $\pi^{0}$ mass and setting their energy response. In MINOS detectors it is not possible to use these particles because of the granularity of the detector is not too good to identify these particles.

Using the MINOS calibration detector we have measured detector response using the beam of particles with known energy from the CERN PS accelerator and the values obtained are then used to tune our Monte Carlo (MC) response [102]. In the MINOS detectors, the absolute calibration of the scintillator response to an energy deposition in $\mathrm{GeV}$ is achieved using the relative calorimetric energy measurements of the stopping muons and the MC simulation. The absolute energy deposition per plane of scintillator in $\mathrm{GeV}$ can be calculated using the energy deposited per plane $(d E / d x)$ information and the known scintillator thickness. The momentum of the muons can also be extracted from the $(d E / d x)$ information.

\subsection{Relative Energy Calibration}

Stopping muons are the standard candle for MINOS which allow us to measure the relative calorimetric energy response accurately. The relative energy calibration is performed by normalising the response of the 
three detectors (Calibration detector, Near and Far detectors). Stopping muons are used, because these are the type of particles that have the most accurately known energy at the MINOS detectors.

In order to use muon for the relative energy calibration it is necessary to know the muon energy accurately since $(d E / d x)$ changes as a function of momentum. The stopping muons with a given track length gives the same response in the MINOS detectors. The determination of muon relative energy calibration by the measuring momentum of muon through it's range measurement is expected to have a relative accuracy of only $2 \%$ between the MINOS detectors. Given that the relative calibration has to be accurate better than $2 \%$, this dependence on the stopping muon range measurement is not acceptable.

The inter-detector calibration in MINOS is expressed in terms of Muon Energy Unit (MEU). To within the errors of the calibration and the scintillator thickness, $1 \mathrm{MEU}$ of measured detector response corresponds to the same quantity of energy deposited in the scintillator at all MINOS detectors. The exact definition of a MEU is given in (Equation. 5.3). As a rule of thumb, $1 \mathrm{MEU}$ is equals to the detector response when a $1 \mathrm{GeV}$ muon traversing perpendicular to one plane of the scintillator.

\subsubsection{Calculation of MEU - Track Window Method}

The track window method is used to calculate the MEU numbers. In this method, we choose the stoping muons track and create a window where $(d E / d x)$ is a constant. For that, it is necessary to consider how the $(d E / d x)$ varies with momentum. The $(d E / d x)$ of a $1.5 \mathrm{GeV}$ muon increases by approximately a factor of two in the last $10 \%$ of its track, where as in the other $90 \%$ of its track the $(d E / d x)$ changes only by approximately $8 \%$. This technique measures the response of muons only when their energy is approximately between $0.5-1.1 \mathrm{GeV}$. The ionization energy loss i.e., $(d E / d x)$ for MINOS detectors is described by,

$$
-\frac{d E}{d x}=K z^{2} \frac{Z}{A} \frac{1}{\beta^{2}}\left[\frac{1}{2} \ln \frac{2 m_{e} e^{2} \beta^{2} \gamma^{2} T_{\max }}{I^{2}}-\beta^{2}-\frac{\delta}{2}\right],
$$

where $Z e, \beta c$ and $\gamma$ respectively are the charge, velocity and relativistic gamma factor of the incident particle. $T_{\max }$ is the maximum kinetic energy which can be imparted to a free electron in a single collision and $Z / A$, 
$A$ and $\delta$ respectively are the ratio of the charge number to the mass number, mean excitation energy and density effect correction for the material traversed by the incident particle. The constant $K=4 \pi N_{A} r_{e}^{2} m_{e} c^{2}$, where $r_{e}$ is the classical electron radius, $N_{A}$ the Avogadro number and $m_{e} c^{2}$ is the rest energy of the electron.

The size of track window used to do the MEU calibration is $83 \mathrm{~cm}$ wide (or 14 planes traversed perpendicularly, i.e, $14 \times 5.94=83 \mathrm{~cm}$ of material) and the window is positioned $95 \mathrm{~cm}$ (16 planes traversed perpendicularly, i.e. $16 \times 5.94=95 \mathrm{~cm}$ of material) from where the muon is stopped, this is represented in Fig. 5.1. Once the end position is calculated we can sum over the planes in the window and calculate the MEU using the relation,

$$
M E U=\frac{1}{N_{p}} \sum_{n=1}^{N_{p}} \frac{S_{i}}{L_{i}}
$$

where $S_{i}$ is the detector response (signal in intra detector calibrated unit), $L_{i}$ is the path length in the plane and it is summed over all the planes $\left(N_{p}\right)$ in the track window, in general $N_{p}<14$ planes (depends upon the steepness of the track). The above equation is the response to one single muon. In order to avoid the statistical fluctuations and to get a correct estimate of the MEU, it is calculated using a large number of muons $(N)$ and the possible centroid is obtained, where the centroid are mean and median of the distribution. Here the MEU is taken as the median of the distribution, i.e.,

$$
M E U=\operatorname{Median}\left(\left(\frac{1}{N_{p}} \sum_{n=1}^{N_{p}} \frac{S_{i}}{L_{i}}\right)_{1},\left(\frac{1}{N_{p}} \sum_{n=1}^{N_{p}} \frac{S_{i}}{L_{i}}\right)_{2}, \ldots,\left(\frac{1}{N_{p}} \sum_{n=1}^{N_{p}} \frac{S_{i}}{L_{i}}\right)_{N}\right)
$$


Track Window Position

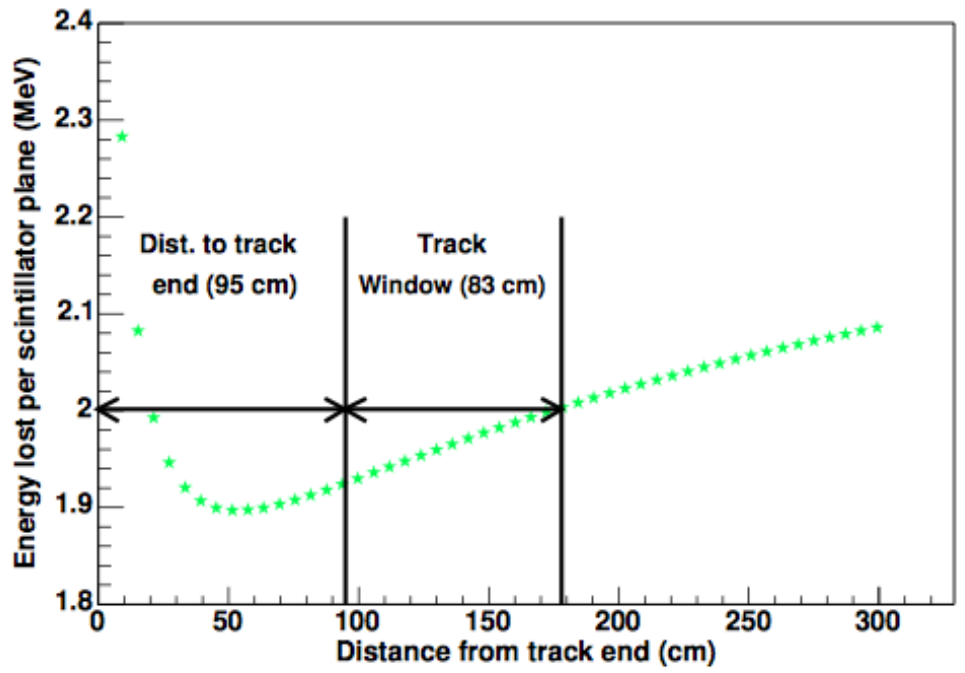

Figure 5.1: The track window used for the MEU calibration

\subsubsection{Far Detector Performance Over Time}

The MINOS Far detector has started operation in 2003 and for the last several years we have been taking data, during this time it is very likely to be a change in the detector response because of various reasons. So, it is useful to examine the MEU as a function of time to ensure that the calibration procedure is stable and able to account for the aging effects of the detector components. In the case of Far Detector we use only cosmic muons for the MEU calculation since the beam muon statistics is not enough to calculate the MEU. The time dependence of the FD MEU is shown in the Fig 5.2 and from that we can see that the MEU is stable to within $1.5 \%$. The plots shows the stability for the new MINOS+ run era starting from September 2013. 

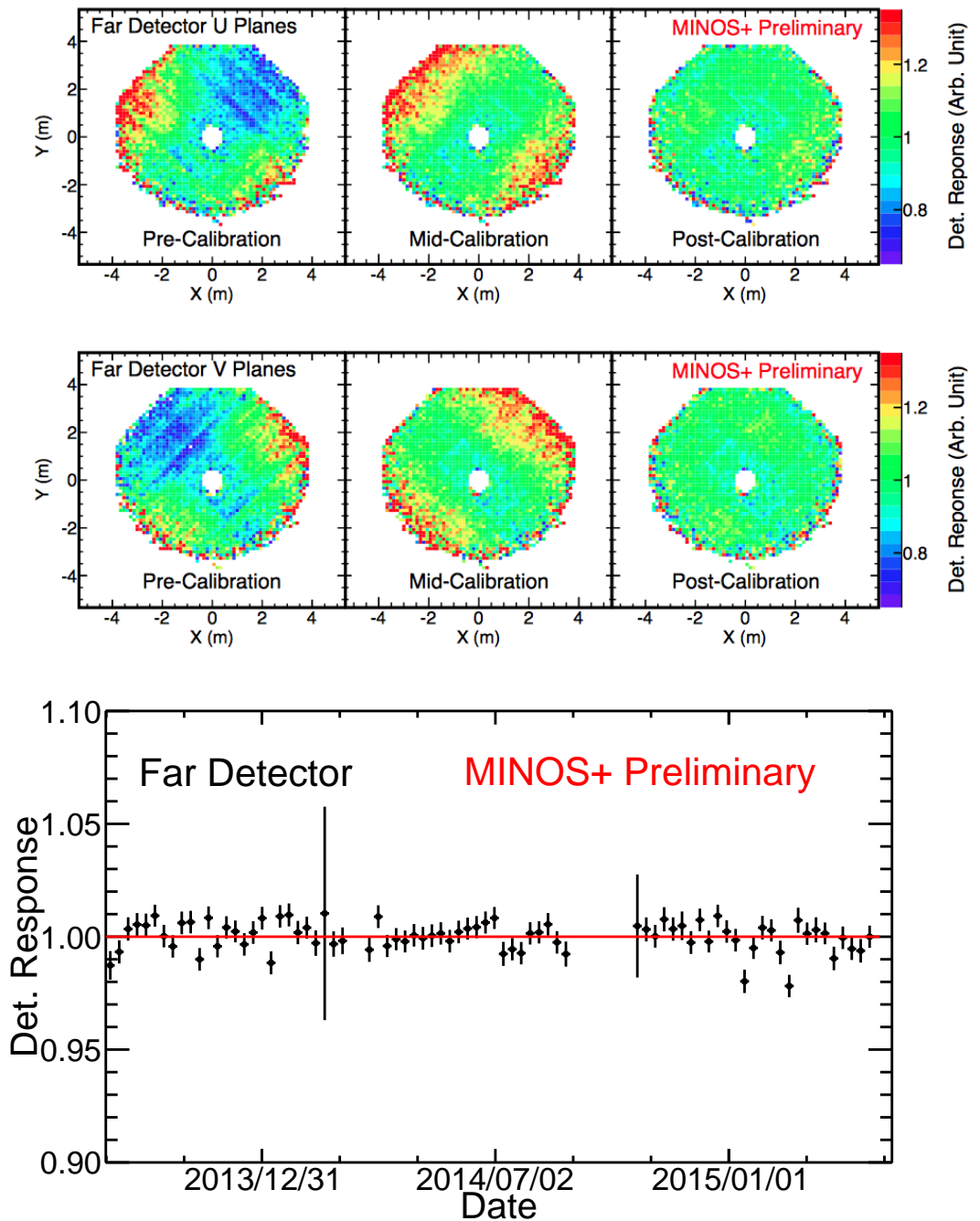

Figure 5.2: The detector response over the time, the plots show the stability of the Far Detector in the MINOS+ era and it is with in 1\%. Each data point corresponds to 1 week period of data. 


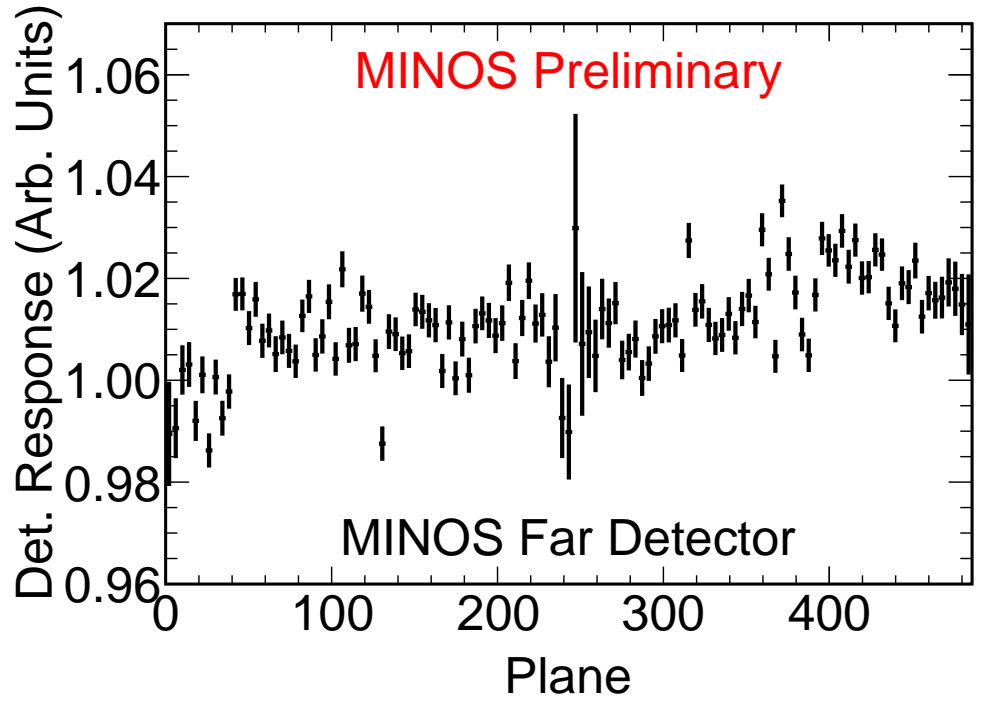

Figure 5.3: Far Detector MEU as a function of plane number, as measured using cosmic data. Individual runs outside of the run period boundaries were removed.

\subsubsection{Near Detector Performance Over Time}

In the Near Detector we can use both beam and cosmic muons for the MEU calculation. The response is stable with in $0.5 \%$. Beam muons and cosmic muons are used to find the absolute energy response. The Near Detector started operation in 2005. 

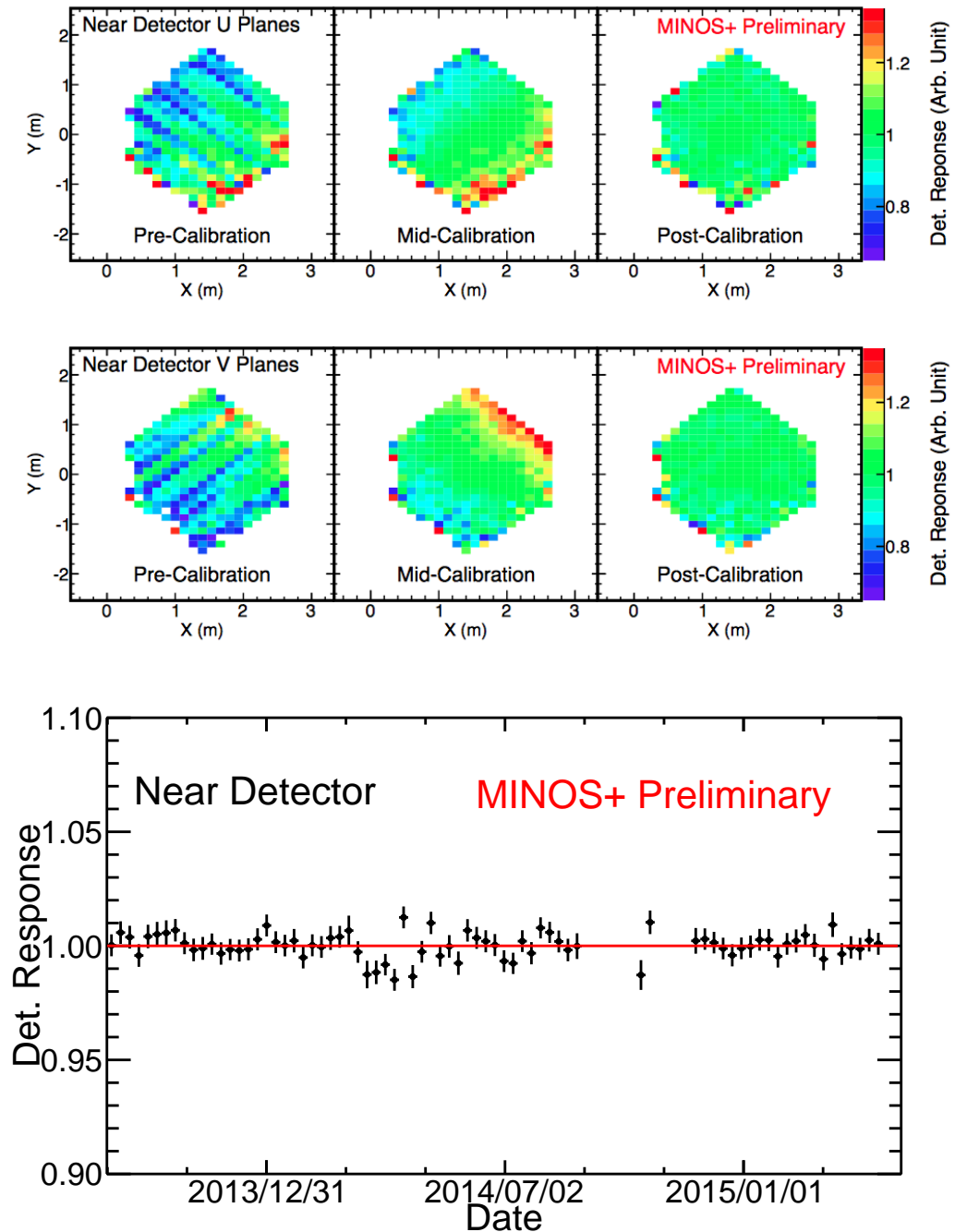

Figure 5.4: The detector response over the time, the plots show the stability of the Near Detector in the MINOS+ era, and it is stable with in 1.5\%. Each data point corresponds to 1 week period of data. 


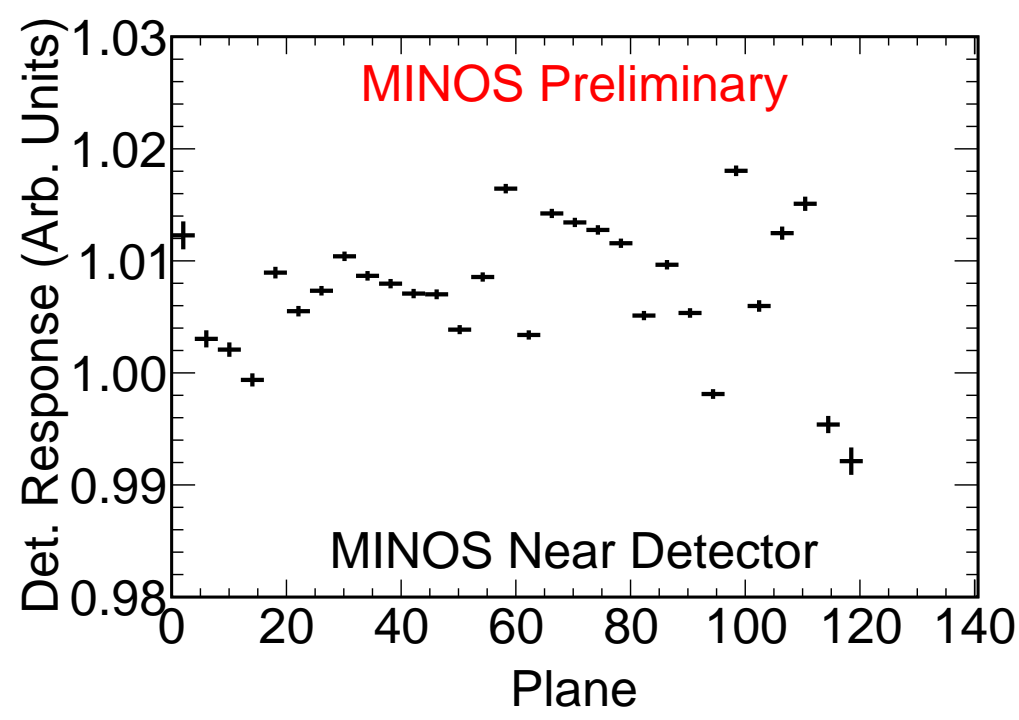

Figure 5.5: Near Detector MEU as a function of plane number, as measured using cosmic data. Individual runs outside of the run period boundaries were removed.

\subsubsection{Angular Dependence on Near and Far Detec- tor}

The MEU has a dependence on the zenith angle of the track this is shown in Fig. 5.6. As can be seen, the dependence is reasonably well modeled by the MC. To reduce uncertainties in the calibration due to this dependence, only muon tracks with $\cos \theta_{z}>0.3$ are used for the MEU calibration. After applying the angular correction it can be seen that the response became flattened or independent of the zenith angle. 

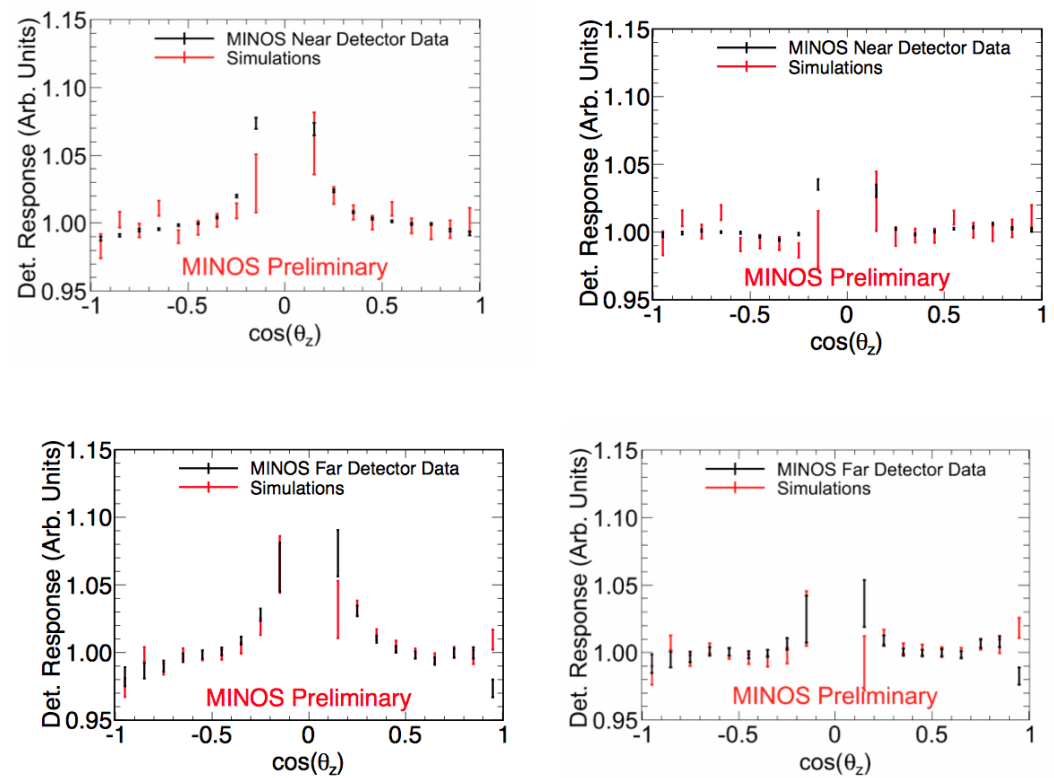

Figure 5.6: Detector response per plane to muon hits in the track window as a function of track zenith angle. Angular correction has been applied to remove the $\cos \theta_{z}$ dependence on detector response, and the distribution gets flattened after applying the angular correction.

\subsection{MEU Systematics}

The sources of systematic error considered for this calibration study are spatial variation, Data-Monte Carlo difference and the MEU differences between the cosmic and spill stopping muon. By default near and Far Detector MEU calculation utilizes stopping muons extracted from the cosmic muon data set. The current statistical precision is around $0.05 \%$ in ND and FD. The light level is tuned in the cosmic muon Monte Carlo to give a MEU similar to that of the data. The level of agreement to the data is dependent on how the MEU is evaluated. Under the normal approach defined in Equation. 5.3 the difference between data and $\mathrm{MC}$ is only a few percentage for the near and Far Detector. Different techniques to extract the centroid of the distribution of an ensemble of stopping muon events differ between data and Monte Carlo by $0.05 \%$. 
The calibration chain is designed to remove spatial and temporal variations in the MEU. The cosmic stopping muon statistics are large enough that the fiducial volume can be broken down to identify spatial biases across the detector. For this study the fiducial volume of the near and Far Detectors have been broken into concentric rings of equal fiducial mass centered around the beam spot and in four volumes parallel to the beam. The detector response was found to be more uniform along the z-axis and the maximum deviation in the concentric rings was $0.73 \%$ in the Near Detector and $0.76 \%$ in the Far Detector.

The error arises due to difference in MEU between the spill and cosmic stopping muons in the data samples are also considered as a systematic. In section 5.1.4 an additional path-length correction was introduced to reduce the impact of the radiative component of the muon energy loss. Even after this correction it was noted that the spill-cosmic MEU differs by $0.5 \%$ in the Near Detector and in the Far Detector we do not have enough beam muons to compare it. This is a concern as the calibrations are derived using cosmic muons, but we apply the calibrations to beam muons. There are other differences between the cosmic and spill data samples beyond just the angular correction. The cosmic sample is typically contains positive and negative muons. It has equal probability to be going forward or backward. The beam muons come from the NuMI beam are all forward going and are predominantly negatively charged muons in neutrino mode. The cosmic sample shows no bias in the MEU when calculated using forward or backward going muons, however, it was observed that the MEU for positive muons was approximately $0.2 \%$ larger than it is for negative muons. This observation is consistent with the previous muon range measurements study [103].

\subsection{Intensity effect on Beam MEU}

MEU is calculated using the cosmic muons, however, the beam muons are also used in the calculation of MEU and then it is compared with the cosmic MEU. The cosmic muons do not cause pile up effect thus we can see one track at a time, but for the beam muons many events can be occurred in the $10 \mu s$ spill window, we have studied the effect of intensity on the MEU numbers. This studies has been done in both MINOS as well as the MINOS+ era samples. 
Table 5.1: MEU systematics table

\begin{tabular}{ccc}
\hline \hline Systematics & Near Detector & Far Detector \\
\hline Data/MC difference & $0.9 \%$ & $0.2 \%$ \\
Beam/Cosmic difference & $0.05 \%$ & - \\
Spatial variations & $0.22 \%$ & $0.84 \%$ \\
Forward /Backward & $0.30 \%$ & $0.04 \%$ \\
$\mu^{+} / \mu^{-}$ & $0.4 \%$ & $0.6 \%$ \\
\hline
\end{tabular}

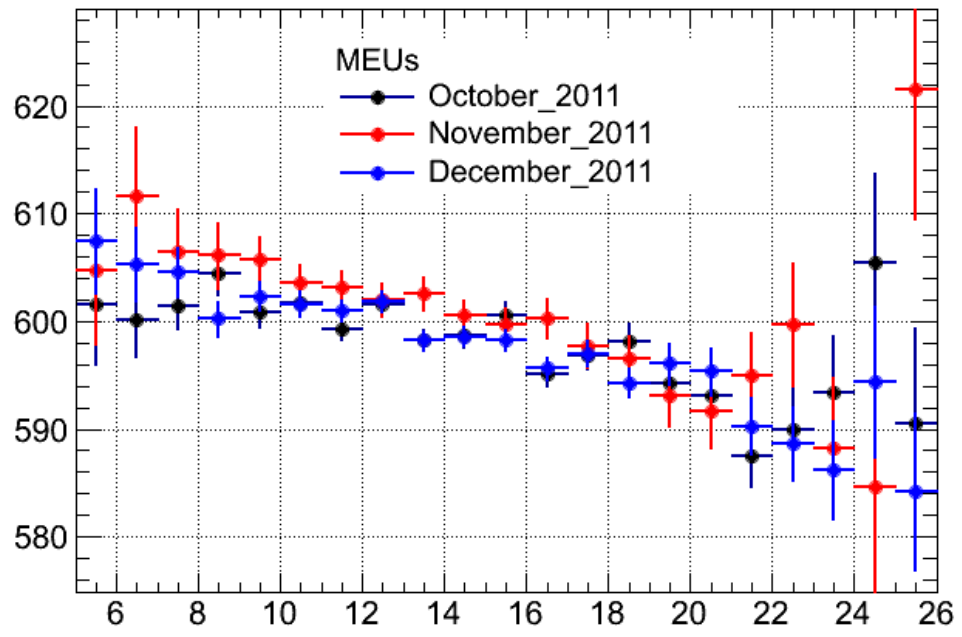

Figure 5.7: MEU as a function of number of events in a snarl, in the MINOS Near Detector. It is clear that as there are more events in the snarl, MEU drops. This is studied by splitting the data set into different month.

In MINOS + era, we have improved our reconstruction in order to deal with the medium energy neutrino beam, the algorithms were improved to take into account the pile up and event merging effects. The intensity 
dependence on the MEU value is studied, there is no a clear evidence that the MEU is decreasing as intensity increases.

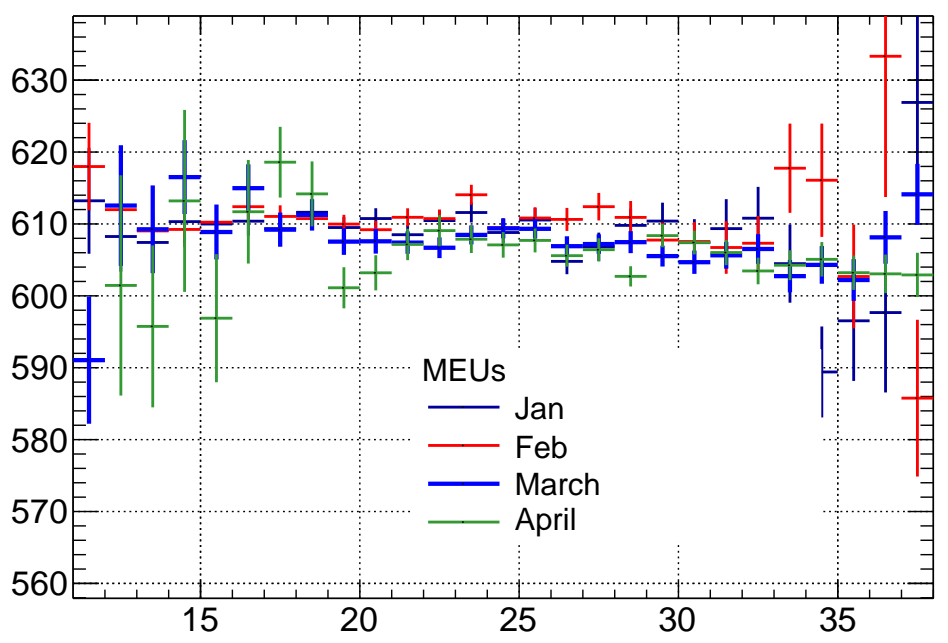

Figure 5.8: MEU as a function of number of events in a snarl, in the MINOS + era. No clear evidence that MEU is decreasing. This is studied by splitting the data set into different month.

\subsection{Need of Relative Energy Calibration}

In order to measure the oscillation parameters $\left(\Delta m_{23}^{2}, \sin ^{2}\left(2 \theta_{23}\right)\right)$ accurately a precise energy measurement is needed. The reconstructed $v_{\mu}$ $\mathrm{CC}$ event energy is estimated as the sum of the muon energy and hadronic shower energy deposited in the detector. The true energy of the interaction is given by

$$
E_{v_{\mu}}^{\text {true }}=E_{\mu}^{\text {vis }}+E_{\text {hadronic }}^{\text {vis }}+E^{\text {invisible }}
$$

where $E_{v_{\mu}}^{\text {true }}$ is the true energy of interaction, $E_{\mu}^{\text {vis }}$ is the visible muon energy $E_{\text {hadronic }}^{\text {vis }}$ is the visible hadronic shower energy and $E^{\text {invisible }}$ invisible energy, that is the energy absorbed by the nucleus. The visible energy itself fluctuates significantly in each event, it can be due to the unmeasurable fluctuation of energy loss occurring at the passive detector components and also due to the fluctuations in the light amount passing through the 
optical read out system. So the reconstructed energy of the neutrino event can be significantly different from that of the true energy.

The muon energy resolution is estimated as,

$$
\frac{\sigma_{\mu}}{E_{\gamma_{\mu}}}=6 \%
$$

where $E_{v_{\mu}}$ is the energy in $\mathrm{GeV}$. The hadronic energy resolution is given by,

$$
\frac{\sigma_{\text {hadronic }}}{E_{\text {hadronic }}}=\frac{56 \%}{\sqrt{E_{\text {hadronic }}}}
$$

where, $E_{\text {hadronic }}$ is also measured in $\mathrm{GeV}$. In addition to this smearing, there can be relative energy scale offset between near and Far Detector in hadronic energy, given by

$$
E_{\text {hadronic }}^{\text {near }}=(1+\epsilon) E_{\text {hadronic }}^{\text {far }}
$$

$E_{\text {hadronic }}^{\text {near }}$ and $E_{\text {hadronic }}^{\text {far }}$ are the measured calorimetric energy in near and Far Detector and $\epsilon$ can be treated as the shift in the calorimetric energy scale due to the calibration error. A $10 \%$ shift in the energy scale can affect the energy spectra, hence the oscillation parameters. The Fig. 5.9 shows the shift in the energy for a $10 \%$ shift in the energy scale. A detailed description is given in reference [104]. 


\section{Neutrino Visible Energy Spectra}

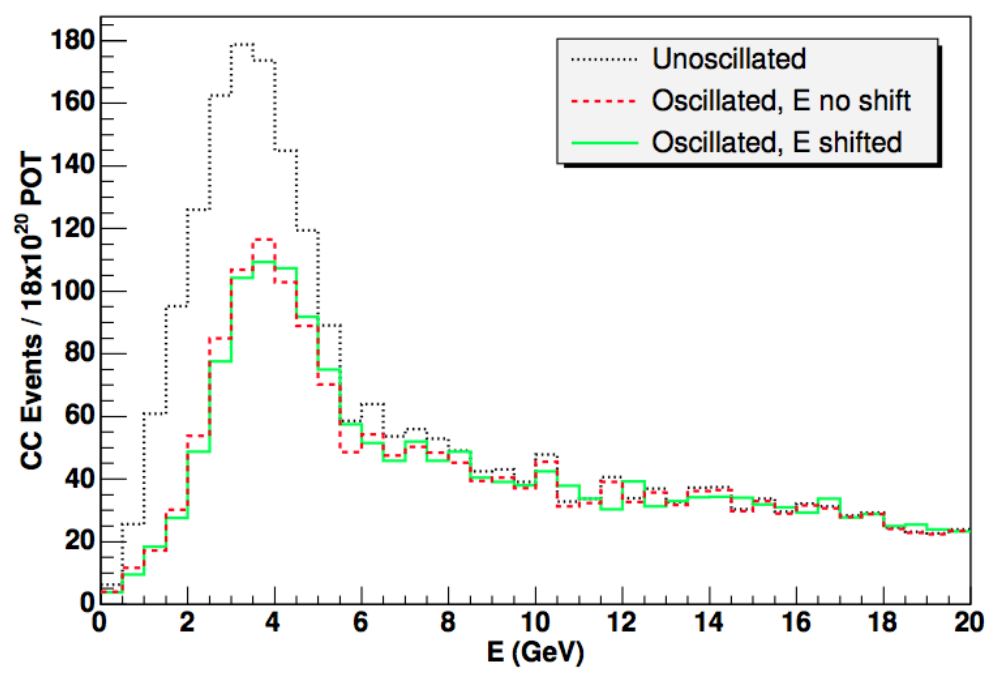

Figure 5.9: The black dotted line shows the unoscillated energy spectra, and the green and red are oscillated spectra with oscillation parameter $\Delta m_{23}^{2}=0.0025 \mathrm{eV}^{2}$ and $\sin ^{2} \theta_{23}=0.95$, with $10 \%$ shift and with out shift respectively.

Due to this energy scale shift there can be fractional bias on $\Delta m_{23}^{2}$ and $\sin ^{2} 2 \theta_{23}$, the fractional bias is defined as

$$
\begin{gathered}
B\left(\Delta m^{2}\right)=\frac{\Delta m_{\text {fit }}^{2}-\Delta m_{\text {true }}^{2}}{\Delta m_{\text {true }}^{2}} \\
B\left(\sin ^{2}(2 \theta)\right)=\frac{\sin ^{2}(2 \theta)_{\text {fit }}-\sin ^{2}(2 \theta)_{\text {true }}}{\sin ^{2}(2 \theta)_{\text {true }}}
\end{gathered}
$$

where fit means the value obtained from the fitting and true means once which obtained from MC truth information. 


\section{Fractional $\Delta \mathbf{m}^{2}$ Bias vs. Energy Shift}

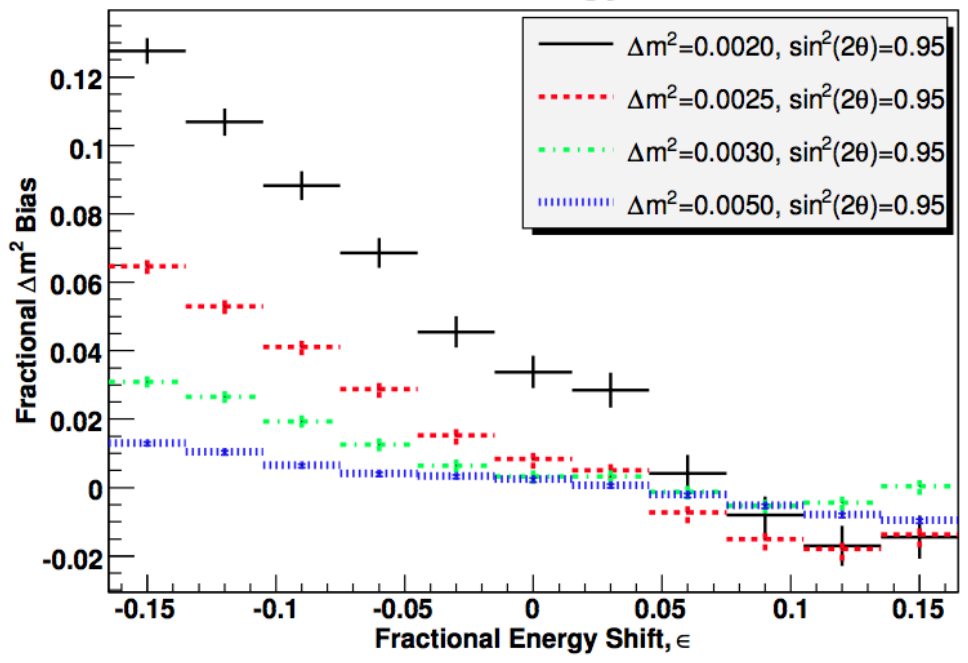

Figure 5.10: Fractional bias for $\Delta m^{2}$ for the spectrum which oscillated with values of $\Delta m^{2}$ and fixed $\theta$.

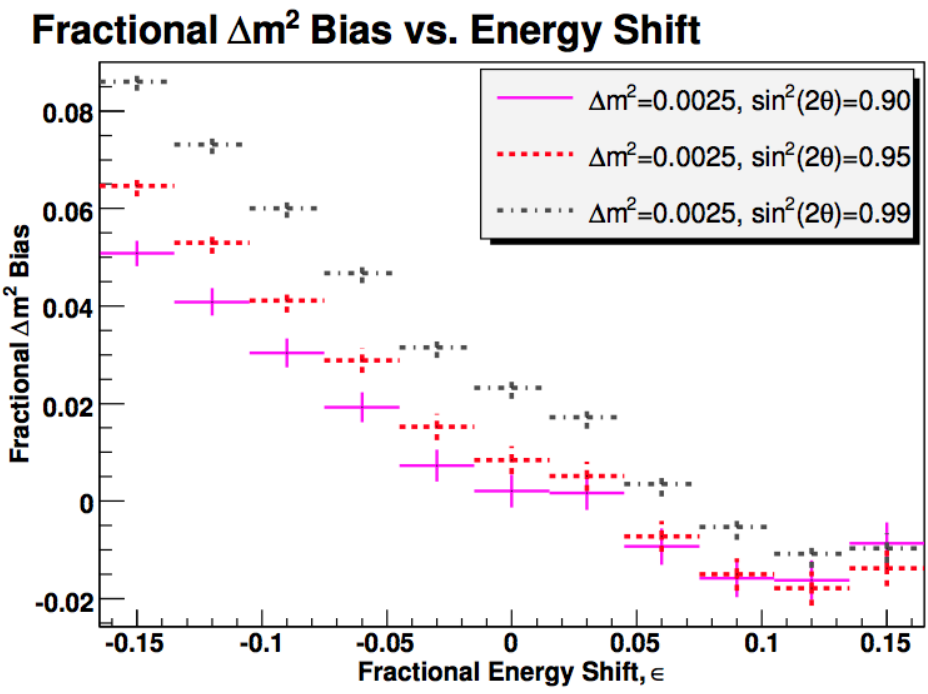

Figure 5.11: Fractional bias for $\Delta m^{2}$ for the spectrum which oscillated with a fixed value of $\Delta m^{2}$ and different values of $\theta$. 


\subsection{MEU Charge Sign Analysis}

An experiment at LBNL measured a $0.2 \%$ difference in the ranges of positive and negative muons [105]. The experiment uses the muons coming out from the kaon decay with different momenta varying between 500 and $1500 \mathrm{MeV} / \mathrm{c}$. It is observed that the range of the muon with the positive and negative tracks have a difference of $0.2 \%$. This could be explained by modifying the Mott scattering to include terms of order $e^{6}$, a correction term of $( \pm) \pi \alpha \beta$ added to Bethe-Bloch to take into account for the energy loss, where $\alpha$ is the fine structure constant which would be $(+)$ for positive muons and $(-)$ for negative muons, which is responsible for this effect. We also have a good collection of cosmic and beam muons in MINOS ND, so we can test this effect for $\mu^{+}$and $\mu^{-}$in different magnetic field configuration and direction of the muons.

In MINOS to do this study, instead of measuring the range we measure the MEU. Range measurement is not used here, because of the high uncertainty of measuring the range, around $2 \%$. The muon energy loss is given by,

$$
-\frac{1}{\rho} \frac{d E}{d x}=\frac{2 \pi n e^{4}}{m c^{2} \rho \beta^{2}}\left\{\ln \left(\frac{2 m p^{2} W}{I^{2} \mu^{2}}\right)-2 \beta^{2}-\delta-U\right\},
$$

where $E$ is the energy of the particle, $p$ is its momentum and $\mu$ is its mass. The dependence on the momentum is removed by choosing the track window which we use for the MEU calculation and this ensures that energy of the particle moving in the window changes in a slow rate.

We compare the MEU values for cosmic and beam muons at ND including all the stopping muons. We also calculate MEU values for positive and negative muons individually, as well as with the magnetic field forward and reversed, for muons that are forward and backward going and for various combinations of these.

To select the negative and positive muons in the MEU sample and to make the sample more pure we apply a charge sign selection cut. The cut applied is,

$$
\frac{q / p}{\sigma(q / p)}
$$

We require $\frac{q / p}{\sigma(q / p)}>1$ for positive muons track and $\frac{q / p}{\sigma(q / p)}<1$ for negative muon track. In order to determine if there is any difference in the energy 
loss between positive and negative muons, we calculate a ratio,

$$
R_{x}=\frac{M E U_{x}^{+}}{M E U_{x}^{-}}
$$

where $M E U_{x}^{ \pm}$is the MEU value for $\mu^{+}\left(\mu^{-}\right)$for a given configuration $x$, for example, forward-field (FF) forward-going (FG), reversed-field (RF) backward-going (BG). The ratios for various configurations are shown in the table.

Table 5.2: MEU charge sign study table

\begin{tabular}{cc}
\hline \hline Ratio & ND cosmic data \\
\hline$R_{A l l}$ & $1.002934 \pm 0.000149$ \\
$R_{F F}$ & $1.004479 \pm 0.000176$ \\
$R_{R F}$ & $0.999073 \pm 0.000276$ \\
$R_{F F F G}$ & $1.000867 \pm 0.000292$ \\
$R_{R F B G}$ & $1.002806 \pm 0.000416$ \\
$R_{R F F G}$ & $1.002940 \pm 0.000383$ \\
$R_{F F B G}$ & $1.001486 \pm 0.000277$ \\
\hline
\end{tabular}

This tables shows the ratio $R_{x}$ for different field configuration and direction. Statistical uncertainties on MEU values are determined by taking the error on the median. In order to estimate the overall difference, we combine the ratios by taking the error-weighted average. Statistical uncertainties are combined in a similar manner and the systematic uncertainty is estimated by taking the standard deviation of the ratios. For the purposes of these calculations only the four ratios in the bottom of the table are considered.
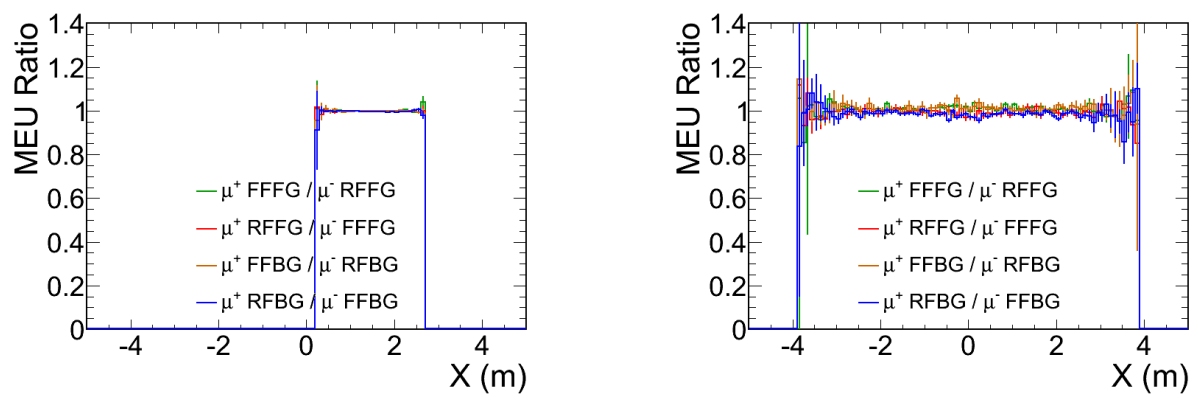

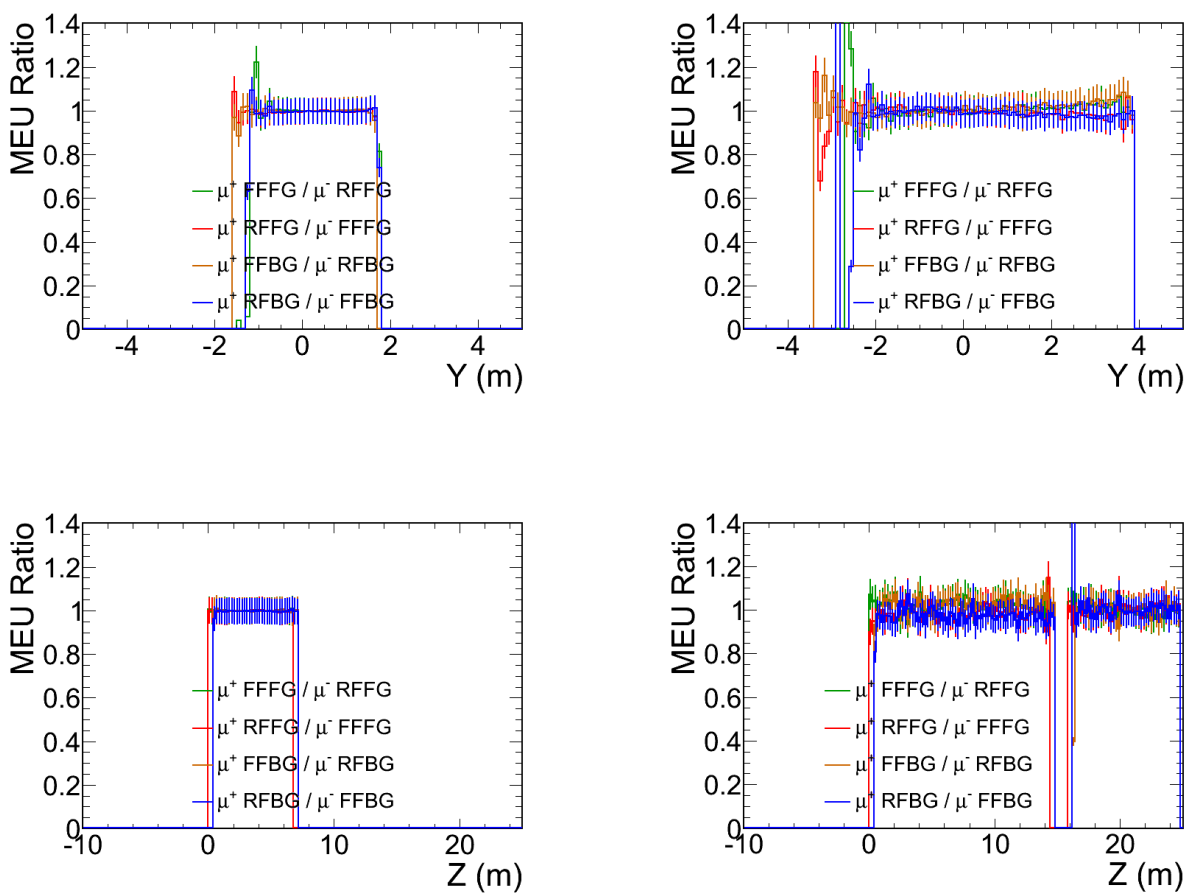

Figure 5.12: The ratio of MEU for different configuration as a function of X,Y and Z hit. Near Detector distribution (left) and Far Detector distribution (right).

The resulting ratio is given as $\mathrm{R}=1.00227 \pm 0.00013$ (stat) \pm 0.00063 (syst), or $\mathrm{R}=1.00227 \pm 0.00065$ (total). This translates to a $0.23 \%$ difference between positive and negative muon MEU values, consistent with the 1972 LBNL experiment. The positive muons have a larger MEU than negative muons.

\subsection{Summary and Conclusion}

MINOS experiment measures the neutrino oscillation parameters, $\Delta m_{23}^{2}$ and $\sin ^{2} \theta_{23}$ by comparing neutrino energy spectrum at near and Far Detector. This is achieved by measuring the calorimetric energy. The relative energy scale calibration between the Near and Far detectors is very important since we measure the oscillation parameters by comparing the neutrino energy spectrum at both detectors. The relative energy scale calibration is done using stopping muons at both detectors. Various factors 
affecting this particular stage of calibration is studied, this includes the angular correction which makes the beam and cosmic muon calibration identical, the effect of high intensity and the reconstruction algorithm. A charge sign analysis is also performed and the measured energy response of muons and anti-muons is found to be consistent with the theory. 



\section{6 \\ Sterile Neutrino Search using Antineutrinos}

This chapter describes the sterile neutrino analysis performed on MINOS $\bar{v}_{\mu}$ mode run corresponding to a Proton on Target (POT) of $3.36 \times$ $10^{20}$. In this beam configuration the neutrino beam components are $39 \% \bar{v}_{\mu}, 59 \% v_{\mu}$ and $2 \%\left(v_{e}+\bar{v}_{e}\right)$. MINOS has unique capability of putting limit on sterile neutrino parameter space over a large region of $\Delta m_{41}^{2}$ ranging from $10^{-4}$ to $10^{2} \mathrm{eV}^{2}$. The analysis uses covariance matrix as a tool to incorporate the energy dependent systematics in the fit and this is different from the conventional $\chi^{2}$ method that MINOS uses [106]. Here we use a $\chi^{2}$ fit to the Far over Near ratio of the reconstructed energy, assuming a Gaussian probability distribution.

\subsection{Sterile Neutrinos}

Sterile neutrino searches have been became a major study of many ongoing neutrino experiments, if they exist it can have many potential physics implications. For example, in cosmology sterile neutrinos are important candidates in many models. There has been evidence for neutrino oscillations for $\Delta m^{2}=1 \mathrm{eV}^{2}$ in the recent experiment like LSND [58] and MiniBooNE [66] and this anomaly has not been fully resolved yet. In MINOS, we study the simple $3+1$ model, where one additional state is added to the standard three flavor scenario. In order to describe the four flavor oscillation, we need additional mass squared splitting, mixing angles and phases. 


\subsection{Sterile Neutrinos in MINOS}

The $3+1$ oscillation probability for $v_{\mu} \rightarrow v_{\mu}$ with an additional neutrino flavor state can be written as [107,

$$
P\left(v_{\mu} \rightarrow v_{\mu}\right)=1-4\left|U_{\alpha 4}\right|^{2}\left(1-\left|U_{\alpha 4}\right|^{2}\right) \sin ^{2} \Delta_{41},
$$

where $\Delta_{41}=\Delta m_{41}^{2} / 4 E$. Considering the results from the short baselines neutrino experiment, i.e, $\left|U_{e 4}\right|^{2}=\sin ^{2} \theta_{14} \ll 1$ and $\left|U_{\mu 4}\right|^{2}=$ $\cos ^{2} \theta_{14} \sin ^{2} \theta_{24} \ll 1$, and $\Delta m_{32}^{2} / 4 E \leq 1$, we can expand the $v_{\mu}$ survival probability in MINOS to second order in the small angles $\theta_{13}, \theta_{14}, \theta_{24}$ and $\left(\theta_{23}-\pi / 4\right)$ to get, [107],

$$
P\left(v_{\mu} \rightarrow v_{\mu}\right)=1-\sin ^{2}\left(2 \theta_{23}\right) \cos \left(2 \theta_{24}\right) \sin ^{2} \Delta_{32}-\sin ^{2}\left(2 \theta_{24}\right) \sin ^{2} \Delta_{41}
$$

Similarly we can write it for $v_{\mu} \rightarrow v_{s}$ to first order in small angles to get, [107],

$$
\begin{aligned}
1-P\left(\nu_{\mu} \rightarrow \nu_{s}\right) & =1-\cos \theta_{14}^{2} \cos \theta_{34}^{2} \sin ^{2}\left(\Delta m_{41}^{2}\right) \\
- & \sin ^{2}\left(\theta_{34}\right) \sin ^{2}\left(2 \theta_{23}\right) \sin ^{2} \Delta_{31}^{2} \\
- & 1 / 2 \sin \left(\delta_{24}\right) \cos \left(\theta_{24}\right) \sin 2\left(\theta_{34}\right) \sin 2\left(\theta_{23}\right)
\end{aligned}
$$
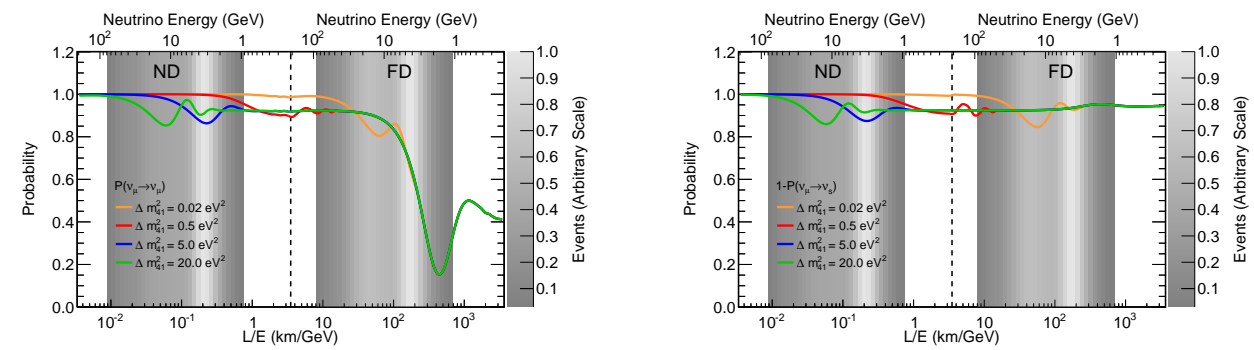

Figure 6.1: The probability as a function of $L / E$ for charged current (left) and neutral current events (right) for different values of $\Delta m_{41}^{2}$, the position of detector is also shown.

Depending upon the mass squared splitting the oscillation can happen in one of the detectors or in both detector together. For $10^{-3} \mathrm{eV}^{2} \lesssim$ $\Delta m_{41}^{2} \lesssim 0.1 \mathrm{eV}^{2}$, there would be an energy dependent depletion at the 
Far Detector and no effect at the Near Detector. In the region where $\Delta m_{41}^{2} \approx 1 \mathrm{eV}^{2}, \Delta_{41} \gg \pi / 2$, at Far Detector a reduction in the event rate would be observed as $\sin ^{2} \Delta_{41} \rightarrow 1 / 2$, when the finite resolution of the detector is considered. As $\Delta m_{41}^{2}$ increases further, above $\Delta m_{41}^{2}>1 \mathrm{eV}^{2}$ the oscillation start affecting the Near Detector also and causing more structure in the exclusion region. All the effects are shown in Fig 6.1. For the smaller value of $\Delta m_{41}^{2}$ (below $10^{-3} \mathrm{eV}^{2}$ ) the sterile neutrino oscillation dip lies in the same energy region as the atmospheric oscillation dip, and becomes a degenerate region. The neutral current samples are mainly sensitive to the angle $\theta_{34}, \delta_{24}$ in addition to $\theta_{24}$. The $\mathrm{CP}$ violating phase has very small impact in the $\mathrm{CC} \boldsymbol{v}_{\mu}$ disappearance, see Fig 6.2 . The sensitivity from neutral current samples are limited by poor energy resolution. We included only charged current sample in this analysis.
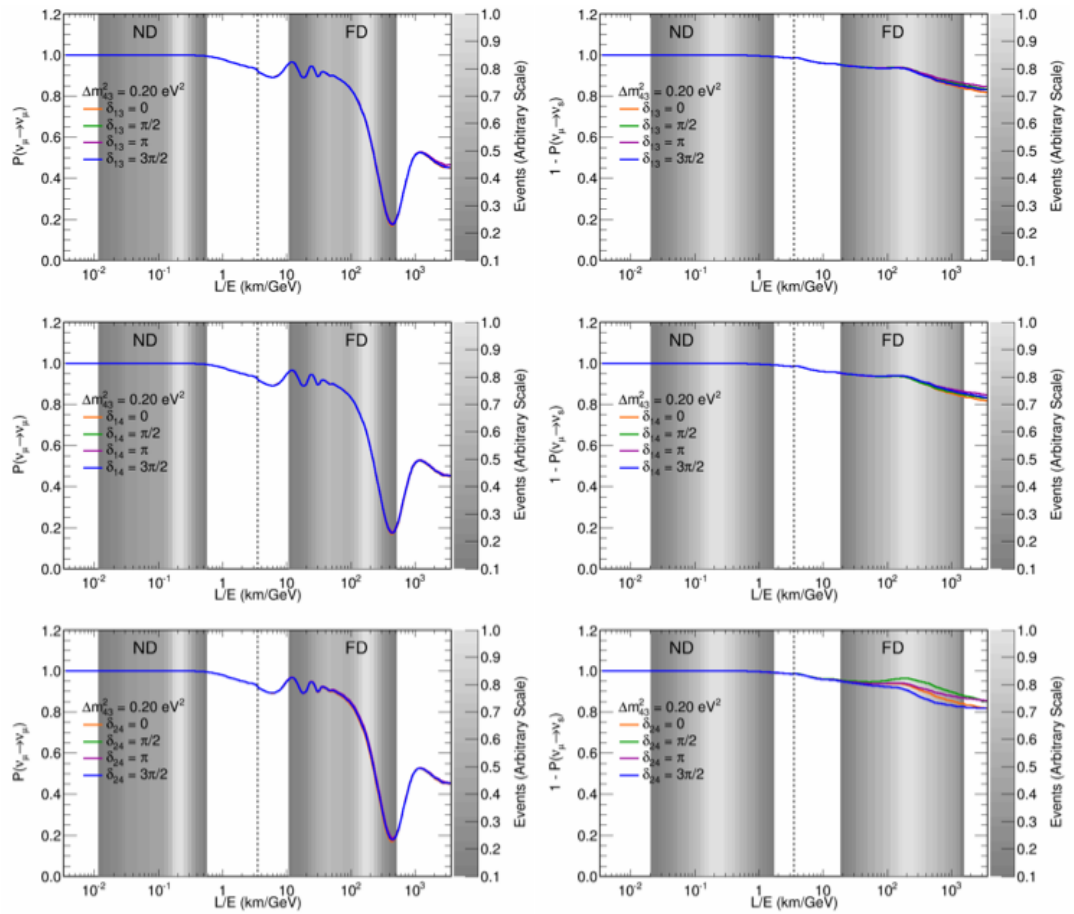

Figure 6.2: Shows the probability as a function of L/E. The effect of CP violating phase for $v_{\mu}$ disappearance and $v_{\mu} \rightarrow v_{s}$. For $v_{\mu}$ disappearance the effect is negligible and there is a small effect in the $v_{\mu} \rightarrow v_{s}$. We ignore these small effects. 


\subsection{Data Stability and Preselection of An- tineutrino Events}

MINOS has taken data in $\bar{v}_{\mu}$ enhanced mode during the operated runs between 2009 and 2011 and collected $3.36 \times 10^{20}$ POT of data. These includes Run IV, Run VII and Run IX data. In order to select pure $\bar{v}_{\mu}$ many requirements are necessary. Primary requirement is that the data quality of the collected events, that is only events collected in good operation periods of running are taken. The good operation periods are related to the proper horn and the magnetic field configurations in the MINOS beam configuration. The data-quality plot is shown Fig 6.3.

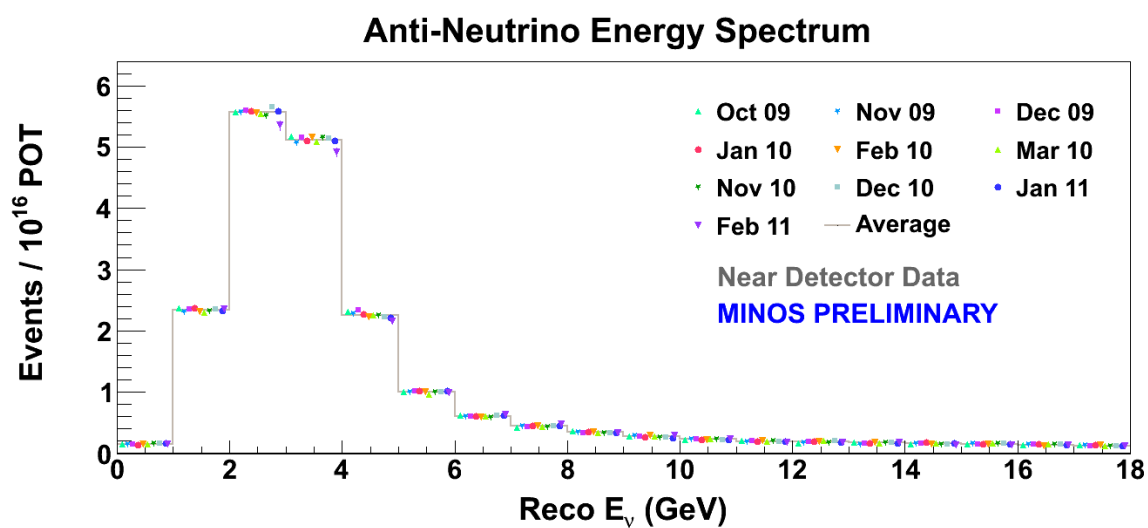

Figure 6.3: Number of charged current anti-neutrino events per $10^{16}$ protons-on-target(POT) as a function of reconstructed neutrino energy in $\mathrm{GeV}$. The data are subdivided into calendar months. 

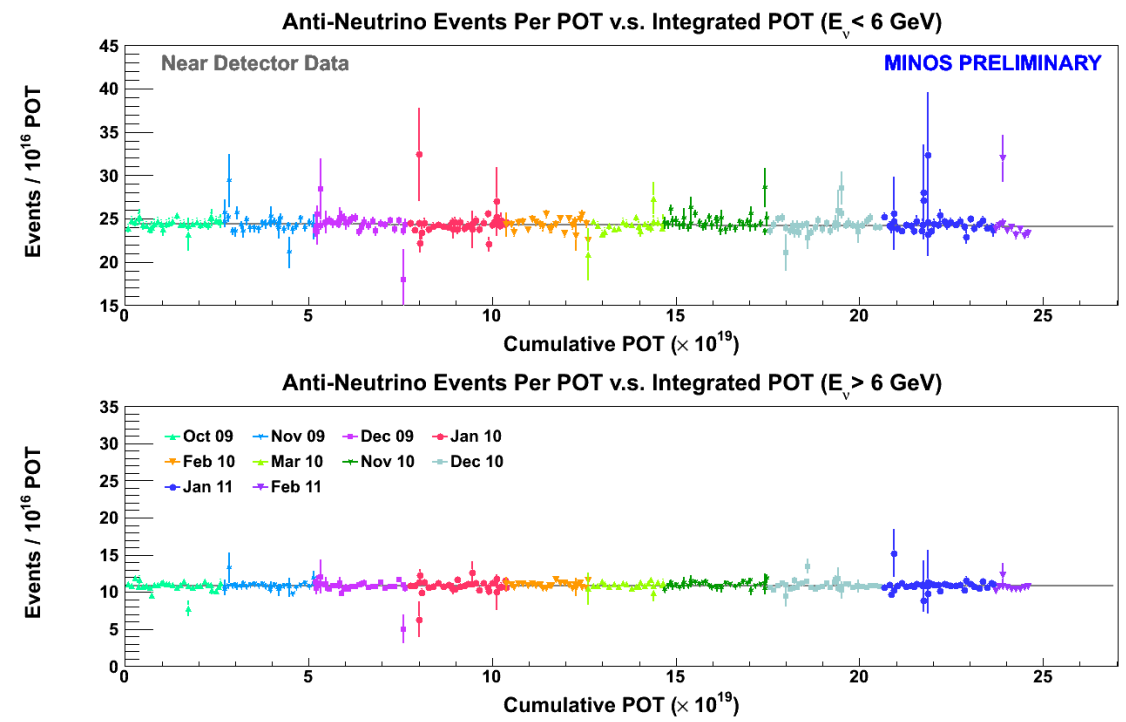

Figure 6.4: Number of charged current muon antineutrino events per $10^{16}$ protons-on-target as a function of total integrated exposure. The data are shown for two energy ranges, $<6 \mathrm{GeV}$ and $>6 \mathrm{GeV}$ and are subdivided into calendar months.

We also have to suppress the atmospheric neutrino background from our beam neutrino events, to do so, all the events selected should be with in a window of $[-2 \mu s, 12 \mu s]$ respectively to the nearest spill of the NuMI beam. These atmospheric events can be identified by looking at the direction of the angle between the track and the beams, because more vertical events are always atmospheric neutrino events, $\cos \theta_{z}>0.6$ is used to get the beam data events at the Far Detector. It is also necessary to make sure that the selected events are completely reconstructed and track vertices are completely within the detector, that is inside the fiducial volume of the detector. The fiducial volume definition for Near Detector and Far Detector are in the following sections. 


\subsubsection{Near and Far Detector Fiducial Volume Crite- ria}

Near Detector has a cylindrical fiducial volume, having radius of 0.8 $\mathrm{m}$ and $3 \mathrm{~m}$ in length, such that

$$
\begin{gathered}
\sqrt{\left(X_{\mathrm{vtx}}-X_{0}\right)^{2}+\left(Y_{\mathrm{vtx}}-Y_{0}\right)^{2}}<0.8 \mathrm{~m}, \\
0.81009 \mathrm{~m}<Z_{\mathrm{vtx}}-0.0392 \mathrm{~m}<4.07710 \mathrm{~m},
\end{gathered}
$$

where $X_{0}=1.4828 \mathrm{~m}$ and $Y_{0}=0.2384 \mathrm{~m}$ are the Near Detector coordinates of the coil hole centre at the front face. All the events which are recorded with a track vertices out of the calorimeter containment, i.e, the plane before 14 and beyond 68 plane are removed. Total fiducial volume mass of the Near Detector is 23.7 tons.

The Far Detector also has a cylindrical fiducial volume, with a radius of $3.7 \mathrm{~m}$ from the centre of the detector and $50 \mathrm{~cm}$ away from the front and back planes of the two super modules. The total fiducial mass of the Far Detector is 4.17 kilotons (out of 5.4 kilotons in total).

\subsection{Event Selection Performance}

After all the preselection procedure, the standard selector 'RHC0350Std' selector, which contains a serious of selection cuts in order along with a charge sign cut which selects all the positive muons. This selector selects only the $\bar{v}_{\mu}$ CC events. The performance of the standard RHC selector is shown in Fig 6.5 .

Table 6.1: The wrong sign background and NC contamination

\begin{tabular}{ccc}
\hline \hline Detector & Wrong Sign Background & NC Contamination \\
\hline Near & $4.2 \%$ & $1.95 \%$ \\
Far & $4.4 \%$ & $1.25 \%$ \\
\hline
\end{tabular}

\subsection{Previous MINOS Analysis}

MINOS analysis generally use the Near Detector neutrino energy spectrum to predict the Far Detector spectrum in the case where there are no neutrino oscillations. For the sterile analysis at higher $\Delta m_{41}^{2}$ oscillation 

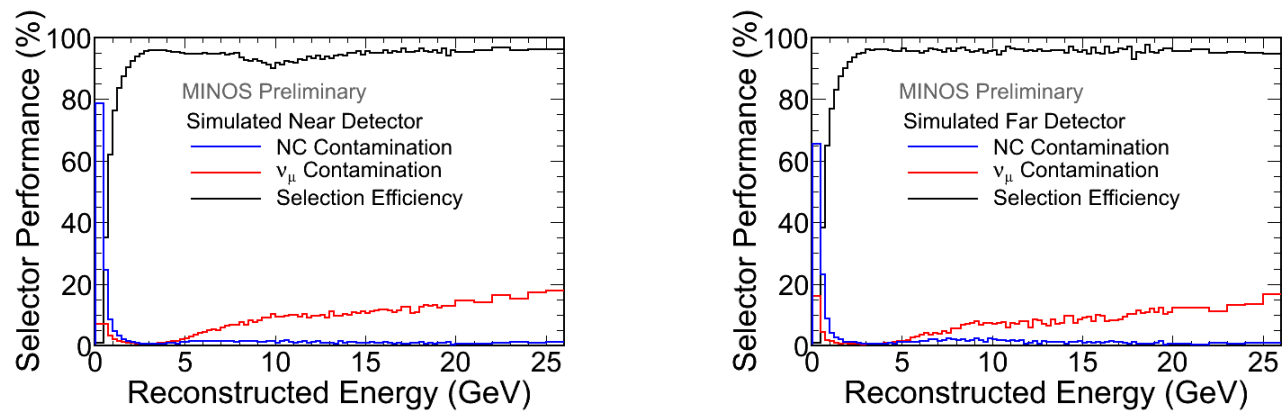

Figure 6.5: Performance of the current RHC selection (the $\mathrm{CC}$ and NC separation variable $($ roI $D)>0.3$ and product of momentum and charge $(q p)>0)$ in the Near and Far Detector, which is essentially the CC selection. The dashed lines shows the contamination before the selection and the solid shows efficiency and contamination after the selection. The $v_{\mu}$ contamination rises at higher energies as it is difficult to assign charge for these tracks.

could happen at Near Detector, in that case we cannot use the Near Detector spectrum to predict the Far Detector spectrum. In previous sterile analyses we predict the Far Detector spectrum via a Far over Near ratio method and fit the predicted spectrum with data. The Far Detector prediction is given by,

$$
F D_{\text {Pred }}=\frac{F D_{\text {OscMC }}}{N D_{\text {OscMC }}} \times N D_{\text {data }} .
$$

We take ratio of the oscillated FD MC spectrum with the oscillated ND MC spectrum and multiply the ratio by the ND data spectrum to the Far Detector prediction. And a $\chi^{2}$ minimization is performed to calculate how well the prediction agree with data. All the systematics are added as a nuisance parameter. The Poisson $\chi^{2}$ is given by,

$$
\chi^{2}=2 \sum_{i=1}^{N}\left[e_{i}-o_{i}+o_{i} \ln \frac{o_{i}}{e_{i}}\right]+\sum_{j=1}^{N} \frac{\epsilon_{j}^{2}}{\sigma_{j}^{2}}
$$

\subsection{New Analysis Strategy}

Since we are looking for the perturbations from the standard three flavor oscillation, systematics have to be fully accounted for. In this analysis 
the systematics are also allowed to vary in the fitting, for this reason we incorporate the systematics into a covariance matrix, where in previous analyses this is included as a nuisance parameter in the fit [106]. We define the $\chi^{2}$ as,

$$
\chi^{2}=V^{T} C^{-1} V+\frac{\left(N_{D}-N_{M C}\right)^{2}}{\sigma_{M C}^{2}}+\frac{\left(\left|\Delta m_{32}^{2}\right|-0.0025\right)^{2}}{0.0005^{2}}
$$

where $V$ is defined as $\left(D_{i}-M C_{i}\right)$ and $C^{-1}$ is defined as the inverse of the covariance matrix, which includes the sum of the statistical and the systematics uncertainty. The $\sigma_{M C}^{2}$ accounts for the uncertainty on the overall rate measured at the Near Detector and it is taken as $50 \%$. The last term represent a penalty term, which is a small constraint on $\Delta m_{32}^{2}$ in order to prevent it from varying to unrealistic values and becoming degenerate with the sterile mass splitting over the whole phase-space. The total covariance matrix is defined as,

$$
C=\left(\begin{array}{cccc}
\delta\left(\frac{f}{n}\right)_{1} & \cdots & \cdots & 0 \\
0 & \delta\left(\frac{f}{n}\right)_{2} & \cdots & 0 \\
\vdots & \vdots & \ddots & \vdots \\
0 & 0 & \cdots & \delta\left(\frac{f}{n}\right)_{N}
\end{array}\right)+\sum_{i=1}^{N} M_{i}
$$

where the first term represents the statistical covariance matrix and second term is the sum of all systematics matrices. The elements of the statistical matrices are the statistical uncertainty on the predicted $F / N$ ratio. Here the errors are obtained by propagating the ND and FD event rate uncertainty using standard error propagator.

The oscillation parameters used to make the three flavor fake data are given in Table 6.2

Table 6.2: Three flavor oscillation parameters

\begin{tabular}{cc}
\hline \hline Parameters & Three flavor value \\
\hline$\Delta m_{23}^{2}$ & $2.46 \times 10^{-3} \mathrm{eV}^{2}$ \\
$\Delta m_{21}^{2}$ & $7.59 \times 10^{-5} \mathrm{eV}^{2}$ \\
$\sin ^{2} \theta_{23}$ & 0.41 \\
$\sin ^{2}\left(2 \theta_{13}\right)$ & 0.09 \\
$\sin ^{2} \theta_{12}$ & 0.318 \\
\hline
\end{tabular}




\subsection{Binning Scheme}

The $\chi^{2}$ distribution assumes a Gaussian probability. To ensure the Gaussian probability assumption it is required to have a reasonable number of expected events per bin, otherwise the $\chi^{2}$ minimization values differ significantly from the Poisson probability. The binning scheme of the analysis is chosen such that we will expect minimum of 15 events per bin at the Far Detector, at this rate the $\chi^{2}$ minimization between Poisson and Gaussian differ in the order of $10^{-3}$. The Fig 6.6 shows the percent difference between Poisson and Gaussian distribution.

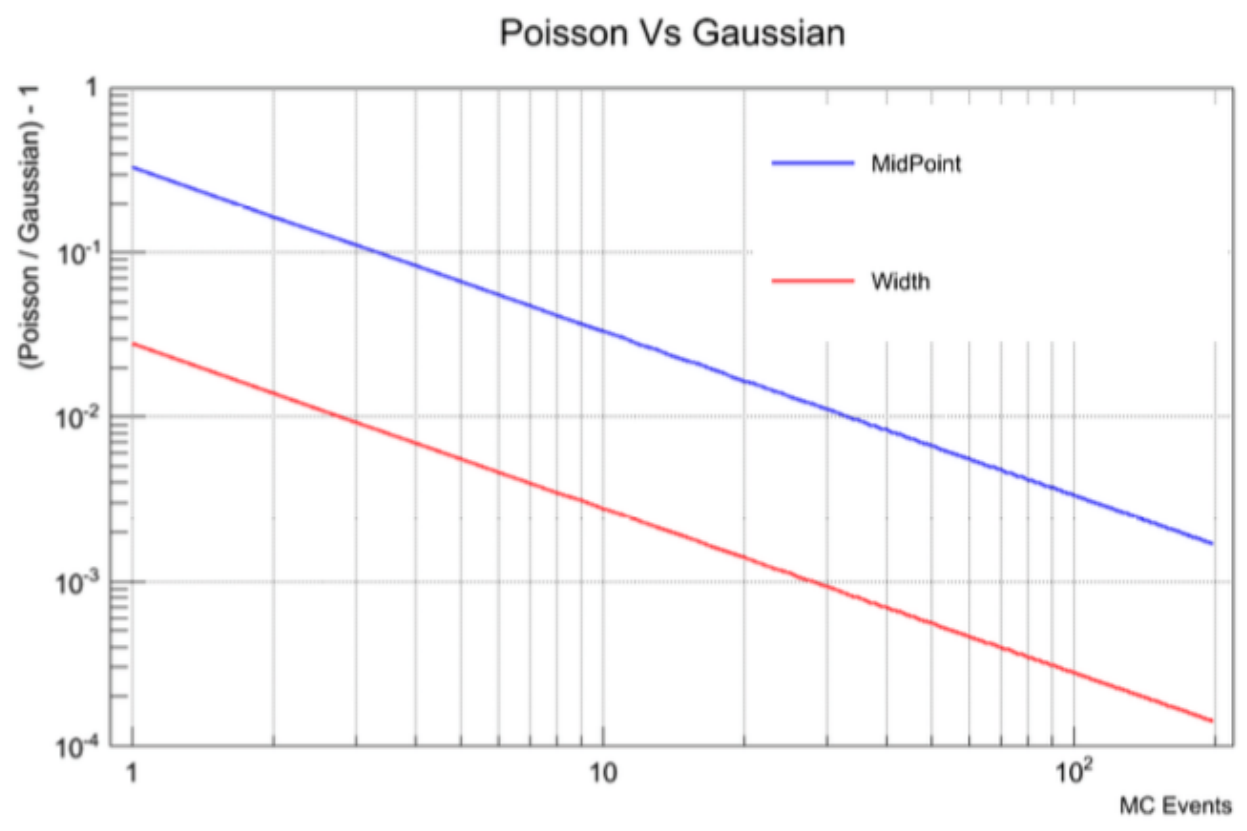

Figure 6.6: This plot shows the percent difference between Poisson and Gaussian distribution as a function of the number of expected events in some arbitrary bin. 

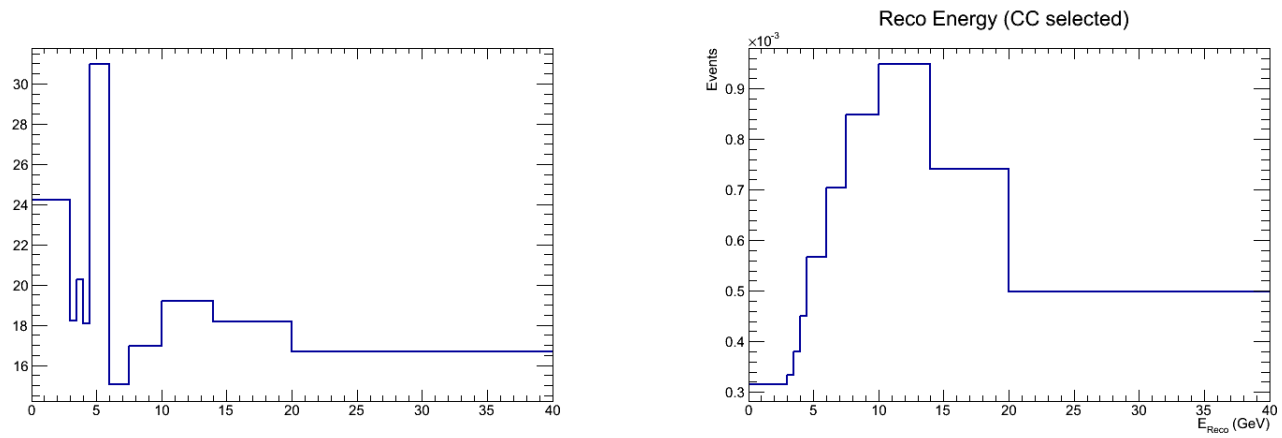

Figure 6.7: Shows the re-binned spectrum for the Far Detector expected number of events (left) and Far-over-Near ratio (right).

\subsection{Statistical Uncertainty}

Statistical uncertainty in each bin is calculated from the statistical covariance matrix created using the $F / N$ ratio. It is equal to the diagonal elements in the statistical error matrix. Statistical uncertainty in the Far and Near detectors are taken as $1 / \sqrt{N}$, then this errors are propagated to get the error on $F / N$ ratio. The statistical matrices are diagonal matrices due to the statistical error in bin $i$ being uncorrelated to that of bin $j$ and $100 \%$ correlated with itself.

$$
\begin{gathered}
R_{i}=F_{i} / N_{i} \\
\sigma_{i}^{2}=\left(\frac{\partial R}{\partial F}\right)^{2} \cdot(\delta F)^{2}+\left(\frac{\partial R}{\partial N}\right)^{2} \cdot(\delta N)^{2}
\end{gathered}
$$

substituting $(6.9)$ in $(6.10)$, we get,

$$
\begin{gathered}
\sigma_{i}^{2}=\left(\frac{1}{N_{i}}\right)^{2}\left(\sqrt{F_{i}}\right)^{2}+\left(\frac{-F_{i}}{N_{i}^{2}}\right)^{2}\left(\sqrt{N_{i}}\right)^{2} \\
\sigma_{i}^{2}=\frac{F_{i}}{N_{i}^{2}}+\frac{F_{i}^{2}}{N_{i}^{3}} .
\end{gathered}
$$

Assuming a high rate in ND, the error can be approximated as,

$$
\sigma_{i}^{2}=R_{i} / N_{i}
$$


where $N_{i}$ and $F_{i}$ are predicted number of events in the Near and Far Detector respectively.
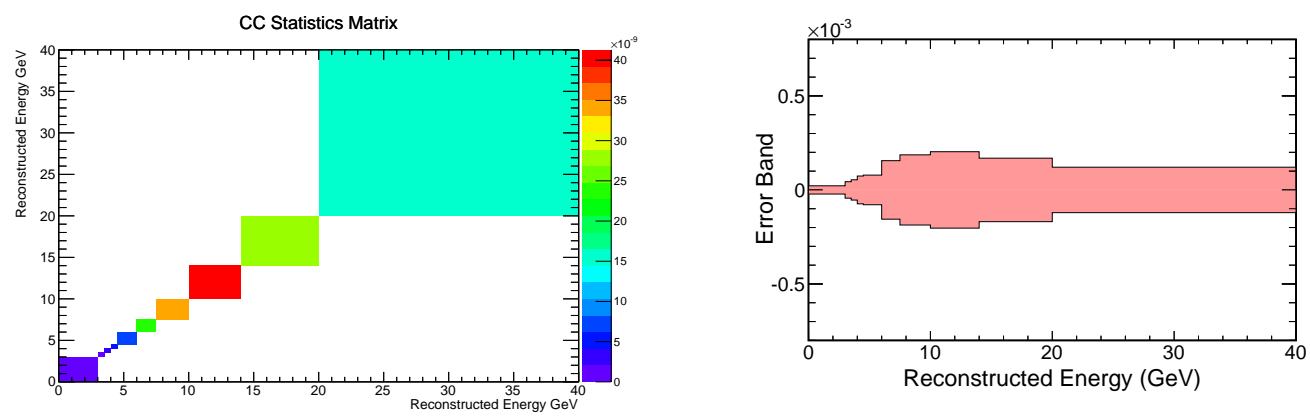

Figure 6.8: Statistical Covariance matrix (left) and corresponding Error band on $F / N$ ratio (right), generated from the covariance matrix.

\subsection{Systematics Uncertainties}

Various systematic uncertainties have been re-evaluated for this analysis and these are discussed in the following section.

\subsubsection{Hadron Production}

To obtain the uncertainty due to hadron production, we fit the MC differential cross-section for $\pi$ and $K$ production in $\mathrm{pC}$ collisions with a parametrization based on BMPT. This results in the best fit parameter values, errors and correlation matrix [108]. The fit is then compared to data from NA49 and the parameter errors are increased to cover the data, while preserving the correlations. This would introduce a large uncertainty on a single detector experiment but has $<3 \%$ effect on $F / N$ ratio [109]. 

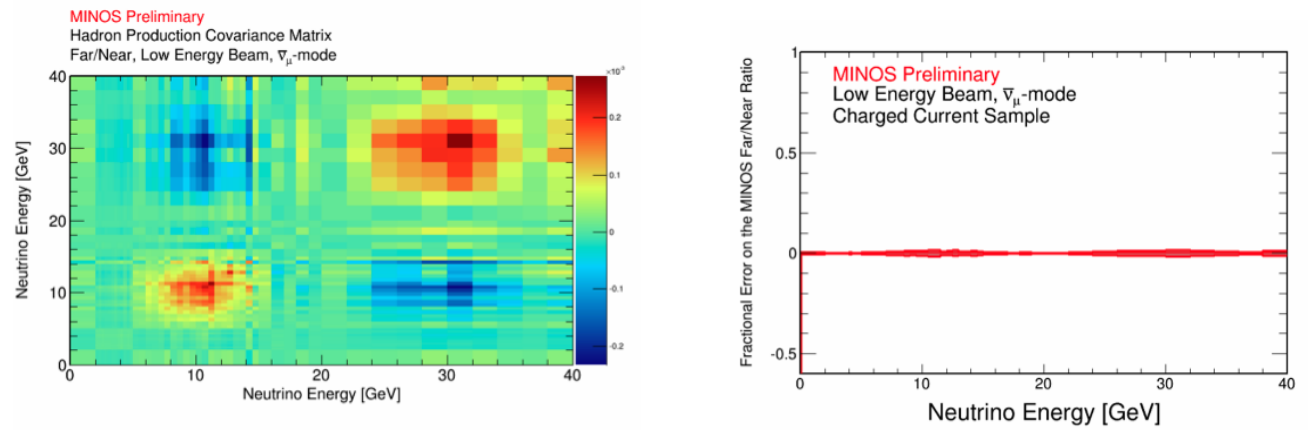

Figure 6.9: The error on hadron production on $F / N$ ratio (left). And the hadron production covariance matrix (right).

\subsubsection{Normalisation Systematics}

A $1.6 \%$ uncertainty in the relative reconstruction efficiency of the Near and Far Detector is determined by a scanning study.
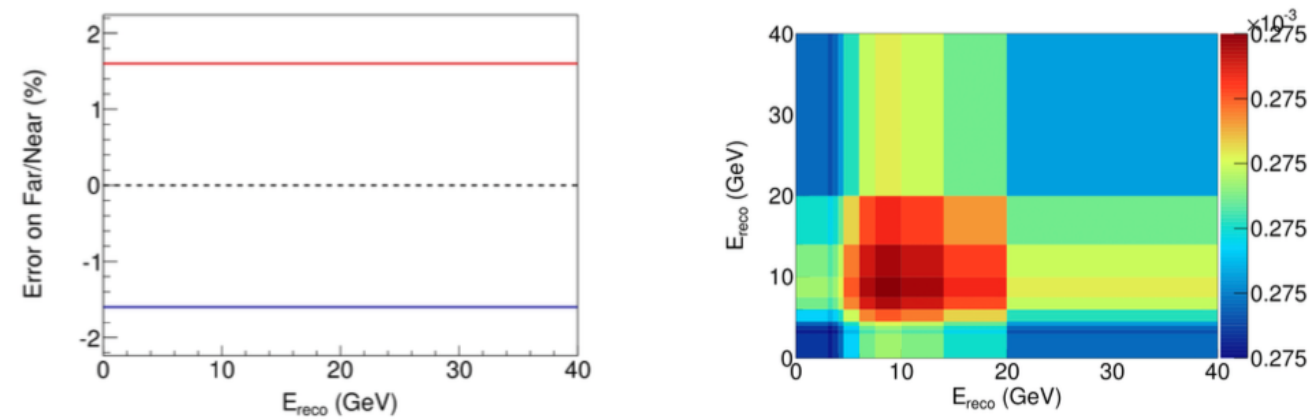

Figure 6.10: The error on $\mathrm{CC}$ normalisation on $F / N$ ratio (left). And the covariance matrix (right).

\subsubsection{Acceptance Systematics}

The uncertainty on the energy depends upon the acceptance and efficiency of the ND for CC and NC events. The uncertainty is evaluated by varying event selection requirements in data and $\mathrm{MC}$ to probe known weaknesses in the simulation [110]. Any shift in the data-MC agreement 
as the requirements were varied and taken as the systematic uncertainty. The systematics are energy dependent and include correlations between different bins. The fiducial volume criteria were varied both parallel and perpendicular to the focusing plane and along the detector axis. Also varied the treatment of tracks entering the magnetic coil, the track containment criteria near the side edges, downstream end of the detector and the region in which the scintillator sampling changes. The shower energy scale and tack energy scale is also varied by $+1 \sigma$ and $-1 \sigma$. The acceptance at Near Detector is defined as,

$$
\frac{(\text { data } / \mathrm{MC})_{\text {shifted }}}{(\text { data } / \mathrm{MC})_{\text {nominal }}}-1
$$

where the nominal ratio is the ratio with standard selection cut and the shifted ratio is the ratio of modified selection cut. This double ratio is a measure of mis-modeling in the simulation [111. The remaining systematics such as track energy, shower energy, cross section etc, are encoded in NuSystematics as $\pm 1 \sigma$, which is used in all previous MINOS analysis. These systematics, the error bands are construct from the nominal and shifted far over near ratio and is defined by,

$$
\frac{(\text { far } / \text { near })_{\text {shifted }}}{(\text { far } / \text { near })_{\text {nominal }}}-1
$$
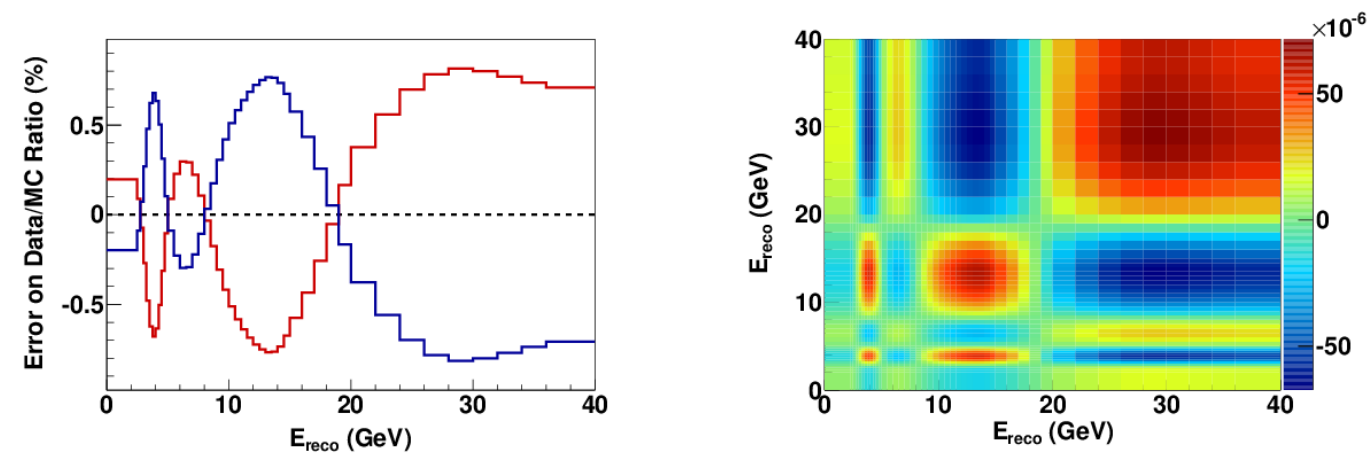

Figure 6.11: The error band made for fiducial volume tightening, here the shifted distribution is constructed by tightening the fiducial radius from $80 \mathrm{~cm}$ to $60 \mathrm{~cm}$, and the ratio with the nominal is taken (left). The corresponding covariance matrix (right). 

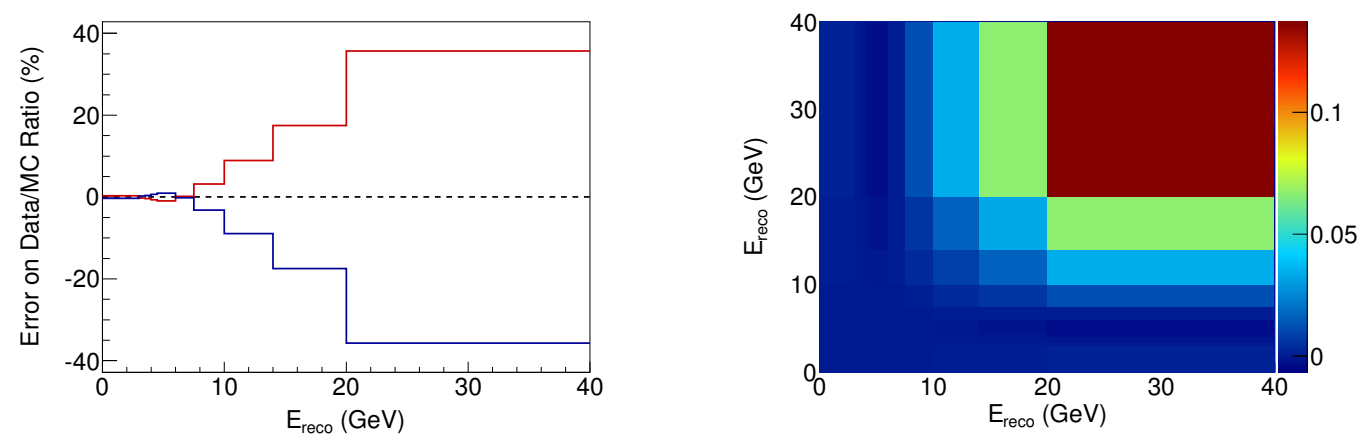

Figure 6.12: Shows the mis-modeling of back exiting tracks. The shifted distribution is constructed by removing all events with a track ending with in 10 planes of the end of the Near Detector (left). And the corresponding covariance matrix (right).
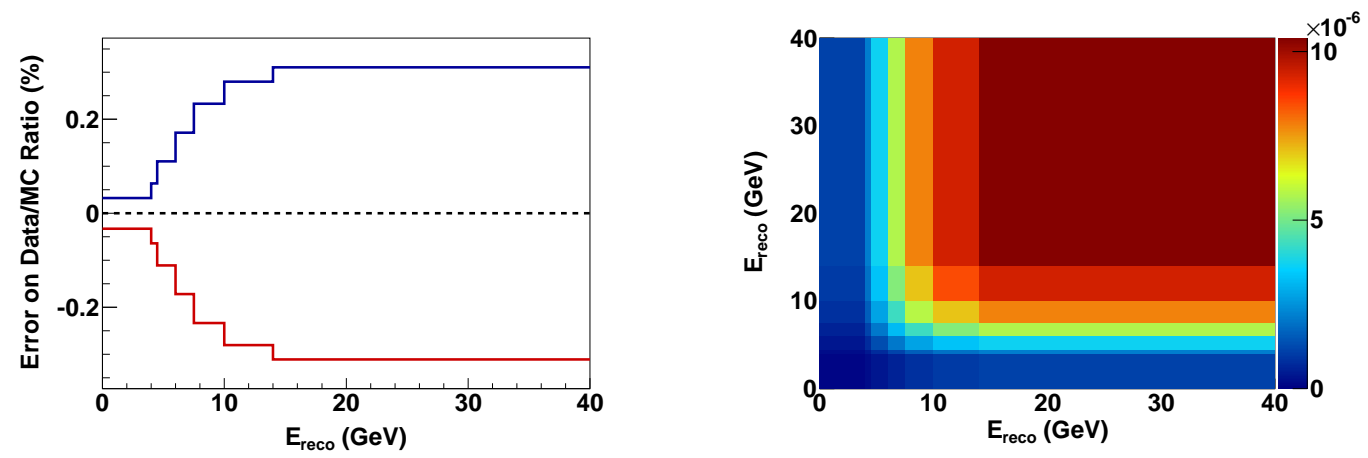

Figure 6.13: This shows mis-modeling of the spectrometer join region. The shifted distribution is constructed by removing all events with a track ending within 10 planes of the start of the spectrometer (left). And the corresponding covariance matrix (right). 

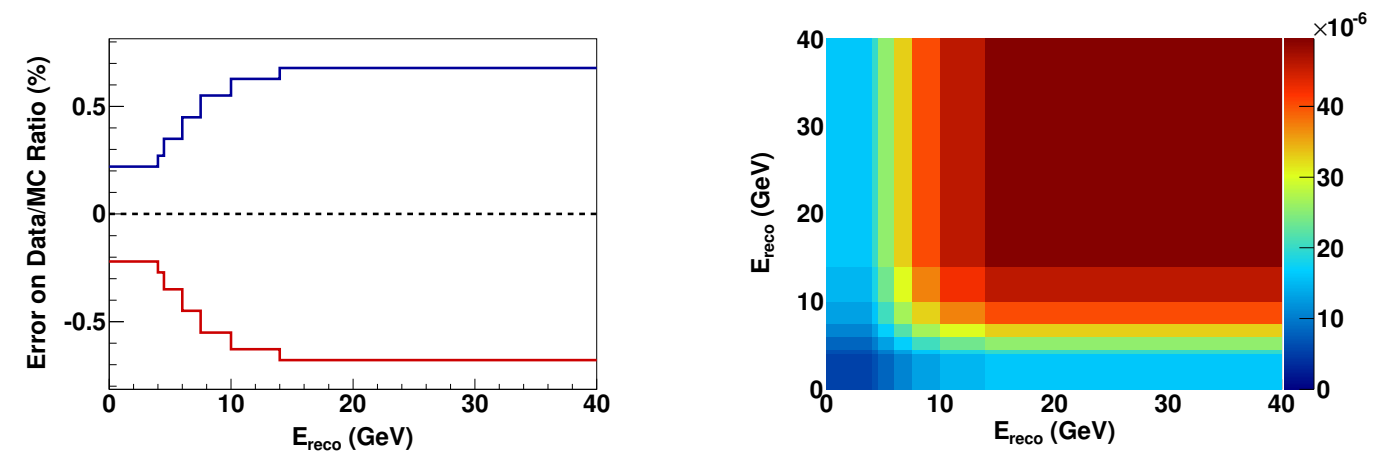

Figure 6.14: The mis-modeling of exiting tracks. The shifted distribution is constructed by turning off the containment cut (left). And the corresponding covariance matrix (right).

\subsubsection{Beam Optics systematics}

The uncertainty on the beam focusing is due is to the uncertainties in the position of beam line elements, proton beam-halo interactions at upstream of the target and the model of the magnetic horns used to focus pions. This introduces an uncertainty of $<2 \%$ in $4-10 \mathrm{GeV}$ in the $\mathrm{CC}$ sample with negligible effect at other energies and on the neutral current samples [112]. The main source of beam optic systematics are a) Horn current distribution - MC assumes that the skin depth is infinite but in reality it is not the case. b) Horn current mis-calibration-uncertainty on how accurately the horn current is measured. c) Horn one offset - this is the uncertainty on the displacement of the horn along the beam axis. 


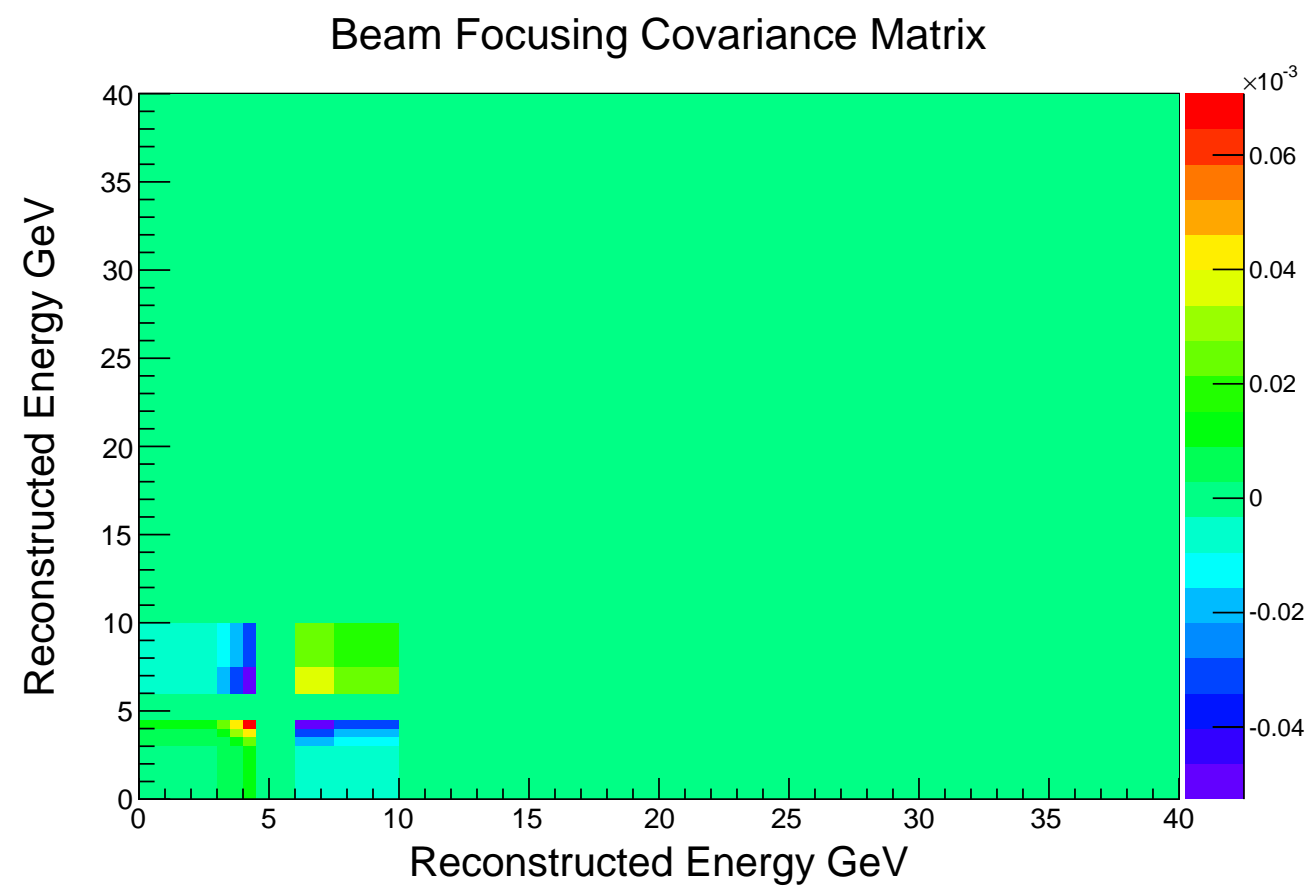

Figure 6.15: The beam optics systematics due to the horn current distribution on the $F / N$ ratio.

\subsubsection{Cross Section Systematics}

Cross Section uncertainties includes the systematics from total CC cross sections as well as cross section from quasi elastic scattering, baryon resonance production and deep inelastic scattering. This would be significant uncertainty in a one detector experiment but in MINOS it has $<1 \%$ effect on $F / N$ CC samples [113]. 

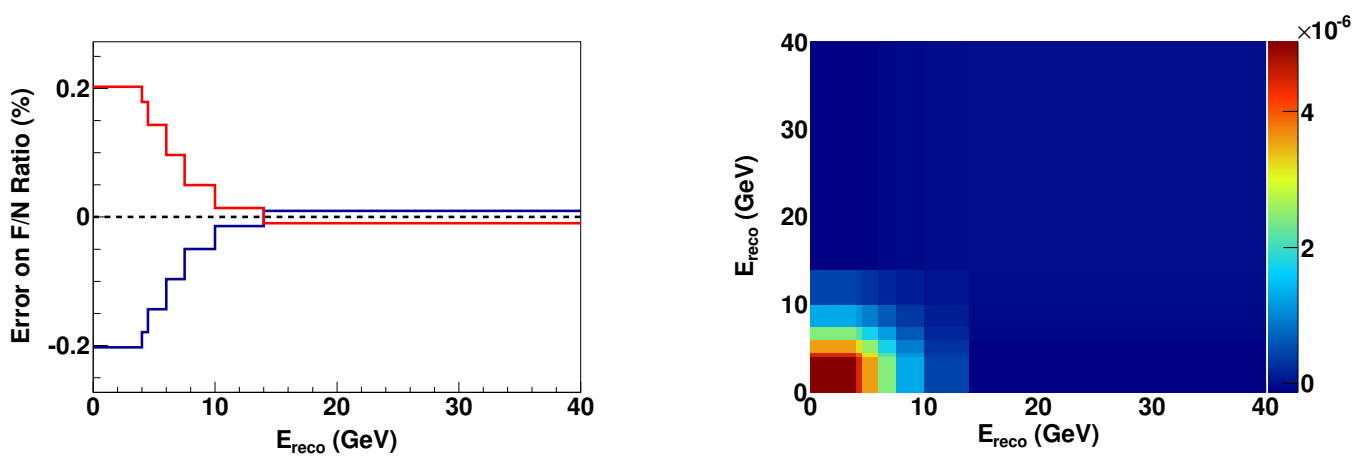

Figure 6.16: Cross section error band for the value of the axial vector mass $\left(M_{A}\right)$ for quasi-elastics events obtained by varying it by 1.15 and 0.85 (left). And the corresponding covariance matrix (right).

\subsubsection{The Statistical and Systematic Error Band on $F / N$}

The MINOS RHC sample is statistically limited, hence it is not possible to observe large variations when we add the systematic uncertainties. The Fig 6.17 shows effect of these uncertainty on $F / N$ ratio.
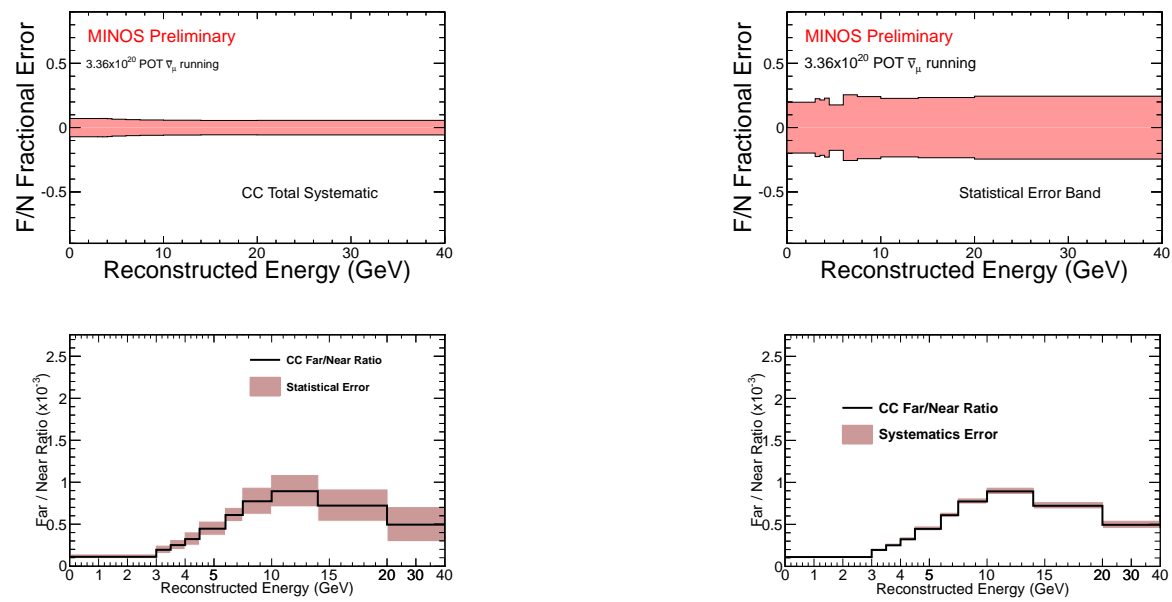

Figure 6.17: Statistical and systematics error band and for the $F / N$ ratio. The statistical error dominates due to low statistics in FD for the antineutrino CC samples. 


\subsection{Sensitivity}

The addition of the fourth state will introduces more mixing angle, mass splitting and phases. The additional parameters are $\theta_{24}, \theta_{14}, \theta_{34}$, $\Delta m_{41}^{2}$ and the three $\mathrm{CP}$ violating phases, $\delta_{14}, \delta_{24}$ and $\delta_{13}$. CP violating phases are not sensitive to MINOS especially in the charged current samples, so they are fixed to zero in the fitting, $\theta_{14}$ is also not sensitive to MINOS so its value also set to zero in the fit. During the fit, the parameters $\Delta m_{23}^{2}, \theta_{23}, \theta_{34}$ are allowed to free for different values of $\theta_{24}$ and $\Delta m_{41}^{2}$. By minimizing the $\chi^{2}$ from equation 6.7, the sensitivity for the sterile neutrino has been calculated. We also profile the mass hierarchy and octant of $\theta_{23}$ during the fit and pick up the best $\chi^{2}$ value from this four combination (two mass hierarchy and two octant). This sensitivity contour contains only statistical uncertainty on it, we exclude everything right to the curve at $90 \%$ C.L.

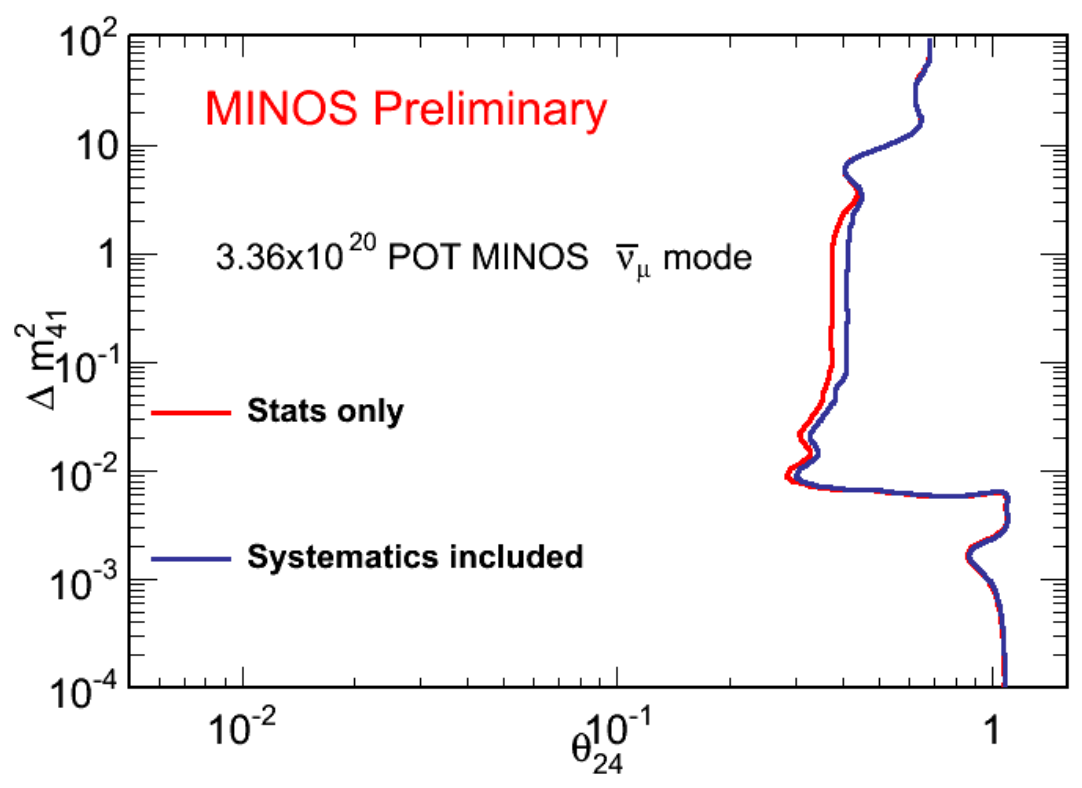

Figure 6.18: The sensitivity for sterile neutrino at $3.36 \times 10^{20}$ POT antineutrino optimized mode running. Only $\mathrm{CC}$ events with positive charged track $\left(\mu^{+}\right)$are used in the analysis. 


\subsection{Sensitivity with Systematics}

The sensitivity for the sterile searches has been evaluated by the addition of the total systematic uncertainty, the effect of individual systematics are studied and the dominant contribution comes from the acceptance systematics. All other systematics have very small effect on the sensitivity, this is because our sample is statistically limited. The systematics are added separately to observe the effect from the individual components.

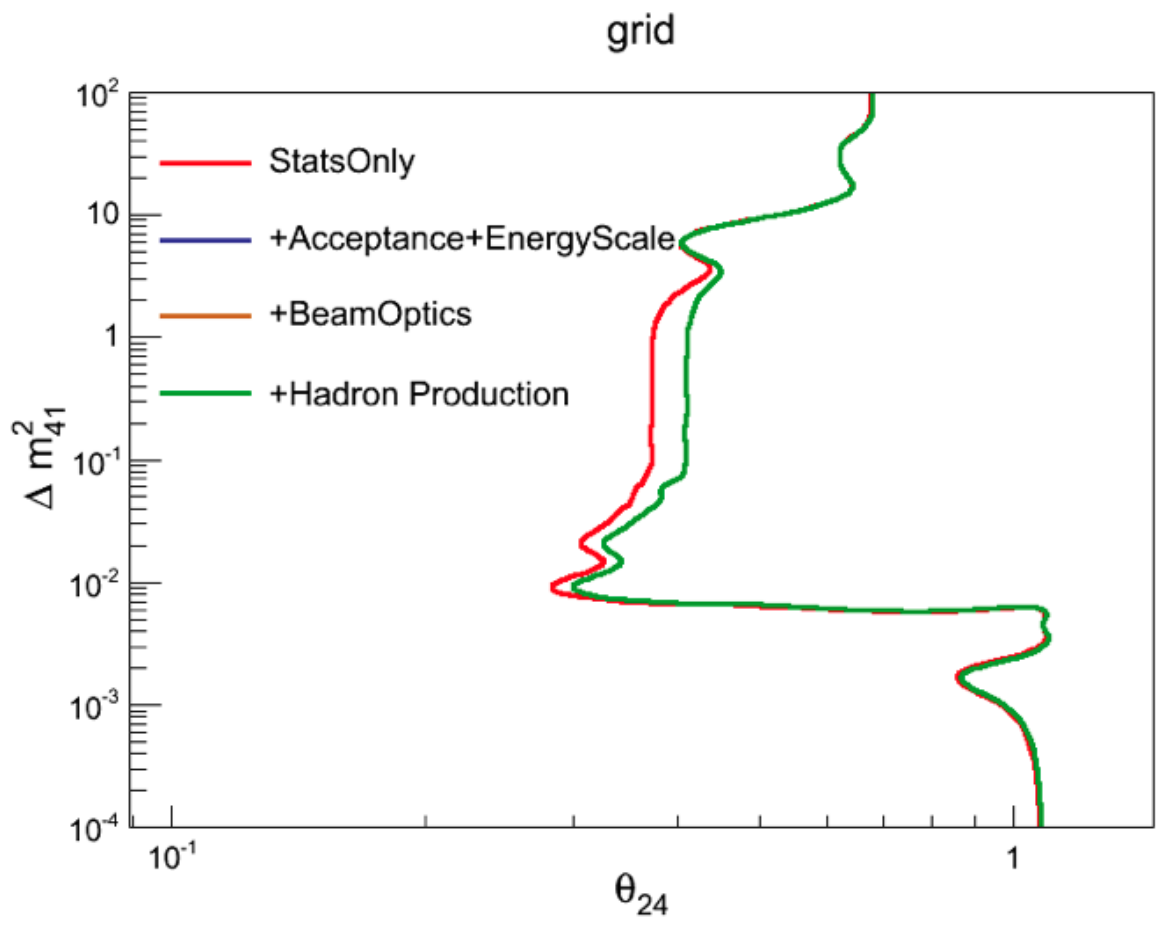

Figure 6.19: The sensitivity for sterile neutrino at $3.36 \times 10^{20}$ POT antineutrino optimised mode running. Only CC events with positive charged track $\left(\mu^{+}\right)$are used in the analysis. Figure shows the contribution of systematics effect on the sensitivity

The MINOS sensitivity has been compared with the results from other experiments, like MiniBooNE and CCFR. As the MINOS is a two detec- 
tor experiment we can do a normalization measurement at Far Detector rather than a shape only measurement as MiniBooNE has been performed. CCFR is a two detector experiment running at very short baseline, so essentially sensitive to much higher mass squared splitting.

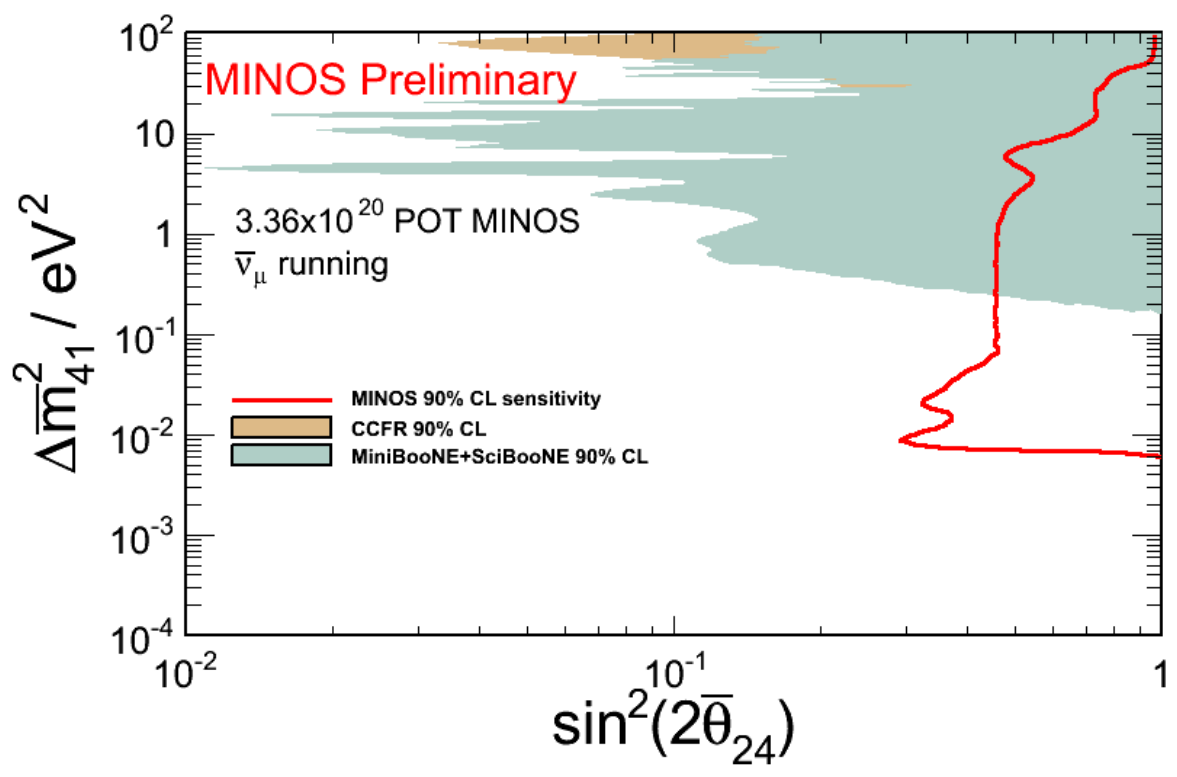

Figure 6.20: The sensitivity for sterile neutrino after including all systematics in the antineutrino optimised mode running, compared with the limit set by the other experiments.

\subsection{Extended Contour and Surface in $\Delta m_{41}^{2}$}

The $\chi^{2}$ surface is extended to the lower values of the $\Delta m_{41}^{2}$. The surface features are more visible if we make the $z$ values in log scale, which is shown in Fig 6.22. As $\Delta m_{41}^{2}$ goes to the lower values the problem due to degeneracy occurs, which is more clear in data contour. 

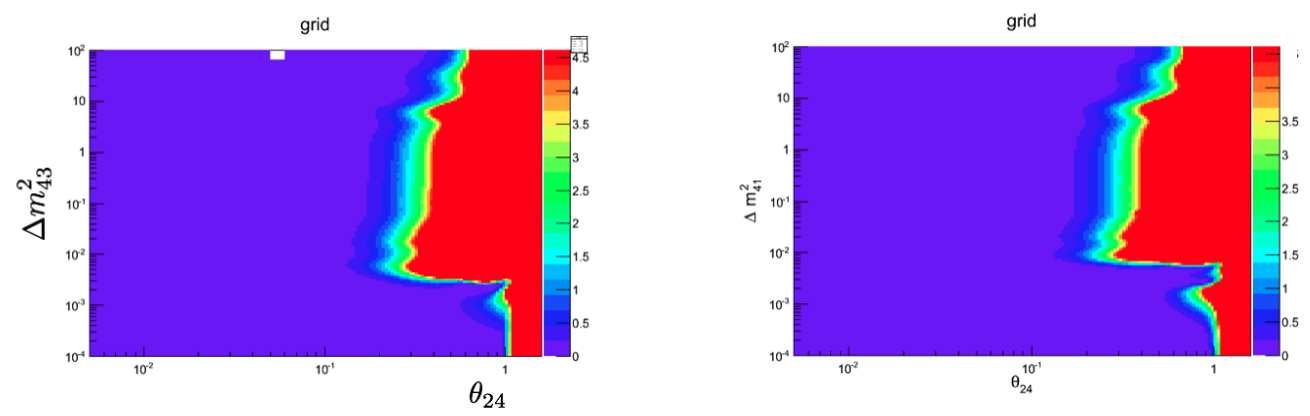

Figure 6.21: The left figure shows surface in terms of $\Delta m_{43}^{2}$ and right figure shows the surface in terms of $\Delta m_{41}^{2}$.

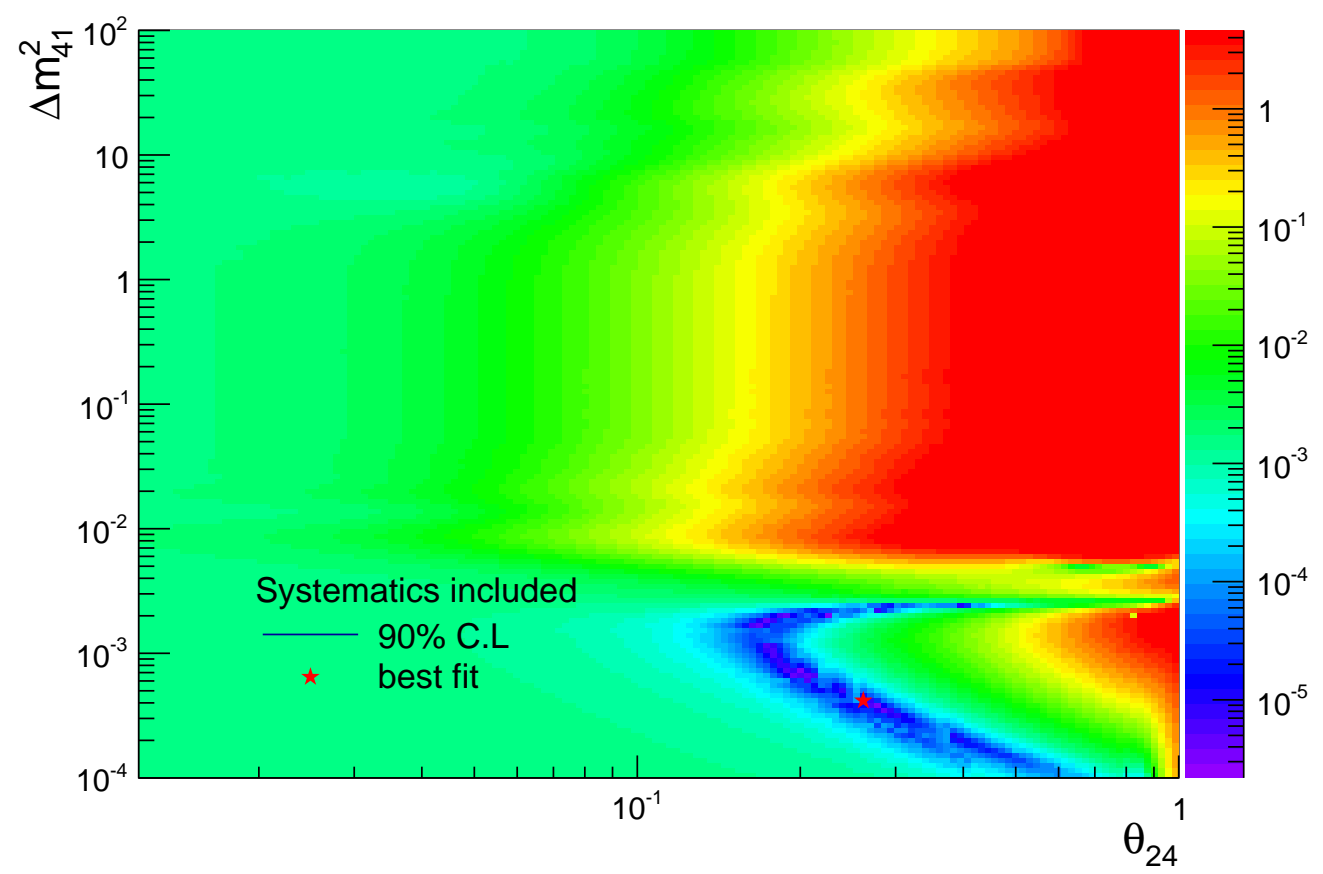

Figure 6.22: The surface is made in $\log z$ scale to see the the surface features at lower $\Delta m_{41}^{2}$ region. 


\subsection{Feldman Cousins}

Method of Feldman Cousins is used to find the correct coverage [114]. The value of $\Delta \chi^{2}$ per point, which corresponds to a specific confidence level is computed using this method. The basic principle of the FeldmanCousins method is to determine using Monte-Carlo simulation what $\Delta \chi^{2}$ gives the correct coverage ( $68 \%$ C.L, $90 \%$ C.L etc). And the $\Delta \chi^{2}$ distribution is created at each point in the parameter space using many fake experiments and the $\Delta \chi^{2}$ is calculated as,

$$
\Delta \chi^{2}=\chi_{\text {profile }}^{2}-\chi_{\text {best }}^{2}
$$

where the $\chi_{\text {profile }}^{2}$ is the value of $\chi^{2}$ evaluated at the fixed point in the parameter space and letting the atmospheric mixing parameter to be free, where as $\chi_{\text {best }}^{2}$ is the $\chi^{2}$ at the best fit point, where all five parameters are free in the fit.

Fake experiments are generated with bin-to-bin statistical and systematic fluctuations, incorporated by sampling from the multi-dimensional Gaussian with covariance matrix V. This can be achieved by taking the Cholesky decomposition of the error matrix $V$ ( $V$ should be a symmetric positive definite matrix) and then use that matrix for getting the fluctuated far/near ratio in the following way.

$$
V=C C^{T}
$$

Where $C$ is the lower triangular matrix and $C^{T}$ is the conjugate transpose of $C$. Then a vector $X$, is created where each elements are drawn from a unit Gaussian distribution with mean 0 and variance 1 . Then the fluctuated $F / N$ ratio is defined as

$$
R_{\mathrm{fluc}}=R_{\mathrm{mc}}+C^{T} X
$$

Fig. 6.23 shows the distribution of $\Delta \chi_{\text {profile }}^{2}$ and $\Delta \chi_{\text {best }}^{2}$ obtained from 2500 fake experiments by choosing $\Delta m_{41}^{2}=0.5 \mathrm{eV}^{2}$ and $\theta_{24}=0.2$, for $90 \%$, the up value of the $\Delta \chi^{2}$ is at 5.22 in this case including all systematics. 

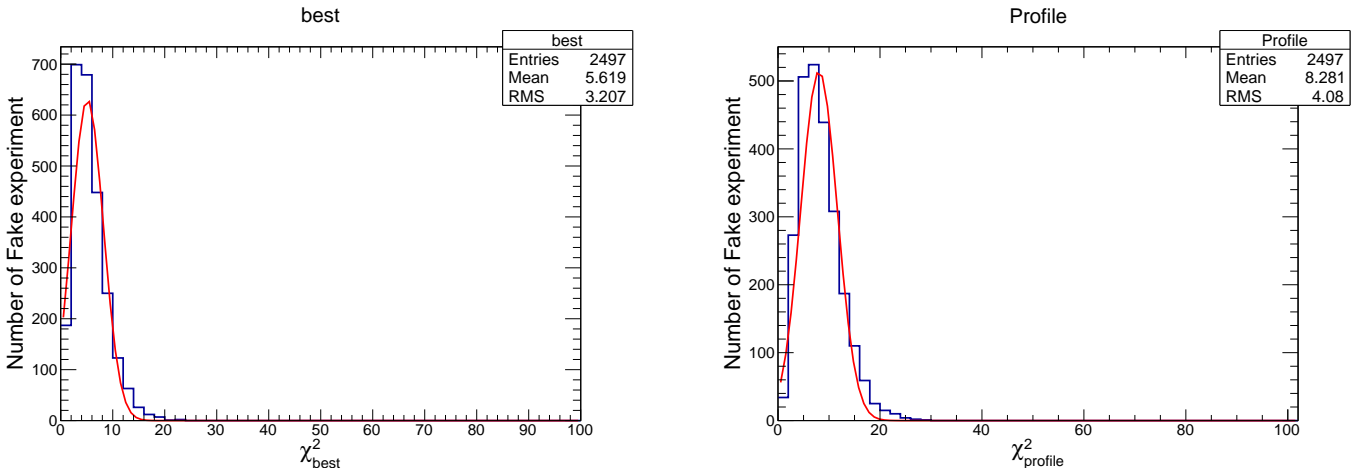

Figure 6.23: The $\Delta \chi_{\text {profile }}^{2}$ and $\Delta \chi_{\text {best }}^{2}$ distribution for 2500 fake experiment at $\Delta m_{41}^{2}=0.5 \mathrm{eV}^{2}$ and $\theta_{24}=0.2$, the $\Delta \chi_{\text {best }}^{2}$ is obtained by doing a 5 parameter fit where we allow both sterile and atmospheric parameters are allowed to be free.

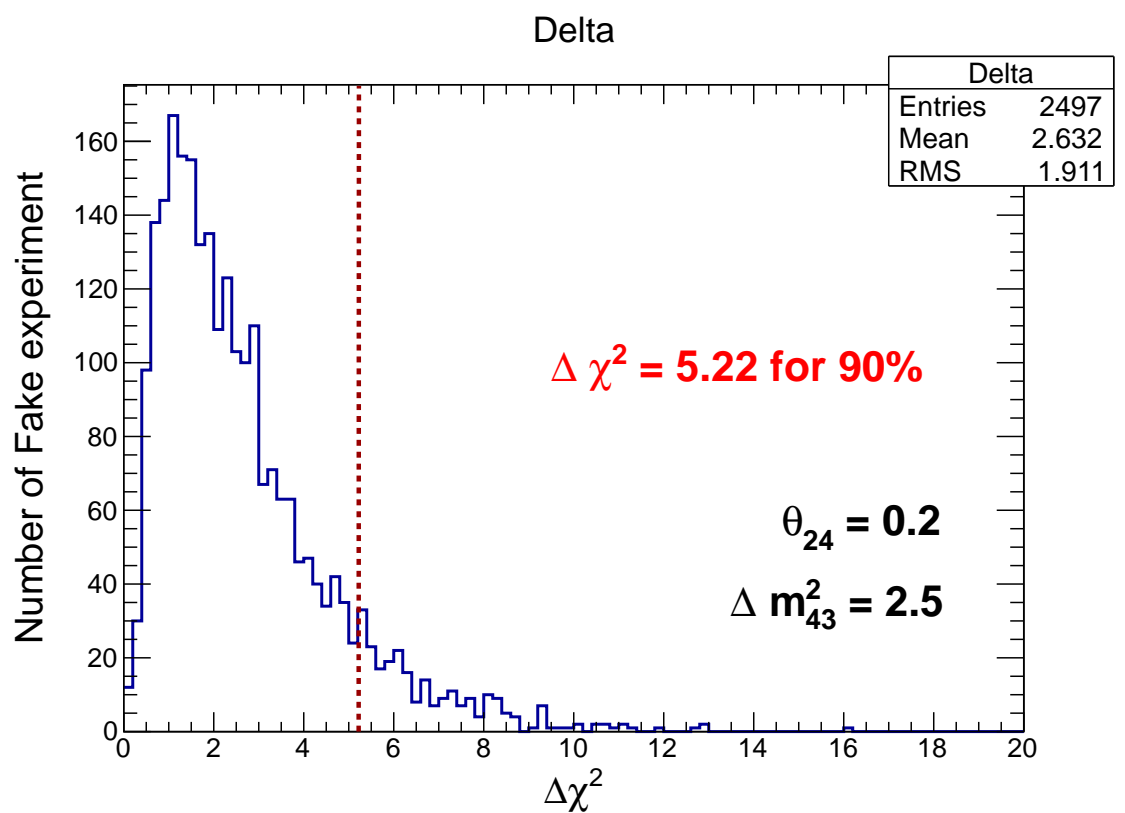

Figure 6.24: The $\Delta \chi^{2}$ distribution for 2500 fake experiment, here we are getting the up value for $90 \%$ C.L for 5.22, ideally it should be 4.61 for 90 $\%$ C.L for 2 degrees of freedom. 


\subsection{4 $F / N$ Ratio and Data Results}

In the Far Detector data we observed about $226 v_{\mu}$ CC events, the data is consistent with the three flavor prediction, the best fit for the sterile parameters are $\Delta m_{41}^{2}=6.6 \mathrm{eV}^{2}$ and $\theta_{24}=0.3$.

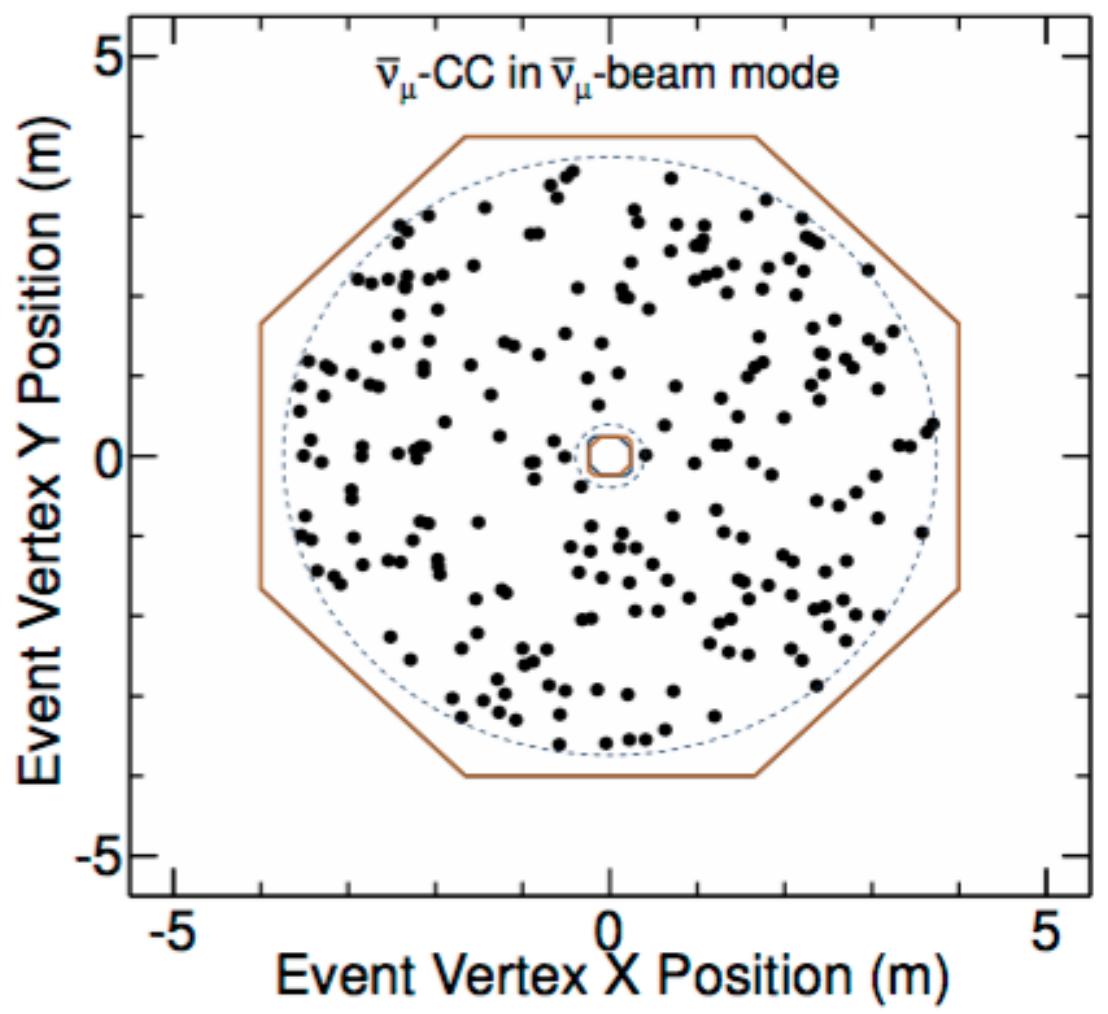

Figure 6.25: Distribution of track vertex for the $\bar{v}_{\mu}$ events in the MINOS Far Detector. A total of 226 events were observed after opening the data. 


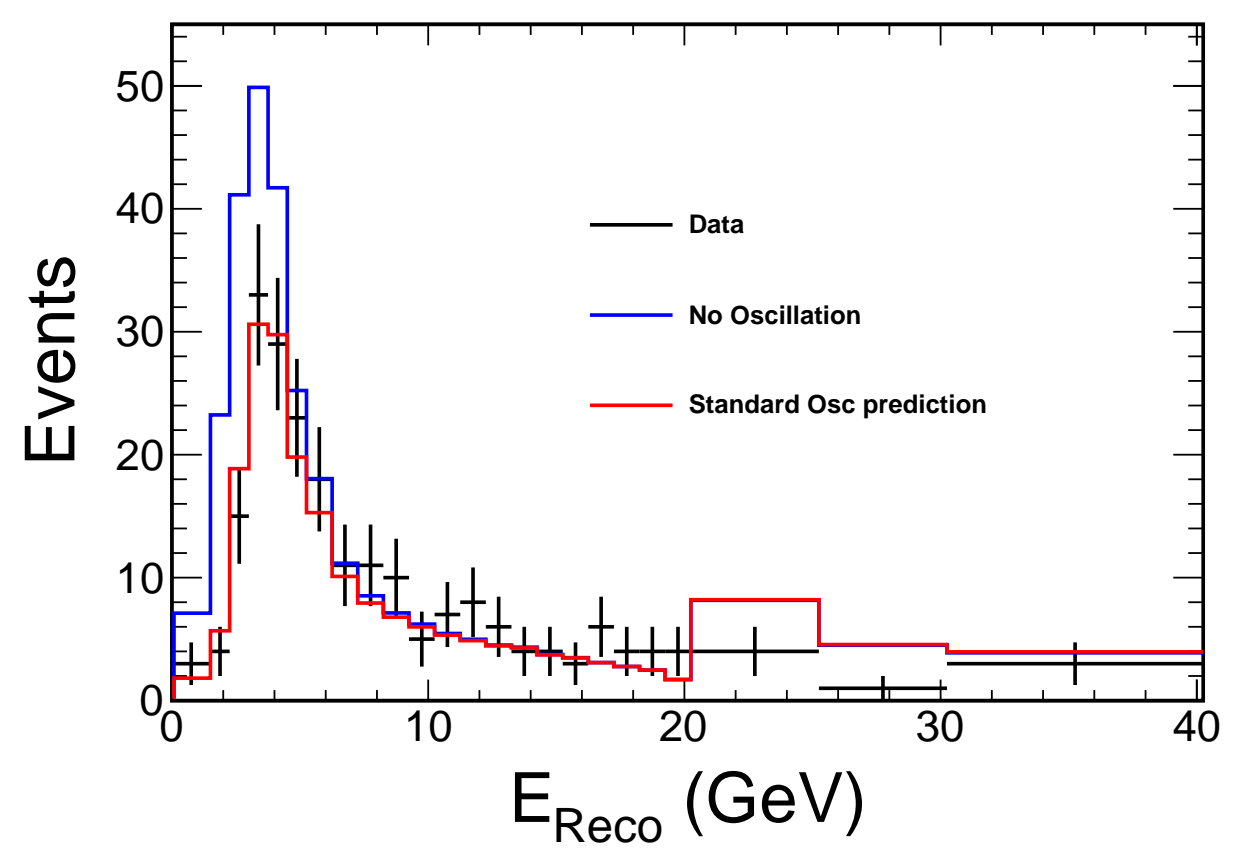

Figure 6.26: The far detector energy spectrum with three flavour prediction and with null oscillation are compared.
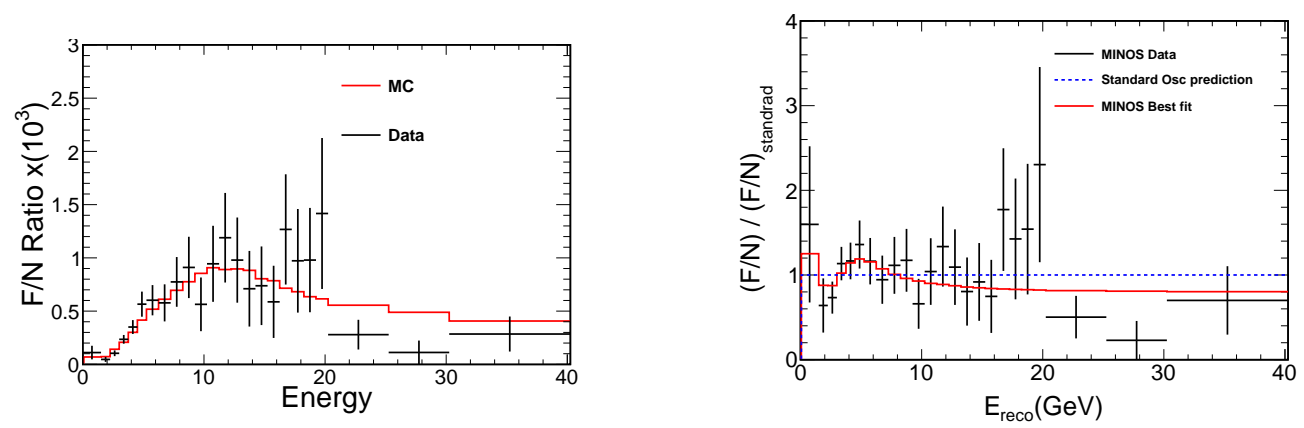

Figure 6.27: The $F / N$ ratio using data (left) and the double ratio(right) plots are shown, where the best fit values obtained from the fit are compared with data and three flavour prediction. 


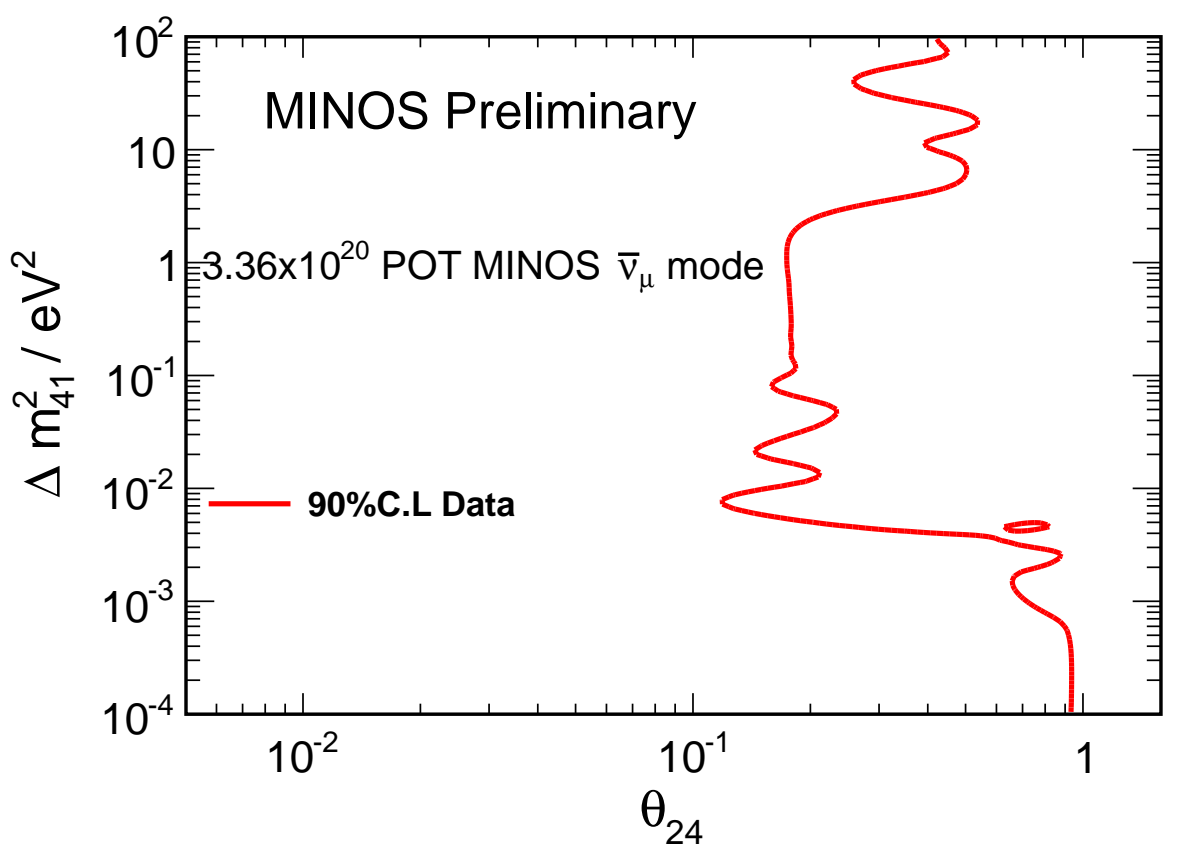

Figure 6.28: The $90 \%$ data limit obtained from antineutrino mode running. Everything right of the curve is excluded.

A limit for sterile neutrino is calculated using our $\bar{v}_{\mu}$ data sample, the limit span over 4 order of magnitude in $\Delta m_{41}^{2}$. For $\Delta m_{41}^{2}<10^{-2} \mathrm{eV}^{2}$, it is possible that one of the three mass squared splitting, $\Delta m_{41}^{2}, \Delta m_{42}^{2}$ or $\Delta m_{43}^{2}$ match the scale of oscillation of $\Delta m_{32}^{2}$ sector, which makes the solution degenerate with the standard three flavour oscillation solution, creating an island of allowed parameter space, which is visible in Fig 6.28.

\subsection{Feldman-Cousins Corrected Surface}

The FC correction is applied to the surface to generate the proper confidence limit free from any statistical fluctuations as described in Section 6.13 . 


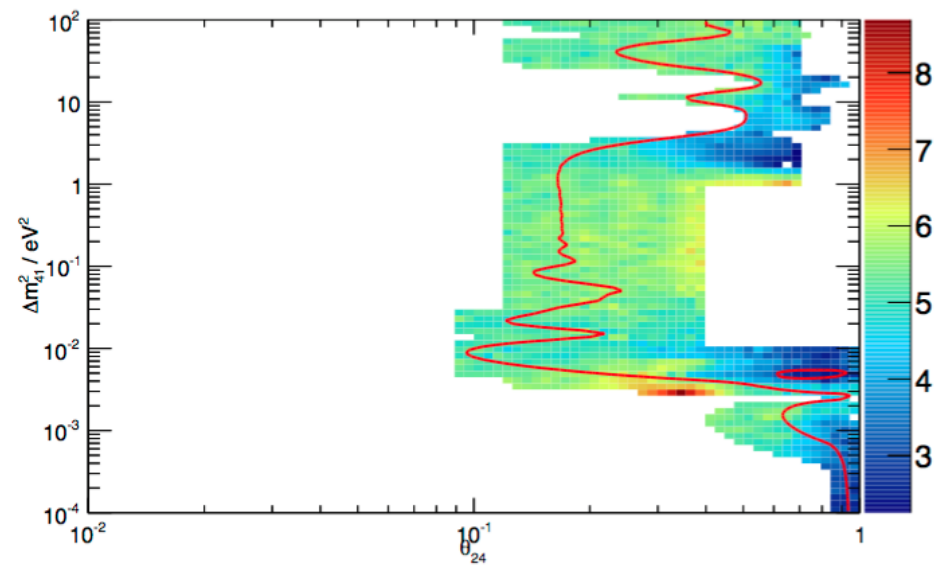

Figure 6.29: The $\Delta \chi^{2}$ surface generated for RHC data surface. The z-axis shows the value of $\Delta \chi^{2}$ at corresponding grid point.

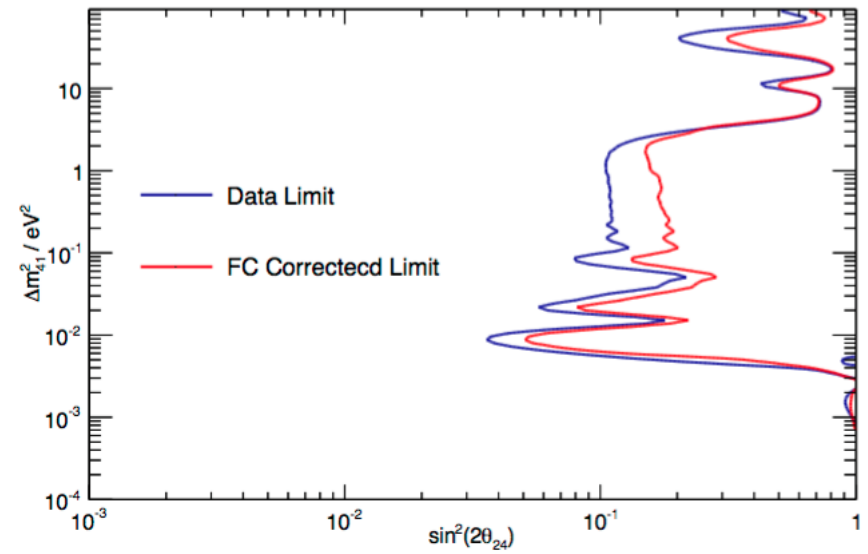

Figure 6.30: The raw limit and Feldman-Cousins limit. The surface is converted into $\sin ^{2} 2 \theta_{24}$. 


\subsection{Combination with Bugey}

In order to present the MINOS disappearance results on the same footing as appearance results from LSND and MiniBooNE, we will combine our disappearance limit $\left(\bar{v}_{\mu}\right)$ with the disappearance limit from Bugey reactor experiment $\left(\bar{v}_{e}\right)$. Since both limits originate from disappearance measurements, CP is independently conserved in both cases. Therefore, assuming CPT is conserved, the combined limit is applicable to appearance results from both neutrino and antineutrino running. It will be a more direct comparison in antineutrino mode. The appearance angle can be written in terms of matrix elements as

$$
\sin ^{2} 2 \theta_{\mu e}=4\left|U_{e 4}\right|^{2}\left|U_{\mu 4}\right|^{2} .
$$

These matrix elements are also measured at disappearance experiments. As described in [115] they can be written in terms of angles measured at Bugey and MINOS as,

$$
\begin{gathered}
\left|U_{e 4}\right|^{2}=\sin ^{2} \theta_{14}, \\
\left|U_{\mu 4}\right|^{2}=\sin ^{2} 2 \theta_{14} \cos ^{2} \theta_{14} .
\end{gathered}
$$

Therefore we can write the appearance angle in terms of the angles measured in both e and $\mu$ disappearance as,

$$
\sin ^{2} 2 \theta_{\mu e}=\sin ^{2} \theta_{14} \sin ^{2} \theta_{24}
$$

To construct the appearance angle limit from MINOS and Bugey, first we obtain the $\chi^{2}$ surface from the Bugey experiment as a function of $\Delta m_{41}^{2}$ and $\sin ^{2} 2 \theta_{14}$. This surface results from a GLoBES 2012 fit provided by P. Huber. It accounts for the new calculation of reactor fluxes. For each given $\Delta m_{41}^{2}$ we loop over each Bugey and MINOS point constructing the appearance angle using the above equation and calculate the total $\chi^{2}$. We determine the minimum $\chi^{2}$ for all $\Delta m_{41}^{2}$ and construct $\Delta \chi^{2}$ for all the points. We take the combined lower limit to be the allowed point for a given $\Delta m_{41}^{2}$ with the largest $\sin ^{2} 2 \theta_{\mu e}$. 


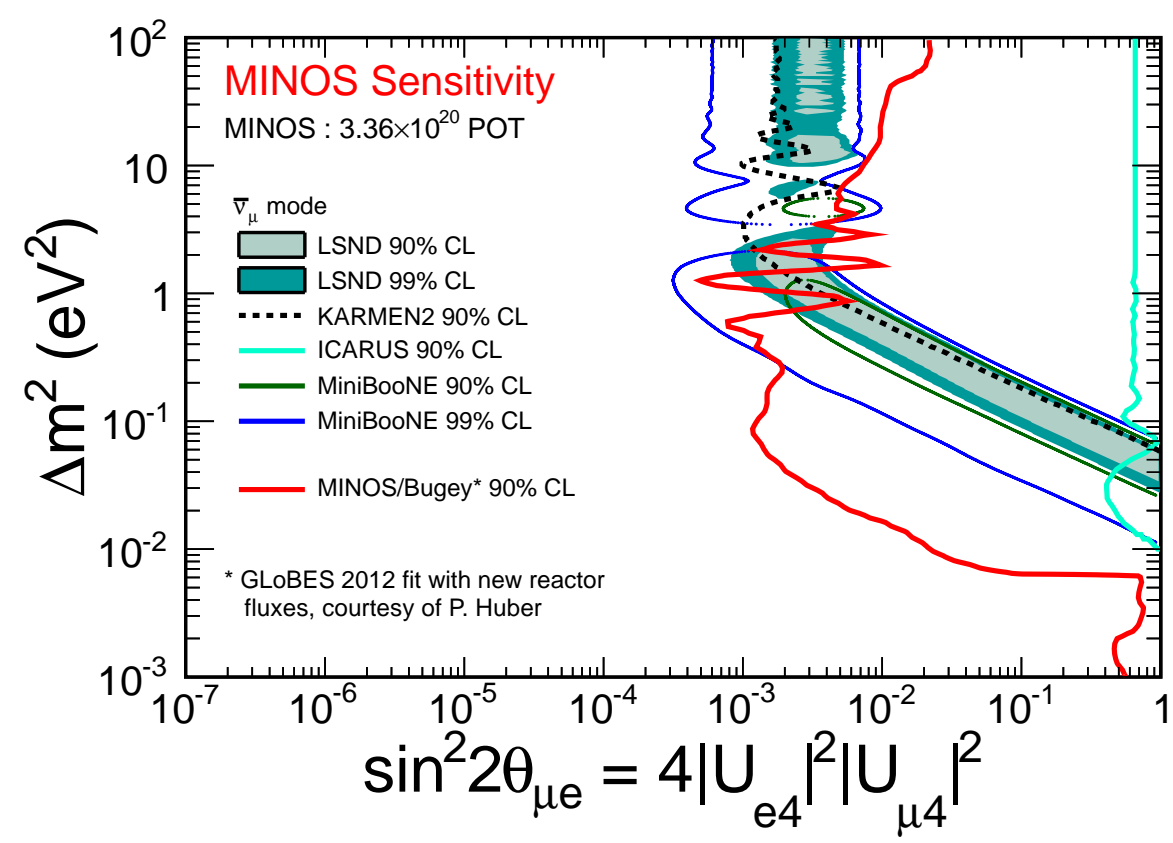

Figure 6.31: The Combined Bugey and MINOS limit shown in terms of appearance angle, which is same as that of probed by LSND and MiniBooNE.

\subsection{Future Antineutrinos in MINOS+}

MINOS+ running in NovA era, has a medium energy configuration, where neutrino energy peaks around $6 \mathrm{GeV}$, and 3 times more statistics than what we have in MINOS, because of this we can get much improved sensitivity in MINOS+, even for a year of MINOS+ running we will get a factor of two improvement in the sensitivity. 


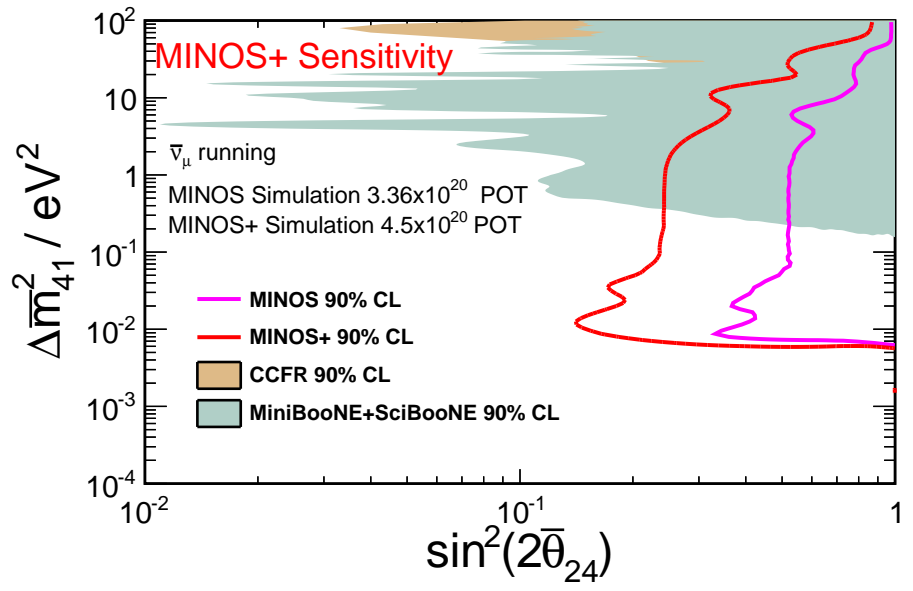

Figure 6.32: The combined MINOS and MINOS+ projected sensitivity for the antineutrino in MINOS+ era for a PoT of $4.5 \times 10^{20}$ (basically estimated for a year of running). The MINOS sensitivity is also shown.

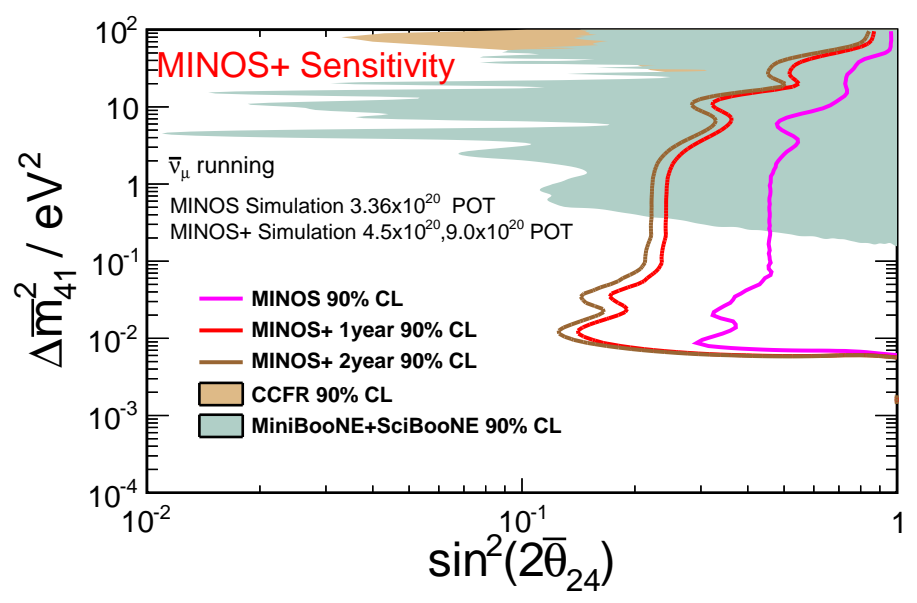

Figure 6.33: The combined MINOS and MINOS+ projected sensitivity for the antineutrino in MINOS+ era for a PoT of $9.0 \times 10^{20}$ (basically estimated for two year of running). The MINOS sensitivity is also shown. 


\subsection{Combination with Bugey in MINOS +}

The combination with Bugey will improve the limit better and this will be interesting because it can be used to compare the LSND and MiNiBooNE signal. The combined MINOS+ Bugey limit is shown in the Fig 6.34

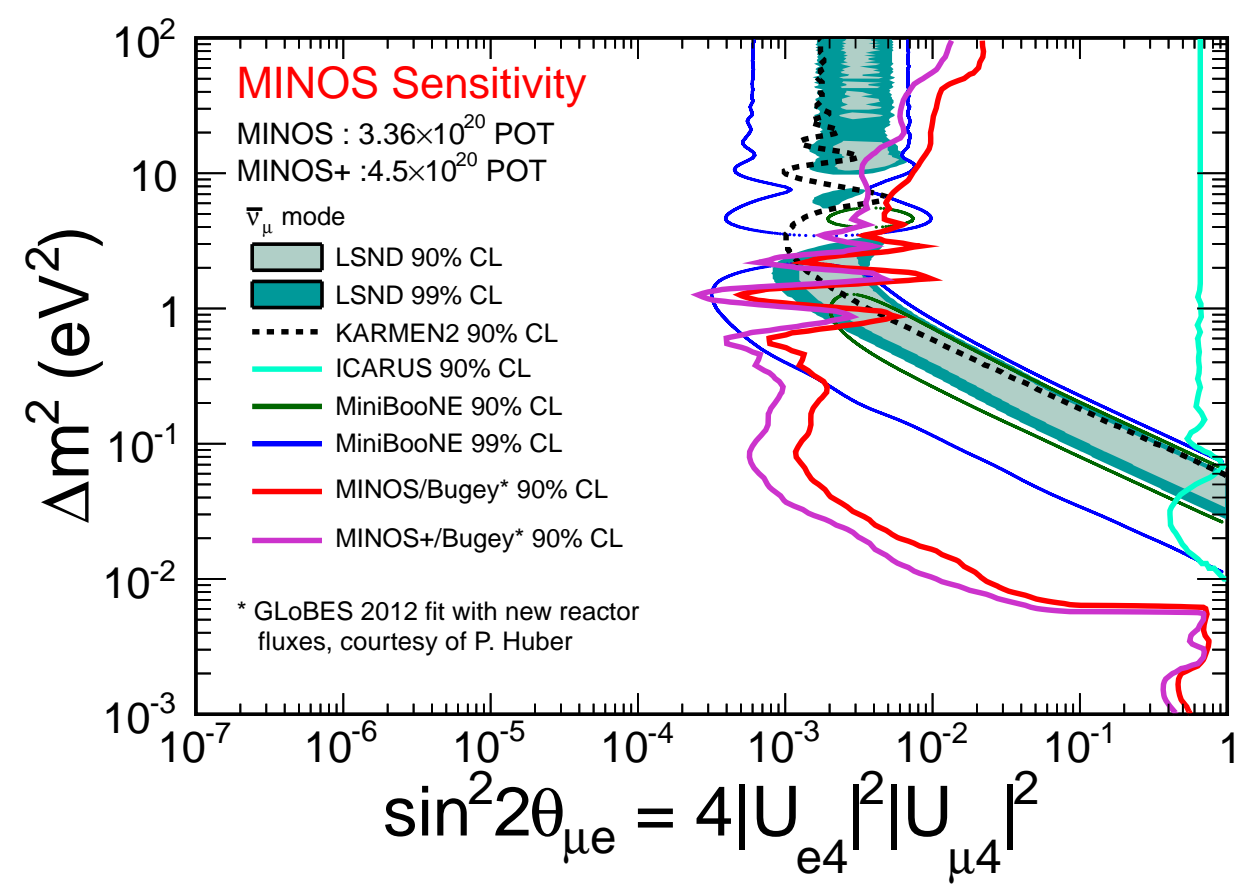

Figure 6.34: The combined Bugey and MINOS+ projected sensitivity for the antineutrino in MINOS+ era for a PoT of $4.5 \times 10^{20}$.

\subsection{Conclusion}

MINOS is capable of putting the limit on the sterile neutrino parameter over a large $\Delta m_{41}^{2}$ range by studying the $v_{\mu} \rightarrow v_{\mu}$ disappearance channel. The sterile sensitivity for the antineutrino mode running is calculated using only the antineutrino CC samples. Much better improvements are possible to this study using the MINOS+ antineutrinos. Combination with other experiments will allow us to compare the appearance signal that LSND and MiNiBooNE found. 



\section{7 \\ Conclusions and Future Scope}

Searching for sterile neutrinos using long baseline neutrino experiment can probe a large range of parameter space that controls the sterile neutrino oscillation. MINOS experiment has looked for the sterile neutrinos using its antineutrino mode data and set a limit on sterile neutrino mixing parameter. A $3+1$ sterile model is used and set limit on $\theta_{24}$ in the range of $10^{-3} \mathrm{eV}^{2}<\Delta m_{41}^{2}<10^{2} \mathrm{eV}^{2}$. Both LSND and MiniBooNE experiment found the evidence for sterile neutrinos in their antineutrino mode of data. A total of $3.36 \times 10^{20}$ POT data is used and only $\bar{v}_{\mu}$ CC events were used. This is the current world's best limit for $\bar{v}_{\mu}$ disappearance sterile search limit below $0.5 \mathrm{eV}^{2}$. Both LSND and MiniBooNE found evidences for sterile neutrinos in the appearance channel, i.e, $\bar{v}_{\mu} \rightarrow \bar{v}_{e}$, this mixing is controlled by the product of angle $\theta_{14}$ and $\theta_{24}$. So in order to present MINOS results into this angle, we used the result from the reactor experiment BUGEY, it is $\bar{v}_{e} \rightarrow \bar{v}_{e}$ experiment, assuming CPT is invariant we combined our MINOS $\bar{v}_{\mu} \rightarrow \bar{v}_{\mu}$ results with BUGEY and presented the combined appearance limit in terms of angle which is probed by LSND and MiNiBooNE.

Our data is statistically limited, we got 226 Far Detector events in the antineutrino mode. MINOS is currently taking data in the $\mathrm{NO} \nu \mathrm{A}$ era, with higher beam intensity. In future MINOS+ will get much improved statistics and which will be improving the current result. We can also take the antineutrinos in the neutrino mode and combine it with the contour to get an improved limit. Also adding the neutral current sample and the combining neutral current and charged current samples will improve the limit further. A selection for choosing such events has been already done and the details are given in Appendix B. The MicroBooNE experiment at Fermilab with Liquid Argon Time Projection Chamber has started 
taking data in early 2015, which will be looking for the anomaly seen by MiniBooNE and LSND with much higher precision, it will be revolutionary and open new doors in physics if it is discovered.

Neutrinos physics is going through an excited time now and entering into the era of precision measurement. Many experiments are designed to measure the mass hierarchy and $\mathrm{CP}$-violation phase $\left(\delta_{\mathrm{cp}}\right)$. Along with these measurements, sterile neutrino searches will be also a focusing area of research in neutrino physics in the coming years. The future neutrino experiments designed at Fermilab using the high intensity neutrino beam will be answering a few of these questions. 


\section{A \\ Beam Matrix Extrapolation}

This section describes the beam matrix extrapolation technique used in MINOS for extrapolating Near Detector flux to Far Detector using the information of parent particle kinematics which produces neutrino. Identical Near and Far Detector design of MINOS is intended to allow the cancellation of the various systematics uncertainty. The uncertainty which affect the both detector in the same way, such as neutrino flux, cross-section, and the modelling of hadronic energy cancel out. The cancellation of these systematics would be exact only if the flux is same in Near and Far Detector. In the MINOS experiment the flux at two detector is not same because of the kinematics of the parent particle that produces neutrinos.

\section{A.1 Extrapolation Method}

For a given parent particle the daughter neutrino energy in the center of mass frame can be calculated. Let $q_{p}$ be the four momentum of the parent particle, $m_{p}$ is the mass of the parent particle and $q_{\mu}$ and $m_{\mu}$ as that of muons and neutrino four momentum is $q_{v}$ and its mass is neglected. The four momentum transfer is given by,

$$
q_{\mu}=q_{p}-q_{v}
$$

Taking the dot product of equation A.1 with itself, we get,

$$
m_{\mu}^{2}=m_{p}^{2}+2\left(E_{v}^{*} m_{p}-0\right) \Rightarrow E_{v}^{*}=\frac{m_{p}^{2}-m_{\mu}^{2}}{2 m_{p}}
$$

From center mass frame it can be transformed into lab frame, moving to the lab frame, if the parent three momentum is $\mathbf{p}_{\mathrm{p}}$ and the parent decay 
point $\mathbf{r}_{\mathrm{v}}$ and $\theta$ is the angle between the parent and neutrino directions of flight is given by.

$$
\cos \theta=\frac{\left(\mathbf{r}_{\mathrm{d}}-\mathbf{r}_{\mathrm{v}}\right) \cdot \mathbf{p}_{\mathrm{p}}}{\left|\mathbf{r}_{\mathrm{d}}-\mathbf{r}_{\mathrm{v}} \| \mathbf{p}_{\mathrm{p}}\right|}
$$

The neutrino energy in the lab frame is (center of mass frame momenta with asterisk)

$$
\begin{aligned}
q_{\mu}^{*} \cdot q_{p}^{*} & =q_{p} \cdot q_{v} \\
& =E_{p}^{*} E_{v, N}^{*}-\mathbf{p}_{\mathrm{p}}^{*} \cdot \mathbf{p}_{v}^{*}=E_{p} E_{v, N}-\mathbf{p}_{\mathrm{p}} \cdot \mathbf{p}_{v} \\
& =m_{p} E_{v, N}^{*}-0=E_{p} E_{v, N}-\left|\mathbf{p}_{\mathrm{p}} \| \mathbf{p}_{v}\right|
\end{aligned}
$$

The energy of neutrino is

$$
\begin{aligned}
E_{v, N} & =\frac{m_{p} E_{\gamma, N}^{*}}{E_{p}-\left|\mathbf{p}_{\mathrm{p}}\right| \cos \theta} \\
& =\frac{E_{\gamma, N}^{*}}{\gamma_{p}\left(1-\beta_{p} \cos \theta\right)}
\end{aligned}
$$

where $\gamma_{p}$ is the parent's Lorentz factor and $\beta_{p}$ is its velocity. It is clear that in the lab frame neutrino energy depends on the relative angle, $\theta$, between the direction of travel of the parent and neutrino. Neutrino flux is also a function of angle. In the parents rest frame neutrinos are emitted isotropically, where as in the lab frame it depends on the angle.

$$
\frac{d N}{d \cos \theta}=\frac{1}{2 \gamma_{p}^{2}\left(1-\beta_{p} \cos \theta\right)^{2}}
$$

MINOS Far Detector is $735 \mathrm{~km}$ away from the source, so only those neutrino which produced at very small range of angles w.r.t the initial beam will reach Far Detector. So we can assume, for the neutrinos which reach at FD will have a unique $\theta$. But for ND covers a wide solid angle as it is close to the decay point. Therefore same parent produce neutrinos at range of energy at Near Detector (Fig B.6). Consequently the neutrino energy spectrum at ND and FD are slightly different (Fig A.2). The effect is more for those parents with higher energy and travel further down the decay before decaying. This enhances the solid angle effect and allows 
wider range of contributing decay angles at ND (this outweighs the increased Lorentz boost whose effect is narrow the outgoing neutrino energy distribution). Thus ND neutrino energy is lower as compared to that of the Far Detector neutrino energy, shifting the neutrino events at the ND downward into the peak. The net effect is that at ND spectrum is more peaked as compared to that of Far Detector.

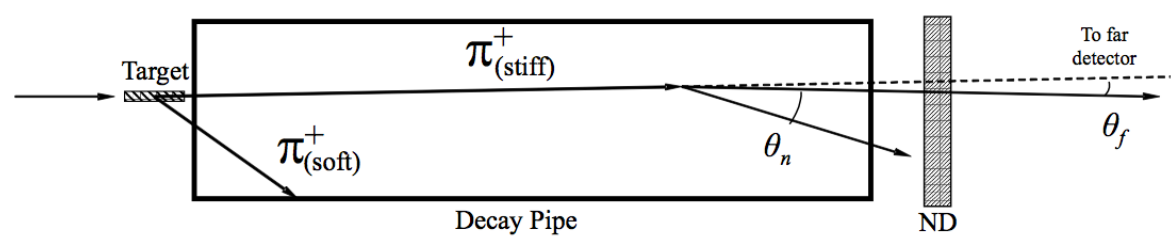

Figure A.1: Schematic representation of neutrino parents in the NuMI decay pipe, illustrating the different solid angles subtended by the Near and Far Detectors at the parent decay point. Diagram taken from [81].
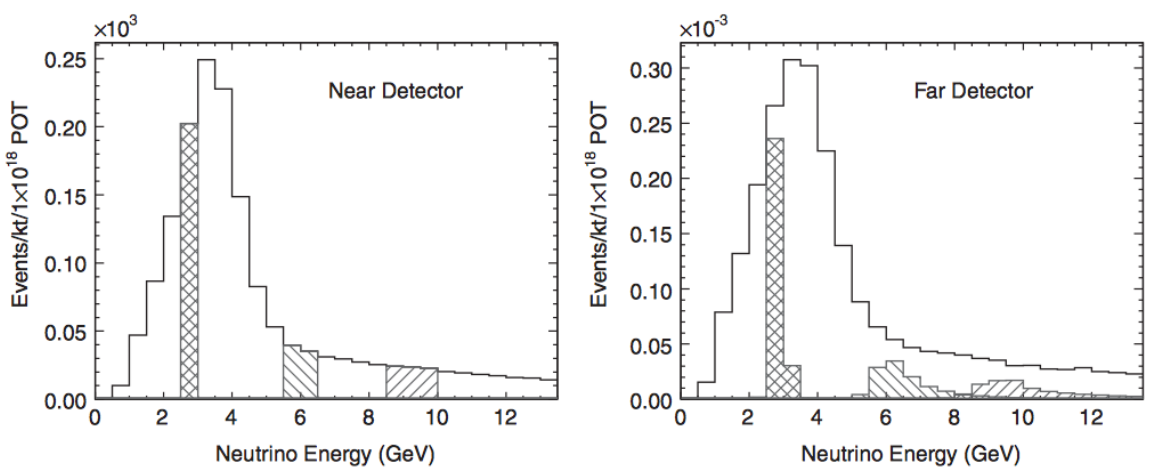

Figure A.2: The connection between the energy of neutrinos observed in ND and neutrinos observed in FD. Decays producing neutrinos with a given energy in the Near Detector would produce a range of energies in the Far Detector, yielding the energy smearing seen here. Figure taken from 81 .

For Predicting the Far detector spectrum given the Near detector neutrino energy spectrum a method is used called "beam matrix". The Monte Carlo is used to produce beam matrix, which connects the Near Detector 
energy spectrum to Far Detector spectrum. The row of the matrix is true Far Detector energy and the column corresponds to the Near detector true energy. This matrix is created by using the decay kinematics information of the parent particle, simulated neutrino parents are allowed to decay in Near and Far Detector at randomly selected interaction vertices. The probabilities of the decay direction and neutrino energy are determined by the kinematics. Then the neutrino energy at ND can be relates with FD via the shared parents. Repeat this procedure for all the simulated parents in order to get the complete matrix. This matrix is calculate for different run period and different mode of running in order to take in account differences in the beam properties, and account for the different parent for neutrinos and antineutrinos. The matrix is shown in Fig A.3.

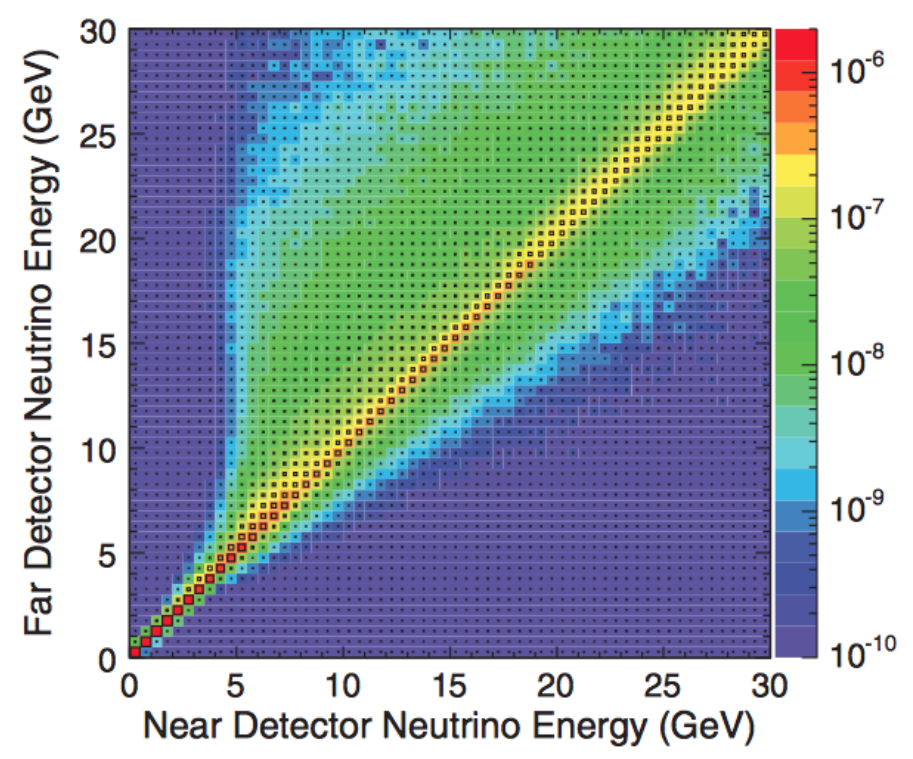

Figure A.3: The joint distribution of neutrino energies observed in the Near and Far Detector. The contents of each cell represent the mean number of $v_{\mu}$ events expected in the Far Detector for one event in the Near Detector. This distribution may be treated as a matrix which relate the energy spectra measured in the Near Detector to those in the Far Detector [81].

The beam matrix is based on the simulation, which assumes the ND 
and FD spectra are in true energy and it is pure. It also assumes the events are selected with $100 \%$ efficiently. In reality there are background contamination and selection is not perfect. Also the beam matrix transform the ND flux to FD flux. But ND and FD do not measure the neutrino flux directly, instead it measures the visible neutrino energy. So additional steps are required to take in account these effects into the extrapolation procedure.

The reconstructed Near Detector is converted into true energy and apply correction for the reconstruction efficiency, selection efficiency and for purity to get the ND flux. This ND flux is then extrapolated to the FD using the beam matrix and the procedure is applied in the reverse order to convert the FD flux into the FD reconstructed energy spectrum. The flowchart for this procedure is shown in Fig A.4.

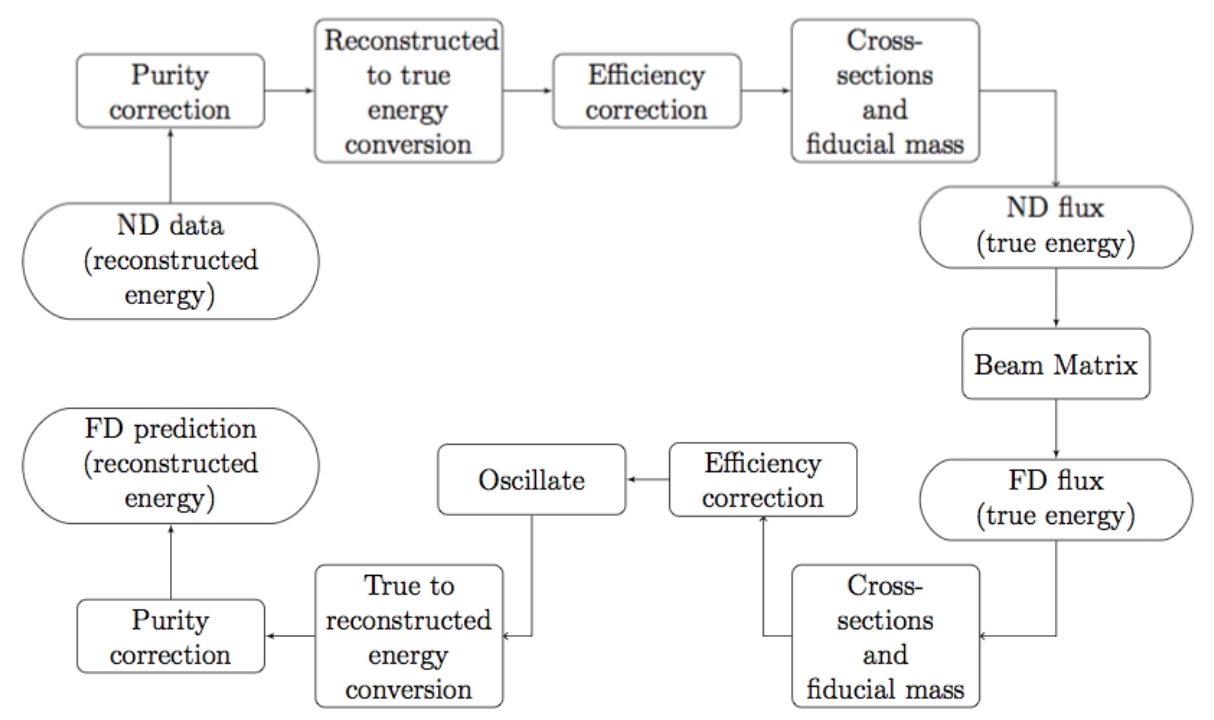

Figure A.4: The procedure for the beam matrix extrapolation to get the FD energy spectrum from the ND reconstructed energy spectrum. Figure taken from [69].

To test this method we can make the FD prediction using the ND Monte Carlo and set the oscillation parameter to zero. If the method is properly working the resulting FD spectrum should exactly match the unoscillated FD Monte Carlo. We would not expect the spectra match 
exactly same because the ND and FD Monte Carlo are statistically independent. This deviation is very small as compare to FD data and can be neglected. MINOS uses the other direct extrapolation techniques also for predicting the Far Detector energy spectrum. The details can be seen in [81]. 


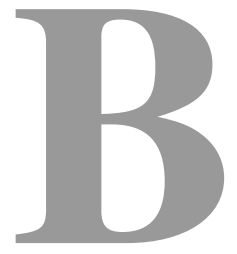

\section{Neutral Current Event Selection at Near Detector for Antineutrino}

\section{B.1 Introduction}

The neutral current cross section are same for all three active neutrinos. Therefore neutral current events are one way to look for sterile neutrinos. Any deviation from the expected number of events at the Far detector could be a signal for sterile neutrinos (number of neutral current events do not change among active neutrinos). So it is very important that to select the neutral current event at the Near Detector in order to predict the Far Detector neutral current event. The high event rate at the Near Detector makes the NC selection more difficult. Due to the high event rate and presence of poorly reconstructed events, which are reconstructed as low energy showers makes the neutral current event selection at Near Detector difficult. This problem is unique to Near Detector and not for Far Detector, which makes far-over-near differences. Due to the presence of these poorly reconstructed event the Data and $\mathrm{MC}$ agreement at the lower energy range is very poor for NC selected events. In order to remove this badly reconstructed events two preselection variable were introduced which removes most of this events and make Data-MC agreement better. The reconstruction failure is classified into main three category, split events, leakage events and incomplete events [121. Monte Carlo studies shows that for the split events and incomplete events the eventCompleteness is less than 0.5 (eventCompleteness $<0.5$ ). The event completeness is defined as the ratio of the reconstructed pulse-height in the event truly attributed to that specific neutrino interaction divided by the the total reconstructed pulse height that the neutrino deposited in 
Neutral Current Event Selection at Near Detector for

the detector. However the event completeness gives no information about the event migrating from outside of the detector to inside and vice-versa. To incorporate all these events as background a new definition is assigned for these events, these are called poorly reconstructed events. The poorly reconstructed events are characterised by a low value of the ratio of the reconstructed shower energy divided by the true shower energy. The events are chosen to be poorly reconstructed if,

$$
\frac{\text { reconstructed shower energy }}{\text { true shower energy }}<0.3
$$

which means the ratio of reconstructed shower energy to true shower energy should be less than $30 \%$.

Two variables were introduced to remove these poorly reconstructed events from the samples and remove the background. For neutral current events when shower develops longitudinally it will deposit energy in successive planes. The poorly reconstructed events caused by the reconstruction failures will have small number of maximum contiguous plane. After applying the NC fiducial volume requirements, cut all the events which are having maximum consecutive plane $<3$. Fig B.1(a) shows the Data-MC comparison of the maximum consecutive plane variable, it can be seen that in the bins where the contribution from poorly reconstructed events are higher the Data-MC agreement is poor.

The other pre-selection variable is slice pulse height fraction, which is defined as, the event pulse height divided by the slice (collection of hits which are close in time and space) pulse height. The events are removed if the slice pulse height fraction is $<0.5$. Those events which will have extra activity which are not part of the events (basically due to split event, incomplete events, and leakage events) are removed when applying this cut. Fig B.1(b) shows the Data-MC comparison of the slice pulse height fraction variable with the poorly reconstructed component. 


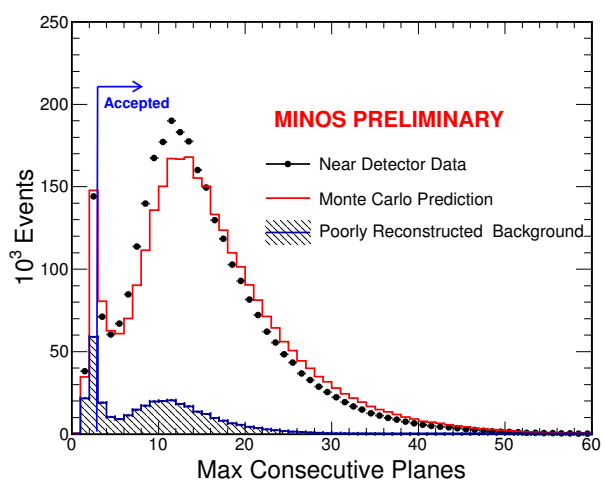

(a)

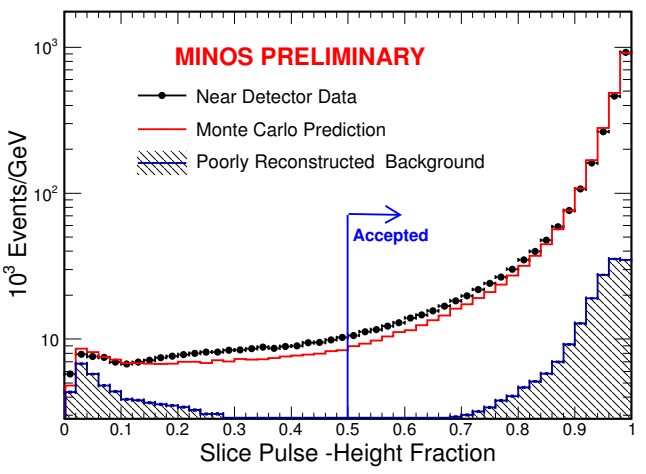

(b)

Figure B.1: The Near Detector pre-selection variables a) shows the DataMC comparison of the maximum consecutive plane variable, the contribution from the poorly reconstructed events are also show b) shows the Data-MC comparison of the slice pulse height fraction, the poorly reconstructed component of the events are also shown.

For the energy range $<1 \mathrm{GeV}$ (where most of the shower reconstruction problem arises) the background from poorly reconstructed events are reduced to $11.5 \%$ from $37.5 \%$ after applying the preselection cuts.

These preselection variables are unique to Near Detector not for Far Detector. Therefore it causes a uncertainty in the Far Detector. The systematics error due to these cleaning cuts are evaluated for RHC combined runs between $0-2 \mathrm{GeV}$ energy [121]. Fig B.2 Shows the $\pm 1 \sigma$ error band 
Neutral Current Event Selection at Near Detector for

for the combined RHC runs, it is found the uncertainties in for first 4 bins are $0-0.5 \mathrm{GeV}=12.1 \%, 0.5-1 \mathrm{GeV}=9.7 \%, 1-2 \mathrm{GeV}=6.9 \%, 4.7 \%$

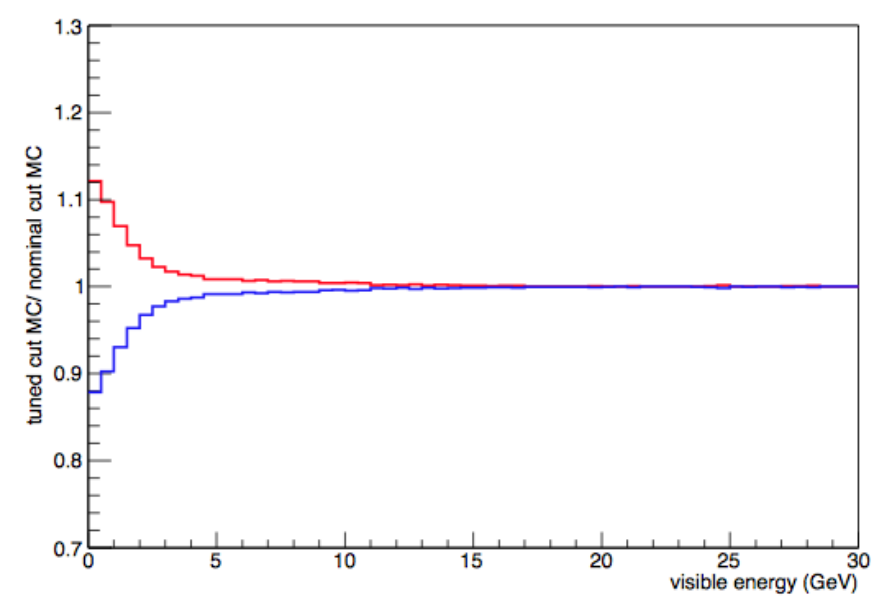

Figure B.2: The error band for the ND preselection cleaning systematic is shown. The values of the systematic errors assign on the first $2 \mathrm{GeV}$ bins are: $12.1 \%, 9.7 \%, 6.9 \%$ and $4.7 \%$. The red line corresponds to the $+1 \sigma$, the blue line to the $-1 \sigma$. The error band is symmetric by constructions.

\section{B.2 Neutral Current Event Selection}

After applying the pre-selection cuts, next procedure is to distinguish the Neutral Current events and charged current events. The goal of event selection is to maximise the efficiency and purity of the selected neutral current sample. Using Monte Carlo event samples, efficiency is defined as the number of true events selected, divided by the total number of true events after passing the data-quality criteria. Purity is defined as the ratio of the number of true selected events to the sum of total number of events selected and background. The variable for selecting the neutral current events are based on reconstruction variable which shows clear difference from charged current event. The classification variables using for the neutral current events are event length and track extension. Event length is expressed as the difference between the first and last active plane in the event and track extension is defined as the difference between track length and shower length. 
The neutral current samples are obtained after applying specified cut on the above mentioned two classification variable. Neutral current events are shorter than the charged current events, so any events which passes less than 47 planes for which no track is reconstructed are classified as neutral current event. Because neutral current events are short or contains no reconstructed tracks, events crossing fewer than 47 planes that contain a track are classified as neutral current if the track extends fewer than 6 planes beyond the shower (Track Extension). Fig B.3 is showing the Data-MC comparison of this classification variables, the CC background events are also shown.

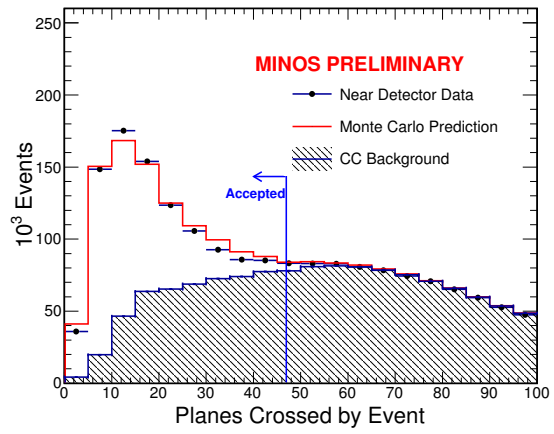

(a)

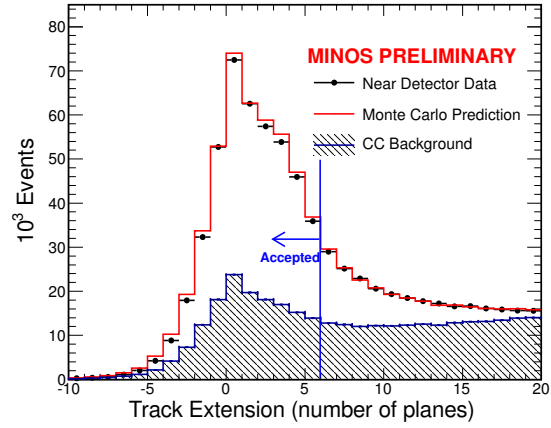

(b)

Figure B.3: Data-MC Comparisons of the neutral current classification variable a) Event length and b) Track Extension. Data and MC shows good agreement, the CC background events are also shown.

Distributions of the vertex positions of NC-selected events are displayed in Fig B.4(c). The plots are area normalised. The plots show good Data/MC agreement for vertex distributions. 
Neutral Current Event Selection at Near Detector for

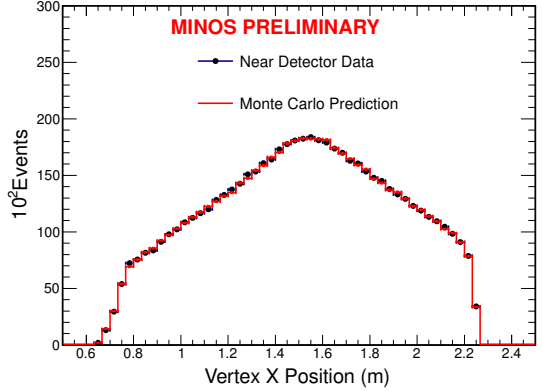

(a)

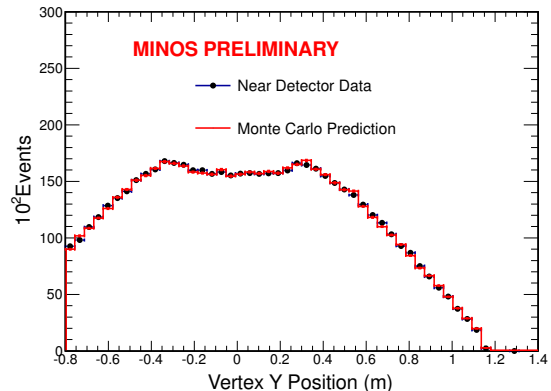

(b)

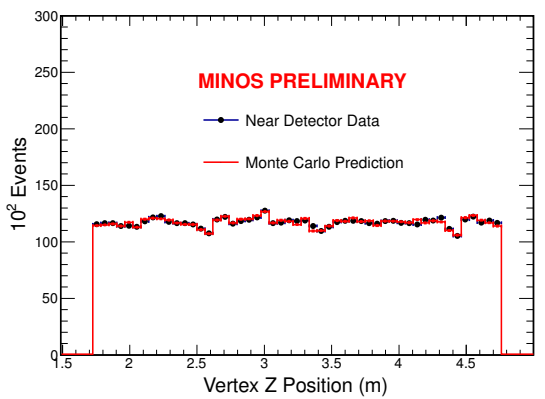

(c)

Figure B.4: Data and MC distributions and ratios for the vertex positions of NC selected events. Fiducial and cleaning cuts are applied along with NC selection cuts. a) Vertex X b) Vertex Y and c) Vertex Z.

Efficiency and Purity for the neutral current selection at Near Detector is shown in Fig B.5(a), The selection has $80 \%$ efficiency and $75 \%$ purity. Definition of efficiency and purity is given by,

$$
\begin{gathered}
\text { Efficiency }=\frac{\text { number of selected true signal events }}{\text { Total number of events before selection }} \\
\text { Purity }=\frac{\text { number of selected true signal events }}{\text { total number of events before selection }+ \text { background in the selected sample }}
\end{gathered}
$$

The selected neutral current energy spectra is displayed on Fig B.5(b) Data-MC comparison shows good agreement. The lower energy discrepancies are due to the presence of poorly reconstructed events. 


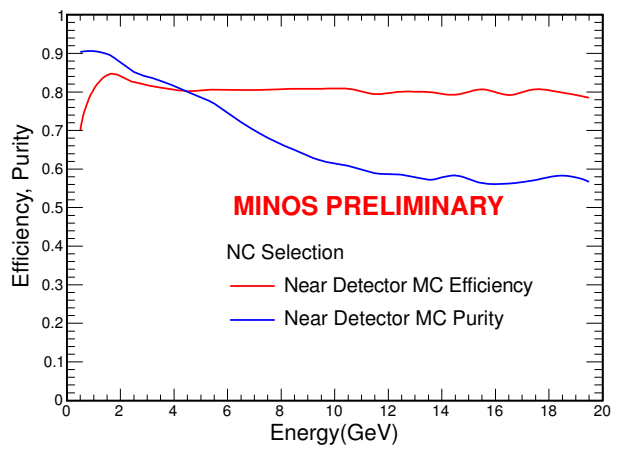

(a)

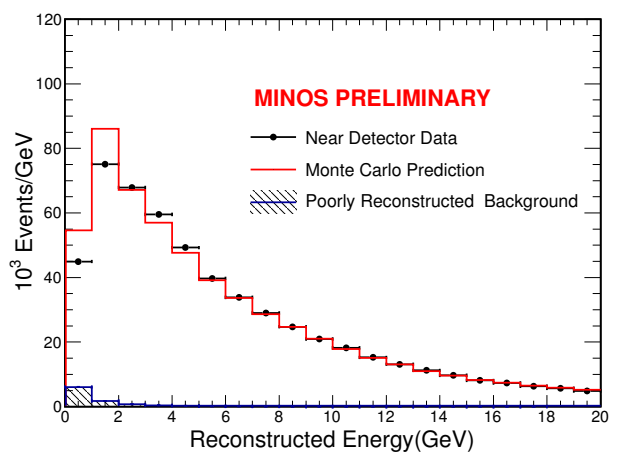

(b)

Figure B.5: a) The efficiency (red) and purity (blue) for the neutral current selection as a function of energy. b) Data-MC comparison of the selected ND neutral current energy spectrum. The bad data-MC agreement in the first two energy bin are due to the presence of the poorly reconstructed events.

\section{B.3 Sterile Neutrino Search Strategy Using NC Events}

Using Monte-Carlo the total neutral current energy spectrum split up into different components. The Fig. B.6 Shows the contribution from different component to the total energy spectrum. The number of NC events is compared using a $R$-Statistic method mentioned in the section. 2.3. Because of the low background from the $\mathrm{CC}$ sample below $5 \mathrm{GeV}$, this 
Neutral Current Event Selection at Near Detector for 156

Antineutrino

$R$-value can be measured with less uncertainty. The $R$ value is given by Equation 2.8. Since rate of neutral current event are independent of oscillation any deviation from unity in the R-value will be a signature for sterile neutrinos.

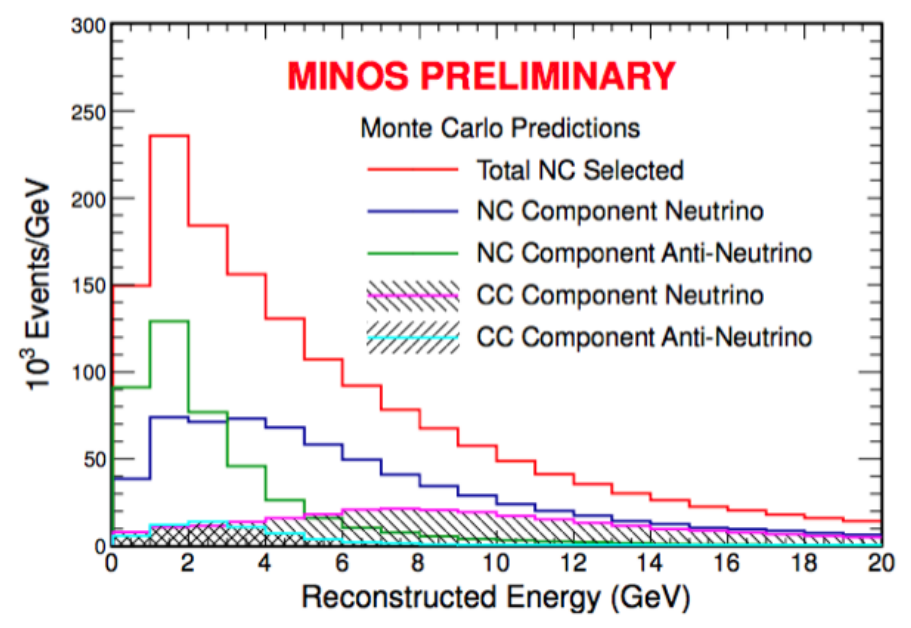

Figure B.6: The total neutral current energy spectra are split up into different component. The $\mathrm{CC}$ background is also shown. Because of the low background below $5 \mathrm{GeV}$, it gives better sensitivity for the sterile neutrino search. 


\section{References}

[1] O. von Baeyer, O. Hahn, and L. Meitner, "Nachweis von $\beta$ - Strahlen bei Radium D," Physikalische Zeitschrift, vol. 12, pp. 378, (1911).

[2] Wolfgang Pauli, "Letter to the physical society of Tubingen", (1930).

[3] F. L. Wilson, "Fermi's Theory of Beta Decay", American Journal of Physics 36, 1150 (1968).

[4] R. P. Feynman and M. Gell-Mann, "Theory of the Fermi Interaction", Phys. Rev. 109, 193 (1958).

[5] E. C. G. Sudarshan and R. E. Marshak, "Chirality Invariance and the Universal Fermi Interaction", Phys. Rev. 109, 1860 (1958).

[6] E. D. Commins and P. H. Bucksbaum, "Weak Interactions of Leptons and Quarks" (Cambridge University Press, 1983).

[7] W. Heisenberg, "Zur Theorie der Schauer in der Ho henstrahlung, Zeitschrift fur Physik" 101, 533 (1936).

[8] S. L. Glashow, "Partial Symmetries of Weak Interactions", Nucl. Phys. 22, 579 (1961).

[9] A. Salam and J. C. Ward, "Electromagnetic and weak interactions", Phys. Lett. 13, 168 (1964).

[10] S. Weinberg, "A Model of Leptons", Phys. Rev. Lett. 19, 1264 (1967).

[11] F. J. Hasert et al., "Search for elastic muon neutrino electron scattering", Phys. Lett. B46, 121 (1973).

[12] F. J. Hasert et al., "Observation of neutrino-like interactions without muon or electron in the Gargamelle neutrino experiment", Phys. Lett. B46, 138 (1973).

[13] G. Arnison et al., "Experimental Observation of Isolated Large Transverse Energy Electrons With Associated Missing Energy at $\sqrt{5} 40 \mathrm{GeV} "$, Phys. Lett. B122, 103 (1983). 
[14] R. Banner et al., "Observation of Single Isolated Electrons of High Transverse Momentum in Events with Missing Transverse Energy at the CERN pp Collider", Phys. Lett. B122, 476 (1983).

[15] G. Arnison et al., "Experimental observation of lepton pairs of invariant mass around $95 \mathrm{GeV} / \mathrm{c}^{2}$ at the CERN SPS collider", Phys. Lett. B126, 398 (1983).

[16] P. Bagnaia et al., "Evidence for $Z^{0} \rightarrow e^{+}+e^{-}$at the CERN anti p-p collider", Phys. Lett. B129, 130 (1983).

[17] C. Cowan, F. Reines, F. Harrison, H. Kruse, and A. McGuire, "Detection of the free neutrino: A Confirmation", Science, vol. 124, pp. 103-104, (1956).

[18] G. Danby, J. Gaillard, K. A. Goulianos, L. Lederman, N. B. Mistry, et al., "Observation of high-energy neutrino reactions and the existence of two kinds of neutrinos",Phys.Rev.Lett., vol. 9, pp. 36-44, (1962).

[19] K. Kodama et al., "Observation of tau neutrino interactions",Phys.Lett., vol. B504, pp. 218-224, (2001).

[20] J. Beringer et al., "Review of particle physics (rpp)", Phys.Rev., vol. D86, p. 010001, (2012).

[21] S. Schael et al., "Precision electroweak measurements on the $\mathrm{Z}^{0}$ resonance", Phys.Rept., vol. 427, pp. 257-454, (2006).

[22] P. Ade et al., "Planck 2013 results. xvi. cosmological parameters", arXiv, (2013).

[23] Son V Cao ,Ph.D Thesis, The University of Texas at Austin(2014).

[24] B. Pontecorvo, "Inverse beta processes and non-conservation of lepton charge", Sov. Phys. JETP 7, 172 (1958).

[25] Z. Maki, M. Nakagawa, and S. Sakata, "Remarks on the unified model of elementary particles", Prog. Theor. Phys. 28, 870 (1962). 
[26] D. M. Webber et al., "An improved measurement of electron antineutrino disappearance at Daya Bay",Nucl.Phys.Proc.Suppl., vol. 233, pp. 96- 101, (2012).

[27] J. Ahn et al., "Observation of reactor electron antineutrino disappearance in the RENO experiment", Phys.Rev.Lett., vol. 108, p. 191802, (2012).

[28] Y. Abe et al., "Indication for the disappearance of reactor electron antineutrinos in the Double Chooz experiment", Phys.Rev.Lett., vol. 108, p. 131801, (2012).

[29] R.Patterson et al., "The $\mathrm{NO} \nu \mathrm{A}$ experiment:Status and outlook",Nucl.Phys.Proc.Suppl.,vol. 235-236, pp.151-157, (2013).

[30] K. Abe et al., "Observation of electron neutrino appearance in a muon neutrino beam", Phys.Rev.Lett., vol. 112, p. 061802, (2014).

[31] P. Adamson et al, "Combined Analysis of $\nu_{\mu}$ Disappearance and $\nu_{\mu} \rightarrow \nu_{e}$ Appearance in MINOS Using Accelerator and Atmospheric Neutrino".Phys. Rev. Lett. 112, 191801, (2014).

[32] John N. Bahcall, Aldo M. Serenelli, and Sarbani Basu, "New solar opacities, abundances, helioseismology, and neutrino fluxes",Astrophys. J., vol. 621, L85-L88, (2005).

[33] K. Hirata et. al., "Observation of B-8 Solar Neutrinos in the Kamiokande-II Detector", Phys. Rev. Lett., vol. 63, p. 16, (1989).

[34] D. Abdurashitov, V. Gavrin, S. Girin, V. Gorbachev, T. V. Ibragimova, et al., The Russian-American gallium experiment (SAGE) Cr neutrino source measurement, Phys.Rev.Lett., vol. 77, pp. 47084711, (1996).

[35] P. Anselmann et al., First results from the Cr-51 neutrino source experiment with the GALLEX detector, Phys.Lett., vol. B342, pp. 440-450, (1995).

[36] J. Boger et. al., "The Sudbury neutrino observatory", Nucl. Instrum. Meth. A, vol. 449, pp. 172-207, (2000). 
[37] P. Langacker et. al., "Implications of the Mikheyev-SmirnovWolfenstein (MSW) mechanism of amplification of neutrino oscillations in matter", Nuc. Phys. B, vol. 282, pp. 589-609, (1987).

[38] J. Beringer et. al. (Particle Data Group), Phys. Rev. D vol. 86, pp. 010001, (2012).

[39] S. Abe et. al., "Precision Measurement of Neutrino Oscillation Parameters with KamLAND", Phys. Rev. Lett., vol. 100, pp. 221803, (2008).

[40] G. L. Fogli, E. Lisi, A. Marrone, D. Montanino, A. Palazzo, et. al., "Global analysis of neutrino masses, mixings and phases: entering the era of leptonic CP violation searches" Phys. Rev. D, vol. 86, pp. 013012, (2012).

[41] K. Hirata et. al., "Experimental study of the atmospheric neutrino flux", Physics Letters B, vol. 205, no. 2-3, pp. 416-420, (1988).

[42] Y. Fukuda et al. "Evidence for oscillation of atmospheric neutrinos". Phys. Rev. Lett., 81(8):1562-1567, (1998).

[43] P. Adamson et. al., "Measurement of Neutrino and Antineutrino Oscillations Using Beam and Atmospheric Data in MINOS", vol. 110, pp. 251801, (2013).

[44] M. Apollonio et. al., "Search for neutrino oscillations on a long baseline at the CHOOZ nuclear power station", Eur. Phys. J. C, vol. 27, pp. 331-374, (2003).

[45] F. Boehm et. al., "Final Results from Palo Verde neutrino oscillation experiment",Phys. Rev. D, vol. 64, pp. 112001, (2001).

[46] Y. Abe et. al., "Reactor $\nu_{e}$ disappearance in the Double Chooz experiment", Phys. Rev. D, vol. 86, pp. 052008, (2012).

[47] F. P. An et. al., "Improved Measurement of Electron Antineutrino Disappearance at Daya Bay.", Chin. Phys. C, vol. 37, pp. 011001, (2013). 
[48] J. K. Ahn et. al., "Observation of Reactor Electron Antineutrinos Disappearance in the RENO Experiment", Phys. Rev. Lett., vol. 108, pp. 191802, (2012).

[49] K. Abe et. al., "Evidence of electron neutrino appearance in a muon neutrino beam", Phys. Rev. D, vol. 88, pp. 032002, (2013).

[50] P. Adamson et al. "Electron Neutrino and Antineutrino Appearance in the Full MINOS Data Sample" Phys. Rev. Lett. 110, 171801.

[51] Hisakazu Minakata, "Phenomenology of future neutrino experiments with large Theta(13)",arXiv:1209.1690 [hep-ph] .

[52] Corey Adams et.al.,"The Long-Baseline Neutrino Experiment: Exploring Fundamental Symmetries of the Universe" arXiv:1307.7335 [hep-ex].

[53] Fengpeng An et.al., "Neutrino Physics with JUNO." arXiv:1507.05613 [physics.ins-det].

[54] Daljeet Kaur et.al., "The sensitivity of the ICAL detector at Indiabased Neutrino Observatory to neutrino oscillation parameters" arXiv:1409.2231 [hep-ex] .

[55] T. Patzak et.al., J. Phys.: Conf. Ser. 375, 042056 (2012).

[56] K. Abe et al., "Letter of Intent: The Hyper-Kamiokande Experiment - Detector Design and Physics Potential" , arXiv:1109.3262 [hep-ex].

[57] P. Adamson et. al., "CHerenkov detectors In mine PitS (CHIPS) Letter of Intent to FNAL"arXiv:1307.5918 [physics.ins-det].

[58] A. Aguilar-Arevalo et al. [LSND Collaboration], Phys. Rev. D 64, 112007 (2001).

[59] (ALEPH Collaboration), "Precision Electroweak Measurements on the Z Resonance". arXiv:hep-ex/0509008.

[60] S. Pascoli et.al.,"Leptogenesis and Low Energy CP Violation in Neutrino Physics." arXiv:hep-ph/0611338. 
[61] A. Aguilar et al., "Evidence for neutrino oscillations from the observation of $\bar{\nu}_{e}$ appearance in a $\bar{\nu}_{\mu}$ beam", Phys. Rev. D 64 (2001) 112007, [hep-ex/0104049].

[62] A. Aguilar-Arevalo et al., Event Excess in the MiniBooNE Search for $\bar{\nu}_{\mu} \rightarrow \bar{\nu}_{e}$ Oscillations, Phys.Rev.Lett., vol. 105, p. 181801, (2010).

[63] G. Mention, M. Fechner, T. Lasserre, T. Mueller, D. Lhuillier, et al., The Reactor Antineutrino Anomaly, Phys.Rev., vol. D83, p. 073006, (2011).

[64] K. N. Abazajian et al., arXiv:1204.5379.

[65] A. Aguilar-Arevalo et al. (MiniBooNE Collaboration), Phys.Rev.Lett. 102, 101802 (2009), arXiv:0812.2243 [hep-ex].

[66] A. A. Aguilar-Arevalo et al. (MiniBooNE Collaboration), Phys.Rev.Lett. 103, 061802 (2009), arXiv:0903.2465 [hep-ex].

[67] T. Mueller, D. Lhuillier, M. Fallot, A. Letourneau, S. Cormon, et al., Improved Predictions of Reactor Antineutrino Spectra, Phys.Rev., vol. C83, p. 054615, 2011. 1996.

[68] J. Kopp, P.A.N. Machado, M. Maltoni and T. Schwetz, Sterile neutrino oscillations: the global picture, JHEP 05 (2013) 050 [arXiv:1303.3011]

[69] J. J. Evans, "Measuring Antineutrino Oscillations with the MINOS Experiment". PhD thesis, University of Oxford, (2008).

[70] P. Adamson et al, "Active to Sterile Neutrino Mixing Limits from Neutral-Current Interactions in MINOS". Phys.Lett. 107, 011802 (2011).

[71] P. Adamson et. al "Search for sterile neutrino mixing in the MINOS long-baseline experiment". Phys. Rev. D 81, 05 (2004).

[72] P. Adamson et al. "Electron Neutrino and Antineutrino Appearance in the Full MINOS Data Sample", Phys. Rev. Lett. 110, 171801.

[73] P. Adamson et al, "Search for flavor-changing non-standard neutrino interactions by MINOS", Phys. Rev.D 88, 07(2011). 
[74] P. Adamson et al. "Precision measurement of the speed of propagation of neutrinos using the MINOS detectors" , arXiv:1507.04328.

[75] Justin Evans, "The MINOS experiment: results and prospects".arXiv:1307.0721 [hep-ex] .

[76] D. Michael et. al., "The Magnetised steel and scintillator calorimeters of the MINOS experiment", Nucl.Instrum.Meth., vol. A596, pp. 190-228, (2008).

[77] A.Belias, etal., IEEE Trans. Nucl. Sci. NS-51, 451, (2004).

[78] P. Ochoa, Search for $\nu_{e}$ appearance with the LEM selection. PhD thesis, Caltech, (2009).

[79] G. Tzanankos et al., "MINOS+: a proposal to fnal to run MINOS with the medium energy NuMI beam", FERMILAB-PROPOSAL1016, 2011. MINOS docdb 7923.

[80] J.S. Marshall, Ph.D thesis, University of Cambridge, (2008).

[81] P. Adamson et al." Study of muon neutrino disappearance using the Fermilab Main Injector neutrino beam", Phys. Rev. D 77, 072002

[82] P. Ballester, "Hough transform for robust regression and automated detection", Astron.Astrophys., vol. 286, pp. 1011, 1994.

[83] A. Strandlie and R. Fruhwirth, "Track and vertex reconstruction: From classical to adaptive methods", Rev.Mod.Phys. 82, 1419 (2010).

[84] R. Fruhwirth,"Application of Kalman filtering to track and vertex fitting”, Nucl.Instrum.Meth.A262, 444 (1987).

[85] P. Billoir and S. Qian, "Fast vertex fitting with a local parametrization of tracks", Nucl.Instrum.Meth. A294, 219 (1990).

[86] A. Cervera-Villanueva et al., "Kalman filter tracking and vertexing in a silicon detector for neutrino physics", Nucl.Instrum.Meth. A486, 639 (2002). 
[87] R. Brun et. al. Geant detector description and simulation tool. CERN Program Library Long Writeup W5013, (1994).

[88] C. J. Backhouse, "Measuring neutrino oscillation parameters using $\nu_{\mu}$ disappearance in MINOS", Ph.D. thesis, (2011).

[89] M. Campanella, A. Ferrari, P. Sala, and S. Vanini, "First Calorimeter Simulation with the FLUGG Prototype", ATL-SOFT-99-004, (1999).

[90] M. Campanella, A. Ferrari, P. Sala, and S. Vanini, "Reusing Code from FLUKA and GEANT4 Geometry," ATL-SOFT-98-039, 13 October (1998).

[91] G. Battistoni, F. Cerutti, A. Fasso, A. Ferrari, S. Muraro, J. Ranft, S. Roesler, and P. R. Sala, "The FLUKA code: description and benchmarking",AIP Conf. Proc., vol. 896, pp. 31-49, (2007).

[92] R. Brun, F. Carminati, and S. Giani, "GEANT Detector Description and Simulation Tool", CERN-W5013, (1994).

[93] A. Fasso, A. Ferrari, S. Roesler, P. Sala, F. Ballarini, et al., "The physics models of FLUKA: Status and recent developments", e Conf, vol. C0303241, (2003).

[94] A. Bodek and J. L. Ritchie. "Fermi motion effects in deep inelastic lepton scattering from nuclear targets".Phys. Rev., D23:1070, (1981).

[95] C. H. Llewellyn Smith. "Neutrino reactions at accelerator energies". Phys. Rept., 3:261, 1972.

[96] Dieter Rein and Lalit M. Sehgal. "Neutrino excitation of baryon resonances and single pion production".Ann. Phys., 133:79, (1981).

[97] Dieter Rein and Lalit M. Sehgal." Coherent pi0 production in neutrino reactions". Nucl. Phys., B223:29, (1983).

[98] T. Yang et. al., "A hadronization model for few-GeV neutrino interactions", arXiv:hep- ph/0904.4043, (2009). 
[99] T. Sjostrand, S. Mrenna, and P. Skands, "PYTHIA 6.4 physics and manual", Journal of High Energy Physics, vol. 05, pp. 026, (2006).

[100] Z. Koba, H. B. Nielsen, and P. Olesen, "Scaling of multiplicity distributions in high- energy hadron collisions",Nucl. Phys. B, vol. 40, pp. 317-334, (1972).

[101] R. Merenyi et. al., "Determination of pion intranuclear rescattering rates in $\nu_{\mu}$ - Ne versus $\nu_{\mu}-\mathrm{D}$ interactions for the atmospheric $\nu$ flux",Phys. Rev. D, vol. 45(3), pp. 743-751, (1992).

[102] M. A. Kordosky, "Hadronic Interactions in the MINOS Detectors", PhD thesis University of Texas at Austin, (2004).

[103] A. Clark, R. Field, H. Frisch, W. Holley, R. Johnson, L. Kerth, R. Sah, and W. Wenzel, Observed difference in the ranges of positive and negative muons, Physics Letters B 41 (1972), no. 2, 229 -233.

[104] Jeff J Hartnell, Measurement of the Calorimetric Energy Scale in MINOS.

[105] A. Clark, R. Field, H. Frisch, W. Holley, R. Johnson, L. Kerth, R. Sah, and W. Wenzel, "Observed difference in the ranges of positive and negative muons", Physics Letters B 41 (1972), no. 2, 229 - 233.

[106] "Position Paper on the Fitting Procedure for the 2013 Sterile Analysis". Alena V.Devan MINOS DocDB-9817.

[107] Joao Coehlo et. al "Blessing Package for the 2014 Sterile Analysis". MINOS DocDB-10583.

[108] Alexandre Radovic "A Position Paper Describing a Hadron Production Uncertainty Covariance Matrix" . MINOS DocDB - 10569.

[109] Alexandre Radovic, "Hadron Production Uncertainties for RHC Sterile". MINOS DocDB 10908.

[110] Adam Aurisano "A Position Paper on Constructing Systematic Covariance Matrices for the 2014 Sterile Analysis" . MINOS DocDB $-10581$. 
[111] MINOS RHC Sterile Updates. Navaneeth Poonthottathil MINOS DocDB 10745.

[112] Ashley Timmons "Beam Optic Systematic Matrices for the 2014 Sterile Analysis". MINOS DocDB -10580.

[113] RHC Sterile Updates. Navaneeth Poonthottathil MINOS DocDB 10804.

[114] G.J Feldman and R.D Cousins, Phys.Rev.D 57,3873 (1988).

[115] B. Rebel, "General Form of the Unitary Mixing Matrix for 4 Neutrino Mass Eigen-states", MINOS-doc-4995 (2009).

[116] Y. Fukuda et. al., (Super-Kamiokande Collab.), Phys. Rev. Lett., vol. 81, pp. 1562, (1998).

[117] P. L. Vahle, Electromagnetic interactions in the MINOS detectors, Ph.D. thesis, University of Texas at Austin, Fermilab-Thesis-200435 (2004).

[118] C. Zeitnitz and T. Gabriel, "The GEANT - CALOR interface and benchmark calculations of ZEUS test calorimeters", Nucl.Instrum.Meth., vol. A349, pp. 106-111, (1994).

[119] Richa Sharma, Ph.D Thesis, Punjab University (2015).

[120] Y. Fukuda et. al., (Super-Kamiokande Collab.), Phys. Rev. Lett., vol. 81, pp. 1562,1998.

[121] Gemma Maria Tinti, Ph.D Thesis, The University of Oxford (2010). 